\title{
Efecto del uso de fertilizantes y pesticidas sobre organismos acuáticos en arroyos de la zona hortícola de La Plata
}

\author{
Lic. Marina Arias
}

Directores

Dr. Carlos Bonetto

Dr. Hernán Mugni

Facultad de Ciencias Naturales y Museo

Universidad Nacional de La Plata

Año 2019 


\section{AGRADECIMIENTOS}

A mis Directores de tesis, Carlos y Hernán, por aceptarme sin conocerme y dirigir este trabajo. A Carlos por bancar todas mis ideas y a Hernán por ayudarme a ponerlas en práctica. A ambos por la paciencia y el incentivo.

A las jurados Carolina Ocón, Karina Milgioranza y Alejandra Rumi por la gran valoración de esta tesis y por las sugerencias y correcciones que enriquecieron este trabajo.

A mis compañerxs de laboratorio: a Nati y Marina, de quienes aprendí cuando me incorporé; a Silvia por su disposición y su ayuda permanente; a Any, por las campañas compartidas y por segundearme en todas; a Ariel, su contribución, desde los muestreos hasta el análisis de los datos, fue fundamental para el desarrollo de este trabajo, y también por la buena onda y la amistad. $Y$ a Nacho, el mejor compañero que podría haber elegido.

A Noe Ferrando, por su invaluable colaboración en esta tesis y sobre todo, por haber compartido sus conocimientos de manera desinteresada, por su paciencia y compañerismo. A Hernán Benítez por su ayuda con los cultivos, en el laboratorio y en los muestreos. A Roberto Jensen por todo el laburo en el campo. A la banda de Peces por el aguante, el agite y el espacio. A Ixs Becarixs ILPLA por su compañerismo y organización, un orgullo formar parte de este grupo. A Estefy Paz, Joaquín Cochero, Analía Díaz y a todo el ILPLA; todxs colaboraron de alguna u otra manera en la realización de este trabajo.

A Juan Cesar Paggi y a Laura Miserendino por sus conocimientos y experiencia, al grupo de Insectos Acuáticos de la Universidad de Buenos Aires y al Laboratorio de Cromatografía de la Universidad Nacional de Comahue. A la Cátedra de Ecología General de la FCNyM, en especial a Ana Salazar y a Maria Elicia Mc Donagh. A Guido Borzi por las dudas hidrológicas, a Santi Torres y Mary Derguy por las dudas geográficas. A Caro Baldini por el territorio y por su amistad.

A la banda del árbol por los almuerzos descontracturantes, en especial al grupo de Parásitos Acuáticos del CEPAVE. A mis amigas Sofía y Mariana, con quienes empecé esta aventura, y a Eliana, Eugenia, Julia y Mercedes; tenerlas cerca fue lo mejor de lo cotidiano. También a Noelia y Luisina que estuvieron siempre y a Mariana Chuliver, por estar a pesar de los kilómetros.

A la Colonia (Leti, Mar, Carlos, Cuba, Lucre, Nela, Natila, Cat, Pepi y toda la manada), por ser mil segundo hogar, la familia y el aguante, por el fuego, el brillo, la 
mantita y la aventura. También a mis grandes amigas Nati Barreto, Agus Berdini, Uara Carrillo y a Vale Ibañez Moro. A Ramiro Simonetti y a Matias Benavidez. A las Taloca por la aventura musical y a las Mwanamke-mbe, porque los fuegos eternos no se apagan. Al candombe y a la vida. A Let it Brew. A toda la banda que me bancó siempre.

A mi familia por su apoyo incondicional: mi mama Elba, mi papá Ubaldo, mi hermana Cecilia y mi tía Cristina. A mi abuela Nelly. A Chimi y a Volcán, mis compañeros de otra especie.

A CONICET por haberme otorgado una beca para la realización de este trabajo. A la Universidad Nacional de La Plata y a la Facultad de Ciencias Naturales y Museo por mi formación de grado y posgrado. Al Instituto de Limnología "Dr. Raúl A. Ringuelet" por el espacio físico y los recursos. A los organismos de financiamiento de Ciencia y Técnica estatales con cuyos fondos se realizó este trabajo de tesis.

A la Asamblea de Ciencia y Técnica de La Plata por la lucha y la organización. A ATE Conicet y JCP por defender nuestros derechos como trabajadorxs. A mis compañerxs de Ciencia en Movimiento por bancar todas las movidas. Al Estado Argentino por financiar el desarrollo de la Ciencia Pública y la Educación estatal, laica, diversa, libre y gratuita. Porque sin Ciencia Pública no hay Soberanía.

A todxs, muchas gracias. 


\section{ÍNDICE}

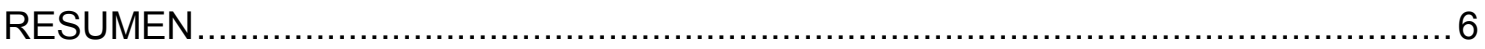

ABSTRACT (1)

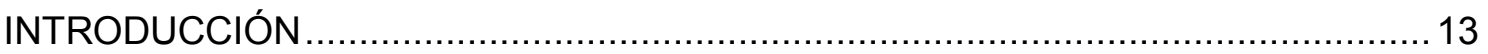

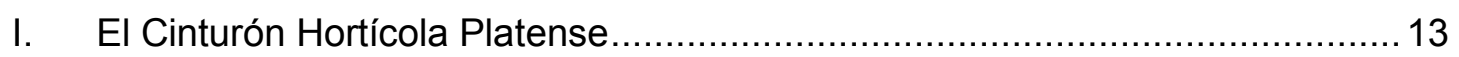

II. Uso intensivo de agroquímicos ............................................................... 16

III. Herramientas de diagnóstico y evaluación ................................................. 19

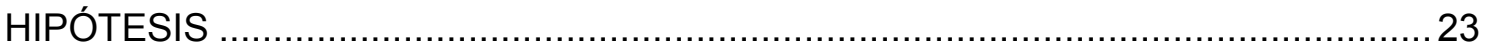

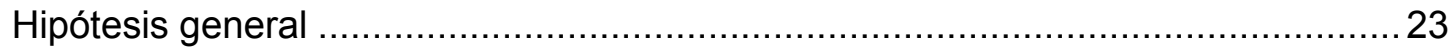

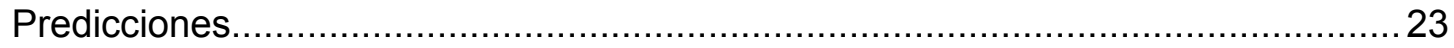

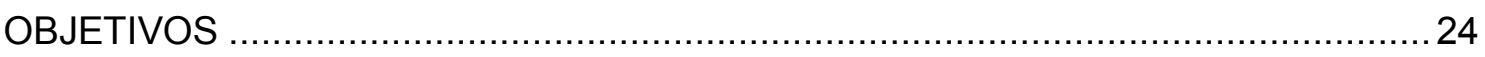

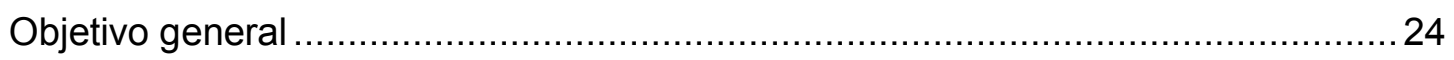

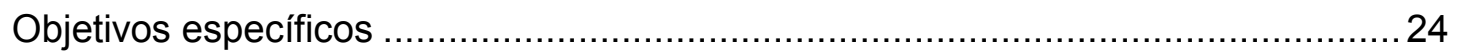

CAPÍTULO 1: Simocephalus vetulus como organismo modelo para ensayos de

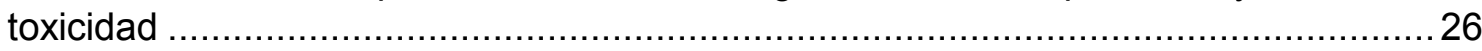

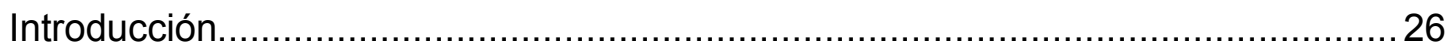

1.1. Ciclo de vida de Simocephalus vetulus....................................................... 30

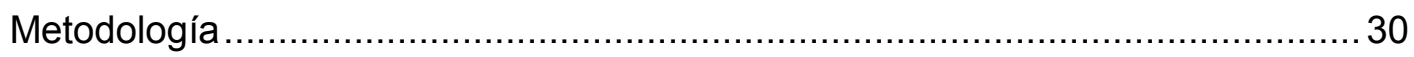

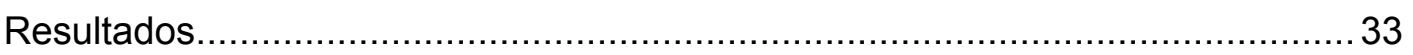

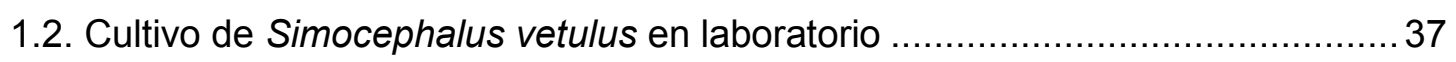

1.2.1. Metodología utilizada para el mantenimiento de Simocephalus vetulus .....38

1.2.2. Metodología utilizada para la separación de neonatos $<24 \mathrm{hs}$..................... 38

1.3. Ensayos de toxicidad y sensibilidad de Simocephalus vetulus ......................... 39

1.3.1. Determinación de CL50 de plaguicidas para Simocephalus vetulus........... 39

1.3.2. Sensibilidad de Simocephalus vetulus en relación a otros crustáceos .......42

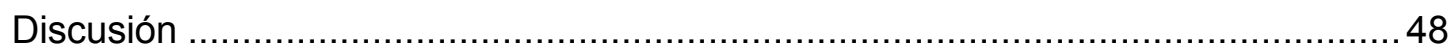

CAPÍTULO 2: Efectos sub-letales de la exposición a pulsos de toxicidad de plaguicidas

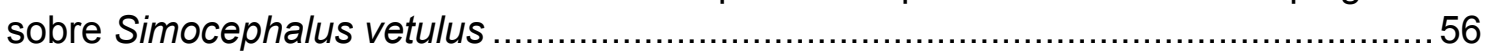

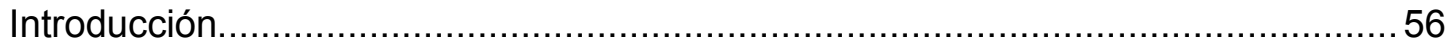

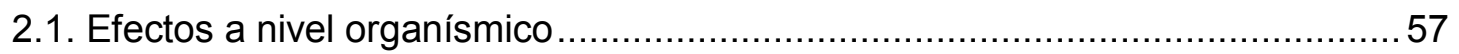

Diseño Experimental I: supervivencia, crecimiento y reproducción. ......................57

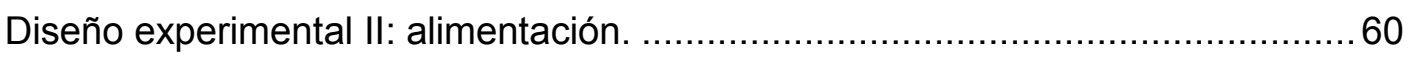

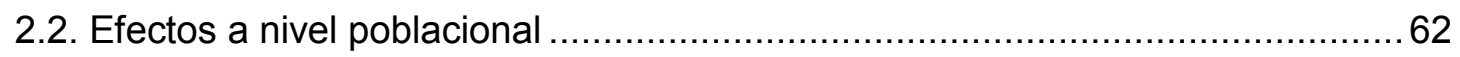

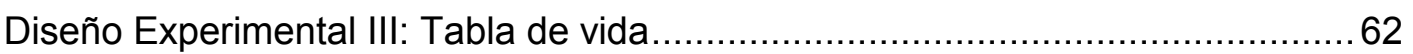

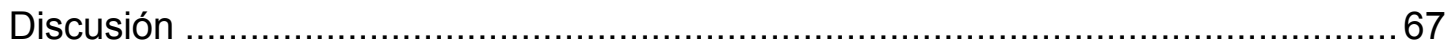

CAPÍTULO 3: Análisis del ensamble de invertebrados en cuencas con horticultura intensiva 


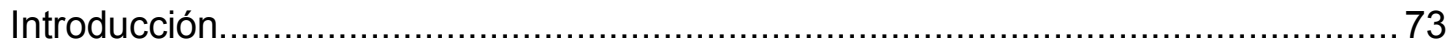

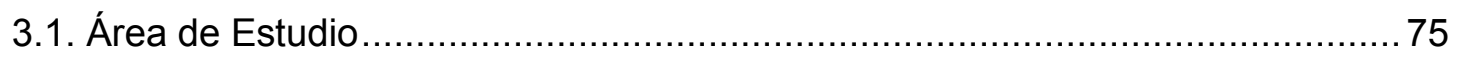

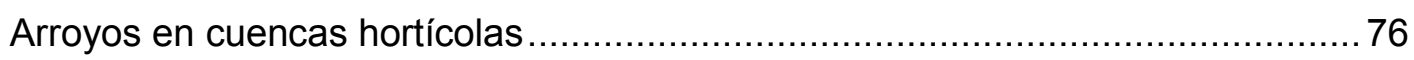

Arroyos de referencia: sin horticultura en sus cuencas ..................................... 79

3.2. Análisis del ensamble de invertebrados asociados a vegetación...................... 81

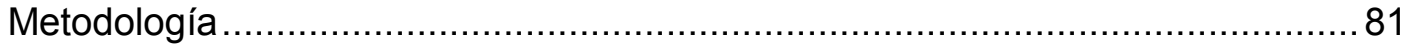

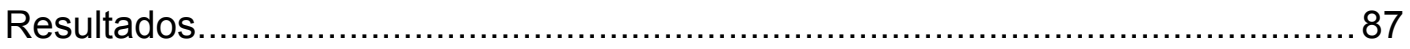

3.3. Comparación entre ambos periodos ..................................................... 107

3.4. Influencia de la vegetación y el uso del suelo sobre los ensambles................ 108

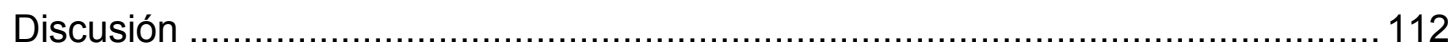

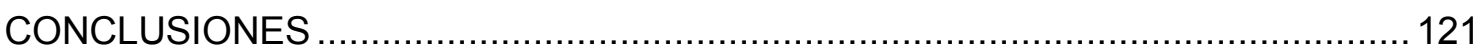

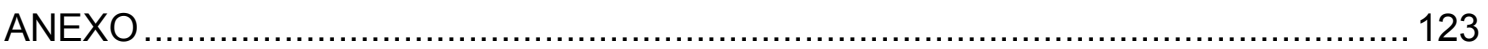

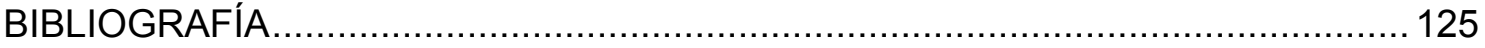




\section{RESUMEN}

El Cinturón Hortícola Platense (CHP) se ubica en el noreste de la Provincia de Buenos Aires, presenta una superficie de 3.709 hectáreas, una producción de 78.000 toneladas/año y abastece de verduras a 14 millones de personas; se considera una de las regiones productivas más importante del país. El modelo de producción hortícola responde a un modelo dependiente de insumos, basado en la implementación de invernaderos y uso intensivo de agroquímicos. El uso de plaguicidas en Argentina se incrementó de 39 millones de kilogramos en 1991 a 336 millones de kilogramos en el 2011, y el consumo de fertilizantes de 400 mil toneladas en los años 90' a más de 3 millones de toneladas en 2013. En el CHP se utilizan más de 160 productos diferentes, los insecticidas representan aproximadamente el $50 \%$ del total de los agroquímicos utilizados.

El objetivo del presente trabajo de tesis es estudiar el efecto del uso de agroquímicos sobre organismos acuáticos en arroyos de cuencas con actividad hortícola intensiva del Cinturón Hortícola Platense.

Se estudió el ciclo de vida de Simocephalus vetulus con el objetivo de establecer un cultivo semipermanente para esta especie y poder desarrollar experimentos de toxicidad en laboratorio. Se realizó un ensayo de crecimiento bajo dos temperaturas contrastantes, se determinaron los parámetros poblacionales y se establecieron metodologías de mantenimiento y separación de neonatos para los ensayos. Se determinó la concentración letal 50\% (CL50) de los plaguicidas más utilizados: cipermetrina, lambdacialotrina, clorpirifos, y glifosato (principios activos y formulados comerciales) en S. vetulus. Estos valores, en ocasiones menores a las concentraciones registradas en la bibliografía en arroyos pampeanos, sugirieren la ocurrencia de efectos agudos sobre la población residente de S. vetulus. Se comparó la sensibilidad de $S$. vetulus con otras especies de crustáceos para evaluar su utilidad como modelo para ensayos de toxicidad. La sensibilidad de $S$. vetulus fue mayor que organismos comúnmente utilizados como indicadores de contaminación en el hemisferio norte, como por ejemplo Daphnia magna, para todos los compuestos. En particular, S. vetulus mostró la menor CL50 para lamdacialotrina y glifosato de las especies de distribución local. Simocephalus vetulus resulta de gran utilidad en la realización de ensayos de toxicidad y como modelo para la evaluación de riesgo en los ambientes pampeanos. 
Se realizaron ensayos para evaluar los efectos de repetidos pulsos efímeros de toxicidad de lambdacialotrina y cipermetrina (formulados comerciales) sobre $S$. vetulus a nivel organísmico y poblacional. Se realizaron tres experimentos, los organismos fueron expuestos durante 60-90 minutos a diferentes concentraciones de lambdacialotrina o cipermetrina, fueron transferidos a agua limpia y se realizó un seguimiento diario. Estas exposiciones breves se repitieron cada 7 días a lo largo de los experimentos. Se determinó que $S$. vetulus sobrevive a pulsos efímeros de exposición a concentraciones hasta 5 veces mayores a los valores de CL50 de lambdacialotrina y cipermetrina, pero se registran efectos posteriores. Se determinó que organismos expuestos a repetidos pulsos efímeros de lambdacialotrina presentaron una disminución de la longevidad posterior a la exposición. Organismos expuestos a repetidos pulsos breves de cipermetrina presentaron menor tasa de alimentación, fecundidad acumulada, tasa de reproducción y tasa de incremento poblacional $(r)$ y, exceptuando este último parámetro, los efectos se observaron en organismos expuestos a concentraciones hasta 10 veces menores a los valores de CL50. Los resultados indican que una población residente, expuesta a pulsos efímeros de toxicidad disminuirá su densidad, y que distintas especies de invertebrados se verán afectadas de manera diferencial debido a su diferente sensibilidad, resultando en cambios en la estructura de la comunidad.

Se estudió el ensamble de invertebrados asociados a la vegetación, las concentraciones de nutrientes en agua y plaguicidas en sedimento y el porcentaje de horticultura en la cuenca de 4 arroyos ubicados en el Cinturón Hortícola Platense: San Juan, Martín, Gato y El Sauce, y se compararon con otros cuatro arroyos considerados como arroyos de referencia, (sin actividad hortícola en sus cuencas): Sin Nombre, Buñirigo, Morales y Destino, los dos primeros se localizan en cuencas con uso del suelo ganadero sobre pasturas naturales y los 2 últimos ubicados en la reserva de la biosfera Parque Costero del Sur. Se realizaron en total 11 muestreos en la época de mayor aplicación de agroquímicos de Octubre de 2014 a Marzo de 2017.

Las concentraciones de nutrientes en los arroyos hortícolas fueron significativamente mayores que en los arroyos de referencia, y estuvieron positivamente correlacionadas con el porcentaje de horticultura en la cuenca. Las concentraciones y la frecuencia de detección de plaguicidas fue mayor en arroyos hortícolas, y las concentraciones de plaguicidas se correlacionaron con las concentraciones de nutrientes. Estos resultados sugieren un origen común: las aplicaciones en los cultivos adyacentes, que son incorporadas a los arroyos en coincidencia con los eventos de escorrentía superficial posteriores. 
Los ensambles de invertebrados asociados a la vegetación en arroyos hortícolas fueron significativamente diferentes a los ensambles de los arroyos de referencia. Los sitios hortícolas presentaron menor riqueza y densidad de invertebrados y ensambles caracterizados por colémbolos (principalmente Entomobryoidea), Dugessidae e Hirudinea, mientras que los arroyos de referencia se caracterizaron por su mayor riqueza y densidad y mayor abundancia de $S$. vetulus, Hyalella y Baetidae. Trichoptera, Decapoda y Caenis, considerados sensibles a los plaguicidas, aparecieron sólo en sitios de referencia. La riqueza y la diversidad se correlacionaron negativamente con las concentraciones de nutrientes y el porcentaje de horticultura en la cuenca. Del mismo modo, la riqueza se correlacionó negativamente con las concentraciones de plaguicidas. La abundancia de S. vetulus, Hyalella y Baetidae, además, se correlacionaron negativamente con el porcentaje de horticultura en la cuenca, mientras que la de Entomobryoidea y Dugessidae se correlacionaron positivamente. Se concluye que el uso de agroquímicos en la horticultura modifica la composición y la estructura del ensamble de invertebrados por pérdida de organismos sensibles y aumento de la densidad de grupos taxonómicos tolerantes. Las concentraciones de nutrientes en los arroyos, la menor riqueza de taxones y la abundancia de $S$. vetulus, Hyalella y Baetidae son buenos indicadores de impacto por agroquímicos. 


\section{ABSTRACT}

The "Cinturón Hortícola Platense" (CHP) is located in the northeast of Buenos Aires province, the area measures 3,746 hectares, produces 78,360 tons per year and supplies vegetables for 14 million people; it is considered one of the most important productive region in the country. The horticulture production system response to an input-dependent cropping system based on greenhouse technology and agrochemicals intensive use. Pesticide use has increased from 39 million kilograms in 1991 to 336 million in 2011, and fertilizer consumption from 400,000 to above 3 million tons in 2013. One hundred and fifty different products are being used; insecticides represent roughly $50 \%$ of the total consumption.

The aim of the current study is to assess the effect of agrochemical use on aquatic organisms in streams with horticultural basins from the Cinturón Hortícola Platense.

The life cycle of Simocephalus vetulus was studied in order to establish a semipermanent culture for this specie and to develop laboratory toxicity essays. A growth essay was performed under two different temperatures, population parameters were determined and maintenance and neonates handling procedures for toxicity tests were stablished. The lethal concentration $50 \%$ (LC50) of the most commonly used pesticides: cypermethrin, lambdacyhalothrin, chlorpyrifos and glyphosate (active ingredients and formulations) for $S$. vetulus were determined. The LC50 were occasionally lower than the reported concentrations in the literature for pampean streams, suggesting the occurrence of acute effects over the resident population of $S$. vetulus. The sensitivity of $S$. vetulus was compared with the sensitivity of other crustacean species in order to evaluate its suitability as model organism for toxicity testing. The sensitivity of $S$. vetulus was higher than that for other commonly used species, such us Daphnia magna, for all compounds. In particular, S. vetulus showed the lowest LC50 value for lambdacyhalothrin and glyphosate of the local species. Simocephalus vetulus is suitable for toxicity testing and as model species for risk assessment in pampean streams.

Bioessays were performed in order to assess the effects of repeated ephemeral toxicity pulses of lambdacyhalothrin and cypermethrin (formulations) on $S$. vetulus at individual and population levels. Three experiments were conducted; organisms were exposed 60-90 minutes to different concentrations of lambdacyhalothrin or 
cypermethrin, transferred to clean water and counted daily. These short expositions were repeated every 7 days along the experiment. Simocephalus vetulus survived to pulse exposures at concentrations up to five times the lambdacyhalothrin or cypermethrin LC50 values, but showing later effects. Organisms exposed to repeatedephemeral toxicity pulses of lambda-cyhalothrin showed decreased longevity following expositions. Organisms exposed to repeated ephemeral toxicity pulses of cypermethrin showed lower feeding rate, cumulative fecundity, reproduction rate and population increase rate ( $r$ ), and except for the latter, these effects were registered in organisms exposed to concentrations up to 10 times lower than the LC50 values. Present results indicate that a resident population exposed to ephemeral toxicity pulses would decrease its density, and different species would be affected differently because of its different sensitivity, resulting in changes on community structure.

The assemblage of macrophyte-associated invertebrates, nutrient concentrations in water, pesticide concentrations in sediments and horticulture land use proportion in the basin were studied in four streams located in the CHP: San Juan, Martín, Gato and Sauce; and were compared with another four considered as reference streams (without horticulture land use in their basins): Sin Nombre, Buñirigo, Morales and Destino, the first two located in extensive livestock basins and the other two located in a UNESCO Biosphere Reserve "Parque Costero del Sur". A total of 11 samplings were performed in the main agrochemicals application period, from October 2014 to March 2017.

Nutrient concentrations in horticultural streams were significantly higher than in the reference streams, and were positive correlated to the horticulture percentage in the basin. Pesticide detection frequency and concentrations were higher in horticultural streams, and pesticides concentrations were correlated to nutrient concentrations. These results suggest a common source: adjacent crop applications entering into streams in coincidence with surface runoff events.

Invertebrate assemblages in horticultural streams were significantly different to assemblages of reference streams. Horticultural sites showed lower richness and density, and assemblages characterized by Collembola (mainly Entomobryoidea), Dugessidae and Hirudinea, while reference sites showed higher richness and density, and higher abundances of S. vetulus, Hyalella and Baetidae. Trichoptera, Decapoda and Caenis, considered sensitive to pesticides, appeared only in reference streams. Richness and diversity were negatively related to nutrient concentrations and horticulture percentage in the basin. In the same way, richness was negatively related to pesticide concentrations. Also, S. vetulus, Hyalella y Baetidae were negatively related to horticulture percentage in the basin, while Entomobryoidea and Dugessidae 
were positively related. It is concluded that agrochemical use in horticulture changes the invertebrate assemblage structure and composition because of the loss of sensitive species groups and the increase of tolerant taxones. Nutrient concentrations in streams, lower taxa richness and abundances of $S$. vetulus, Hyalella and Baetidae are suitable indicators of agrochemical impact in pampean streams. 
INTRODUCCIÓN 


\section{INTRODUCCIÓN}

\section{El Cinturón Hortícola Platense}

El periurbano es el área de transición entre la zona urbana y la zona rural, es un territorio productivo, residencial y de servicios que se sitúa alrededor de las ciudades; dentro del periurbano se emplazan los denominados cinturones verdes, donde se desarrolla la horticultura (Barsky 2010). En Argentina se estima una producción hortícola anual de entre 8 y 10 millones de toneladas en una superficie aproximada de 500.000 hectáreas, representando el $1,5 \%$ de la superficie productiva del país (Fernández Lozano 2012). El principal destino de la producción hortícola es el mercado interno, abasteciendo de verduras y hortalizas frescas a la población durante todo el año.

El Cinturón Hortícola Platense (CHP) se ubica en el partido de La Plata dentro de la región bonaerense, pertenece al Cinturón Verde o Área Hortícola Bonaerense, y linda con las regiones productivas de Florencio Varela y Berazategui. Las principales hortalizas cultivadas en el CHP son (por orden de cantidad de producción): lechuga, tomate, alcaucil, acelga, espinaca, choclo, pimiento, brócoli y repollo, entre otras (Sarandón et al. 2015).

El Cinturón Hortícola Platense comenzó a desarrollarse en el año 1882 junto con la fundación de la ciudad de La Plata, donde se delimitaron el casco urbano y la zona de quintas y chacras en los alrededores de la ciudad. Entre fines del siglo XIX y principios del siglo XX llegaron inmigrantes europeos, entre ellos, españoles, portugueses e italianos, que constituyeron las poblaciones núcleo del cinturón. El crecimiento del CHP estuvo directamente relacionado con el aumento demográfico que tuvo lugar en las ciudades desde la década del '30 al '60 debido a políticas favorables de empleo en las mismas, y a la reducción de la demanda de mano de obra en el campo, entre otras (García 2010). Las poblaciones urbanas en ascenso generaron cada vez más demanda de alimentos frescos para el consumo cotidiano (Villulla 2006). En los '70 se produce una expansión generalizada de la actividad por profundas transformaciones tecnológicas (mecanización, agroquímicos, híbridos, riego localizado) (García 2010) y por una gran inmigración de horticultores bolivianos (Barsky y Vio 2007). A partir de la década del '90 se incorporó la técnica del invernáculo, permitiendo un mayor rendimiento, calidad, seguridad de la cosecha y 
competencia económica, lo que le permitió al CHP diferenciarse ante un mercado sobreofertado (García 2010).

En la actualidad, los datos oficiales disponibles sobre la superficie y producción del Cinturón Hortícola Platense corresponden al Censo Hortiflorícola Bonaerense del año 2005 (CHFB 2005), donde se determinó una superficie de 3709 ha y una producción de 78360 toneladas por año; la superficie bajo cubierta corresponde al $80 \%$ del total de la superficie del Cinturón Verde Bonaerense, del cual forma parte. En los últimos años se observa un incremento de la superficie cultivada y del número de invernaderos construidos pero no se cuenta con información actualizada. Según estimaciones, representa el $60-70 \%$ de la producción de la región bonaerense, fundamentalmente la producción bajo cubierta (Argerich y Troilo 2010, García 2011). El Cinturón Hortícola Platense produce entre el $60 \%$ y $90 \%$ de la verdura fresca que consume la Ciudad Autónoma de Buenos Aires y alrededores (Benencia 2002), estimado en 14 millones de personas; esto hace que sea considerado como la región de producción más importante del país (García 2012) junto con el de General Pueyrredón (Sarandón et al. 2015).

Como contraparte, el Cinturón Hortícola Platense, como muchas regiones frutihortícolas del país, está inmerso en una profunda problemática social y económica. La producción hortícola se realiza principalmente por agricultores familiares, que comprenden entre 5.000 y 9.000 familias (Defensoría del Pueblo 2018) y la mayoría reside en la zona de producción (según el CHFB, el $85 \%$ de los productores viven y trabajan en el establecimiento).

Una de las principales problemáticas es la falta de acceso a la tierra. El último censo indicó que más del $50 \%$ de los establecimientos hortícolas de La Plata son arrendados (CHFB 2005). El crecimiento de la población y la constante expansión del área urbana producen un desplazamiento del área agrícola hacia las periferias y se produce una revalorización de la tierra. Barsky (2005) denomina a la zona periurbana como "cinturones de especulación inmobiliaria", ya que su valor está sujeto al mercado inmobiliario y produce que, por ejemplo, el monto del arrendamiento en La Plata sea mayor al del resto de la región hortícola bonaerense (García 2014). Este es uno de los principales aspectos que condicionan las posibilidades de mejoramiento de las condiciones habitacionales, la planificación de los cultivos y la transición hacia un modelo de producción más sustentable.

Otra problemática es la condición de vida y trabajo de los agricultores familiares (García y Lemmi 2011). Según García (2015) el éxito del modelo productivo del Cinturón Hortícola se debe no sólo a la incorporación de la técnica de invernáculo, sino 
que se sustenta en la total precarización de sus trabajadores: trabajos no formales con salarios muy bajos y con nulas condiciones de infraestructura (servicios de luz, agua, pavimentación, recolección de residuos). Sumado a esto, las viviendas son precarias, no hay acceso a servicio de agua potable o cloacas (Moreyra et al. 2012), y además, los trabajadores y sus familias están en contacto directo con los agroquímicos que son utilizados en los cultivos, lo que incrementa el riesgo para su salud.

A lo anterior, se suma la problemática de la comercialización, y particularmente el problema del transporte de la producción. El circuito comercial comprende varios actores: los productores, los intermediarios, el mercado concentrador, el comercio minorista y los consumidores (Caracciolo 2016). La mayoría de productores no tienen los medios para el transporte de la cosecha hacia los mercados, por lo que dependen de la venta "a culata de camión", vendiendo la producción a intermediarios quienes la venden al mercado concentrador y son quienes establecen el precio (Defensoría del Pueblo 2018); esta modalidad representa el 64\% de la comercialización (Duré 2014) y conlleva al incremento en el precio de los productos y menores ganancias para los productores.

En los últimos años se establecieron algunas políticas públicas para el desarrollo de la agricultura familiar y para promover mejores condiciones de trabajo y de vida. Por ejemplo, en el año 2009 se implementó el Monotributo Social Agropecuario: las familias productoras podían registrarse como trabajadores formales pudiendo acceder a una obra social y aportes jubilatorios, cuyo costo era absorbido por Desarrollo Social y por el Ministerio de Agricultura. En el año 2014 se creó la Secretaría de Agricultura Familiar para promover políticas para esta actividad (capacitaciones, desarrollo rural, promoción de economías regionales) (Boletín Oficial 2014), y en el año 2015 se promulgó la Ley $N^{\circ} 27.118$ de Reparación Histórica de la Agricultura Familiar (Infoleg 2015) donde se declara de interés público la agricultura familiar, campesina e indígena.

Pese a los avances mencionados anteriormente, en la actualidad la problemática continúa vigente. En el año 2018 se reduce el aporte estatal del Monotributo Social Agropecuario y se disuelve la Secretaría de Agricultura Familiar mediante el decreto 302/17 (Boletín Oficial 2018). Las organizaciones de productores plantean que la falta de acceso a la tierra es un impedimento para la implementación de una producción sustentable ya que los contratos de alquiler son de pocos años, impidiendo la planificación a largo plazo de los cultivos. Además, el costo elevado de la tierra promueve la intensificación del uso del suelo y una mayor demanda de insumos, para asegurar una cosecha que sustente el alquiler y los costos de producción (MTE Rural 
2017, UTT 2018). La Defensoría del Pueblo de la Provincia de Buenos Aires en su informe reciente (2018) reafirma que la dificultad del acceso a la tierra promueve la especulación inmobiliaria perjudicando a los productores, a los consumidores y ciudadanos. Esto se vincula con la calidad de vida de las familias agricultoras, el modo intensivo de producción y el deterioro ambiental.

\section{Uso intensivo de agroquímicos}

Se denomina agroquímico a cualquier compuesto o sustancia o mezcla de sustancias naturales o sintéticas utilizadas para controlar o evitar la acción de plagas o enfermedades agrícolas, incrementar el crecimiento de las plantas y proteger el producto cosechado, que permiten aumentar el rendimiento de la cosecha y la oferta de alimentos (García y Lazovski 2011). En este trabajo de tesis se considerarán agroquímicos tanto los plaguicidas (insecticidas, herbicidas, fungicidas) como los fertilizantes.

El uso de agroquímicos en Argentina se incrementó durante los últimos años. EI uso de plaguicidas aumentó de 39 millones de kilogramos en 1991 (Moltoni, 2012) a 336 millones de kilogramos en el 2011 (CASAFE 2013). El consumo de fertilizantes siguió la misma tendencia, de 400.000 toneladas en los años 90' a más de 3 millones de toneladas en 2013 (CIAFA 2017). Si bien estos datos corresponden en mayor medida a la agricultura extensiva (soja, trigo, maíz), el modelo de producción hortícola responde a un modelo generalizado de producción con un manejo altamente dependiente de insumos.

La implementación del invernadero en la horticultura permitió ampliar la oferta de productos y el número de ciclos por año (hasta 6 en el caso de las hortalizas de hoja), lo que implicó un aumento generalizado en la frecuencia de aplicación tanto de fertilizantes como de pesticidas. A diferencia de la agricultura extensiva, donde el $87 \%$ de los agroquímicos más utilizados son herbicidas (el 65\% corresponde al glifosato), en la horticultura se utiliza una mayor proporción de insecticidas, cercano al $52 \%$ (Pacheco y Barbona 2017).

En el estudio realizado por la Defensoría de Pueblo de la Provincia de Buenos Aires (Sarandón et al. 2015) se determinó que en el Cinturón Hortícola Platense se aplican como fertilizantes urea, fosfato di o monoamónico, sulfato de calcio y diferentes mezclas. En cuando a plaguicidas se utilizan más de 160 productos, considerando herbicidas, insecticidas y fungicidas. Por ejemplo, en el cultivo de 
tomate, se utilizan más de 60 principios activos diferentes como carbofuran, clorpirifos, cipermetrina, diazinon, tiametoxam, spinosad, entre otros; y junto al morrón son los cultivos de la zona que más productos utilizan. Dicho informe expone que los cultivos bajo cubierta presentan mayor índice de peligrosidad que los cultivos al aire libre, basándose en la cantidad, frecuencia y categoría toxicológica del principio activo utilizado. Además, señala que este tipo de producción intensiva genera un impacto más negativo para el hábitat y la vida silvestre en comparación con otras actividades productivas (ej. ganadería extensiva).

En Argentina la elaboración, comercialización, distribución, almacenamiento y aplicación de agroquímicos se rigen por diferentes leyes, decretos y resoluciones de organismos estatales como el ex Ministerio de Agricultura, Ganadería y Pesca (actual Ministerio de Agroindustria), el ex Ministerio de Salud (actualmente Secretaría dependiente del Ministerio de Desarrollo Social), la Secretaría de Ambiente y Desarrollo Sustentable y el Servicio Nacional de Sanidad y Calidad Agroalimentaria (SENASA). Estos organismos también son responsables de las evaluaciones y registro de los distintos principios activos, de la autorización y control, y de las prohibiciones o restricciones de uso que correspondieran (Pórfido 2014). Particularmente en la provincia de Buenos Aires rigen la Ley Provincial Nº 10699 (1998) y el Decreto 499/91 (1991) (Manual Fitosanitario 2018), donde se indica que los productos fitosanitarios (es decir, agroquímicos) deben presentar una hoja de seguridad que indique la forma de uso, precauciones y peligrosidad y debe estar registrado y regulado por SENASA. Además, está prohibida la utilización y aplicación de productos sin una receta agronómica, la cual debe indicar tipo de producto, dosis, aplicación, condiciones del cultivo y condiciones climáticas. Esta legislación carece de control y resulta inaccesible a productores de bajos recursos, por lo que el manejo se realiza de manera convencional, con aplicaciones por calendario o por asesoramiento de los comerciantes y/o empresas de agroquímicos (Sarandón et al. 2015).

Los plaguicidas más utilizados corresponden a los insecticidas cipermetrina (piretroide) y clorpirifos (organofosforado), y al herbicida glifosato. Los piretroides son sustancias sintéticas similares a las piretrinas, insecticidas naturales presentes en los crisantemos (Chrysantemum sp.), actúan como insecticidas de contacto que, una vez aplicados, no presentan movilidad dentro de la planta (Kahl 2013). Actúan sobre el sistema nervioso central, inhiben el cierre de los canales de sodio que generan el gradiente de carga del axón, impiden que la membrana se polarice y que vuelva al estado basal. Esto produce una actividad repetitiva en órganos sensoriales y según el tipo de piretroide, también en terminales nerviosas motoras y fibras musculares 
esqueléticas, produciendo finalmente una parálisis en el organismo y posteriormente la muerte (Vijverberg y van den Bercken 1990).

Los organofosforados son insecticidas de ingestión e inhalación, son absorbidos por la planta (cultivo) y actúa en los insectos plaga cuando los ingieren. Actúan sobre el sistema nervioso central, inhiben la enzima acetilcolinesterasa, encargada de degradar la acetilcolina, neurotransmisor responsable de propagar el impulso nervioso. Al inhibir la enzima de degradación, la acetilcolina se acumula y genera distintos efectos, como contracción muscular, depresión de la función motora y respiratoria (Reigart y Roberts 1999). Se produce una falla respiratoria y cardíaca, y posteriormente la muerte. Tienen una baja persistencia en el medioambiente pero son altamente tóxicos para mamíferos, aves y peces, entre otros (Kahl 2013).

El glifosato es un fosfonato utilizado como herbicida no selectivo, se utiliza generalmente en forma de la sal $\mathrm{N}$-fosfometilglicina. Es el producto activo del formulado Round Up® de Monsanto (2018) que se utiliza desde el año 1970 en todo el mundo. Su modo de acción es inhibir la enzima enolpiruvilsiquimato-3-fosfatosintasa (EPSPS), que se encuentra dentro de los cloroplastos y está implicada en la formación del ESP (precursor de aminoácidos aromáticos), por lo que su inhibición afecta la producción de proteínas, hormonas, vitaminas y otros metabolitos, provocando la muerte de la planta por falta de nutrientes. El sitio activo de la enzima EPSPS es muy constante en plantas superiores y está ausente en animales, lo cual convierte al glifosato en un herbicida de amplio espectro. El glifosato forma complejos estables con los cationes en solución acuosa y es inmovilizado por adsorción en el suelo, por lo que el tiempo de residencia depende de la composición del suelo (Camino y Aparicio 2010). Se ha demostrado que su producto de degradación, el ácido aminometilfosfónico (AMPA), presenta una alta toxicidad para invertebrados (Giesy et al. 2000) y efectos clastogénicos en linfocitos humanos (Mañas et al. 2009).

Los agroquímicos utilizados en los cultivos pueden llegar a los arroyos por diferentes vías: accidental; por aplicación directa, cuando se utilizan herbicidas para eliminar "malezas" de los canales de drenaje o de los bordes de los arroyos; por deriva por el viento, ya que pueden haber pulverizaciones cercanas a los cuerpos de agua o aplicaciones aéreas; por escorrentía superficial, provocada por lluvias luego de las aplicaciones en los cultivos. Esta última es considerada una de las principales fuentes de contaminación por plaguicidas en cuerpos de agua superficiales, produciendo pulsos de toxicidad para la fauna residente (Schultz 2001, Jergentz et al. 2005, Mugni et al. 2011). El ingreso de los fertilizantes aumenta las concentraciones de nitrógeno y fósforo del agua de arroyos cercanos a cultivos y promueve el proceso de 
eutrofización (Wetzel 1981), el incremento de la biomasa, cambios en la composición de especies de algas, fluctuaciones de $\mathrm{pH}$ y oxígeno disuelto, afectando la calidad del agua (Smith et al. 1999).

Al ingresar a los cuerpos de agua los plaguicidas afectan a los sistemas en diferentes niveles de organización (Schäfer et al. 2011). Actúan sobre los organismos a nivel fisiológico (ej., cambios en la actividad enzimática o tasa metabólica), pudiendo derivar en un efecto individual (alteraciones en el crecimiento o en la reproducción). Si el efecto es importante y afecta a varios individuos, puede propagarse a nivel poblacional (cambio en la estructura de edades o en la tasa de crecimiento poblacional), incluso causando la desaparición o reemplazo de alguna especie (Egler et al. 2012, Liess et al. 2008, Schäfer et al. 2012); es así que pueden observarse cambios a nivel de la estructura de la comunidad y en los procesos ecosistémicos (Schäfer et al. 2012, Peters et al. 2013).

Los ambientes acuáticos brindan numerosos servicios para la población: provisión de agua, energía, alimentos y recursos genéticos, purificación de agua, regulación del flujo, y también servicios culturales como educación, recreación, valor estético y sentido de pertenencia (Harrison et al. 2010). La modificación en las funciones de los ecosistemas puede afectar finalmente a los servicios ecosistémicos. Por ejemplo, el uso del suelo en las zonas altas puede afectar a las zonas bajas, reduciendo la disponibilidad de agua potable en centros urbanos (Jobággy 2011) y se puede alterar el régimen hidrológico causando la pérdida de naturalidad y conectividad

y limitando la regulación de las inundaciones (Gómez et al. 2016). En particular, el uso de agroquímicos puede alterar la calidad del agua, tanto superficial como subterránea (Carpenter et al. 1997, Harrison et al. 2010), afectar la dinámica de descomposición de la materia orgánica modificando los niveles energéticos de los cuerpos de agua (Schäfer et al. 2012) y causar un riesgo potencial para la salud humana y ambiental (Sarandón et al. 2015).

\section{Herramientas de diagnóstico y evaluación}

Los estudios de riesgo ambiental se basan en general en datos provenientes de la determinación de la concentración letal $50 \%$ (CL50) de cierto compuesto para diferentes especies, llevada a cabo en condiciones definidas. La CL50 indica la concentración de un compuesto a la cual muere el $50 \%$ de los individuos expuestos luego de un cierto tiempo de exposición (Trautmann et al. 2001). Si bien estos estudios 
resultan alejados de un escenario ambientalmente realista, son utilizados como información de base para desarrollar trabajos posteriores y como una primera aproximación de los efectos de los compuestos sobre los organismos.

Existen protocolos estandarizados para llevar a cabo estas determinaciones (USEPA 1996, OECD 1998, OECD 2000, USEPA 2000, USEPA 2002) elaborados por organismos gubernamentales de diferentes países. Muchas de las especies utilizadas en ellos no están presentes en nuestro medio: cladóceros de las especies Daphnia magna Straus 1820 y D. pulex Leydig 1860, y anfípodos como Gammarus pulex (L. 1758) y Hyalella azteca (Saussure 1858). La toxicidad de un compuesto para esos organismos resulta de utilidad relativa en estudios de impacto ambiental para la fauna local, por lo que es importante evaluar el impacto de los agroquímicos con especies que sean sensibles y representativas de la fauna característica de los ambientes regionales.

Para comprender el impacto de los agroquímicos en los ambientes naturales es necesario realizar estudios en condiciones ambientales complejas y realistas, donde las poblaciones interactúen entre sí, estén sometidas a otros factores que actúen simultáneamente y varíen en el tiempo y el espacio (Schulz 2004). En ese sentido, el estudio a campo de las comunidades acuáticas es importante y muy utilizado para evaluar el estado de los cuerpos de agua.

Asimismo, es improbable abarcar una comunidad en su totalidad por lo que en estudios ecológicos se restringe el estudio a un grupo en particular (Begon et al. 2006), definido a nivel taxonómico (ej. peces) o por su hábitat (ej. zooplancton). Los ensambles se definen como un conjunto de individuos de distintas especies sometidos a un esfuerzo de muestreo en un punto o área determinados (Magurran y McGill 2011). Un ensamble cuya composición refleja las condiciones ambientales puede actuar como bioindicador del estado del ecosistema (Gerhardt 2002). En particular, los ensambles de invertebrados han sido reconocidos como uno de los más adecuados para evaluar el estado de los cuerpos de agua (Resh et al. 1995, Barbosa et al. 2001, Miserendino et al. 2011, Schäfer et al. 2011). La presencia/ausencia y la densidad de ciertos organismos pueden relacionarse con diferentes parámetros que caractericen la calidad del agua y así generar bioindicadores para evaluar el estado de los ambientes acuáticos (Barbour 1999, Miserendino y Pizzolón 1999, Rodrigues-Capítulo et. al, 2001, Liess y Van der Ohe 2005). Estudios en la región comprueban que los ensambles de invertebrados resultaron buenos indicadores del uso del suelo agrícola (Jergentz et al. 2004, Solis et al. 2016, Solis et al. 2017). 
La utilización de nuevos productos y la incorporación de nuevas tecnologías hacen que sea de suma importancia el estudio del impacto del uso de los mismos para adoptar normas de manejo adecuadas y establecer un modelo productivo con el menor riesgo ambiental posible. Relyea y Hoverman (2006) plantean que si se conocen los efectos letales y subletales se puede predecir cómo los plaguicidas cambian los atributos y densidades de las especies, y en consecuencia, cómo la comunidad y las funciones del ecosistema pueden verse modificadas. Moss (2008) señala que es importante estudiar de manera holística el impacto de las prácticas agrícolas ya que alteran a los ecosistemas en su conjunto. Es por eso que es importante realizar estudios en condiciones ambientalmente relevantes.

La propuesta del presente trabajo de tesis consiste en realizar estudios complementarios a escala de laboratorio y campo, combinando el estudio de los efectos de plaguicidas a nivel de organismos y poblaciones con el de ensambles de comunidades de invertebrados acuáticos asociados a vegetación en arroyos de la zona hortícola, lo que permitirá generar información acerca del efecto del uso de agroquímicos en los organismos residentes de dichos ambientes. 
HIPÓTESIS Y OBJETIVOS 


\section{HIPÓTESIS}

\section{Hipótesis general}

La utilización de agroquímicos en los cultivos del Cinturón Hortícola Platense afecta la calidad del agua y la estructura de los ensambles de invertebrados de los arroyos adyacentes.

\section{Predicciones}

1. La utilización de fertilizantes en cuencas donde su suelo se emplea con fines hortícolas intensivo contribuye al incremento de las concentraciones de nutrientes (nitrógeno y fósforo) y clorofila en las aguas superficiales.

2. Los arroyos de cuencas con cultivos hortícolas presentan concentraciones de plaguicidas en rangos que producen efectos letales y sub-letales para los invertebrados acuáticos.

3. Los pulsos de toxicidad de diferentes plaguicidas afectan la supervivencia, el crecimiento, y la reproducción de los organismos expuestos.

4. La resultante final de la aplicación de agroquímicos sobre una comunidad compleja, formada por organismos de muy diferente sensibilidad, es un cambio en la composición y en la estructura del ensamble por pérdida de organismos sensibles y aumento de la densidad de grupos taxonómicos tolerantes. 


\section{OBJETIVOS}

\section{Objetivo general}

Estudiar el efecto del uso de fertilizantes y plaguicidas sobre fauna de invertebrados en arroyos de cuencas con actividad hortícola intensiva del Cinturón Hortícola Platense.

\section{Objetivos específicos}

-Estudiar el ciclo de vida de Simocephalus vetulus O.F. Müller y desarrollar una metodología para su utilización en ensayos de toxicidad.

-Determinar la concentración letal (CL50) de los plaguicidas más utilizados en nuestro medio: clorpirifos, cipermetrina, lambdacialotrina y glifosato para el cladócero S. vetulus.

-Comparar las CL50 determinadas para S. vetulus con las de otras especies y con las concentraciones determinadas en los arroyos.

-Analizar los efectos de pulsos de toxicidad de los plaguicidas mencionados sobre parámetros del ciclo de vida y poblacionales de $S$. vetulus.

-Determinar las concentraciones de nutrientes y plaguicidas en arroyos de cuencas con cultivos hortícolas intensivos y de arroyos de cuencas no asociadas a prácticas hortícolas considerados de referencia.

-Caracterizar la estructura del ensamble de invertebrados acuáticos asociados a la vegetación en arroyos hortícolas y compararla con la de arroyos de referencia. 


\section{CAPÍTULO 1.}

Simocephalus vetulus como

organismo modelo para ensayos de toxicidad 


\section{CAPÍTULO 1: Simocephalus vetulus como organismo modelo para ensayos de toxicidad}

\section{Introducción}

Los cladóceros, comúnmente denominados "pulgas de agua", son crustáceos branquiópodos que habitan en los ambientes acuáticos. Son en general de tamaño pequeño, entre 0,2 y $3 \mathrm{~mm}$ (sólo Leptodora llega a $18 \mathrm{~mm}$ ) y es característico un caparazón univalvo comprimido lateralmente que encierra al cuerpo, pueden presentar una espina terminal o estar reducido. El cuerpo se divide en cabeza, que lleva un rostro y dos pares de antenas, y un tronco que presenta una segmentación reducida con el tórax y abdomen fusionados. Éste lleva de 4 a 6 pares de apéndices de tipo filopodiales, la parte posterior está recurvada y lleva un par de ganchos (fig. 1.1). La parte dorsal e interna del caparazón funciona como cámara de incubación. Las anténulas (primer par de antenas) son unirramosas con función sensorial y las antenas (segundo par) son birramosas generalmente con función natatoria. Es característico un único ojo compuesto central y un ocelo (Mc Laughlin 1980).

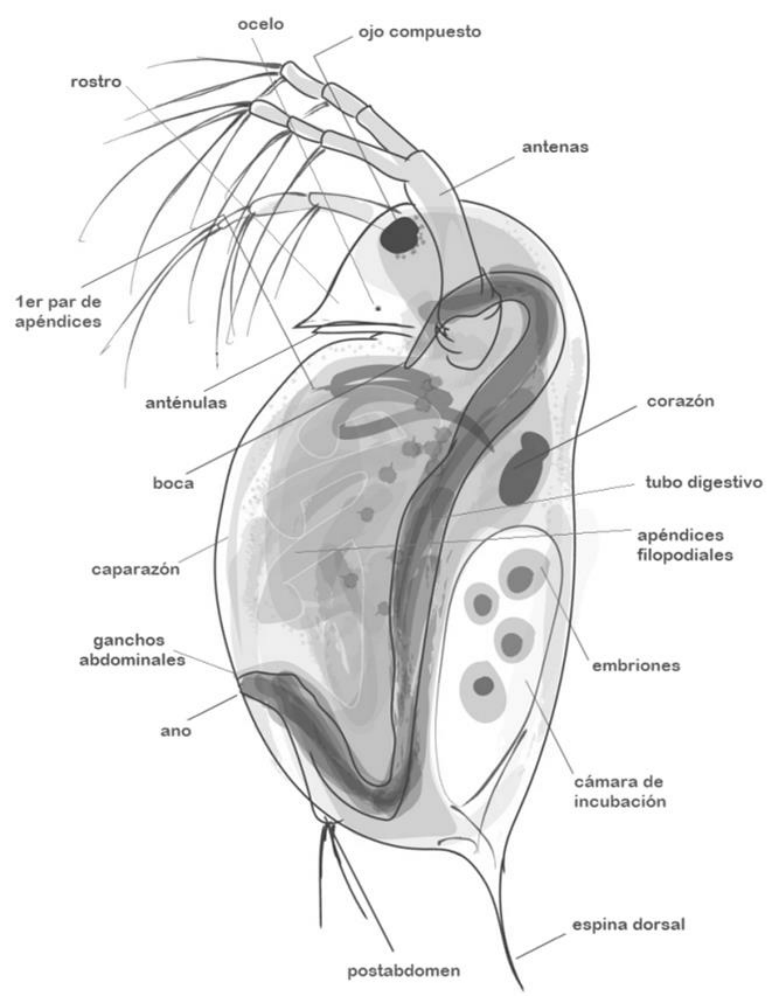

Figura 1.1. Esquema de un cladócero. Modificado de Mc Mahon (2016). 
Son organismos dioicos, la partenogénesis es la forma más común de reproducción y generalmente las poblaciones están dominadas por hembras. La producción de machos se da, en general, en condiciones desfavorables: aumento de la densidad poblacional, reducción del fotoperiodo, sequía, aumento ó disminución extrema de temperatura, reducción de la disponibilidad de alimento. En la reproducción sexual se producen huevos de resistencia denominados efipios que son capaces de resistir condiciones adversas (ej: bajas temperaturas, sequía), y dan lugar a una nueva población. Muchas especies presentan también cambios morfológicos estacionales, denominados ciclomorfosis (Brusca y Brusca 2005). Los cladóceros son en general filtradores no selectivos y detritívoros, existen depredadores y también parásitos. Habitan tanto en aguas continentales como marinas, en la zona pelágica, litoral y bentónica. Existen especies que viven en zonas húmedas de ambientes terrestres, incluso en ambientes subterreáneos (Forró et al. 2008).

La filogenia del grupo está muy discutida. Fryer (1987) plantea que los cladóceros no constituyen un grupo natural, mientras que otros autores afirman el carácter monofilético del grupo soportado por caracteres morfológicos (Olesen 2000) y moleculares (Spears y Abele 2000, Stenderup et al. 2006). Se reconocen cuatro órdenes: Anomopoda, Onychopoda, Ctenopoda y Haplopoda. En la región Neotropical se registran 50 géneros y en Argentina 44 de ellos están presentes (Paggi 1995).

La utilización de cladóceros en ensayos de laboratorio se debe a ciertas características que en su conjunto los hacen adecuados para desarrollar experimentos: tamaño pequeño; fácil observación, manipulación y cría; reproducción partenogenética que permite uniformidad en la respuesta; un ciclo de vida corto que permite el seguimiento completo de una cohorte; nivel trófico intermedio y sensibilidad a diversos compuestos (Castillo Morales 2004). Además, por su facilidad de cría, los cladóceros son muy utilizados como alimento vivo en la acuicultura (Das et. al. 2012). El desarrollo de un sistema de cultivo de un cladócero autóctono es un aporte importante tanto para el desarrollo de herramientas de monitoreo ambiental como para la acuicultura local.

En los protocolos estandarizados se utilizan especies que no están presentes en nuestro medio, la especie de cladóceros más utilizada y mejor conocida es $D$. magna cuya distribución está restringida al hemisferio norte (Hebert 1978). Por otro lado, el cultivo de especies exóticas como $D$. magna representa una potencial amenaza para la fauna autóctona. La introducción de fauna exótica es una de las principales causas de pérdida de biodiversidad, principalmente en ambientes acuáticos continentales (Dudgeon et al. 2006, Sala et al. 2010). Por lo tanto, resulta de interés el estudio de 
una especie residente y que pueda cumplir una función bioindicadora de ambientes regionales. Existen especies de invertebrados nativas que se utilizan como modelos para bioensayos, como es el caso del anfípodo H. curvispina Shoemaker 1942 (Mugni et al. 2010, Mugni et al. 2011, Peluso et al. 2011, Peluso et al. 2013, Rimoldi et al. 2018, Sansiñena et al. 2018) y el camarón Palaoemonetes argentinus Nobili 1901 (Graca et al. 2002, Bertrand et al. 2018). Experiencias con los cladóceros Ceriodaphnia dubia Richard 1895 (Gutierrez y Negro 2014, Regaldo et al. 2014) y D. spinulata Biraben 1917 (Alberdi et al. 1996, Ferrando 2015) muestran que es posible utilizar especies de cladóceros nativas en ensayos de laboratorio.

\section{Simocephalus vetulus (O. F. Müller 1776)}

Esta especie está presente en nuestro país (Paggi 1995) y pertenece a la familia Daphniidae (fig. 1.2). Los representantes de esta familia como Daphnia, Ceriodaphnia, entre otros, se desarrollan generalmente en la zona limnética de los ambientes acuáticos. Simocephalus vetulus, en cambio, se encuentra en la zona litoral relacionada a macrófitas, se alimenta de algas y detritos (Balayla y Moss 2003). Los organismos utilizados en este trabajo de tesis fueron inicialmente identificados por el Dr. Juan C. Paggi según el criterio de Orlova-Bienkowskaya (1998).
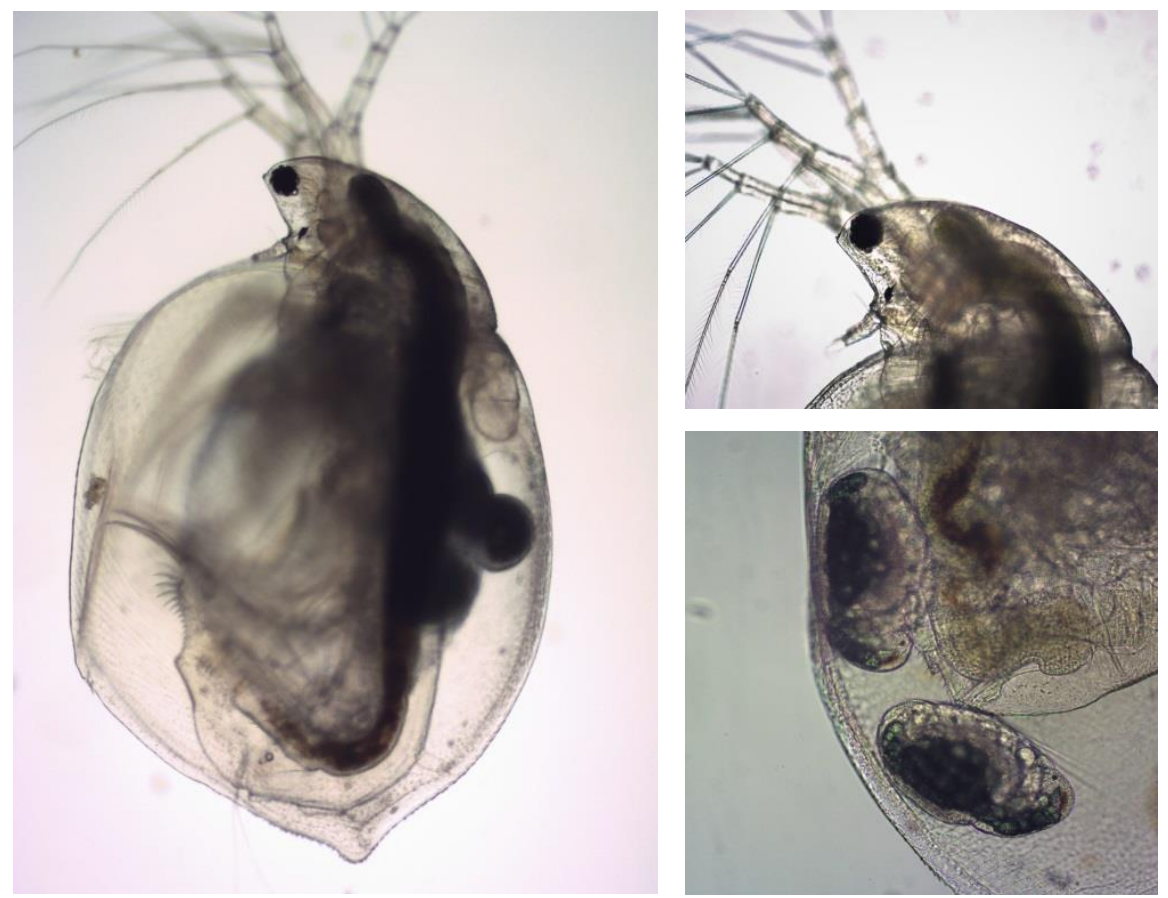

Figura 1.2. Simocephalus vetulus. Izquierda: hembra adulta con huevos en cámara de incubación (10x). Derecha: detalle de la cabeza (arriba) y de cladoceritos en cámara de incubación (abajo) (40x). Imágenes de organismos vivos bajo microscopio óptico Olympus CX33. 
Existen numerosos antecedentes de la utilización de Simocephalus vetulus en ensayos de ecotoxicología en diferentes regiones (Willis et al. 1995, Chen et al. 2004, Olvera-Hernández et al. 2004, Schroer et al. 2004, Wu et al. 2007, Reno et al. 2014), por lo que esta especie podría ser utilizada como modelo regional para ensayos de toxicidad.

En los trabajos donde se utiliza esta especie no informan un método de cultivo específico (Olvera-Hernández et al. 2004, Brito et al. 2006; van Doorslaer et al. 2007), otros sólo controlan la temperatura, entre $20-24^{\circ} \mathrm{C}$ (Perrin 1988 y 1989). Chen et al. (2004), Ferrando (2015), Lopes et al. (2011), Wu et al. (2007) controlan además el fotoperiodo, mientras que Willis et al. (1995) cultivan a $S$. vetulus bajo fotoperiodo natural e iluminación ambiental. En cuanto a la alimentación, se utilizan principalmente algas unicelulares como Chlorella vulgaris Beyerinck [Beijerinck], 1890 (Brito et al. 2006, Ferrando 2015), Ankistrodermus (Olvera-Hernández et al. 2004) Scenedesmus (Brito et al. 2006, van Doorslaer et al. 2007, Wu et al. 2007) o Raphidocelis subcapitata (=Selenastrum capicornicum) (Korshikov) Nygaard, Komárek, Kristiansen \& Skulberg, 1987 (Lopes et al. 2011). Se ha determinado que S. vetulus presenta mayores tasas de filtración cuando es alimentada con C. vulgaris que con Raphidocelis subcapitata (=Selenastrum capicornicum) (Brito et al. 2006) u otro tipo de alimento como levadura o trigo (Ferrando 2015).

Los factores ambientales influencian el crecimiento, la reproducción y la supervivencia de los seres vivos. El desarrollo depende de la temperatura y la fecundidad está relacionada a la disponibilidad de alimento (Wetzel 1981). Manteniendo la alimentación en exceso y aumentando la temperatura podrían disminuir los tiempos de maduración y de producción de neonatos y así obtener un cultivo óptimo rápidamente. Además, previo a desarrollar ensayos de toxicidad con $S$. vetulus es necesario conocer su ciclo de vida, para poder mantener un cultivo semipermanente, lograr una apropiada separación y, fundamentalmente, lograr la correcta manipulación de los individuos vivos. Incluso, la información sobre el ciclo de vida de una especie puede ayudar a comprender su papel funcional en los ambientes acuáticos.

Información como la concentración letal 50 o CL50 (concentración a la que muere el $50 \%$ de los organismos expuestos a un plaguicida) en organismos que no se distribuyen en la zona resulta de utilidad relativa en estudios en ambientes regionales. Por lo tanto, es importante desarrollar estudios con organismos representativos de la fauna local, que sean sensibles y que puedan tener una función indicadora. Los diferentes protocolos estandarizados para especies foráneas pueden ser aplicados a 
especies locales para determinar la CL50 de diferentes compuestos y así tener un estudio de base para ambientes regionales. Desarrollar estudios con especies presentes en la fauna local permite comprender de una mejor manera la estructura y la dinámica de los sistemas, permite establecer límites o niveles guía de sustancias o tóxicos utilizados en la zona y establecer pautas de manejo adecuadas según la complejidad de cada sistema.

Es importante también conocer la sensibilidad de los organismos propuestos en relación a otros utilizados en ensayos de toxicidad. El modelo de distribución de sensibilidad (Species Sensitivity Distribution, SSD) (van Straalen 2002) ajusta la variación de la sensibilidad de diferentes especies a un factor de estrés y mayormente se basa en datos de ensayos de laboratorio. Este modelo ajusta los datos de las concentraciones a las que las especies exhiben una respuesta estándar a un compuesto a una distribución normal-logarítmica linearizada, obteniéndose una curva de distribución de la sensibilidad para dicho compuesto. Permite establecer un criterio en la evaluación del riesgo de un compuesto mediante el cálculo de HC (hazardous concentrations) o concentraciones peligrosas, es decir la concentración de la sustancia que afecta a un grupo de especies (Hose 2005). Un ejemplo es la HC5 o la concentración peligrosa para el $5 \%$ de las especies o su equivalente, el nivel de protección para el 95\% (Posthuma et al. 2002). A su vez, esta herramienta permite contextualizar los datos de concentración-respuesta para un compuesto y un grupo de organismos determinados y comparar su sensibilidad (Brix et al. 2001).

\subsection{Ciclo de vida de Simocephalus vetulus}

\section{Metodología}

\section{Organismos}

La colecta inicial de los organismos se realizó en el Arroyo Destino, ubicado dentro de la Reserva de Biosfera Parque Costero del Sur (ver Capítulo 3). Los organismos fueron colectados con una red tipo D-net de $500 \mu \mathrm{m}$ de apertura de malla, fueron separados, identificados y colocados en recipientes de vidrio de $3 \mathrm{~L}$ de capacidad, con 2,5L de agua de red declorinada. El período de aclimatación fue de 60 días, bajo condiciones de fotoperiodo natural y aireación constante con temperatura controlada en $20^{\circ} \mathrm{C} \pm 1^{\circ} \mathrm{C}$ en el Bioterio del Instituto de Limnología "Dr. Raúl A. Ringuelet" (ILPLA) y fueron alimentados ad libitum con una suspensión de algas 
unicelulares (Monoraphidium sp.) cultivadas con agua de red declorinada a $25^{\circ} \mathrm{C}$, con aireación constante y agregado de solución de nitrógeno (3000 $\mu \mathrm{g} / \mathrm{L})$ y fósforo (300 $\mu \mathrm{g} / \mathrm{L}$ ), bajo un fotoperíodo 24:0 luz/oscuridad de 5000 lux de iluminación con lámparas fluorescentes de luz blanca fría.

\section{Seguimiento del ciclo de vida}

Se colocaron 20 neonatos individualmente en 20 frascos de $200 \mathrm{ml}$ con $150 \mathrm{ml}$ de agua de red declorinada y con un aireador con pipeta de vidrio. Los neonatos fueron obtenidos de hembras separadas previamente. Se dividieron en dos tratamientos: templado $\left(20^{\circ} \mathrm{C} \pm 1^{\circ} \mathrm{C}\right)$ y cálido $\left(26^{\circ} \mathrm{C} \pm 1^{\circ} \mathrm{C}\right)$. El tratamiento templado se logró controlando la temperatura por aire acondicionado a $20^{\circ} \mathrm{C}$. El tratamiento cálido se logró colocando los frascos dentro de una pecera con agua hasta la mitad de la altura de los recipientes, se colocó un calentador de 25W (Atman® Electronic Heater) y un aireador, para mantener el agua en movimiento y la temperatura uniforme en todos los frascos. La temperatura se registró diariamente para controlar que no supere el rango de variación de $\pm 1^{\circ} \mathrm{C}$ en ambos tratamientos. Durante todo el ensayo, los organismos fueron alimentados diariamente con $1 \mathrm{ml}$ de suspensión de Monoraphidium sp. ad libitum. Se realizaron observaciones diarias mediante microscopio estereoscópico (lupa) modelo Leica ${ }^{\circledR}$ EZ4, en cápsulas de petri de vidrio y con pipetas Pasteur plásticas con la punta cortada para evitar dañar a los organismos. Se registró diariamente la longitud total de cada individuo $(\mu \mathrm{m})$ utilizando una regla micrométrica calibrada en el mayor aumento (35x) (Fig. 1.3). Se registró también el número de huevos de cada individuo y el número de organismos recién nacidos.

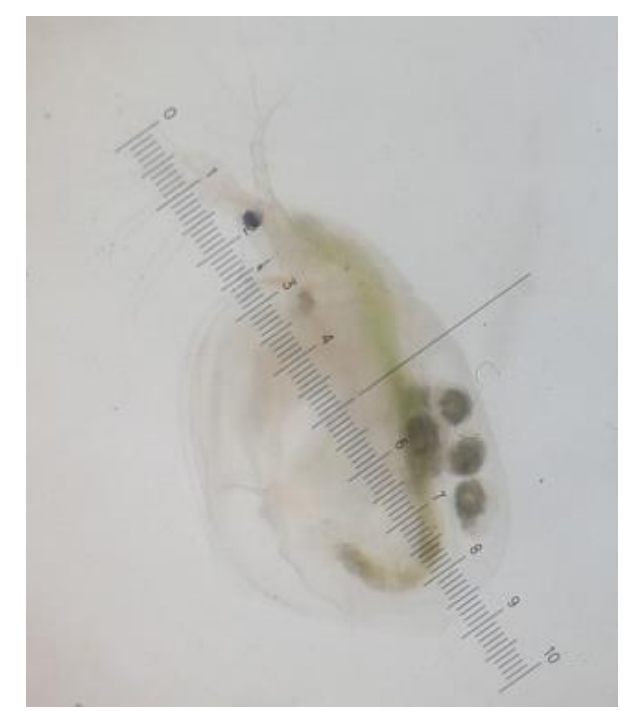

Figura 1.3. Medición del tamaño (longitud total) bajo microscopio estereoscópico (35x) y observación de huevos en cámara de incubación. 
Análisis de datos

Utilizando el número de huevos de cada hembra, el número de organismos recién nacidos y el tamaño de cada individuo, se calculó:

-Tiempo de madurez: tiempo en desarrollar huevos (días)

-Tamaño a la madurez $(\mu \mathrm{m})$

-Liberación de primer camada (días)

-Tamaño de primípara: hembra que pare por primera vez $(\mu \mathrm{m})$

-Tiempo medio de desarrollo: edad primípara - edad de madurez (días)

-Fecundidad: $\mathrm{n}^{\circ}$ de huevos por hembra por camada

$-\mathrm{N}^{\circ}$ de camadas en el ciclo de vida

-Longevidad (días).

Con los datos de longitud $(\mu \mathrm{m})$ medidos diariamente se ajustó un modelo de crecimiento mediante el modelo de von Bertalanffy (1938). La curva del modelo presenta una forma exponencial inversa, es de tipo asintótica y se basa en la siguiente fórmula:

$$
L t=L \infty\left(1-e^{-k(t-t 0)}\right)
$$

donde $L_{t}$ es la talla en un tiempo $(t), L_{\infty}$ es la talla máxima asintótica, $\mathbf{k}$ la constante de crecimiento; e es la base del logaritmo neperiano, $\mathbf{t}_{0}$ es un valor teórico que se refiere al tiempo en que los organismos presentan longitud "cero" $\left(L_{0}\right)$. Los parámetros del modelo fueron estimados a través del método de minimización de la Suma de Residuos Cuadrados (SRC), asumiendo un error de tipo aditivo y siguiendo el algoritmo de Newton (Neter et al. 1996). El procedimiento hace un nuevo cálculo del criterio de ajuste y de los parámetros del modelo. Esto se realiza por medio de iteraciones y aproximaciones sucesivas entre los valores observados y teóricos hasta encontrar el mejor ajuste (solver; Excel-Microsoft Office ${ }^{\circledR}$ ). El valor del criterio de ajuste SRC fue obtenido con la suma de las diferencias cuadráticas de los valores observados y los valores teóricos del modelo a través de la ecuación:

$$
S R C=\sum_{i=1}^{n}(\text { Lti obs }- \text { Lti esp })^{2}
$$


donde SRC es la suma de residuales cuadrados; $L_{t i}$ obs es la longitud al tiempo i para cada talla observada; $L_{t i}$ esp es la longitud al tiempo i para cada talla obtenida por el modelo. Se calcularon los intervalos de confianza al 95\%, se determinó la significancia de los parámetros del modelo con el software STATISTICA (Stat soft. Versión 7.0). Ambos modelos obtenidos se compararon entre sí mediante el método desarrollado por Aubone y Wöhler (2000) basado en la teoría de estimación de parámetros por el método de máxima verosimilitud.

La relación entre el número de huevos por hembra y el tamaño de las mismas se analizó a través de la estimación de la recta de regresión por el método de mínimos cuadrados para cada temperatura. Se realizó el ANOVA de la regresión para evaluar la significancia de la relación y posteriormente, ambas rectas se compararon estadísticamente mediante la prueba de diferencias entre dos regresiones independientes (Sokal y Rohlf 1995). Para evaluar diferencias significativas entre los tratamientos en cuanto a tiempos y talla de desarrollo de huevos y de liberación de primer camada se utilizó el test de diferencias de medias de T-Student, previamente se verificó la normalidad de los datos con el test de Shapiro-Wilk. Todos los análisis estadísticos fueron realizados con SigmaStat 3.5 a un nivel de significancia de $\alpha<0,05$.

\section{Resultados}

Se registraron diferencias significativas en cuanto al tiempo en desarrollar de huevos y en liberar la primer camada $(p<0,001)$, mientras que las diferencias de tamaño no fueron significativas ( $p=0,81$; tabla 1.1). Los organismos criados a $20^{\circ} \mathrm{C}$ presentaron mayor longevidad $(p=0,035)$. 
Tabla 1.1. Parámetros poblacionales promedio de S. vetulus y significancia estadística entre ambos tratamientos (diferencias significativas en negritas).

\begin{tabular}{lccc}
\hline Parámetros & $\mathbf{2 0 ^ { \circ } \mathbf { C }}$ & $\mathbf{2 6}^{\circ} \mathbf{C}$ & p-valor \\
\hline Neonatos: tamaño medio $(\mu \mathrm{m})$ & $573 \pm 120$ & $490 \pm 103$ & 0,12 \\
Tiempo en desarrollar huevos (días) & $9,1 \pm 0,7$ & $6,4 \pm 0,7$ & $<\mathbf{0 , 0 0 1}$ \\
Tamaño medio al desarrollar huevos $(\mu \mathrm{m})$ & $1529 \pm 153$ & $1521 \pm 142$ & 0,89 \\
Primer camada (días) & $12,9 \pm 0,8$ & $8,3 \pm 0,9$ & $<\mathbf{0 , 0 0 1}$ \\
Tamaño medio de primíparas $(\mu \mathrm{m})$ & $1721 \pm 9$ & $1705 \pm 156$ & 0,81 \\
Desarrollo embrionario (días) & $3,7 \pm 0,8$ & $2,2 \pm 0,8$ & $\mathbf{0 , 0 0 1}$ \\
Fecundidad media (huevos/hembra/camada) & $13 \pm 4,7$ & $11,6 \pm 2,6$ & 0,48 \\
No medio de Camadas & $11,5 \pm 3,3$ & $9,2 \pm 3,6$ & 0,25 \\
Longevidad media (días) & $43 \pm 15$ & $27 \pm 13,5$ & $\mathbf{0 , 0 3 5}$
\end{tabular}

Se obtuvo una curva de crecimiento para cada temperatura mediante el modelo de von Bertalanffy (Fig.1.4 y 1.5). El valor de crecimiento máximo obtenido $\left(L_{\infty}\right)$ para $20^{\circ} \mathrm{C}$ fue de $2448 \mu \mathrm{m}$ al día 50 , con los parámetros $\mathrm{k}=0,07$ y $\mathrm{t}_{0}=-2,1$; tanto $\mathrm{L}_{\infty}$ como los parámetros $\mathrm{k}$ y to fueron significativos $(\mathrm{p}<0,001$; tabla 1.2$)$. Para $26^{\circ} \mathrm{C}$, el valor de crecimiento máximo fue de $2338 \mu \mathrm{m}$ al día 34 , con $\mathrm{k}=0,11$ y $\mathrm{t}_{0}=-0,88$. $\mathrm{L}_{\infty} \mathrm{y} \mathrm{k}$ fueron significativas, no así $t_{0}$ (tabla 1.2); ambas curvas de crecimiento se ajustaron significativamente al modelo de von Bertalanffy. Al comparar los modelos entre sí mediante el método de Aubone y Wöhler no se encontraron diferencias ya que los parámetros $\left(L_{\infty}, k\right.$ y to $)$ no presentaron diferencias significativas entre sí $(p>0,05)$, por lo que Simocephalus vetulus crece de manera similar bajo ambas temperaturas. 


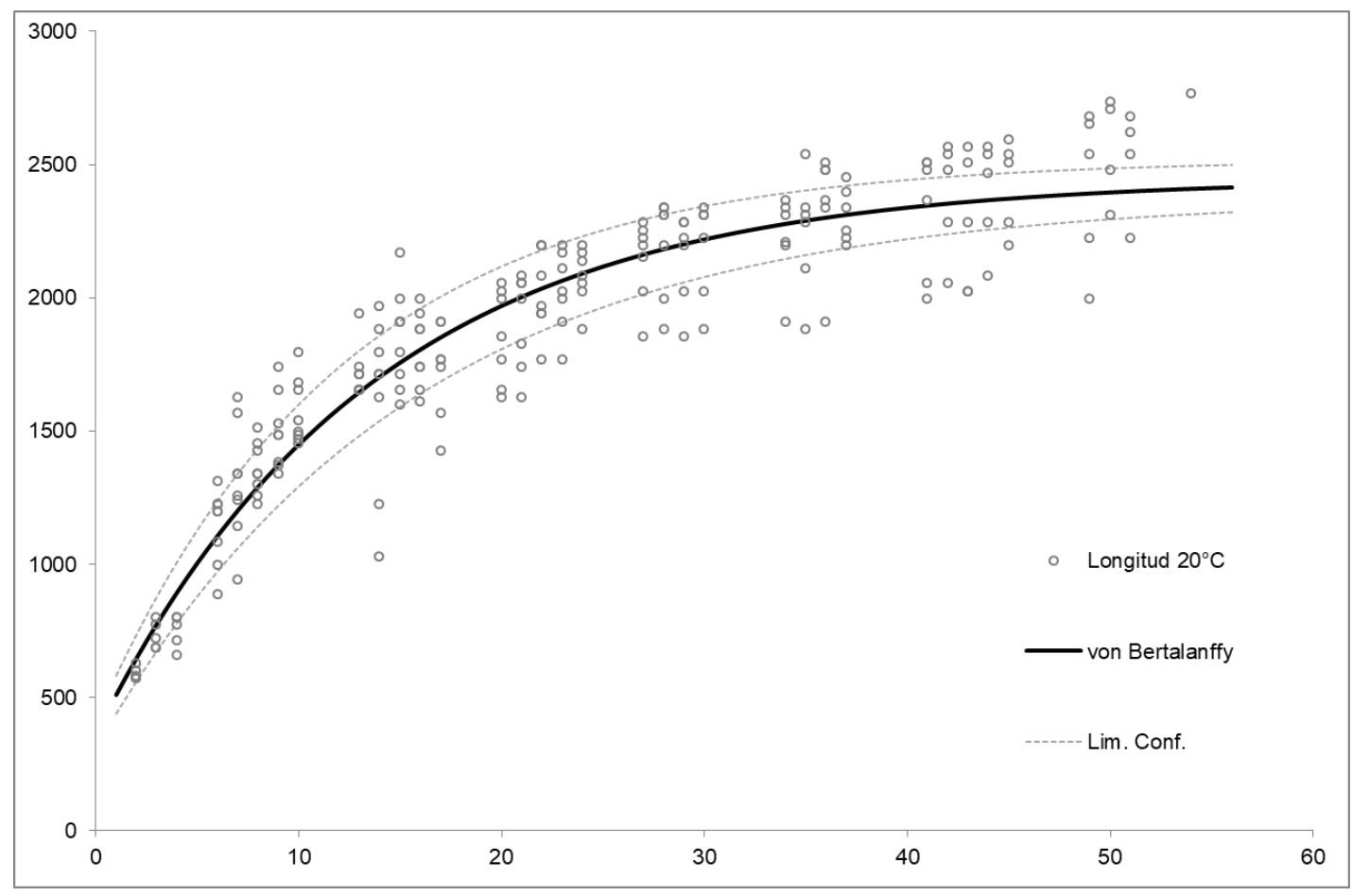

Figura 1.4. Valores medios de longitudes $(\mu \mathrm{m})$ de $S$. vetulus y modelos de crecimiento de von Bertalanffy con los respectivos límites de confianza a $20^{\circ} \mathrm{C}$.

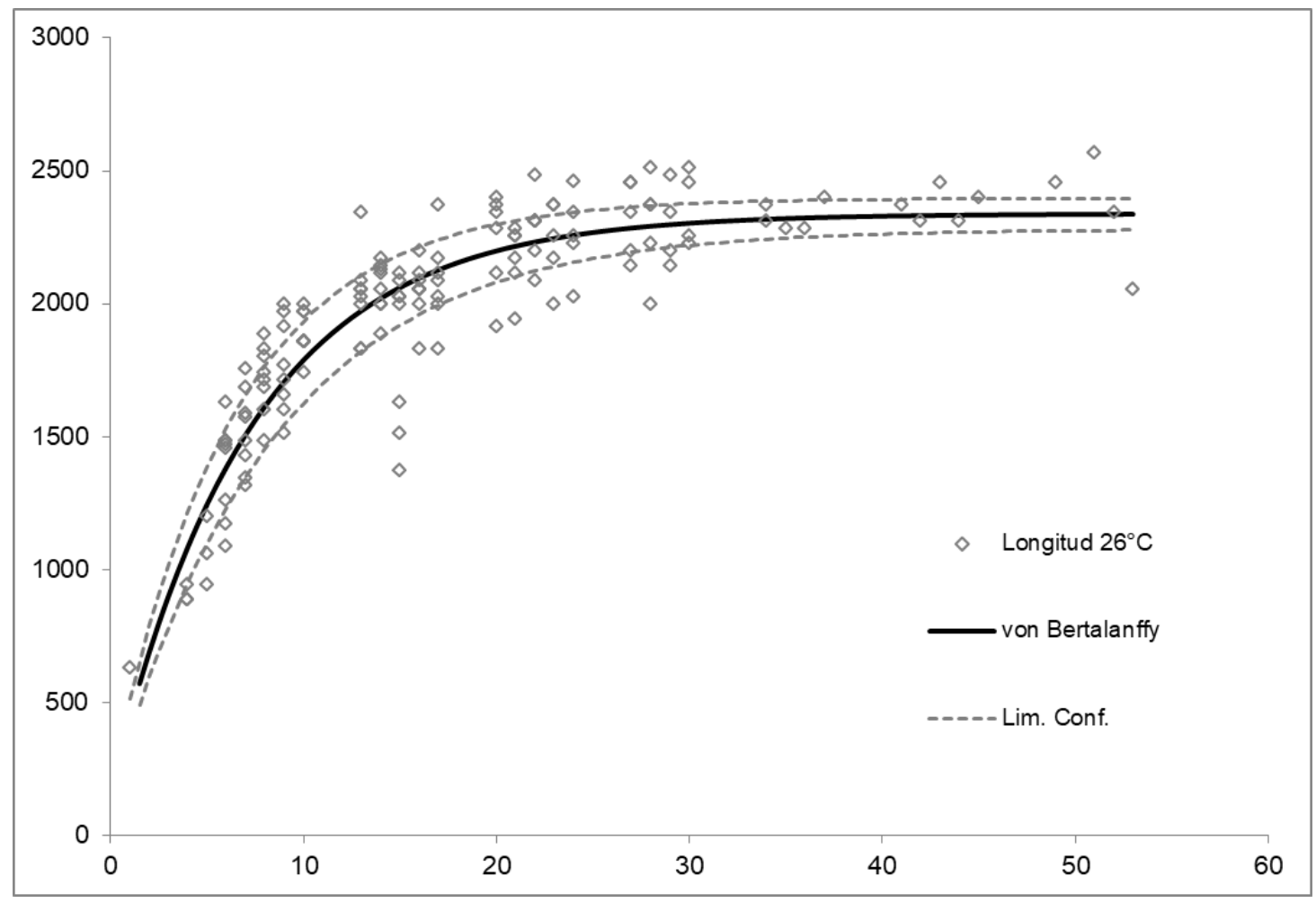

Figura 1.5. Valores medios de longitudes $(\mu \mathrm{m})$ de $S$. vetulus y modelos de crecimiento de von Bertalanffy con los respectivos límites de confianza a $26^{\circ} \mathrm{C}$. 
Tabla 1.2. Parámetros del modelo de von Bertalanffy para S. vetulus a $20^{\circ} \mathrm{C}$ y a $26^{\circ} \mathrm{C}$. En negritas se destacan los parámetros significativos.

\begin{tabular}{|c|c|c|c|c|c|c|}
\hline & Estimado & Error Standard & t-valor & p-valor & Lim. inf & Lim. Sup \\
\hline \multicolumn{7}{|l|}{$20^{\circ} \mathrm{C}$} \\
\hline $\mathrm{L}_{\infty}$ & 2448,069 & 36,01778 & 67,96834 & $<0,001$ & 2377,086 & 2519,051 \\
\hline $\mathrm{k}$ & 0,074 & 0,00467 & 15,76862 & $<0,001$ & 0,064 & 0,083 \\
\hline$t_{0}$ & $-2,164$ & 0,45171 & $-4,79165$ & $<0,001$ & $-3,055$ & $-1,274$ \\
\hline \multicolumn{7}{|l|}{$26^{\circ} \mathrm{C}$} \\
\hline $\mathrm{L}_{\infty}$ & 2338,434 & 29,28760 & 79,84382 & $<0,001$ & 2280,548 & 2396,320 \\
\hline $\mathrm{k}$ & 0,137 & 0,00947 & 14,48683 & $<0,001$ & 0,119 & 0,156 \\
\hline$t_{0}$ & $-0,541$ & 0,38069 & $-1,42186$ & 0,1572 & $-1,294$ & 0,211 \\
\hline
\end{tabular}

Con respecto a la fecundidad, la regresión lineal entre el tamaño de los organismos y el número de huevos fue significativa para ambas temperaturas $(p<0,01)$, y se obtuvieron valores de ajuste de $r=0,71$ para $26^{\circ} \mathrm{C}$ y de 0,88 para $20^{\circ} \mathrm{C}$ (Fig. 1.6). Al comparar las rectas mediante el método de comparación de pendientes no se encontraron diferencias significativas entre ellas a $20^{\circ} \mathrm{C}$ y $26^{\circ} \mathrm{C}\left(\mathrm{t}_{\text {calculado }}<\right.$ $t_{95 \% \text { g.le29). }}$.

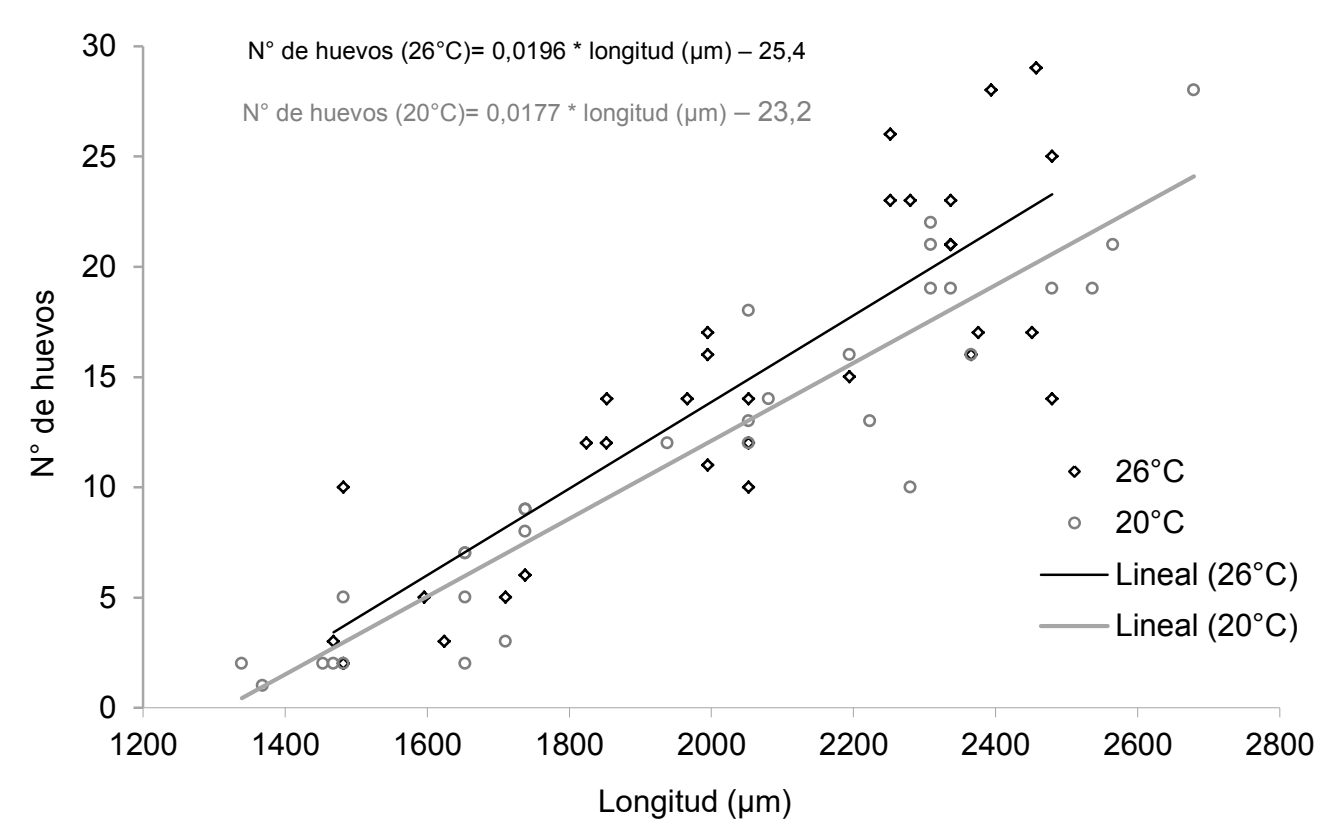

Figura 1.6. Gráficos de regresión lineal entre el tamaño $(\mu \mathrm{m})$ y número de huevos a $20^{\circ} \mathrm{C}$ y a $26^{\circ} \mathrm{C}$ y respectivas ecuaciones. 


\subsection{Cultivo de Simocephalus vetulus en laboratorio}

Cada año, se llevó a cabo la colecta de organismos para su cultivo. Se colectaron pasando una red tipo D-net de $500 \mu \mathrm{m}$ de apertura de malla por la vegetación (ver Capítulo 3). Los organismos fueron separados y los identificados como $S$. vetulus fueron colocados en frascos con agua de red declorinada en el Bioterio del ILPLA, a $22^{\circ} \mathrm{C} \pm 1^{\circ} \mathrm{C}$ y con aireación constante.

Para desarrollar el cultivo semipermanente de $S$. vetulus se evaluaron cualitativamente diferentes aspectos como el tipo de agua (agua de red o agua declorinada), la cercanía a ventanas, tipo de alimentación (algas, alimento para peces) y cuando se observó una mayor reproducción de los organismos y no se observó desarrollo de efipios se determinó que las condiciones fueron mejores. El cambio de agua se realizó una vez por semana utilizando tamices de $250 \mu \mathrm{m}$ y $350 \mu \mathrm{m}$ de apertura de malla. La alimentación se realizó dos veces por semana con una solución de Monoraphidium sp. Se midieron los parámetros fisicoquímicos oxígeno disuelto, $\mathrm{pH}$ y conductividad antes y después del recambio de agua y la alimentación y se observó una estabilidad en los valores, no variaron con los recambios de agua ni la alimentación. Los valores medios de los parámetros fisicoquímicos del cultivo fueron OD $8,96 \pm 0,54 \mu \mathrm{g} / \mathrm{L}, \mathrm{pH} 7,97 \pm 0,56$ y conductividad $678 \pm 43 \mu \mathrm{S} / \mathrm{cm}$. Posteriormente se instaló en el Instituto una Torre de Cultivo para algas, se cultivó Chlorella vulgaris en medio Bold bajo un fotoperíodo de 24:0 luz-oscuridad (luz continua) (Sathicq et al. 2017).

Se estableció entonces una metodología de mantenimiento de cultivo (ver 1.2.1.) a través del cambio de agua y alimentación periódicas, y así se logró mantener una población estable con una producción adecuada de neonatos. Se mantuvo una densidad de 12 a 15 individuos por litro, los organismos adultos fueron renovados periódicamente. Asimismo, se desarrolló una metodología de separación de neonatos (ver 1.2.2) para la realización de ensayos de toxicidad mediante la cual también se renovaba el agua y se obtenían organismos recién nacidos para la renovación del cultivo. No obstante, la población decayó sistemáticamente cada año, por lo que fue necesario renovar el cultivo anualmente. Se observó el desarrollo de efipios en uno o dos organismos por frasco de cultivo cada semana, y con una mayor frecuencia antes del decaimiento del cultivo, aunque no se determinó ningún valor extremo en los parámetros previo al decaimiento. Además, la época del decaimiento no fue estacional sino que varió a lo largo de los años, por lo que no se pudo asociar con ningún parámetro particular. 


\subsubsection{Metodología utilizada para el mantenimiento de Simocephalus vetulus}

Materiales: Bandejas plásticas (2), Tamiz de $250 \mu \mathrm{m}$ o menor

Procedimiento:

1. Colocar el tamiz de $250 \mu m$ en una de las bandejas.

2. Vaciar el contenido del frasco del cultivo sobre el tamiz, enjuagar hasta que no se observen organismos en el frasco.

3. Limpiar el frasco mecánicamente con rejilla de metal y enjuagar con agua.

4. Colocar el tamiz del revés sobre la otra bandeja plástica y enjuagar desde atrás con agua de red.

5. Colocar el contenido de la bandeja nuevamente en el frasco.

6. Completar con agua de red declorinada.

7. Alimentación: agregar solución de algas ad libitum.

\subsubsection{Metodología utilizada para la separación de neonatos $<24 \mathrm{hs}$}

Materiales: Bandejas plásticas (3), Tamiz de $250 \mu$ m o menor, Tamiz $355 \mu \mathrm{m}$ Procedimiento:

El día anterior a desarrollar el experimento:

1. Colocar el tamiz de $355 \mu \mathrm{m}$ en una bandeja (A) y el de $250 \mu \mathrm{m}$ en otra (B).

2. Vaciar el contenido del frasco del cultivo por el tamiz de $355 \mu \mathrm{m}$ sobre la bandeja A, enjuagar hasta que no se observen organismos en el frasco.

3. Colocar el tamiz de $355 \mu \mathrm{m}$ del revés sobre otra bandeja plástica (C) y enjuagar desde atrás con agua de red.

4. Colocar el contenido de la bandeja $C$ nuevamente en el frasco. Completar con agua declorinada y agregar solución de algas ad libitum $\rightarrow$ hembras adultas.

5. Pasar el contenido de la bandeja A por el tamiz de $250 \mu \mathrm{m}$ sobre bandeja B, enjuagar hasta que no queden organismos la misma. Los organismos que quedan retenidos en este tamiz corresponden a organismos para renovación del cultivo

6. Descartar el agua de la bandeja B (sin organismos).

7. Procesar todos los frascos. Al finalizar, vaciar el contenido del tamiz de $250 \mu \mathrm{m}$ sobre una bandeja libre $\rightarrow$ neonatos para renovación del cultivo.

8. El día del experimento: repetir los pasos 1-7. Ahora, los individuos que quedan retenidos en el tamiz de $250 \mu \mathrm{m}$ corresponden a neonatos $<\mathbf{2 4 h s}$. 


\subsection{Ensayos de toxicidad y sensibilidad de Simocephalus vetulus}

\subsubsection{Determinación de CL50 de plaguicidas para Simocephalus vetulus}

\section{Metodología}

Los organismos utilizados en estos ensayos corresponden al cultivo descripto en la sección anterior. Los neonatos fueron obtenidos del cultivo del Bioterio del Instituto y separados según la metodología 1.2.2. Los ensayos de toxicidad se llevaron a cabo con los insecticidas cipermetrina, lambdacialotrina y clorpirifos y con el herbicida glifosato (formulados comerciales). En la siguiente tabla se muestra la fórmula y denominación química de cada plaguicida utilizado (tabla 1.3).

Tabla 1.3. Plaguicidas utilizados en los ensayos, fórmula y nombre químico (IUPAC).

\begin{tabular}{|c|c|c|}
\hline Plaguicida & Fórmula & Nombre \\
\hline Cipermetrina & $\mathrm{C}_{22} \mathrm{H}_{19} \mathrm{Cl}_{2} \mathrm{NO}_{3}$. & $\begin{array}{c}\text { [ciano-(3-fenoxifenil)metil] 3-(2,2-dicloroetenil)- } \\
\text { 2,2dimetilciclopropano-1-carboxilato }\end{array}$ \\
\hline Lambdacialotrina & $\mathrm{C}_{23} \mathrm{H}_{19} \mathrm{ClF}_{3} \mathrm{NO}_{3}$ & $\begin{array}{l}\text { [cyano-(3-fenoxifemial)metil] (1R,3R)-3-[(Z)-2-cloro- } \\
\text { 3,3,3-trifluoroprop-1-enil]-2,2-dimetil ciclopropano-1- } \\
\text { carboxilato }\end{array}$ \\
\hline Clorpirifos & $\mathrm{C}_{9} \mathrm{H}_{11} \mathrm{Cl}_{3} \mathrm{NO}_{3} \mathrm{PS}$ & O,O-dietill O-3,5,6-tricloro-2-piridil fosforotioato \\
\hline Glifosato & $\mathrm{C}_{3} \mathrm{H}_{8} \mathrm{NO}_{5} \mathrm{P}$ & $\mathrm{N}$-fosfonometilglicina \\
\hline
\end{tabular}

Se prepararon soluciones stock con los principios activos (PA) y los formulados comerciales (FC). Como los insecticidas son compuestos hidrofóbicos, estas soluciones se prepararon en acetona de calidad analítica (J. T. Baker). El glifosato es soluble en agua, por lo que la solución stock se preparó con agua desionizada. Los ensayos con principios activos se llevaron a cabo con estándares certificados de Accustandard $\circledast$ de lambdacialotrina (99\% pureza) cipermetrina (95\% pureza) y clorpirifos (100\% pureza). Los formulados comerciales utilizados fueron los siguientes:

- Cipermetrina Galgotrin® (Chemotechnica) 25\% de ingrediente activo. Se preparó una solución stock de $100 \mathrm{ml}$ en acetona de $1 \mathrm{ppm}(1000 \mu \mathrm{g} / \mathrm{L})$. Las concentraciones nominales ensayadas fueron 0,$025 ; 0,05 ; 0,1 ; 0,2 ; 0,4 ; 0,8 ; 1,6 \mu \mathrm{g} / \mathrm{L}$. 
- Lambdacialotrina Label ${ }^{8}$ (Surcos) $5 \%$ de producto activo. Se preparó una solución stock de $100 \mathrm{ml}$ en acetona de $1 \mathrm{ppm}(1000 \mu \mathrm{g} / \mathrm{L})$. Las concentraciones nominales utilizadas en las exposiciones fueron 0,$025 ; 0,05 ; 0,1 ; 0,2 ; 0,4 ; 0,8 ; 1,6$ $\mu \mathrm{g} / \mathrm{L}$.

- Clorpirifos CLORPI® (Ciagro Santa Fe S.R.L.) 48\% de producto activo. Se preparó una solución stock de $100 \mathrm{ml}$ en acetona, obteniendo una concentración nominal de 1000ppm, que luego se diluyó en una proporción 0,1:100 para obtener la solución madre de $1 \mathrm{ppm}(1000 \mu \mathrm{g} / \mathrm{L})$. Las concentraciones nominales utilizadas en las exposiciones fueron 0,$025 ; 0,05 ; 0,1 ; 0,2 ; 0,35,0,4$ y $0,8 \mu \mathrm{g} / \mathrm{L}$.

- Glifosato Round Up Full II® (Monsanto) 66\% de producto activo. Se preparó una solución stock de $50 \mathrm{ml}$ en agua desionizada de $1324 \mathrm{mg} / \mathrm{L}$. Las concentraciones nominales utilizadas en las exposiciones fueron $1 ; 1,5 ; 2,0 ; 2,5 ; 3 ; 4$ y 5 mg/L.

Se siguió la metodología estándar propuesta por la USEPA para Daphnia magna (2002). Se expusieron 10 neonatos menores a $24 \mathrm{hs}$ a diferentes concentraciones de los plaguicidas en $30 \mathrm{ml}$ de agua declorinada en frascos de $80 \mathrm{ml}$. Se utilizaron tres réplicas para cada concentración ensayada. Se realizaron diferentes ensayos preliminares para elegir el rango de concentraciones apropiado para el desarrollo de las pruebas (range-finding test). Asimismo, se evaluó la toxicidad del solvente para verificar que no tenga un efecto tóxico en las concentraciones a ensayar. Se utilizó acetona grado plaguicida (Baker), se utilizaron 3 concentraciones (1, 10 y $100 \mu \mathrm{g} / \mathrm{L})$ y se expusieron 5 individuos por tratamiento durante 48hs, y se comprobó que no hubo mortalidad en ninguno de los tratamientos.

Las concentraciones se prepararon adicionando una cantidad de solución stock a los $30 \mathrm{ml}$ de agua declorinada hasta alcanzar la concentración requerida. Como las soluciones stock de los insecticidas se prepararon en acetona, los controles de los ensayos con estos compuestos se realizaron adicionando la cantidad de acetona que se utilizó en el tratamiento de máxima concentración. En los ensayos con glifosato el control solo contenía agua declorinada. Los organismos se consideraron muertos cuando no exhibieron movimiento luego de estimularlos con una pipeta Pasteur durante 10 segundos. Se registró la mortalidad luego de 48hs de exposición y se consideró como criterio de validez del ensayo que la mortalidad en los controles no superara el $10 \%$. Los individuos no fueron alimentados durante la exposición y los ensayos se repitieron en dos experimentos independientes. Para todos los 
experimentos las condiciones de los ensayos fueron: temperatura $20^{\circ} \mathrm{C}+/-1^{\circ} \mathrm{C}$, fotoperiodo natural, conductividad $670 \pm 35 \mu \mathrm{S} / \mathrm{cm}$, oxígeno disuelto $8,3 \pm 0,5 \mathrm{mg} / \mathrm{L}$.

\section{Análisis de Datos}

Se utilizaron los valores de mortalidad obtenidos de las exposiciones de 48hs para estimar la CL50 y los límites de confianza al 95\% mediante el método Probit. Se compararon los valores obtenidos mediante ANOVA y con el test a posteriori de Tukey. Previamente se verificaron los supuestos de normalidad y homocedasticidad. Con los valores de CL50 obtenidos se calculó un valor promedio para cada plaguicida.

\section{Resultados}

La CL50 de cipermetrina (principio activo) para S. vetulus a 48hs se determinó en un valor medio de $0,87 \pm 0,91 \mu \mathrm{g} / \mathrm{L}$, mientras que para el formulado comercial este valor fue de 0,23 $\pm 0,002 \mu \mathrm{g} / \mathrm{L}$. La CL50 promedio de lambdacialotrina (principio activo) para $S$. vetulus a $48 \mathrm{hs}$ fue de $0,21 \pm 0,01 \mu \mathrm{g} / \mathrm{L}$, mientras que para el formulado comercial fue de $0,26 \pm 0,13 \mu \mathrm{g} / \mathrm{L}$. La CL50 promedio a $48 \mathrm{hs}$ de clorpirifos (principio activo) para $S$. vetulus fue $0,38 \pm 0,42 \mu \mathrm{g} / \mathrm{L}$ mientras que para el formulado comercial este valor fue $0,08 \pm 0,015 \mu \mathrm{g} / \mathrm{L}$. La concentración letal cincuenta (CL50) promedio de glifosato fue de 1,9 $\pm 0,2 \mathrm{mg} / \mathrm{L}$ (tabla 1.4). Los resultados de los ensayos de toxicidad mostraron valores más bajos para clorpirifos formulado comercial y el valor más alto fue para glifosato.

Tabla 1.4. CL50 promedio determinadas para Simocephalus vetulus a $48 \mathrm{hs}$.

\begin{tabular}{ccc}
\hline Plaguicida & $\begin{array}{c}\text { Principio } \\
\text { Activo }\end{array}$ & $\begin{array}{c}\text { Formulado } \\
\text { Comercial }\end{array}$ \\
\hline Cipermetrina $(\mu \mathrm{g} / \mathrm{L})$ & $0,87 \pm 0,91$ & $0,23 \pm 0,002$ \\
Lambdacialotrina $(\mu \mathrm{g} / \mathrm{L})$ & $0,21 \pm 0,01$ & $0,26 \pm 0,13$ \\
Clorpirifos $(\mu \mathrm{g} / \mathrm{L})$ & $0,38 \pm 0,42$ & $0,08 \pm 0,015$ \\
Glifosato $(\mathrm{mg} / \mathrm{L})$ & - & $1,9 \pm 0,2$ \\
\hline
\end{tabular}




\subsubsection{Sensibilidad de Simocephalus vetulus en relación a otros crustáceos}

\section{Metodología}

Para evaluar la sensibilidad de $S$. vetulus en relación a otros crustáceos se calculó la curva de la distribución de sensibilidad de especies (USEPA 2015) para cada plaguicida, utilizando los valores de CL50 obtenidos en el presente trabajo y los datos de CL50 de otras especies de crustáceos (cládoceros, anfípodos, copépodos, anostracos, entre otros). Estos últimos se obtuvieron tanto de la base de datos ECOTOX (2000) como de diferentes trabajos que reportaron valores de CL50, seleccionándose los valores de experimentos realizados en condiciones similares a las del presente trabajo. Se consideraron datos tanto de formulados comerciales como de principios activos. Se incluyeron los valores para $S$. vetulus de otros autores. Para el resto de las especies, cuando se reportó más de un valor se calculó un valor medio de los registros de principios activos y otro de formulados comerciales, según correspondiera. Los nombres completos de las especies mencionadas se detallan en una lista al final del trabajo de tesis (Anexo).

Se calculó la distribución de sensibilidad (DS) ajustando los valores de diferentes especies en una distribución normal-logarítmica con el software CADDIS generator (USEPA 2015).

\section{Resultados}

Se realizó una selección de los datos considerando los valores de mortalidad en agua dulce en laboratorio a 48hs. Se seleccionaron 15 registros para cipermetrina, 10 registros para lambdacialotrina, 33 para clorpirifos y 9 para glifosato. Los valores seleccionados de la bibliografía y de bases de datos se ordenaron en tablas según cada plaguicida (tablas 1.5 a 1.8). A continuación de la tabla se muestra la curva de la distribución de la sensibilidad obtenido para cada compuesto (figuras 1.7 a 1.10).

Comparado con otros crustáceos, los valores de Simocephalus vetulus para cipermetrina principio activo y formulado comercial resultaron menores que valores para otros crustáceos como Daphnia magna, Acartia clausi y Macrobrachium lar (tabla 1.5) y la CL50 para el formulado comercial ubicó a S. vetulus dentro del $40 \%$ de especies sensibles (figura 1.7). 
Tabla 1.5. CL50 de cipermetrina de diferentes especies de crustáceos. FC= Formulado Comercial. $\mathrm{PA}=$ Principio Activo

\begin{tabular}{lccll}
\hline Especie & CL50 $(\boldsymbol{\mu g} / \mathbf{L})$ & Tipo & Referencias \\
\hline Trichodactylus borellianus & 0,02 & FC & Williner y Collins (2003) \\
Diaptomus forbesi & 0,03 & FC & Saha y Kaviraj (2008) \\
Daphnia cucullata & 0,05 & PA & Wendt-Rasch et al. (2003) \\
Hyalella curvispina & 0,065 & PA & Mugni et al. (2013a) \\
Procambarus clarkii & 0,1 & FC & Morolli et al. (2006) \\
Ceriodaphnia dubia & 0,23 & PA & Shen et al. (2012) \\
Simocephalus vetulus FC & $\mathbf{0 , 2 3}$ & FC & presente trabajo \\
Oithona similis & 0,24 & PA & Willis y Ling (2004) \\
Temora longicornis & 0,74 & PA & Willis y Ling (2004) \\
Simocephalus vetulus PA & $\mathbf{0 , 8 7}$ & PA & presente trabajo \\
Macrobrachium lar & 1,07 & FC & Bajet et al. (2012) \\
Daphnia magna PA & 2,35 & PA & Demetrio et al. (2014), Chevalier et al. (2015) \\
Acartia clausi & 2,67 & PA & Willis y Ling (2004) \\
Daphnia magna FC & 2,81 & FC & Demetrio et al. (2014) \\
\hline & & &
\end{tabular}

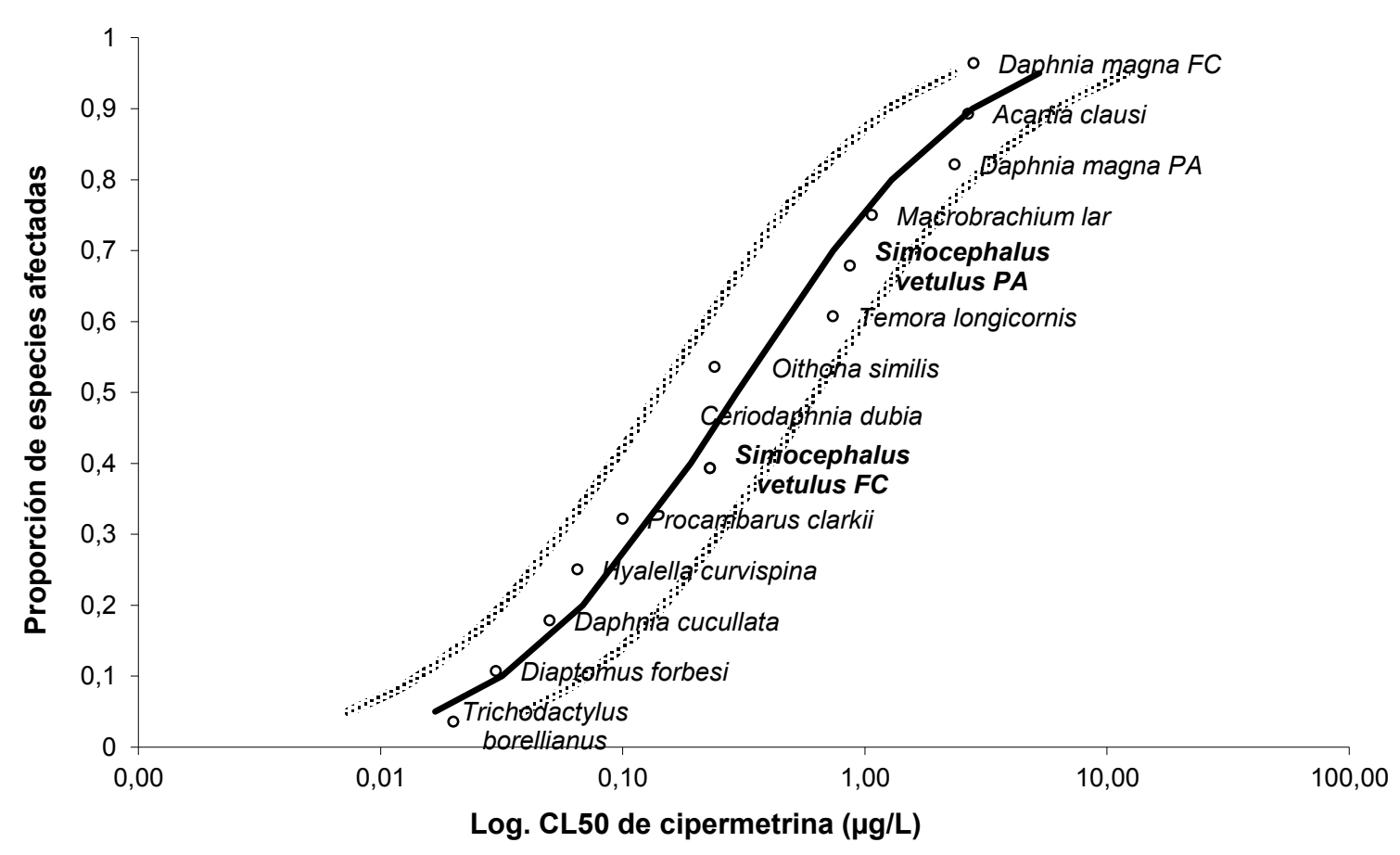

Figura 1.7. Curva de distribución de la sensibilidad de crustáceos para cipermetrina. 
Para lambdacialotrina, las CL50 para el principio activo y el formulado comercial resultaron similares, ubicando a $S$. vetulus dentro del $60 \%$ de especies sensibles a este insecticida (figura 1.8). Simocephalus vetulus mostró mayor sensibilidad que $D$. magna, D. galeata, C. dubia (tabla 1.6).

Tabla 1.6. CL50 de lambdacialotrina de diferentes especies de crustáceos. FC= Formulado Comercial. PA= Principio Activo. $\left({ }^{*}\right)$ CL50 para S. vetulus determinada por otros autores.

\begin{tabular}{lccl}
\hline Especie & CL50 $(\boldsymbol{\mu g} / \mathbf{L})$ & Tipo & Referencias \\
\hline Macrobrachium lar & 0,005 & FC & Bajet et al. (2012) \\
Streptocephalus sudanicus & 0,028 & FC & Lahr et al. (2001) \\
Gammarus pulex & 0,03 & FC & Schroer et al. (2004) \\
Proasellus coxalis & 0,08 & FC & Schroer et al. (2004) \\
Asellus aquaticus & 0,14 & FC & Schroer et al. (2004) \\
Simocephalus vetulus PA & $\mathbf{0 , 2 1}$ & PA & presente trabajo \\
Simocephalus vetulus FC & $\mathbf{0 , 2 6}$ & FC & presente trabajo \\
Ceriodaphnia dubia & 0,3 & FC & Mokry y Hoagland (1990) \\
Daphnia magna PA & 0,39 & PA & Barata et al. (2006) \\
D. galeata & 0,4 & FC & Schroer et al. (2004) \\
D. magna FC & 1,04 & FC & Mokry y Hoagland (1990) \\
S. vetulus* & 1,34 & FC & Schroer et al. (2004) \\
\hline
\end{tabular}




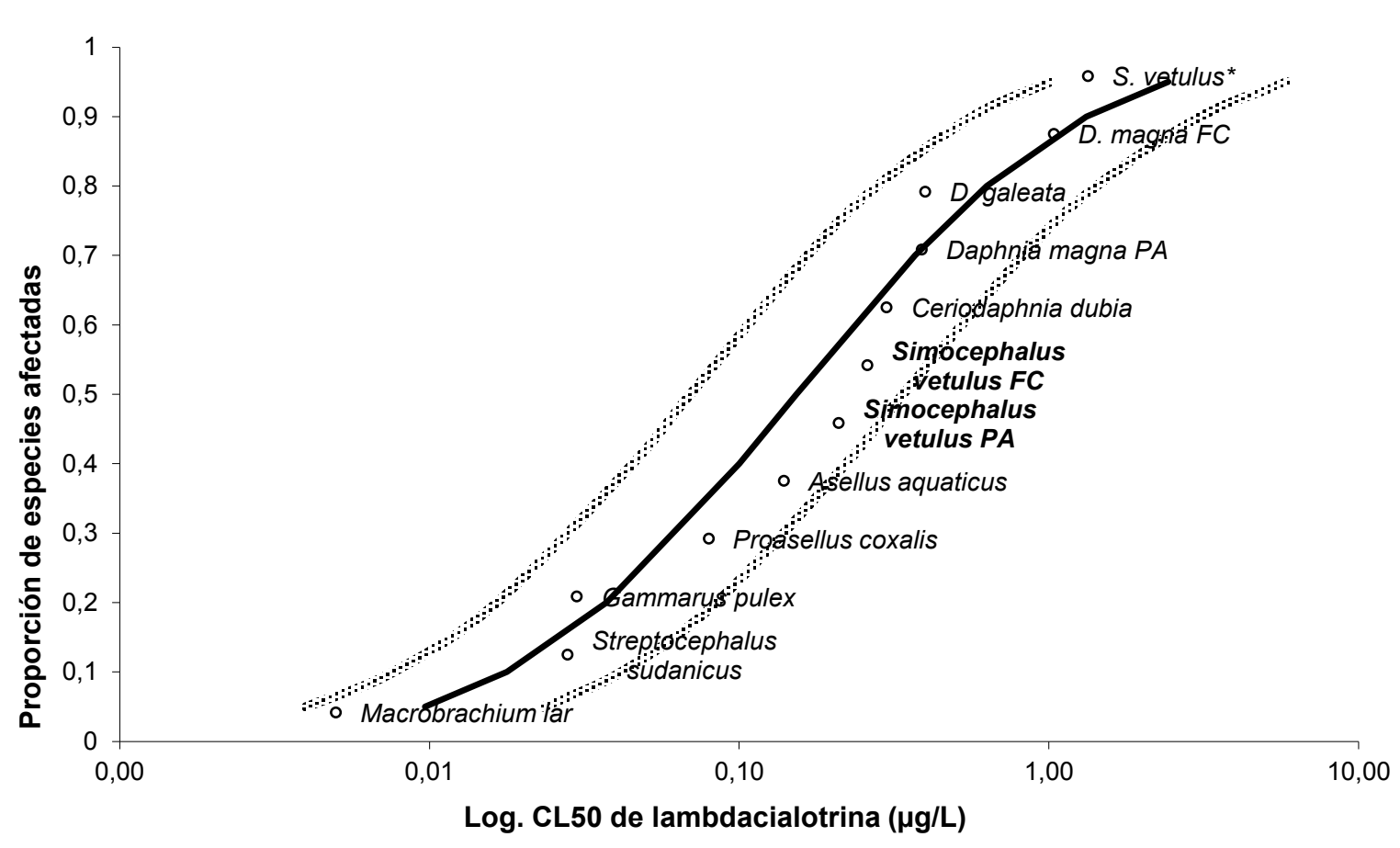

Figura 1.8. Curva de distribución de la sensibilidad de crustáceos para lambdacialotrina.

Simocephalus vetulus se ubicó dentro del $15 \%$ de especies sensibles al clorpirifos (figura 1.9) según su CL50 para el formulado comercial y presentó mayor sensibilidad que D. magna y Hyalella azteca, entre otras. La CL50 del principio activo fue también menor que la CL50 de D. magna, D. pulex y Gammarus pulex (tabla 1.7) y este valor la ubicó dentro del $40 \%$ de especies sensibles. 
Tabla 1.7. Concentraciones letales de clorpirifos de diferentes especies de crustáceos. FC= Formulado Comercial, PA= Principio Activo, NR= No reportado. $\left(^{*}\right) \mathrm{CL50}$ para S. vetulus determinada por otros autores.

\begin{tabular}{|c|c|c|c|}
\hline Especie & CL50 ( $\mu \mathrm{g} / \mathrm{L})$ & Tipo & Referencias \\
\hline Daphnia ambigua & 0,035 & FC & Harmon et al. (2003) \\
\hline Ceriodaphnia dubia & 0,055 & PA & $\begin{array}{l}\text { Bailey et al. (1997), Woods et al. (2002), } \\
\text { Harmon et al. (2003) }\end{array}$ \\
\hline Simocephalus vetulus FC & 0,08 & FC & presente trabajo \\
\hline Hyalella azteca & 0,195 & FC & Moore et al. (1998), Siefert (1987) \\
\hline D. carinata & 0,3 & PA & $\begin{array}{l}\text { Cáceres et al. (2007), Zalizniak y } \\
\text { Nugegoda (2006) }\end{array}$ \\
\hline Macrobrachium roserbergi & 0,3 & FC & Satapornvanit et al. (2009) \\
\hline Daphnia magna FC & 0,3 & $\mathrm{FC}$ & Demetrio et al. (2014) \\
\hline Gammarus pseudolimnaeus & 0,33 & $\mathrm{FC}$ & Siefert (1987) \\
\hline Simocephalus vetulus $P A$ & 0,38 & PA & presente trabajo \\
\hline H. curvispina & 0,4 & FC & Solis (2016) \\
\hline M. lar & 0,54 & FC & Bajet et al. (2012) \\
\hline D. longispina & 0,8 & FC & van Wijngaarden et al. (1993) \\
\hline S. vetulus* & 0,8 & FC & van Wijngaarden et al. (1993) \\
\hline D. pulex & 1,152 & FC & $\begin{array}{l}\text { van der Hoeven (1998), van Wijngaarden et } \\
\text { al. (1993) }\end{array}$ \\
\hline M. lanchesteri & 2,76 & PA & Tongbai et al. (2012) \\
\hline G. pulex & 3,4 & FC & $\begin{array}{l}\text { van Wijngaarden et al. (1993), Ashauer et } \\
\text { al. 2007, Rubach et al. (2011) }\end{array}$ \\
\hline Streptocephalus sudanicus & 3,48 & FC & Lahr et al. (2001) \\
\hline D. magna PA & 8,95 & PA & $\begin{array}{l}\text { Moore et al. (1998), Kersting y van } \\
\text { Wijngaarden (1992), Demetrio et al. } \\
\text { (2014), Rubach et al. (2011) y Perez et al. } \\
\text { (2015) }\end{array}$ \\
\hline P. clarkii & 23 & $\mathrm{FC}$ & Cebrián et al. (1992) \\
\hline Procambarus sp. & 34,81 & PA & Rubach et al. (2011) \\
\hline Spiralothelphusa hydrodroma & 154 & FC & SenthilKumar et al. (2007) \\
\hline Oziothelpusa & 600 & NR & Radhakrishnaiah et al. (1990) \\
\hline Neocaridina denticulata & 660 & PA & Rubach et al. (2011) \\
\hline
\end{tabular}




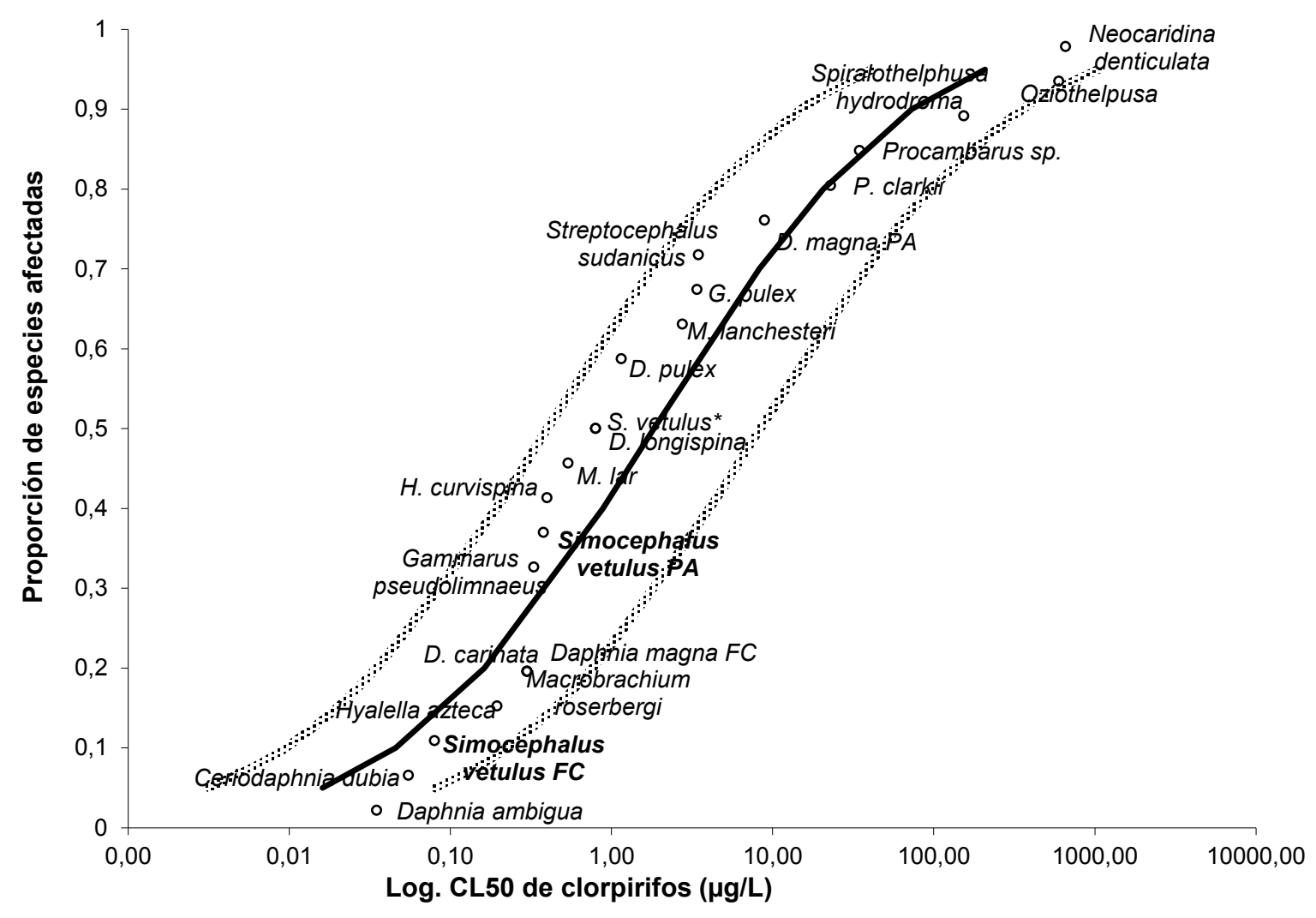

Figura 1.9. Curva de distribución de la sensibilidad de crustáceos para clorpirifos.

Para glifosato, $S$. vetulus resultó ser más sensible que $C$. dubia, $H$. curvispina y D. magna, entre otras (tabla 1.8), y se ubicó dentro del $25 \%$ de especies sensibles al herbicida (1.10).

Tabla 1.8. Concentraciones letales de glifosato para diferentes especies de crustáceos. FC= Formulado Comercial. NR= No reportado. $\left({ }^{*}\right) \mathrm{CL} 50$ de $S$. vetulus determinada por otros autores.

\begin{tabular}{cccc}
\hline Especies & Tipo & Conc. $(\mathrm{mg} / \mathrm{L})$ & Referencia \\
\hline Phyllodiaptomus annae & FC & 1,059 & Deepananda et al. (2001) \\
Hyalella azteca & FC & 1,5 & Tsui y Chu (2004) \\
Simocephalus vetulus & FC & $\mathbf{1 , 9}$ & presente trabajo \\
Ceriodaphnia dubia & FC & 5,7 & Tsui y Chu (2004) \\
Hyalella curvispina & FC & 9,9 & Mugni et al. (2015) \\
S. vetulus* & FC & 21 & Reno et al. (2014) \\
Gammarus pseudolimnaeus & NR & 42 & USEPA (1992) \\
Daphnia magna & FC & 206,5 & Henry et al. (1994), Currie et al. (2015) \\
Procambarus sp. & FC & 32650 & Abdelghani et al. (1997)
\end{tabular}




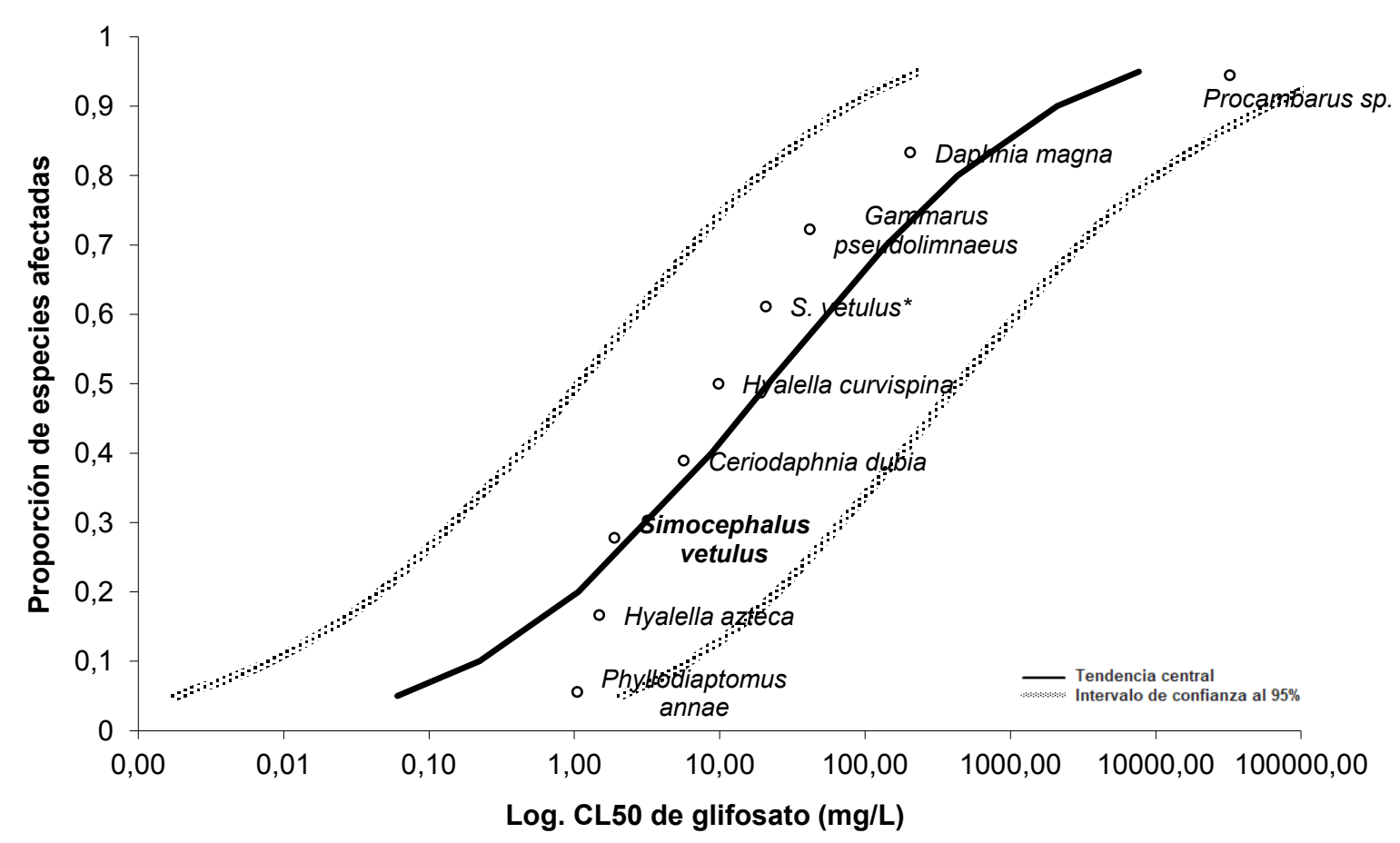

Figura 1.10. Curva de distribución de la sensibilidad de crustáceos para glifosato.

\section{Discusión}

El modelo de von Bertalanffy fue originalmente utilizado para describir el crecimiento continuo en peces. Sin embargo, es uno de los más utilizados para organismos acuáticos incluso para cladóceros, por ejemplo Moina salina Daday 1888 (Gordo et al. 1994), Macrothrix flabelligera Smirnov 1992 (Güntzel et al. 2003) y D. magna (Martínez-Jerónimo 2012). Los parámetros del modelo resultaron significativos, por lo que el modelo de von Bertalanffy fue apropiado para describir el crecimiento de S. vetulus. Sin embargo, los modelos a $20^{\circ} \mathrm{C}$ y $26^{\circ} \mathrm{C}$ no presentaron diferencias significativas entre sí, indicando que en ambas temperaturas Simocephalus vetulus crece de manera similar.

Por otro lado, los parámetros reproductivos de $S$. vetulus fueron en general más rápidos a mayores temperaturas. La mayor temperatura aumenta la velocidad metabólica, aunque existe una tendencia a reducir el tamaño de los individuos: esto fue planteado como regla general para animales endotérmicos por Bergmann (1847) y de manera similar mediante la relación superficie-volumen por Allen (1877). Uno de los patrones para ectotérmicos es la regla de la temperatura y el tamaño (temperaturesize rule, TSR) (Atkinson, 1994; Atkinson \& Sibly, 1997): los animales ectotérmicos crecen rápidamente en ambientes cálidos, pero alcanzan un tamaño adulto 
relativamente pequeño; en ambientes fríos ocurre lo opuesto, los animales retrasan la madurez pero alcanzan tamaños corporales más grandes. Perrin (1988) estudió el crecimiento en peso $(\mu \mathrm{g})$ y diferentes parámetros reproductivos de $S$. vetulus a 15,21 y $27^{\circ} \mathrm{C}$. Observó que a temperaturas altas los organismos maduraban más rápidamente y presentaron menor peso que los cultivados a bajas temperaturas. Además, determinó que la tasa de crecimiento de los neonatos disminuía a medida que disminuía la temperatura, pero que aumentaba el tamaño de los mismos. Ferrando (2015) estudió el efecto de la temperatura sobre rotíferos y determinó que a bajas temperaturas $\left(15^{\circ} \mathrm{C}\right)$ se obtuvieron individuos grandes mientras que a $25^{\circ} \mathrm{C}$ fueron un $16 \%$ menores. Por otro lado, Scheihing et al. (2011) estudiaron individuos de Limnocythere atacamae Brehm, 1935 en lagunas de diferentes temperaturas del Salar Surire en Chile durante 4 años y observaron que las poblaciones de $L$. atacamae presentaron el patrón inverso: en la laguna cálida, los individuos eran mayores que los ambientes más fríos. Los autores plantean que existen excepciones a las reglas que describen tendencias generales en el tamaño corporal. En el presente trabajo se observó una tendencia a reducir el tamaño a mayor temperatura, pero los valores no fueron significativamente diferentes.

Según Perrin et al. (1987), la limitante para el crecimiento poblacional es el tamaño de la cámara de incubación, por lo que se esperaría que los individuos más grandes presenten mayor fecundidad ya que mayor será el tamaño de la cámara y la cantidad de huevos que pueda alojar en la misma. Los resultados de la regresión entre el tamaño de los individuos y la fecundidad mostraron que la fecundidad se correlacionó positivamente con el tamaño de los organismos en ambas temperaturas. En base a los trabajos de Perrin (1988) y Ferrando (2015), se podría esperar que a mayor temperatura los individuos presenten un menor tamaño y, por lo tanto exhiban una menor fecundidad. Sin embargo, no se observaron diferencias significativas en el tamaño de los individuos y tampoco en la fecundidad, incluso la variación de la fecundidad en función del tamaño fue similar en ambos tratamientos. Podría suceder también que las temperaturas empleadas en el experimento no fueran lo suficientemente diferentes para generar diferencias significativas.

La población estudiada de Simocephalus vetulus resultó ser de tamaño relativamente grande $(>2 \mathrm{~mm})$ y la fecundidad dependió del tamaño de los individuos adultos. Los parámetros relacionados a la edad de los organismos, como la edad de desarrollar huevos, de liberar la primera camada y el desarrollo embrionario resultaron ser menores a $26^{\circ} \mathrm{C}$ (es decir, los procesos fueron más rápidos), pero los organismos vivieron menos que los criados a $20^{\circ} \mathrm{C}$. En la tabla 1.12 se presentan parámetros de 
otras especies de daphnidos comparados con los datos obtenidos en el presente trabajo. Ceriodaphnia silvestrii Daday, 1902 es una especie endémica de la región Neotropical, el límite de su distribución es el sur de Brasil; fue propuesta como organismo modelo por Fonseca y Rocha (2004) por ser una especie autóctona de pequeño tamaño que puede criarse fácilmente en laboratorio. Simocephalus vetulus presenta ciertas ventajas comparada con C. silvestrii; al ser un cladócero de mayor tamaño, es más fácil su manipulación y observación, y su alta fecundidad disminuye el requerimiento de individuos adultos para una producción óptima de neonatos. Simocephalus acutirostratus (King, 1853) es una especie mucho mayor y se desarrolla más rápidamente a temperaturas similares (Murugan y Sivaramakrishnan 1973), si bien podría ser más adecuada, es de ambientes tropicales y no se distribuye en ambientes pampeanos. La población de S. vetulus de China (Wu et al. 2007) presentó mayor fecundidad y un desarrollo madurativo más rápido que $S$. vetulus del presente trabajo, pero un tamaño mucho menor. Las poblaciones de localidades distintas pueden presentar valores diferentes de los mismos parámetros. Ebert (1991) estudió dos poblaciones de $D$. magna, provenientes de dos lagunas distantes una de la otra, al sur de Alemania. Observó que las poblaciones diferían en el tamaño de neonatos iniciales, en el tamaño de la camada y en el tamaño de las crías.

Tabla 1.9. Parámetros de otros cladóceros

\begin{tabular}{cccccc}
\hline & $\begin{array}{c}\text { Ceriodaphnia } \\
\text { silvestrii (Brasil) }\end{array}$ & $\begin{array}{c}\text { S. acutirostratus } \\
\text { (Brasil) }\end{array}$ & $\begin{array}{c}\text { S. vetulus } \\
\text { (China) }\end{array}$ & $\begin{array}{c}\text { S. vetulus } \\
\text { (Argentina) }\end{array}$ & $\begin{array}{c}\text { S. vetulus } \\
\text { (Argentina) }\end{array}$ \\
\hline $\begin{array}{c}\text { Temperatura } \\
\left({ }^{\circ} \mathbf{C}\right)\end{array}$ & 25 & $28-30$ & 20 & 20 & 26 \\
$\mathbf{N}(\mathbf{m m})$ & 0,37 & 0,65 & 0,1 & 0,5 & 0,4 \\
A máx. (mm) & 1 & 3,2 & 0,2 & 2,5 & 2,3 \\
EM (días) & $s d$ & $s d$ & 4,6 & 9 & 6 \\
TP (mm) & 0,57 & 2 & 0,1 & 1,7 & 1,7 \\
EP(días) & $s d$ & 4 & $s d$ & 12,9 & 8,3 \\
DE (días) & $s d$ & 1,9 & $s d$ & 3,7 & 2,2 \\
Fecundidad & 9 & 15 & 23 & 13 & 12 \\
(h/H/C) & 15 & 27 & $s d$ & 31 & 29 \\
F máx. & 30 & 45 & 11 & 43 & 27 \\
L (días) & & & & & \\
\hline
\end{tabular}

$\mathrm{N}$ : tamaño medio neonatos. A máx.: tamaño máximo de adultos. EM: Tiempo en desarrollar huevos - maduración.TP: tamaño primíparas. EP: edad de primíparas (días). DE: desarrollo embrionario. F: fecundidad. F máx.: fecundidad máxima. L: longevidad media; $s d=\sin$ datos. 
En cuanto a los ensayos de toxicidad, los valores de CL50 para cipermetrina y lambdacialotrina resultaron similares entre sí, mientras que para glifosato la CL50 fue de cuatro órdenes de magnitud superior. Cipermetrina y lambdacialotrina son ambos insecticidas piretroides que actúan a nivel del sistema nervioso central. Al ser la misma familia química, se puede esperar que tengan el mismo efecto, por lo tanto que su concentración letal sea similar. Asimismo, los insecticidas son utilizados para controlar insectos plaga por lo que es razonable que estos compuestos causen mortalidad en concentraciones mucho menores que los herbicidas cuya función es el control de malezas. El modo de acción del glifosato es a través de la inhibición de una enzima implicada en la síntesis de aminoácidos esenciales exclusiva de plantas superiores, pero se vincula a la generación de estrés oxidativo en células animales (Ostera et al. 2016), en base a la medición de peroxidación lipídica y la determinación de las actividades de enzimas (ej: glutatión S-transferasa). El glifosato formulado se encuentra combinado con el aditivo POEA (polioxietilenamina), surfactante utilizado para incrementar la absorción en las plantas, y que se ha demostrado que genera un aumento de la toxicidad de glifosato en invertebrados (Tsui y Chu 2004, Brausch et al. 2007, Demetrio 2012, Annet et al. 2014).

Existen varios trabajos que reportan concentraciones de plaguicidas en nuestra región. Jergentz et al. (2005) estudiaron dos arroyos en Arrecifes (provincia de Buenos Aires), una de las principales áreas de cultivo de soja de Argentina, y midieron concentraciones de cipermetrina de 0,05 a $0,7 \mu \mathrm{g} / \mathrm{L}$ en agua y de 0,13 a 0,5 $\mu \mathrm{g} / \mathrm{L}$ en agua de escorrentía superficial. Marino y Ronco (2005) determinaron 0,29 a $194 \mu \mathrm{g} / \mathrm{L}$ en agua de arroyos del sistema Pergamino-Arrecifes. Mugni et al. (2011) determinaron la presencia de cipermetrina en una concentración de $92 \mu \mathrm{g} / \mathrm{L}$ en el agua de escorrentía superficial producida por la primera lluvia con posterioridad a la aplicación de un cultivo adyacente. En estos trabajos se registran concentraciones mayores a las CL50 determinadas para $S$. vetulus en el presente trabajo $(0,23 \mu \mathrm{g} / \mathrm{L}$ para el formulado comercial), lo que sugiere que la población residente expuesta a pulsos de cipermetrina presentará efectos agudos reduciendo su densidad.

La CL50 de clorpirifos determinada en el presente trabajo fue de 0,08 $\mu \mathrm{g} / \mathrm{L}$ y los valores de clorpirifos determinados en arroyos pampeanos fueron similares a los de cipermetrina. Jergentz et al. (2005) midieron 0,45 $\mu \mathrm{g} / \mathrm{L}$ en Arrecifes, mientras que Marino y Ronco (2005) midieron de 0,2 - 10,8 $\mathrm{gg} / \mathrm{L}$. En el trabajo de Mugni et al. (2011) determinaron $0,11 \mu \mathrm{g} / \mathrm{L}$ en el agua de inundación con posterioridad a la aplicación. De la misma manera, la concentración en el ambiente registrada es similar o mayor a la concentración letal determinada por lo que se espera un efecto agudo sobre la población por aumento de la mortalidad. 
Las medidas de lambdacialotrina en arroyos pampeanos son escasas y fueron medidas en sedimento. Hunt et al. (2016) determinaron valores de entre 0,42 y $6 \mathrm{ng} / \mathrm{g}$ en agua de un arroyo pampeano en Arrecifes en un sitio de intensa actividad agrícola, mientras que en el Cinturón Hortícola, Mac Louglin et al. (2017) midieron lambdacialotrina en el rango de 1,48 a 649 ng/g y Sansiñena et al. (2018) midieron 48,6 a $265 \mu \mathrm{g} / \mathrm{kg}$ en el Arroyo Carnaval. La presencia de lambdacialotrina en los sedimentos sugiere aportes desde la cuenca y, por consiguiente, la eventual ocurrencia de eventos de toxicidad para los organismos expuestos.

Peruzzo et al. (2008) determinaron valores de glifosato en arroyos superficiales del sistema Pergamino-Arrecifes en concentraciones de 0,1 a $0,7 \mathrm{mg} / \mathrm{L}$ en agua, y Aparicio et al. (2013) registraron un rango de 0,5-7,6 $\mu \mathrm{g} / \mathrm{L}$ de glifosato en agua en arroyos del SE de la Provincia de Buenos Aires. Ronco et al. (2016) estudiaron tributarios del río Paraná y detectaron valores de alrededor de los 0,6 $\mu \mathrm{g} / \mathrm{L}$. En el Cinturón Hortícola el uso de glifosato es limitado, sin embargo, Mac Louglin et al. (2017) determinaron valores de 11 a $404 \mathrm{ng} / \mathrm{g}$ en sedimentos del arroyo Carnaval. Si bien las concentraciones determinadas en agua son menores a la CL50 de glifosato para S. vetulus, podrían existir efectos subletales de este herbicida en parámetros del ciclo de vida, como por ejemplo, la reducción en el crecimiento y la reproducción de Daphnia magna demostrado por Cuhra et al. (2013) en exposiciones menores a 1mg/L de Round $U p \circledR$. Las concentraciones utilizadas en dicho trabajo $(0,05 ; 0,15 ; 0,45 \mathrm{mg} / \mathrm{L})$ son valores ambientalmente realistas en arroyos pampeanos.

Simocephalus vetulus se encontró entre el $50 \%$ y $60 \%$ de especies sensibles para cipermetrina y para lambdacialotrina, y resultó más sensible que el 80 y $90 \%$ de los crustáceos para clorpirifos y glifosato, respectivamente. Comparado con otros cladóceros, la población de $S$. vetulus estudiada en el presente trabajo resultó ser más sensible que Ceriodaphnia dubia y Daphnia magna a cipermetrina. De los organismos más sensibles que S. vetulus, Diaptomus forbesi, D. cucullata y Procambarus clarkii o "cangrejo de río americano" son especies de Estados Unidos o Europa, no están presentes en nuestro país, sólo el cangrejo Trichodactyllus borellianus y el anfípodo Hyalella curvispina se encuentran en Argentina.

En cuanto a clorpirifos, $S$. vetulus fue de las más sensibles, entre ellas, $D$. ambigua y $C$. dubia y resultó ser más sensible que $D$. magna, la especie más utilizada. D. ambigua se encuentra en el SO de Estados Unidos y C. dubia se distribuye en nuestro país, sin embargo, es de menor tamaño que $S$. vetulus y habita principalmente ambientes lénticos o ríos de gran envergadura, como el Río Paraná. 
Simocephalus vetulus presentó el valor más bajo de CL50 para lambdacialotrina y glifosato de los cladóceros comparados, por lo que se lo puede considerar como la especie de cladócero más sensible para ambos compuestos. Los organismos con mayor sensibilidad a lambdacialotrina que $S$. vetulus fueron el camarón de agua dulce Macrobrachium lar, el anostraco Streptocephalus sudanicus, el anfípodo G. pulex y los isópodos Asellus aquaticus y Proasellus coxalis, ninguno presente en nuestra región. De igual manera, los más sensibles a glifosato fueron el copépodo calanoideo Phyllodiaptomus annae y el anfípodo $H$. azteca, ninguno de ellos presentes en Argentina. Según estos registros, se puede concluir que $S$. vetulus es la especie de distribución local más sensible a lambdacialotrina y glifosato.

Se ha demostrado que la sensibilidad a los compuestos varía entre poblaciones diferentes de la misma especie (Anguiano et al. 2008). Van Wijngaarden et al. (1993) determinaron la CL50 de clorpirifos para Gammarus pulex en 0,08 $\mu \mathrm{g} / \mathrm{L}$ mientras que Rubach et al. (2011) determinaron un valor de 0,43 $\mu \mathrm{g} / \mathrm{L}$ y Ashauer et al. (2007) un valor de 9,39 $\mu \mathrm{g} / \mathrm{L}$ para la misma especie. Demetrio et al. (2014) determinaron la CL50 de $D$. magna para cipermetrina en $0,3 \mu \mathrm{g} / \mathrm{L}$ mientras que Moore et al. (1998) determinaron un valor de $0,6 \mu \mathrm{g} / \mathrm{L}$. En el presente trabajo de tesis, las CL50 determinadas para $S$. vetulus resultaron diferentes a las determinadas anteriormente por otros autores para la misma especie, como los valores informados por Reno et al. (2014) para glifosato, van Wijngaarden et al. (1993) para clorpirifos y Schroer et al. (2004) para lambdacialotrina. Esto resalta la importancia de realizar estudios a nivel local ya que la misma especie puede presentar diferentes valores de CL50 en distintas regiones.

Considerando los resultados obtenidos del ciclo de vida y en relación a otros organismos, Simocephalus vetulus presenta características apropiadas para considerarla como potencial especie modelo de ensayos de laboratorio para ambientes pampeanos. Es necesario profundizar el estudio de los requerimientos de la especie para desarrollar un cultivo estandarizado y asegurar una provisión adecuada y constante de individuos en laboratorio.

Las concentraciones letales de los plaguicidas más utilizados en nuestro medio determinadas en el presente trabajo junto al análisis de sensibilidad comparada muestran que $S$. vetulus se encuentra entre los organismos más sensibles de distribución local, por lo que resulta de gran utilidad en la realización de ensayos de toxicidad y como modelo para la evaluación de riesgo en los ambientes pampeanos.

El análisis de las concentraciones de plaguicidas registradas en la bibliografía, en ocasiones mayores a las concentraciones letales, sugieren la ocurrencia de efectos 
letales por exposición estos compuestos en las poblaciones residentes de $S$. vetulus en los arroyos. Una población sometida a sólo un pulso de toxicidad podría recuperar su densidad por repoblamiento. No obstante, si las poblaciones están expuestas a reiterados pulsos de toxicidad, se podría esperar su declinación en el tiempo. En el próximo capítulo se estudia el efecto de la exposición a repetidos pulsos de toxicidad en S. vetulus. 


\section{CAPÍTULO 2:}

Efectos sub-letales de la exposición a pulsos de toxicidad de plaguicidas sobre Simocephalus vetulus 


\section{CAPÍTULO 2: Efectos sub-letales de la exposición a pulsos de toxicidad de plaguicidas sobre Simocephalus vetulus}

\section{Introducción}

Existen numerosos antecedentes sobre los efectos de plaguicidas sobre organismos no-blanco. Los plaguicidas tienen efectos letales y sub-letales que se pueden determinar en distintos puntos finales a diferentes niveles ecológicos. Pueden mencionarse, entre otros, estudios realizados a nivel de organismo en crustáceos como por ejemplo: efectos en el consumo de oxígeno y contenido de carbohidratos y lípidos (Verslycke et al. 2004), comportamiento natatorio (Roast et al. 2000, Oliveira et al. 2012), eficiencia alimentaria (Gliwicz y Sieniawska 1986, Bengtsson et al. 2004), en el ciclo diario de movimiento del zooplancton (Gutierrez et al. 2012) y el crecimiento (Hanazato 2001). Christensen et al. (2005) estudiaron la eficiencia de la alimentación y la habilidad de nado de Daphnia magna expuesta a concentraciones crónicas de cipermetrina, registrando efectos negativos a una concentración de 0,05 $\mu \mathrm{g} / \mathrm{L}$ que representa un $5 \%$ de su CL50.

A nivel de población se pueden estudiar los procesos de crecimiento poblacional a través de la tasa de crecimiento "r" (Forbes y Calow 1999, Stark y Banks 2003) y los procesos de reproducción, que permiten hacer estimaciones sobre la dinámica poblacional. Muchos de los plaguicidas utilizados en cultivos actúan como disruptores endócrinos y afectan al proceso reproductivo. Se ha determinado la disminución de la fecundidad en Daphnia expuesta a toxafeno (Kashian y Dodson 2002), clorpirifos (Zalizniak y Nugegoda 2006) y a deltametrina (Toumi et al. 2013).

Los bioensayos bajo exposiciones continuas (manteniendo constante la concentración de exposición) permiten obtener información acerca de los efectos de los plaguicidas en los organismos, aunque este tipo de exposición no sea lo que comúnmente suceda en la naturaleza. Una de las principales vías de ingreso de los plaguicidas a los cuerpos de agua es la escorrentía superficial (Schulz 2001, Jergentz et al. 2005), este proceso depende de la frecuencia de lluvias y de las aplicaciones en los cultivos, eventos que son episódicos y no ocurren constantemente.

Por otro lado, la concentración de los plaguicidas en los arroyos decae por diferentes procesos, como dilución, transporte aguas abajo, fotólisis o la adsorción por los sedimentos. Por ejemplo la cipermetrina, que presenta un coeficiente de reparto 
octanol-agua log Kow de 6,3 es adsorbida a los sedimentos rápidamente, incluso a las pocas horas de la aplicación (Jones 1995, Maund et al. 2002). Sumado a esto, la disminución de la concentración está acompañada por un decaimiento de la toxicidad. Mugni et al. (2010) estudiaron la mortalidad de Hyalella curvispina luego de una aplicación experimental de cipermetrina $(0,55 \mu \mathrm{g} / \mathrm{L})$ y clorpirifos $(0,12 \mu \mathrm{g} / \mathrm{L})$ en una charca poco profunda a las 24,48 y $72 \mathrm{hs}$ posteriores a la exposición, y con agua de la charca tomada a los $30 \mathrm{~min}, 4,24$ y $48 \mathrm{hs}$ posteriores a la aplicación. Observaron que la mortalidad tanto in situ como en laboratorio disminuyó de $100 \%$ a las 24 hs luego de la exposición a menos del 20\% al tercer día. Según Knauer et al. (2017) esta disminución de la toxicidad puede deberse a la interacción con los sólidos suspendidos o la materia orgánica. Por lo tanto, la exposición a plaguicidas de la fauna residente en un arroyo será episódica y durante un tiempo corto.

Como fue expresado en el capítulo anterior, en los estudios de monitoreo o determinación puntual de plaguicidas, las concentraciones que se determinan en los cuerpos de agua en general son bajas, mientras que las concentraciones altas son aisladas. Los organismos pueden estar expuestos a altas concentraciones (mayores o iguales a una CL50) por un corto periodo de tiempo, resultando en pulsos de toxicidad (Richards y Bakers 1993, Naddy et al. 2000).

Resulta de interés entonces desarrollar experiencias que simulen el comportamiento de los plaguicidas en el ambiente, por lo tanto, una exposición a pulsos repetidos de corta duración puede ser un diseño apropiado para evaluar los efectos de los plaguicidas en la fauna de los arroyos.

\subsection{Efectos a nivel organísmico}

\section{Diseño Experimental I: supervivencia, crecimiento y reproducción.}

\section{Metodología}

Neonatos menores de 24 hs fueron expuestos a pulsos de toxicidad de $60 \mathrm{~min}$ lambdacialotrina formulado comercial Label囚. Pasado el tiempo de exposición, los organismos fueron colocados individualmente en frascos con medio de cultivo sin plaguicida y se registraron diferentes parámetros diariamente durante 15 días. 
Se utilizó la solución stock descripta en el Capítulo 1. Se utilizaron tres concentraciones y un control. Las concentraciones utilizadas se establecieron a partir de un porcentaje de la CL50 obtenida para $S$. vetulus (aproximadamente 0,2 $\mu \mathrm{g} / \mathrm{L}$ ): el tratamiento 1 (T1) fue de $0,05 \mu \mathrm{g} / \mathrm{L}$ (correspondiendo a un $25 \%$ de CL50); el tratamiento 2 (T2) fue $0,2 \mu \mathrm{g} / \mathrm{L}$ (equivalente a la CL50); y el tratamiento 3 (T3) fue 1 $\mu \mathrm{g} / \mathrm{L}$ (500\% la CL50). La exposición de los organismos se realizó en frascos de $80 \mathrm{ml}$ con $30 \mathrm{ml}$ de agua declorinada adicionando un volumen de solución stock hasta alcanzar la concentración de exposición del tratamiento. Los controles se realizaron de igual manera que en las determinaciones de la CL50 (ver Capítulo 1). Se utilizaron 10 organismos individualizados por tratamiento, por lo que cada tratamiento contó con 10 réplicas (un individuo por frasco). Luego de la exposición, los organismos fueron colocados en frascos de $80 \mathrm{ml}$ con $30 \mathrm{ml}$ de medio de cultivo, que se preparó con agua de red declorinada utilizando como alimento una suspensión de Chlorella vulgaris en una concentración de $0,5 \times 10^{6} \mathrm{cél} / \mathrm{ml}$.

Se realizó el seguimiento diario de los individuos registrando la supervivencia, la longitud $(\mu \mathrm{m})$, el número de mudas y número de neonatos nacidos. La exposición inicial (día 0) se realizó en todos los individuos del mismo tratamiento en el mismo frasco. Se repitió la exposición de manera individual luego de la liberación de neonatos, en total los organismos fueron expuestos a 3 pulsos de lambdacialotrina en cada tratamiento a una misma concentración. Las condiciones del experimento fueron temperatura $22^{\circ} \mathrm{C} \pm 1^{\circ} \mathrm{C}$ y fotoperiodo natural (verano). La renovación del medio se realizó diariamente luego del recuento para asegurar la disponibilidad de oxígeno y de alimento.

\section{Análisis de datos}

Se determinaron parámetros como puntos finales: la longevidad $\left(^{\circ}\right.$ de días vividos al finalizar el experimento), la longitud al final del experimento, el número total de mudas por individuo, el número de huevos por hembra por día (tamaño de camada) y los días hasta liberar la primera camada.

Para evaluar diferencias entre el control y los tratamientos se realizó un ANOVA de una vía con cada uno de los parámetros determinados y se utilizó el test de Tukey para evaluar diferencias entre los tratamientos. Previamente se verificaron los supuestos de normalidad con el test de Shapiro Wilk y homocedasticidad con el test de Levene. Cuando lo datos no se ajustaron a una distribución normal o no presentaron homocedasticidad, se aplicó el análisis de Kruskall Wallis y el test a posteriori de 
Dunn's. Todos los análisis se realizaron con Sigma Stat 5.5 y se analizaron con un nivel de significancia de $\alpha=0,05$.

\section{Resultados}

En total, los organismos fueron expuestos a 3 pulsos de lambdacialotrina a lo largo de los 15 días del experimento, aproximadamente cada 7 días. No se observó mortalidad durante ni inmediatamente después de la exposición. No obstante, algunos organismos del tratamiento de mayor concentración permanecieron inmóviles en el fondo del recipiente inmediatamente después de la exposición, lo cual fue revertido unas pocas horas después de haber sido transvasados a agua limpia. Al finalizar el experimento, el control alcanzó una mortalidad del 30\%, el tratamiento 1 presentó un $40 \%$, el tratamiento 2 un $70 \%$ y el tratamiento 3 un $90 \%$ de mortalidad.

Los parámetros determinados al final del experimento (día 15) se muestran en la tabla 2.1. La longevidad presentó diferencias significativas $(p=0,037)$ siendo el tratamiento 3 diferente del control $(p<0,05)$. No se observaron diferencias significativas en el tamaño final de los individuos $(p=0,09)$ pero sí en el número total de mudas $(p=0,022)$. Los tratamientos T1 y T2 no presentaron diferencias con el control y T3 fue diferente de T1 $(p<0,05)$.

Tabla 2.1. Parámetros finales promedio de $S$. vetulus expuesto a pulsos de lambdacialotrina durante $60 \mathrm{~min}$. En negritas se muestras los valores significativos $(p<0,05)$.

\begin{tabular}{cccccc}
\hline & $\begin{array}{c}\text { Longevidad } \\
\text { media (días) }\end{array}$ & $\begin{array}{c}\text { Tamaño } \\
\text { medio }(\mu \mathrm{m})\end{array}$ & $\begin{array}{c}\text { Mudas } \\
\text { promedio }\end{array}$ & $\begin{array}{c}\mathrm{N}^{\circ} \text { medio de } \\
\text { huevos/hembra }\end{array}$ & $\begin{array}{c}\text { 1er camada } \\
\text { (días promedio) }\end{array}$ \\
\hline Control & $11,8 \pm 4,9$ & $1229 \pm 108$ & $4,5 \pm 1,8$ & $2,25 \pm 0,5$ & $11,5 \pm 2,4$ \\
T1 & $10,4 \pm 5,8$ & $1431 \pm 300$ & $6 \pm 1,5$ & $2,6 \pm 1,5$ & $11 \pm 2,6$ \\
T2 & $9,6 \pm 4,8$ & $1140 \pm 57$ & $3,6 \pm 2,3$ & $2 \pm 0$ & $8,25 \pm 1,3$ \\
T3 & $5 \pm 3,8$ & $1140 \pm 0$ & $2,2 \pm 1,1$ & $2 \pm 0$ & $9 \pm 0$ \\
\hline p-valor & $\mathbf{0 , 0 3 7}$ & 0,09 & $\mathbf{0 , 0 2 2}$ & 0,897 & 0,11 \\
\hline
\end{tabular}

Para una correcta interpretación de los resultados, se calculó el número de mudas en función de la longevidad, es decir, cuánto mudaron los organismos en relación al tiempo de vida. Esta proporción se sometió al ANOVA de una vía y no se 
encontraron diferencias significativas entre el control y tratamientos $(p=0,5)$, por lo que la diferencia en el número de mudas totales podría deberse a la reducción en la supervivencia.

Con respecto a los parámetros reproductivos, los individuos que se reprodujeron lo hicieron dos veces en los 15 días del experimento. En el Control sólo se reprodujo el $40 \%$ de los individuos, en T1 se reprodujo el $50 \%$, en T2 hubo un $40 \%$ de individuos reproductivos, y en el tratamiento 3 , el de mayor mortalidad, el único organismo sobreviviente al día 15 se reprodujo dos veces (10\% del total del tratamiento). Sin embargo, no se observaron diferencias significativas en cuanto al número de huevos de la primer camada ni en los días en liberarla $(p>0,05)$.

\section{Diseño experimental II: alimentación.}

\section{Metodología}

Con el objetivo de evaluar efectos de pulsos de toxicidad subletal a corto plazo, se estudió la inhibición de la alimentación en individuos de $S$. vetulus expuestos a un pulso de 90 min de cipermetrina formulado comercial Galgotrin®. Pasado el tiempo de exposición, los organismos fueron colocados en frascos con medio de cultivo. Se determinó la concentración de algas no ingeridas a las $3 \mathrm{hs}$ y a las $24 \mathrm{hs}$ posteriores a la exposición y la supervivencia de los organismos al final del experimento.

Al igual que el experimento anterior, se utilizaron las concentraciones stock descriptas en el capítulo 1 y las concentraciones utilizadas se establecieron a partir de un porcentaje de la CL50 (aproximadamente 0,2 $\mathrm{gg} / \mathrm{L}$ ): el tratamiento 1 (T1) fue de $0,02 \mu \mathrm{g} / \mathrm{L}$; el tratamiento 2 (T2) fue $0,2 \mu \mathrm{g} / \mathrm{L}$; y el tratamiento 3 (T3) fue $1 \mu \mathrm{g} / \mathrm{L}$, correspondiendo a $10 \%, 100 \%$ y $500 \%$ de la CL50. Se utilizaron 5 organismos de $S$. vetulus de 5 días de edad y 4 réplicas por tratamiento $(\mathrm{N}=20)$. La exposición se realizó en frascos de $80 \mathrm{ml}$ con $30 \mathrm{ml}$ de agua de red declorinada adicionando un volumen de solución stock hasta alcanzar la concentración de exposición del tratamiento. Los controles se realizaron de igual manera que en las determinaciones de la CL50.

El medio de cultivo utilizado corresponde al del experimento anterior. Se preparó $1 \mathrm{~L}$ de medio, se tomaron 3 alícuotas para medir la concentración de algas, y luego se colocaron $30 \mathrm{ml}$ en cada frasco, por lo que se asumió la misma concentración inicial para todos los tratamientos. Se adicionó un tratamiento sin organismos, sólo con 
medio de cultivo (sólo alga, SA) para evaluar la concentración de algas al final del experimento.

Pasado el tiempo de exposición (90 min), los organismos fueron colocados en los frascos con medio de cultivo, y se colocaron en agitación continua en un agitador Vicking Shaker Pro ${ }^{\circledR}$ dispuestos al azar por $24 \mathrm{hs}$. La concentración de algas se determinó tomando una alícuota de $1 \mathrm{ml}$ del sobrenadante de cada frasco a las 3 y 24 hs. El recuento se realizó mediante cámara de Neubauer bajo microscopio óptico Olympus ${ }^{\circledR}$ CX33. Se registró la supervivencia de los organismos a la 3 y a las $24 \mathrm{hs}$ posteriores a la exposición.

\section{Análisis de datos}

Las concentraciones de algas determinadas a las $3 \mathrm{hs}$ y a las $24 \mathrm{hs}$ luego de la exposición se analizaron mediante un análisis de Kruskal Wallis seguido de un test de Dunnet para evaluar diferencias con el Control. Estos análisis se realizaron con Sigma Stat 5.5 y se analizaron con un nivel de significancia de $\alpha=0,05$.

\section{Resultados}

La concentración inicial de algas fue determinada en $435.000 \pm 42.000$ cél $/ \mathrm{ml}$. No se registró la mortalidad en ninguno de los tratamientos. La supervivencia de los organismos fue del $100 \%$ en todos los tratamientos. A las 3 hs posteriores a la exposición, se observaron diferencias significativas entre los tratamientos $(p=0,002)$; el control registró una concentración media de algas significativamente menor al tratamiento SA y T3 presentó una concentración significativamente mayor a la del control (Fig. 2.1). A las $24 \mathrm{hs}$ se repiten las diferencias entre los tratamientos $(p<0,001)$. Las concentraciones son significativamente menores en el control que el tratamiento SA y son significativamente mayores en T2 y T3 que en el control. 

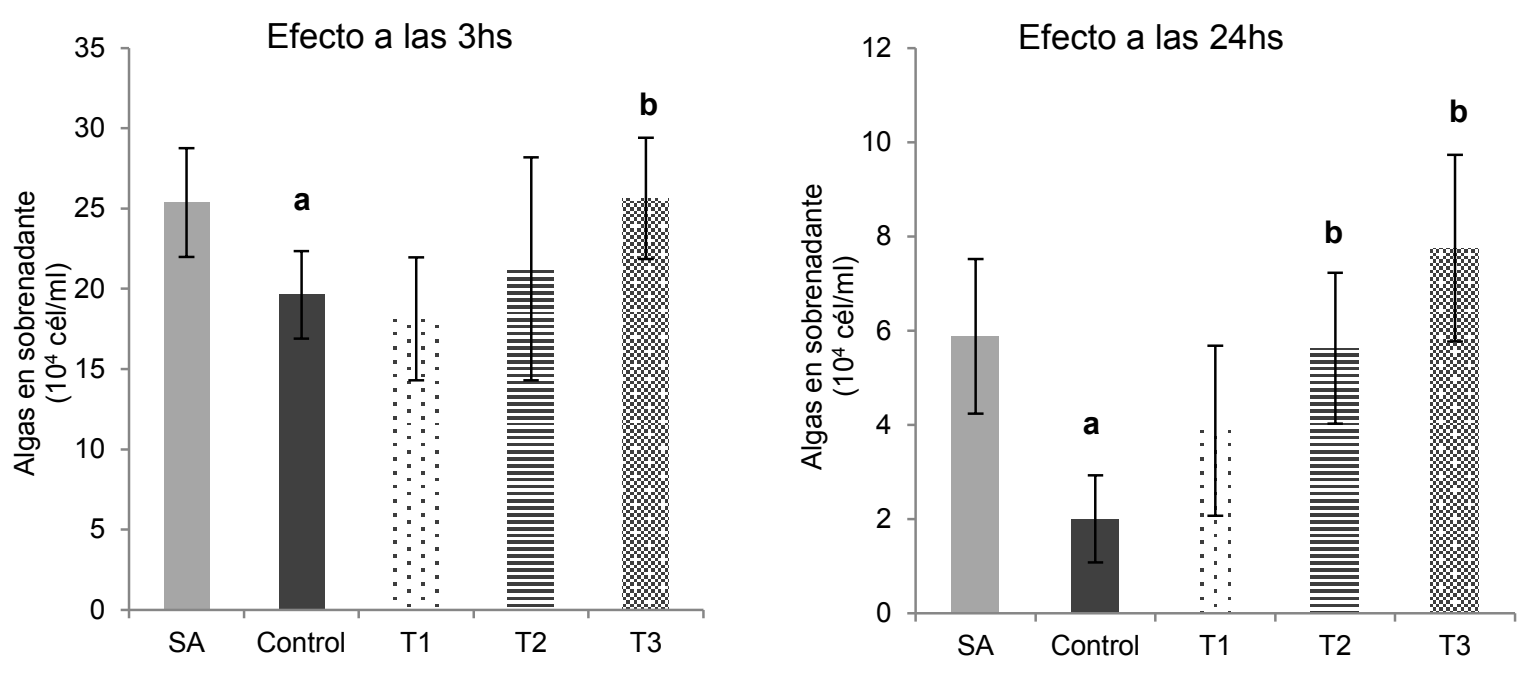

Figura 2.1. Densidad de algas no ingeridas a las 3 y 24 hs posteriores a la exposición. La letra

(a) indica diferencias significativas con SA y (b) diferencias significativas con respecto al Control (test de Dunnet, $\alpha<0,05$ ).

La disminución en la densidad de algas se debe la alimentación. A las 3 hs, el tratamiento T3 registró una densidad de algas significativamente mayor a la del control. Luego de las 24 hs posteriores a la exposición, tanto T2 como T3 fueron significativamente mayores a la del control pero no difieren del tratamiento SA, lo que sugiere la inhibición de la alimentación a las concentraciones ensayadas.

\subsection{Efectos a nivel poblacional}

\section{Diseño Experimental III: Tabla de vida}

\section{Metodología}

Organismos de $S$. vetulus de 3 días de edad fueron expuestos a pulsos de toxicidad de 90 min de cipermetrina formulado comercial Galgotrin ${ }$. Se realizaron tres tratamientos y un control, de 10 individuos cada uno, con 3 réplicas. Se utilizó la solución stock descripta en el Capítulo 1 y se utilizaron las mismas concentraciones que en el diseño experimental II. Pasado el tiempo de exposición, los organismos fueron transferidos a frascos de vidrio con $30 \mathrm{ml}$ de medio de cultivo (agua declorinada con C.vulgaris a $\left.0,5 \times 10^{6} \mathrm{cél} / \mathrm{ml}\right)$. 
Se registró diariamente el número de individuos adultos (n) y el número de individuos nacidos $(\mathrm{m})$, éstos fueron retirados luego de ser contados, dejando constante el número de individuos en cada unidad experimental $(\mathrm{N}=10)$. Se repitió la exposición cada 7 días (días 7, 14 y 21 de experimento) posteriormente al registro de los parámetros. Las condiciones del experimento fueron temperatura $22^{\circ} \mathrm{C} \pm 1^{\circ} \mathrm{C}$ y fotoperiodo natural. La renovación del medio se realizó diariamente luego del recuento para asegurar la disponibilidad de oxígeno y de alimento. El experimento finalizó al día 25.

Análisis de datos

Los datos de número de individuos $(n)$ y fecundidad $(m)$ se utilizaron para construir una tabla de vida. La tabla de vida se conoce como una tabla de vida de cohortes: una cohorte (grupo de individuos nacidos dentro del mismo intervalo de tiempo) es seguida desde el nacimiento hasta la muerte del último sobreviviente (Begon et al. 2006), si bien muchos trabajos se realizan con un punto de corte específico (ej. 21 días). La tabla de vida vincula medidas de supervivencia (Ix) y fecundidad específicas por edad $(\mathrm{mx})$ a lo largo del tiempo para estimar parámetros como la tasa intrínseca de crecimiento, tiempo de generación y la tasa reproductiva neta por generación ó R0 para un grupo de animales que crecen bajo un conjunto dado de condiciones ambientales, siendo un método válido para evaluar efectos de la exposición de los organismos a los tóxicos (Daniels y Allan 1981, Day y Kaushik 1987, Ferrando et al. 1993, Sánchez et al. 2000, Stark y Banks 2003). Se determinaron los siguientes parámetros según las fórmulas (Pianka 1982, Begon et al. 2006):

*Supervivencia (Ix): proporción de individuos que sobreviven de una edad a la otra, se obtiene una Ix para cada día de edad del individuo.

*Fecundidad (mx): número de organismos nacidos por hembra promedio, se obtiene una mx para cada día de edad de los individuos.

*Fecundidad acumulada ( $\mathrm{F}$ acum.): número total de organismos nacidos en cada tratamiento. Se calculó para el final del experimento.

*Esperanza de vida (ex): tiempo por vivir de individuo en la edad $\mathrm{x}$ 


$$
\mathrm{ex}=\frac{1}{l x} \sum_{i=x}^{\infty} l i
$$

*Tasa de reproducción bruta (RB): sumatoria de $\mathrm{mx}$

$$
\mathrm{RB}=\sum_{x=0}^{\infty} m x
$$

*Tasa de reproducción neta (R0): fecundidad respecto de la supervivencia

$$
\mathrm{R} 0=\sum_{x=0}^{\infty} l x . m x
$$

*Tiempo generacional (TG): tiempo que transcurre desde que nace un individuo hasta que nace su descendiente promedio:

$$
\mathrm{TG}=\frac{1}{\mathrm{R} 0} \sum_{x=0}^{\infty} x \cdot l x \cdot m x
$$

*Tasa de crecimiento poblacional (r) ó tasa intrínseca de crecimiento poblacional: indica de qué manera crece la población:

$$
1=\sum_{x=0}^{\infty} l x \cdot m x \cdot e^{-r x}
$$

Para evaluar diferencias entre los parámetros supervivencia y fecundidad se realizó un ANOVA MR de una vía con el software STATISTICA (Stat soft. Versión 7.0). El ANOVA MR depende de un supuesto de esfericidad, que indica que las varianzas de las diferencias son iguales. Se verificó que los datos cumplan con los supuestos de normalidad con el test de Shapiro Wilk y de esfericidad con el test de Mauchly (1940). Cuando se encontraron diferencias significativas, se utilizó el test de Bonferroni para evaluar cuáles de los factores o de los tratamientos fueron diferentes.

Para evaluar diferencias de los parámetros restantes se realizó un ANOVA de una vía con cada uno de los parámetros determinados con el software Sigma Stat 5.5. y se analizaron con un nivel de significancia de $\alpha=0,05$. Previamente se verificaron los 
supuestos de normalidad y homocedasticidad. Para evaluar cuál de los tratamientos difirió del control, se realizó un test a posteriori de Dunnet cuando el ANOVA mostró diferencias significativas.

\section{Resultados}

En la figura 2.2. se muestran las curvas de supervivencia (Ix) promedio para cada uno de los tratamientos. El ANOVA MR mostró diferencias significativas para la interacción $(F=4,66 ; p<0,001)$, para los tratamientos $(F=22,85 ; p<0,001)$ y para el tiempo $(F=78,7 ; p<0,001)$, indicando que las diferencias entre los tratamientos dependen del tiempo.

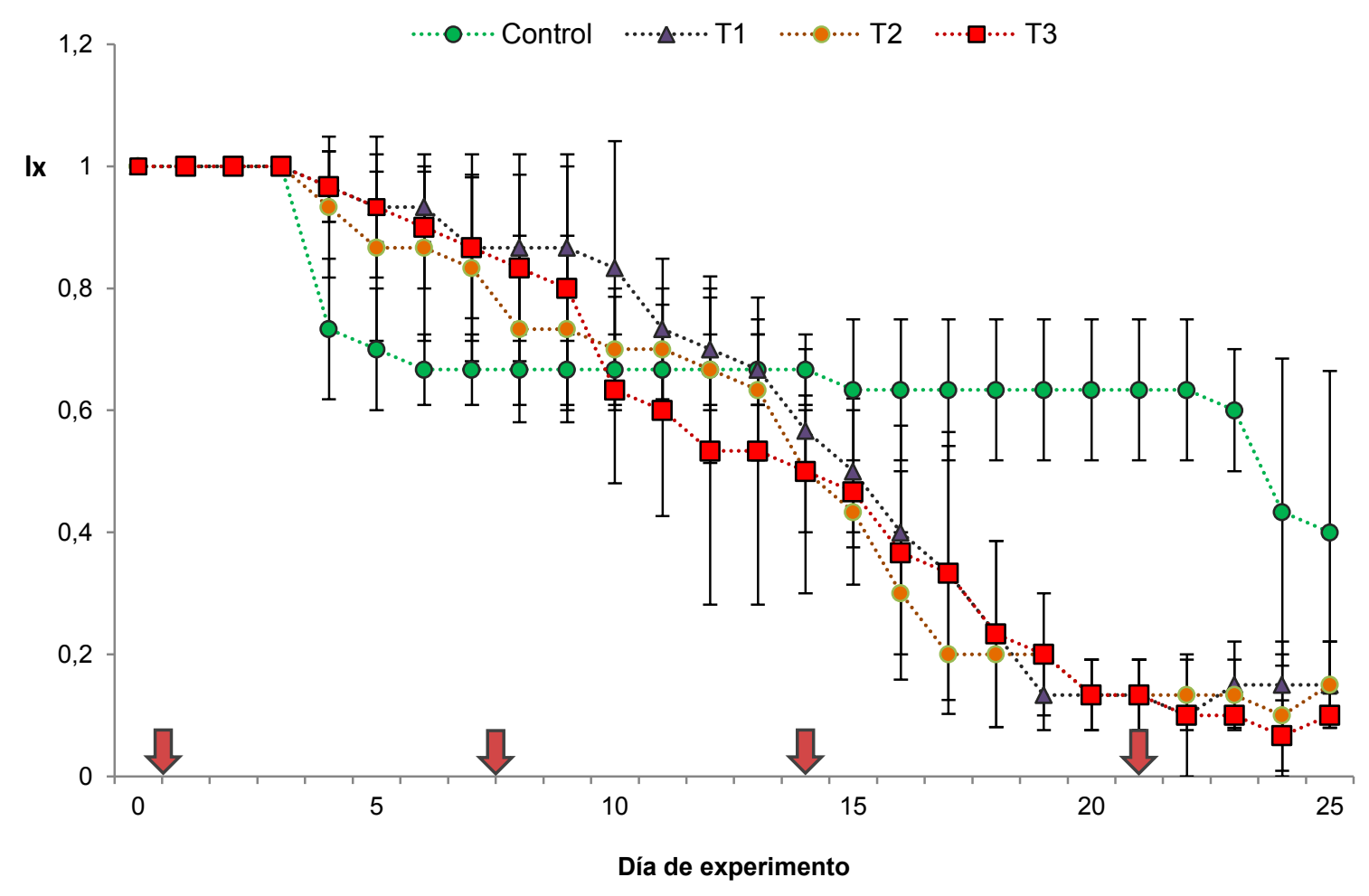

Figura 2.2. Supervivencia media y desvío estándar por tratamiento por día de experimento. Las flechas señalan las exposiciones.

En el gráfico se observó una disminución inicial de la supervivencia de los organismos del control. Sin embargo, el test a posteriori de Bonferroni indicó que estas diferencias aparentes no fueron estadísticamente significativas. La supervivencia de 
los controles se estabilizó y se mantuvo constante a partir del día 4, mientras que la supervivencia de los organismos expuestos disminuyó. Con el test de Bonferroni se determinó que, a partir del día 17, la supervivencia de los tratamientos fue significativamente diferente del control; es decir, luego de la tercera exposición disminuye la supervivencia de los organismos expuestos a cipermetrina.

La fecundidad $(m x)$ registró diferencias significativas en el tiempo ( $F=21,3$; $p<0,001)$, los tratamientos $(F=5,86 ; p<0,001)$ y la interacción $(F=2,71 ; p<0,001)$ indicando que la fecundidad varió con el tiempo en los tratamientos. El test de Bonferroni indicó que todos los tratamientos presentaron fecundidad diferente del control.

La tabla 2.2 registra los parámetros de la tabla de vida para cada tratamiento. Se observaron diferencias significativas en los parámetros de fecundidad: fecundidad acumulada, tasa de reproducción y $r$ poblacional. La fecundidad acumulada y la tasa de reproducción neta de todos los tratamientos fueron diferentes al control $(p=0,001$ y $p=0,005$ respectivamente), pero no fueron diferentes entre sí $(p>0,05)$, mientras que el valor de $r$ fue significativamente diferente al control $(p=0,032)$ sólo en el tratamiento de mayor exposición.

Tabla 2.2. Parámetros determinados en el experimento. En negrita se muestran los parámetros que mostraron diferencias significativas $(p<0,05)$ y $\left(^{*}\right)$ los que difieren del control.

\begin{tabular}{ccccccc}
\hline & $\begin{array}{c}\text { Esperanza } \\
\text { de vida }\end{array}$ & $\begin{array}{c}\text { Tiempo } \\
\text { Gener. }\end{array}$ & $\begin{array}{c}\text { Fecundidad } \\
\text { acumulada }\end{array}$ & $\begin{array}{c}\text { Tasa de } \\
\text { reproducción } \\
\text { bruta }\end{array}$ & $\begin{array}{c}\text { Tasa de } \\
\text { reproducción } \\
\text { neta (R0) }\end{array}$ & $\begin{array}{c}\mathbf{r} \\
\text { poblacional }\end{array}$ \\
\hline Control & $16,8 \pm 2,5$ & $16,4 \pm 0,4$ & $565 \pm 98$ & $105,4 \pm 10,9$ & $57,3 \pm 9,8$ & $0,25 \pm 0,02$ \\
T1 & $15,3 \pm 0,4$ & $13,9 \pm 1,8$ & $297 \pm 20\left(^{*}\right)$ & $85,3 \pm 31,3$ & $33,4 \pm 5\left(^{*}\right)$ & $0,25 \pm 0,01$ \\
T2 & $14,3 \pm 2,3$ & $14,1 \pm 2,8$ & $253 \pm 32\left(^{*}\right)$ & $82,6 \pm 34,1$ & $28,8 \pm 7,5\left(^{*}\right)$ & $0,24 \pm 0,02$ \\
T3 & $14,3 \pm 2,7$ & $14,5 \pm 2,3$ & $220 \pm 61\left(^{*}\right)$ & $86,3 \pm 35,1$ & $25,1 \pm 8,7\left(^{*}\right)$ & $0,22 \pm 0,03\left(^{*}\right)$ \\
\hline p-valor & 0,495 & 0,474 & $\mathbf{0 , 0 0 0 1}$ & 0,773 & $\mathbf{0 , 0 0 5}$ & $\mathbf{0 , 0 3 2}$ \\
\hline
\end{tabular}

En la figura 2.3 se graficó el promedio de la fecundidad acumulada de cada tratamiento. Se puede observar que el control presenta una mayor fecundidad acumulada que los tratamientos a partir de la tercer exposición (luego de los 17 días de la primer exposición). 


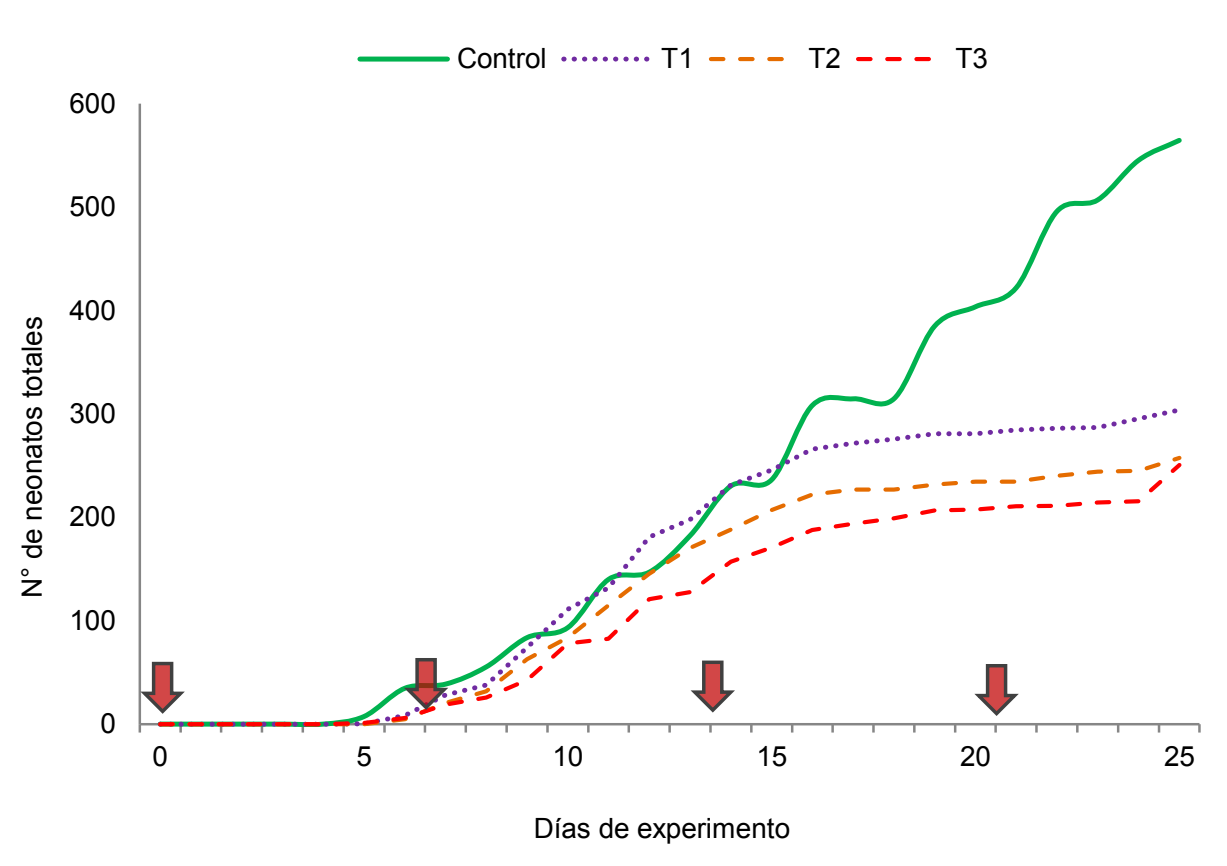

Figura 2.3. Fecundidad acumulada promedio por tratamiento por día de experimento. Las flechas señalan las exposiciones.

\section{Discusión}

En los experimentos desarrollados en el presente trabajo de tesis se determinó el efecto de reiterados pulsos de toxicidad subletales sobre $S$. vetulus tanto a nivel organísmico como a nivel poblacional. Los resultados de los experimentos a nivel organísmico (l y II) mostraron efectos en la supervivencia, en las mudas y en la alimentación.

En el diseño experimental I se determinó que organismos expuestos a pulsos breves (60 minutos) de 5 veces su concentración letal $(1 \mu \mathrm{g} / \mathrm{L})$ no registran mortalidad inmediata pero presentaron una disminución significativa de la longevidad, indicando que los organismos pueden sobrevivir a pulsos de toxicidad pero que existen efectos posteriores. Day y Kaushik (1987) observaron una disminución de la supervivencia en D. galeata mendotae Birge 1918 expuesta de manera continua a 0,01; 0,05 y 0,1 $\mu \mathrm{g} / \mathrm{L}$ de fenvalerato, mientras que Toumi et al. (2013) observaron una disminución de la supervivencia de Daphnia magna bajo exposiciones continuas de 0,16 $\mathrm{gg} / \mathrm{L}$ y 0,31 $\mu \mathrm{g} / \mathrm{L}$ de deltametrina $(30-60 \%$ de su CL50) luego de 21 días de exposición. A diferencia de estos autores, en el presente trabajo de tesis los organismos estuvieron expuestos un breve periodo de tiempo y aun así se observaron efectos en la 
supervivencia. De manera similar, en experimentos con pulsos de toxicidad, Naddy et al. (2000) determinaron una reducción del $50 \%$ de la supervivencia de $D$. magna en pulsos de $12 \mathrm{hs}$ de $0,5 \mu \mathrm{g} / \mathrm{L}$ de clorpirifos. Ashauer et al. (2007) observaron también una reducción de la supervivencia en Gammarus pulex luego de 3 pulsos de 6 hs de 1 $\mu \mathrm{g} / \mathrm{L}$ de clorpirifos cada 7 días, mientras que Cold y Forbes (2004) observaron este efecto en la misma especie en los 4 días posteriores a la exposición a un pulso de 60 $\min$ de $0,3 \mu \mathrm{g} / \mathrm{L}$ de esfenvalerato, valor doble a su CL50 (0,14 $\mu \mathrm{g} / \mathrm{L})$. La exposición a pulsos de toxicidad de plaguicidas de diferente naturaleza y modo de acción genera efectos en la supervivencia en diferentes especies, por lo tanto, las altas concentraciones aún durante breves periodos de tiempo y en repetidas ocasiones pueden generar efectos letales posteriores a la exposición en los organismos. Por otro lado, las especies presentan diferentes niveles de sensibilidad a estos compuestos, por lo que pueden responder de manera diferencial a estos pulsos, traduciéndose en efectos en la composición de los ensambles.

Existen trabajos que reportan diferentes efectos de los plaguicidas en el proceso de muda. Zou y Fingerman (1997) observaron un retraso en el proceso de muda en $D$. magna expuesta a 0,1-0,2 mg/L de endosulfán. Weis et al. (1992) recopilaron trabajos sobre los efectos de diferentes compuestos sobre el proceso de muda y hallaron que el DDT aceleró la regeneración y la muda de crustáceos, mientras que otros clorados como PCBs o diflubenzurón causaron inhibición de la regeneración. Los autores plantean que algunos plaguicidas como los organoclorados (endosulfan, DDT, aldrin, lindano, entre otros) presentan una estructura similar a las hormonas esteroideas, por lo que podrían actuar sobre los receptores de las mismas, retrasando o acelerando el proceso de muda. Por otro lado, Toumi et al. (2013) estudiaron el efecto de la exposición crónica durante 21 días del piretroide deltametrina en $D$. magna. De manera similar a los resultados del experimento I, observaron una disminución en el número acumulado de mudas y también en la longitud, por lo que concluyen que deltametrina afecta el crecimiento de $D$. magna, y que, junto al número de neonatos por adultos, el número acumulado de mudas es un indicador sensible de ecotoxicidad. En el presente trabajo de tesis, las diferencias observadas en las mudas podrían deberse a la alta mortalidad en el tratamiento de mayor concentración y no estrictamente a un efecto de cipermetrina en el proceso de muda. El estudio de los efectos de los plaguicidas sobre el proceso de muda es relevante ya que podrían verse afectados no sólo los cladóceros sino también los grupos que presenten dicho proceso como otros crustáceos o insectos acuáticos. Sería de interés profundizar el estudio de los efectos de lambdacialotrina en el proceso de muda bajo 
concentraciones o exposiciones que no generen mortalidad para lograr un seguimiento apropiado de este proceso.

El diseño experimental II mostró que la exposición a un pulso efímero de cipermetrina disminuyó la tasa de filtración en $S$. vetulus, en las primeras $3 \mathrm{hs}$ posteriores a la exposición en la mayor concentración; este efecto inhibitorio persiste hasta pasadas las $24 \mathrm{hs}$ de la exposición y se manifiesta en exposiciones a muy bajas concentraciones. Mc William y Baird (2002) estudiaron el efecto de disminución de la alimentación en Daphnia magna durante y con posterioridad a la exposición en experimentos de toxicidad de diferentes plaguicidas. Observaron que permetrina (1,5$2,7 \mu \mathrm{g} / \mathrm{L})$ y lambdacialotrina $(0,0075-0,1 \mu \mathrm{g} / \mathrm{L})$ produjeron un efecto inhibitorio tanto durante (24hs) como con posterioridad a la exposición (4hs). Christensen et al. (2005) estudiaron la tasa de filtración de $D$. magna expuesta durante $6 \mathrm{~h}, 24 \mathrm{~h}, 48 \mathrm{~h}, 3$ y 7 días a cipermetrina en concentraciones de 0,05 a $1 \mu \mathrm{g} / \mathrm{L}$. Los resultados mostraron una tendencia a reducir el contenido de clorofila en el sistema digestivo a medida que se incrementaron la concentración de cipermetrina y el tiempo de exposición. Además, observaron una recuperación posterior a la exposición aunque el tiempo requerido fue mayor a medida que aumentó el tiempo de exposición, y una disminución del peso de las daphnias a concentraciones mayores a 0,3 $\mu \mathrm{g} / \mathrm{L}$ durante 3 días. Barata et al. (2008) compararon una metodología de ensayos de inhibición de la alimentación durante $24 \mathrm{hs}$ de mezclas de 16 contaminantes y efluentes para neonatos de $D$. magna con ensayos de mortalidad estandarizados para diferentes modelos experimentales. El efecto en la alimentación fue igual o más sensible que los ensayos agudos tanto para efluentes como para las mezclas de plaguicidas, por lo que los autores proponen los ensayos de $24 \mathrm{hs}$ de inhibición de la alimentación como medida rápida de monitoreo. Los resultados obtenidos en el presente trabajo de tesis son consistentes con la bibliografía, concluyendo que la inhibición de la alimentación posterior a la exposición puede ser considerada como punto final en bioensayos de toxicidad.

En el ensayo a nivel poblacional se pudieron observar efectos que no se observaron en los experimentos a nivel organísmico, se pudieron determinar efectos en la tasa de incremento poblacional y en la reproducción ya que los organismos expuestos a cipermetrina presentaron parámetros reproductivos significativamente menores que los controles. Los reiterados pulsos de toxicidad de $90 \mathrm{~min}$ de cipermetrina disminuyeron la fecundidad, incluso en concentraciones 10 veces menores que la CL50. Tanto la fecundidad (media y acumulada) como la tasa reproductiva $R_{0}$, que relaciona la fecundidad y la supervivencia, se vieron afectadas en todas las concentraciones ensayadas. Estos resultados son similares a los informados por Kim et al. (2008), quienes expusieron neonatos y juveniles de $D$. magna a 
cipermetrina durante 21 días a concentraciones de $2 \times 10^{-7}$ a $0,2 \mu \mathrm{g} / \mathrm{L}$, siendo 0,0006 $\mu \mathrm{g} / \mathrm{L}$ su CL50 a 96hs. Observaron que exposiciones a 0,0002; 0,002 y a 0,2 $\mu \mathrm{g} / \mathrm{L}$ de cipermetrina durante 21 días mostraron un incremento del tiempo en liberar la primer camada y una reducción el tamaño de la camada y del número total de las mismas. De manera similar, Martinez-Jerónimo et al. (2013) estudiaron los efectos de la exposición continua a concentraciones de 0,$54 ; 5,4$ y $54 \mu \mathrm{g} / \mathrm{L}$ de $\alpha$-cipermetrina sobre D. schoedleri mediante los parámetros de una tabla de vida. En general, todos los parámetros determinados disminuyeron al incrementar la concentración: el tiempo promedio y la esperanza de vida disminuyeron en las mayores concentraciones, la tasa reproductiva neta fue significativamente menor en todos los tratamientos expuestos a cipermetrina $y$, al igual que el experimento presentado, la tasa de crecimiento poblacional se vio reducida sólo en la mayor concentración. En el trabajo de Toumi et al. (2013), las exposiciones de 0,16 y 0,3 $\mu \mathrm{g} / \mathrm{L}$ de deltametrina (entre un 30 y un $60 \%$ de su CL50) produjeron efectos en la supervivencia, en el crecimiento (longitud y número acumulado de mudas) y, de manera similar al presente trabajo, efectos en la tasa de crecimiento poblacional y en los parámetros reproductivos (días en liberar primer camada, número y tamaño de camadas) de $D$. magna. Sancho et al. (2017) estudiaron el efecto del insecticida etofenprox sobre $D$. magna, que presenta un mecanismo de acción similar a permetrina y cipermetrina. Los organismos fueron expuestos durante 21 días a concentraciones subletales crónicas de 0,7 a 1,85 $\mu \mathrm{g} / \mathrm{L}$ y determinaron que la mayoría de los parámetros estuvieron afectados por la exposición a las mayores concentraciones. Se registró un retraso en la maduración (días para liberar primer camada) y en la fecundidad (tamaño de la camada y número total de las mismas) en organismos expuestos a la mayor concentración. Estos efectos son similares a lo observado en el experimento III, por lo que el mecanismo de acción en la reproducción podría ser semejante con ambos tóxicos.

Los experimentos de inhibición de la alimentación 24 hs después de la exposición (II) y de efectos en la reproducción (III) resultaron complementarios ya que, como fue expresado anteriormente, la alimentación tiene un efecto directo en la fecundidad. La disminución en la eficiencia de la alimentación tiene consecuencias en el balance energético y en la reproducción como por ejemplo, efectos en el número de huevos por hembra (Gliwicz y Boavida 1996, Barata et al 2002), en el tiempo de liberación de camadas (Guisande y Gliwicz 1992) y en la frecuencia de reproducción (Urabe 1991), entre otros, por lo que este efecto inhibitorio sobre la alimentación podría alterar el proceso reproductivo y consecuentemente, la fecundidad y el crecimiento poblacional. Estas experiencias reflejan la importancia de realizar estudios a diferentes niveles de 
organización para comprender de una mejor manera los efectos de estos compuestos en los organismos.

Los resultados de los experimentos desarrollados en el presente trabajo de tesis son consistentes con numerosas referencias bibliográficas. No obstante, la mayoría de los trabajos de la bibliografía refieren a resultado de experiencias bajo exposiciones continuas a plaguicidas. En el presente capítulo se ensayaron situaciones ambientalmente realistas utilizando pulsos de toxicidad. Se determinó que $S$. vetulus sobrevive a pulsos efímeros de exposición a concentraciones comparativamente elevadas de cipermetrina y lambdacialotrina, sin registrar mortalidad inmediata, pero que esta exposición produce efectos que se manifiestan con posterioridad en los organismos y en la población: disminución de la supervivencia, menor tasa de alimentación, fecundidad acumulada, tasa de reproducción y tasa de incremento poblacional (r). Es importante señalar que los efectos en la reproducción se observaron en los organismos expuestos a muy bajas concentraciones de plaguicida, incluso en concentraciones 10 veces menores a su concentración letal.

Los resultados obtenidos permiten inferir que una población de $S$. vetulus residente en un arroyo adyacente a cultivos probablemente sobreviva inmediatamente después de un evento de escorrentía superficial pero presentará efectos en su densidad poblacional. En el capítulo siguiente se estudia la riqueza y la densidad de taxones de invertebrados en arroyos en cuencas con uso intensivo de agroquímicos y se comparan con ambientes menos impactados. 


\section{CAPÍTULO 3.}

\section{Análisis del ensamble de invertebrados en cuencas con horticultura intensiva}




\section{CAPÍTULO 3: Análisis del ensamble de invertebrados en cuencas con horticultura intensiva}

\section{Introducción}

Los ambientes acuáticos están influenciados por el uso del suelo en su cuenca (Allan 2004). Esta influencia ocurre debido a la alteración de la dinámica del agua y al transporte de contaminantes hacia ambientes adyacentes, pudiéndose determinar desde cambios en la morfología de los ríos o arroyos (Langhammer 2003, Keesstra et al. 2005), alteración de la química del agua como las concentraciones de nutrientes, efectos en la biota y cambios en los procesos ecosistémicos como la descomposición de la materia orgánica, alterando el balance energético del sistema (Young y Huryn 1999, Gücker et al. 2009). Las concentraciones de nutrientes varían según la proporción de cultivos en la cuenca (Jordan et al. 1997). Las mayores concentraciones de nitrógeno y fósforo en arroyos de Canadá se relacionan principalmente a la actividad urbana, seguido de la actividad agrícola (Thomas et al. 2018).

Del mismo modo, el uso del suelo influye en los ensambles biológicos. Roy et al. (2003) estudiaron ensambles de invertebrados en relación a la cobertura del suelo urbana, rural y con bosques prístinos. La riqueza de especies y otros índices bióticos relacionados a buena calidad del agua presentaron una relación negativa con la cobertura del suelo urbana y rural, y una relación positiva con la cobertura de bosque. Miserendino et al. (2011) estudiaron ensambles de vegetación, invertebrados, peces y aves en 18 arroyos con diferente uso del suelo en la Patagonia Andina: bosque nativo con manejo, bosque implantado, pasturas y urbanización. Los sitios urbanos presentaron menor riqueza y diversidad de invertebrados que los arroyos de bosque nativo, y los sitios de pasturas menor riqueza que los de bosque implantado. Además determinaron cambios en la concentración de nutrientes, la calidad de ribera y las condiciones del hábitat.

La agricultura se ha considerado como la principal causa de deterioro de los ambientes acuáticos (Cooper 1993, Schulz 2004). La escorrentía superficial es la principal vía de contaminación a los cuerpos de agua en cuencas agrícolas, transportando nutrientes (Hart et al. 2004) y plaguicidas (Jergentz et al. 2005) de los cultivos a los ambientes acuáticos adyacentes; estos últimos producen pulsos de toxicidad para los organismos residentes (Schulz 2001, Mugni et al. 2011). Como se mencionó en la Introducción General, la mayoría de los plaguicidas utilizados en la 
horticultura son insecticidas $(60 \%)$, en general no-selectivos, que pueden afectar también a los invertebrados acuáticos no-blanco. De todos los utilizados, clorpirifos, cipermetrina, endosulfan y su producto de degradación (endosulfán-sulfato) son los más frecuentemente detectados en estudios en la región (Hunt et al. 2016, Solis et al. 2016 y 2017).

Entre los efectos tóxicos de los plaguicidas en los organismos se encuentran la disminución de la supervivencia o de la reproducción, como fue desarrollado en el capítulo anterior. Otros efectos no relacionados directamente a la toxicidad de agroquímicos, tales como la degradación del hábitat y la disminución de la calidad del agua, pueden afectar a las especies poco tolerantes, resaltando la importancia de realizar investigaciones en los ensambles biológicos residentes en los arroyos.

Varios estudios de campo relacionan la exposición a plaguicidas con cambios en la estructura de la comunidad de invertebrados acuáticos, en general la desaparición o la disminución de la densidad de taxones considerados sensibles. Schulz y Liess (1999) registraron pulsos de contaminación en arroyos debido a la escorrentía superficial de los cultivos adyacentes y además, observaron la desaparición de 8 de 11 especies de invertebrados luego de un pulso de $6 \mu \mathrm{g} / \mathrm{L}$ de paratión y la mortalidad de Gammarus pulex (anfípodo) y Limnephilus lunatus Curtis, 1834 (tricóptero) causado por pulsos de contaminación de $6,2 \mu \mathrm{g} / \mathrm{L}$ de fenvalerato. Berenzen et al. (2005) estudiaron el efecto de variables ambientales y plaguicidas en la comunidad de invertebrados acuáticos en arroyos de Alemania. De las variables estudiadas, sólo los plaguicidas (definidos en base a unidades tóxicas) explicaron significativamente la comunidad de invertebrados acuáticos; Trichoptera, Ephemeroptera y Plecoptera se correlacionaron negativamente con la concentración de plaguicidas. Sumado a esto, Schäfer et al. (2007) determinó que los plaguicidas afectaron tanto la abundancia de especies como al proceso de descomposición de la hojarasca, debido a la disminución de la abundancia de taxones ramoneadores sensibles, por lo que la exposición a plaguicidas afecta no solo a la composición del ensamble sino también a las funciones del ecosistema.

La sensibilidad diferencial de los invertebrados a los plaguicidas puede ser utilizada como una herramienta de biomonitoreo para evaluar el estado de los cuerpos de agua. Liess y Van der Ohe (2005) establecieron una clasificación de las especies de invertebrados en dos categorías: especies en riesgo o especies sensibles (SPEAR: SPecies At Risk) y especies no-en riesgo o tolerantes (SPEnotAR: SPecies Not At Risk). Este criterio, elaborado a nivel específico, presenta una aplicabilidad válida a 
nivel de familia (Beketov et al. 2009) y ha sido utilizado en otras regiones del mundo (Schäfer et al. 2012) incluso en nuestro país (Hunt et al. 2017).

En trabajos previos en la zona se determinaron concentraciones de plaguicidas y se determinó que el uso del suelo agrícola afecta a los ensambles de invertebrados acuáticos en arroyos pampeanos (Solis et al. 2017 y 2018) resultando en la desaparición o disminución de la abundancia de grupos sensibles como odonatos y efemerópteros (Jergentz et al. 2004) o anfípodos (Solis et al. 2016). Por el contrario, el efecto del uso del suelo hortícola sobre el ensamble de invertebrados en arroyos no ha sido estudiado. Como antecedentes de estudios llevados a cabo en el Cinturón Hortícola Platense pueden citarse a Mac Loughlin et al. (2017) quienes determinaron la presencia de plaguicidas y toxicidad de los sedimentos para Hyalella curvispina en el arroyo Carnaval, mientras que Rimoldi et al. (2018) estudiaron la cuenca del arroyo del Gato donde determinaron la presencia de plaguicidas en las nacientes del mismo.

En cuencas con horticultura intensiva, los agroquímicos utilizados en los cultivos podrían alcanzar los cuerpos de agua adyacentes, generando efectos en la concentración de nutrientes y en los ensambles de invertebrados. El estudio de campo a nivel comunitario y ecológico se podrá relacionar con los estudios previos a nivel organísmico y poblacional del presente trabajo de tesis y, de este modo, lograr un enfoque transversal del efecto de la horticultura en los arroyos.

\section{1. Área de Estudio}

Se estudiaron 8 arroyos cercanos a La Plata y Magdalena, Buenos Aires (fig. 3.1), pertenecientes a la vertiente del Río de la Plata. Estos arroyos se ubican subparalelos entre sí, son de bajo orden y corren en dirección SO-NE. El relieve comprende dos zonas bien diferenciadas (Hurtado 2006): la Llanura Alta, desde los 5 a $30 \mathrm{~m}$ s.n.m., terrenos con muy suaves ondulaciones conformados por sedimentos loéssicos eólicos de origen continental; y la Llanura Costera, desde los 5 m.s.n.m hasta el nivel del mar, conformada por limos y arcillas estuáricos correspondientes a depósitos marinos del Holoceno (cordón de conchilla). Los arroyos en general desembocan en la llanura costera donde se insumen o se distribuyen superficialmente sobre esta planicie, formando bañados o, en la zona de la ciudad de La Plata, son canalizados para facilitar su escurrimiento hacia el Río de La Plata. La pendiente es escasa $(<1 \%)$, los arroyos son poco profundos con baja velocidad de la corriente (flujo laminar) y carecen de un bosque de ribera. En las márgenes se desarrollan macrófitas y abundante vegetación riparia (Giorgi et al. 2005). El clima es templado húmedo, la 
temperatura media anual es de $16^{\circ} \mathrm{C}$, de $10^{\circ} \mathrm{C}$ en Julio a $23^{\circ} \mathrm{C}$ en Enero. La precipitación media anual es alrededor de $1000 \mathrm{~mm}$, la más alta en marzo $(111 \mathrm{~mm})$ y la más baja en Junio (63 mm) (Hurtado et al. 2006).

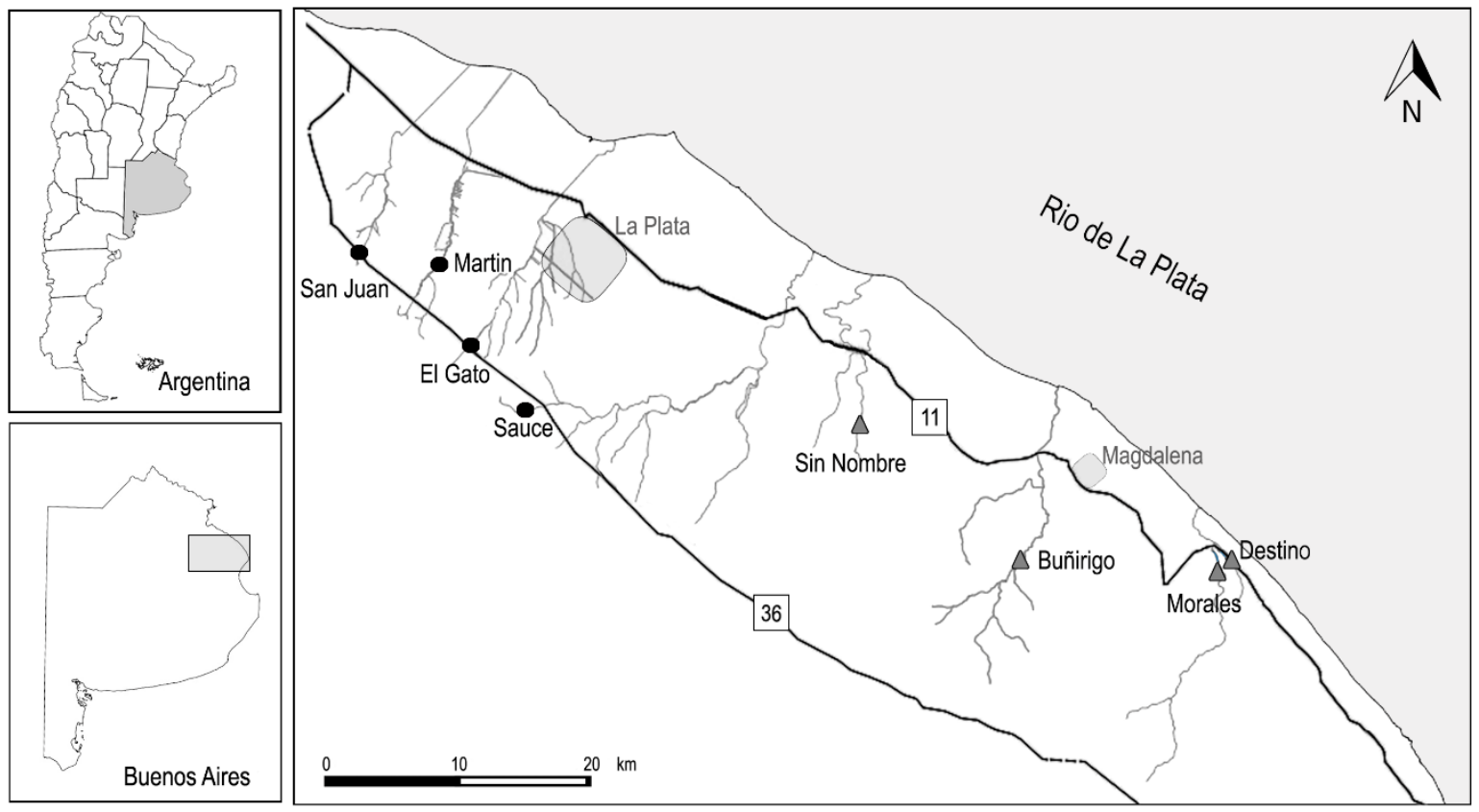

Figura 3.1. Área de estudio y sitios de muestreo.

Se seleccionaron cuatro arroyos en cuyas cuencas se desarrolla horticultura intensiva: San Juan, Martín, Gato y Sauce, localizados dentro del Cinturón Hortícola de los alrededores de La Plata. Otros cuatro arroyos se consideraron como sitios de referencia: dos drenan cuencas con pastizales naturales y ganadería extensiva (Buñirigo y Sin Nombre) y otros dos ubicados en la Reserva de Biósfera de la UNESCO Parque Costero del Sur (Destino y Morales) (Athor 2009), con actividad ganadera, turística, recreativa y educativa. Solis et al. (2018) determinaron que la composición del ensamble de la reserva y de arroyos con ganadería extensiva no fue significativamente diferente, por lo que este grupo de arroyos se analizó en conjunto.

\section{Arroyos en cuencas hortícolas}

\section{Arroyo San Juan}

El sitio de muestreo se localiza en la localidad de Arturo Seguí, Partido de La Plata, cercano a la Ruta Provincial N 36 (R. 36) (3454'39"S, 5809'07"O). Se observan emprendimientos hortiflorícolas en las cercanías al sitio de muestreo (fig. 3.2.a). El cauce es poco profundo y con fondo lodoso. La vegetación del cauce está 
conformada por carpetas de vegetación flotante de Lemna gibba L. y Azolla filiculoides Lam., acompañado de Ludwigia peploides (Kunth) P.H. Raven y Sagittaria montevidensis Cham. \& Schltdl. (fig. 3.2.b). En las márgenes se observa la presencia de arbustos y árboles como Parkinsonia aculeata L.

\section{Arroyo Martín}

Sitio de muestreo localizado en la ciudad de City Bell, Partido de La Plata,

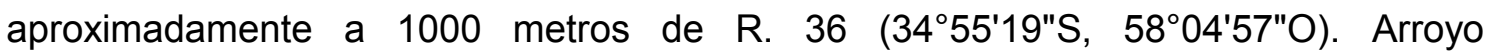
generalmente angosto y de poco caudal (fig. 3.2.c) el fondo es lodoso con gravas pequeñas. Se observó la presencia de ganado vacuno de manera esporádica. Presenta abundante vegetación riparia y poca vegetación acuática, formando parches aislados de vegetación emergente como Gymnocoronis spilantoides (D. Don ex Hook. \& Arn.) DC. y Polygonum punctatum Elliott (fig. 3.2.d). No presenta árboles en las márgenes.

\section{Arroyo del Gato}

Sitio de muestreo localizado en la localidad de Lisandro Olmos, Partido de La Plata, cercano a R. $36\left(34^{\circ} 59^{\prime} 01^{\prime \prime} S 58^{\circ} 03^{\prime} 14 " O\right)$. El cauce se encuentra rectificado (fig. $3.2 \mathrm{e})$, con no más de $4 \mathrm{~m}$ de ancho. La vegetación dominante es arraigadasumergida, conformada por $L$. peploides y Egeria densa Planch., acompañados por $P$. punctatum, Hydrocotyle ranunculoides L. f. y algas filamentosas (fig. $3.2 \mathrm{f}$ ). El fondo es lodoso con abundante materia orgánica (vegetación en descomposición). Se observan árboles aislados y vegetación espontánea en las márgenes.

\section{Arroyo El Sauce}

Sitio ubicado al sur de la R. 36 en la localidad de Lisandro Olmos, Partido de La Plata $\left(35^{\circ} 01^{\prime} 412^{\prime \prime S} 58^{\circ} 00^{\prime} 25^{\prime \prime} \mathrm{O}\right)$. Es uno de los arroyos que dan origen al $\mathrm{A}^{\circ} \mathrm{El}$ Pescado, que desemboca en la planicie costera. La naciente es cercana al punto de muestreo. Atraviesa una zona de horticultura intensiva, los invernaderos están dispuestos muy próximos al curso de agua $(3.2 \mathrm{~g})$. No se registró la presencia ganado en ningún muestreo. El curso es permanente, en general con poca velocidad de corriente y poca vegetación (fig. 3.2. h), dominada por especies emergentes como $G$. spilantoides, P. punctatum. y L. peploides, generando parches arraigados. Aparecen árboles exóticos en la ribera (Gleditsia triacanthos L.) generando sombra en el cauce. Las márgenes presentan gran desarrollo de vegetación espontánea (ej.: Senecio bonariensis Hook. \& Arn.). 

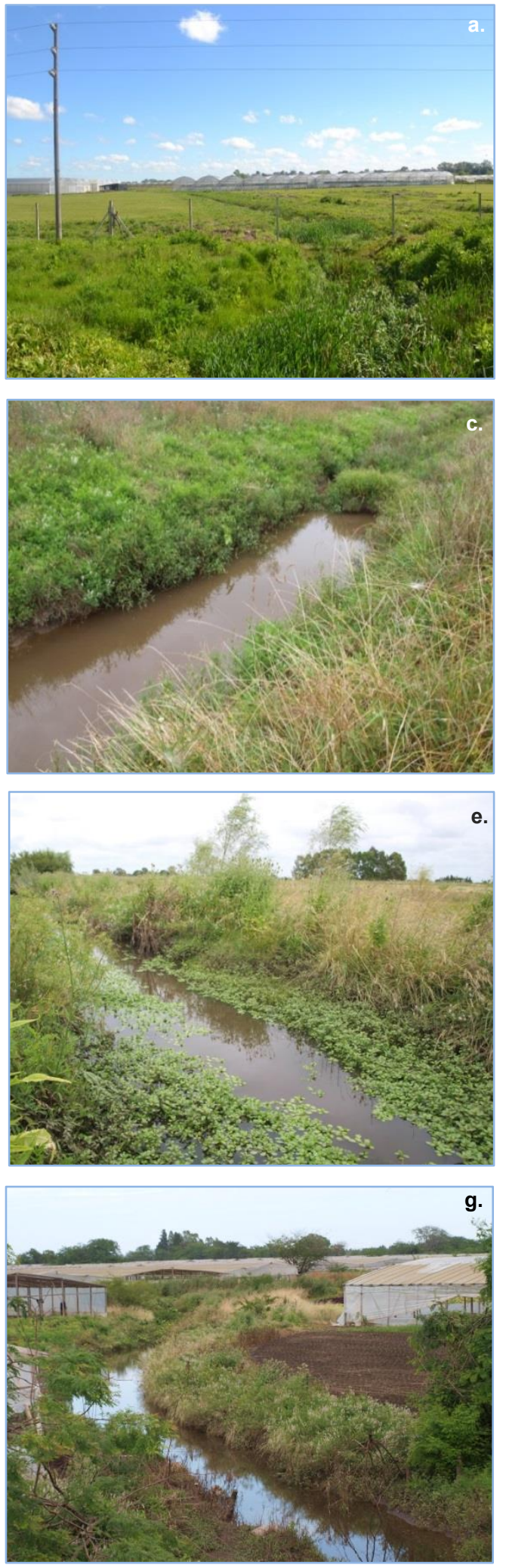
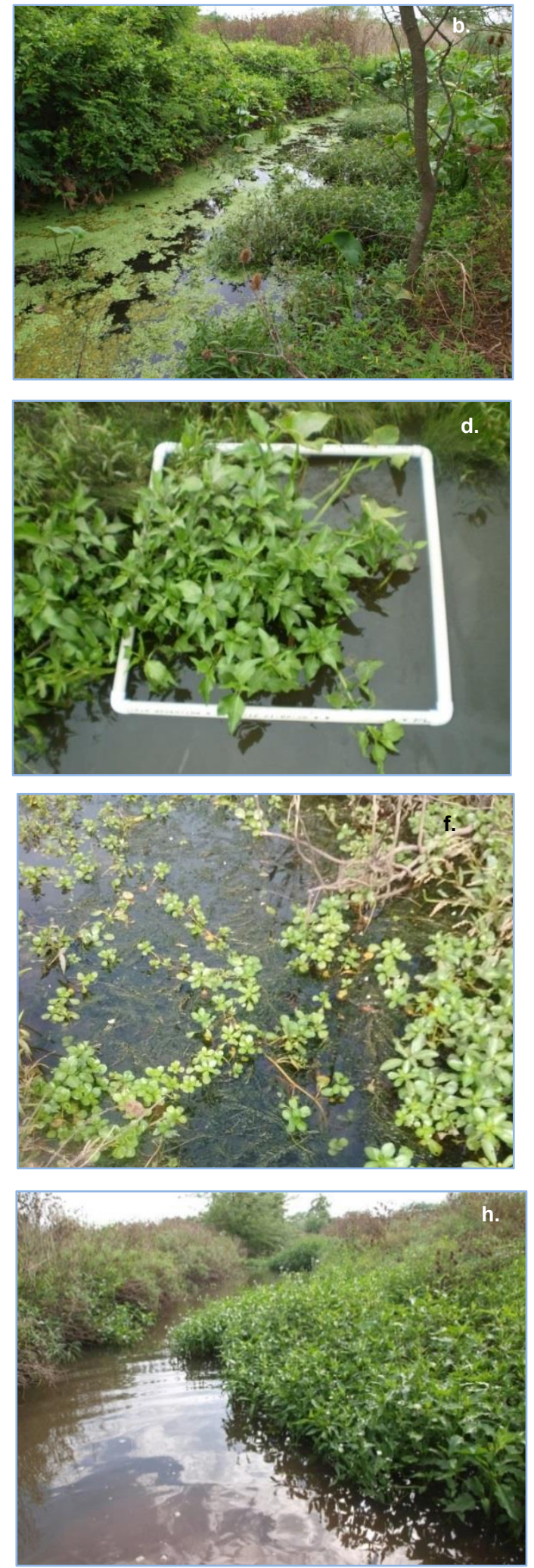

Figura 3.2. Arroyos Hortícolas. $A^{\circ}$ San Juan: a.) invernaderos en el cauce y b.) cauce y vegetación. $A^{\circ}$ Martín: c.) cauce y d.) vegetación. $A^{\circ}$ del Gato: e.) cauce y f.) vegetación. $A^{\circ}$ El Sauce: g.) cauce e invernaderos y h.) vegetación. 


\section{Arroyos de referencia: sin horticultura en sus cuencas}

\section{Arroyo Sin Nombre}

Sitio de muestreo localizado en la localidad de Gral. Mansilla, Partido de

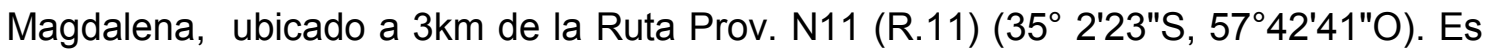
afluente del $\mathrm{A}^{\circ}$ Zapata. Atraviesa una zona de ganadería extensiva con presencia de ganado en el cauce, pudiendo ingresar libremente al mismo. Márgenes de césped ribereño con algunos ejemplares de Celtis tala Gillies ex Planch., pero sin sombra en el cauce. Vegetación acuática compleja y diversa, dominada por Myriophyllum aquaticum (Vell.) Verdc., L. peploides y G. spilantoides, acompañado por Potamogeton gayi A. Benn e Hydrocleys nymphoides (Willd.) Buchenau (fig. 3.3 b). En ocasiones presencia de algas filamentosas del género Spyrogyra.

\section{Arroyo Buñirigo}

Sitio de muestreo localizado cerca de la localidad de Magdalena, ubicado a la

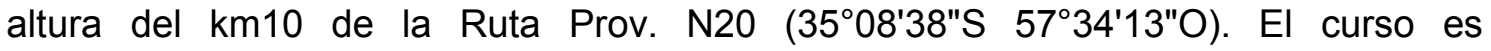
permanente, presenta una leve corriente y puede alcanzar profundidades de casi $1 \mathrm{~m}$. Es una zona de ganadería extensiva, el ganado tiene acceso limitado al arroyo (alambrado discontinuo). El tipo de vegetación dominante fue arraigado-sumergida, caracterizado por Schoenoplectus californicus (C.A. Mey.) Soják, L. peploides y Ceratophyllum demersum L., acompañadas por G. spilantoides, $H$. nymphoides y algas filamentosas (fig. $3.3 \mathrm{~d}$ ).

\section{Arroyo Morales}

Sitio de muestreo localizado dentro de la Reserva Parque Costero del Sur, Partido de Magdalena, cercano a la Ruta Prov. N 11 (3508'24"S 57²4'04"O). Dentro de la reserva se une con el $A^{\circ}$ Destino y desemboca al Rio de la Plata. En la Reserva hay un manejo de ganado extensivo, con acceso al arroyo limitado. El tipo de vegetación dominante fue flotante del tipo carpeta vegetal, conformada por varias especies y grupos diferentes (fig. $3.3 \mathrm{f}$ ). Las dominantes fueron $A$. filiculoides y $L$. gibba, fueron frecuentes G. spilantoides, L. peploides y Limnobium laevigatum (Humb. \& Bonpl. ex Willd.) Heine, y estuvieron presentes Wolffia sp., Ricciocarpus sp., Utricularia sp. y algas filamentosas. La ribera es de pastizal nativo, sin sombreado. 

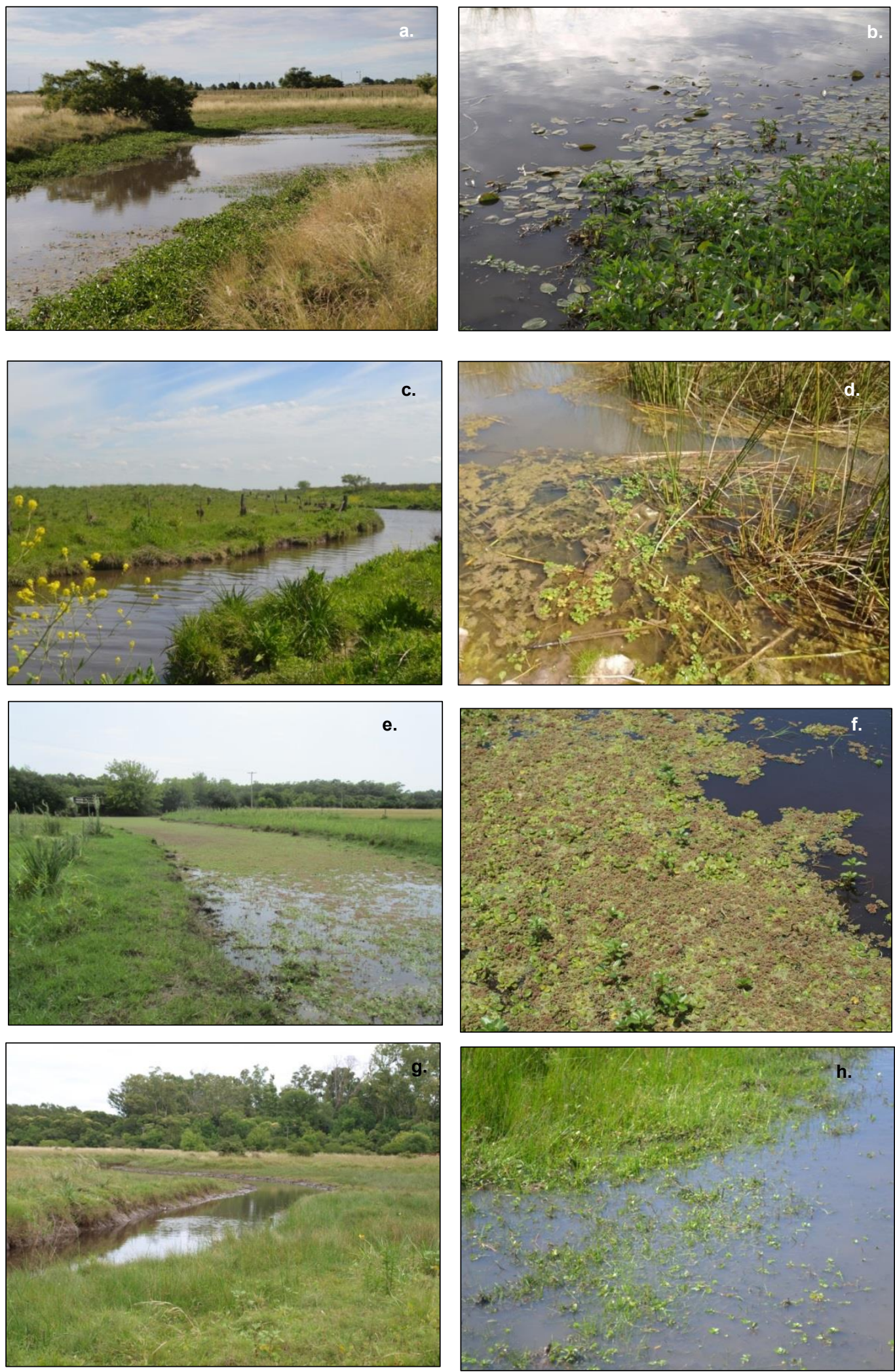

Figura 3.3. Arroyos de Referencia. $A^{\circ} \operatorname{Sin}$ Nombre: a.) cauce y b.) vegetación. $A^{\circ}$ Buñirigo: c.) cauce y d.) vegetación. $A^{\circ}$ Morales: e.) cauce y f.) vegetación. $A^{\circ}$ Destino: g.) cauce y h.) vegetación. 


\section{Arroyo Destino}

Sitio de muestreo localizado dentro de la Reserva Parque Costero del Sur, desemboca al Río de la Plata $\left(35^{\circ} 08^{\prime} 16^{\prime \prime} \mathrm{S}, 57^{\circ} 23^{\prime} 34.1^{\prime \prime O}\right)$. El tipo de vegetación dominante fue arraigado-flotante, caracterizado por L. peploides, acompañada por $M$. aquaticum, $P$. gayi y ocasionalmente algas filamentosas (fig. $3.3 \mathrm{~h}$ ). En la ribera se desarrollan pequeños montes de $C$. tala pero sin generar sombreado sobre el arroyo. Ocasionalmente presenta corrientes rápidas pero en general presenta un flujo laminar al igual que los demás arroyos.

\subsection{Análisis del ensamble de invertebrados asociados a vegetación}

\section{Metodología}

\section{Análisis espacial}

Se realizó un análisis espacial con el Software Libre Quantum GIS 2.18 (QGIS 2015) para cuantificar el área de las parcelas hortícolas dentro de las cuencas de los arroyos. Se delimitaron las cuencas de los arroyos San Juan, Martín, Gato y El Sauce en base a los datos disponibles del banco de datos del Instituto Geográfico Nacional (IGN 2015): provincia de Buenos Aires, partidos y cursos de agua de la provincia de Buenos Aires; junto a los mapas de hidrología superficial del partido de La Plata y usos del suelo, cuencas de drenaje y geomorfología elaborados por Hurtado (2006). Se utilizó también un Modelo de Elevación Digital (Digital Elevation Model, DEM) de la zona de estudio descargado del USGS (2015). El sistema de referencia utilizado para la visualización en QGIS de la información detallada anteriormente fue WGS 84 (EPGS: 4326).

Para la digitalización de las cuencas de drenaje, se georreferenciaron los mapas de cuencas de drenaje y geomorfología de Hurtado (2006) a partir del archivo vectorial del partido de La Plata. Se generó una nueva capa vectorial y se digitalizaron cada una de las cuencas, corroborando esta delimitación con el DEM para la zona y con el mapa de geomorfología. Para realizar la digitalización de parcelas con horticultura intensiva se trabajó con el complemento OpenLayer plugin y se utilizó la capa de base Google Hybrid Maps, que combina información tanto de calles como de imagen satelital. Se digitalizaron sólo las parcelas con horticultura intensiva localizadas dentro de las cuencas de los arroyos. Se utilizó la capa vectorial generada de las cuencas. Se trabajó en una escala de 1:6000. 
Para el cálculo de áreas, tanto la capa vectorial de las cuencas como la de las parcelas se reproyectaron en el sistema de proyección de coordenadas planas Campo Inchauspe - Argentina 6. Se determinaron las áreas en hectáreas para cada ítem de cada uno de los archivos vectoriales, se obtuvo la superficie de cada una de las cuencas y la superficie hortícola. Con esta información se calculó el porcentaje de cada cuenca ocupado por horticultura de la siguiente manera:

(\%) ocupado por horticultura $=\frac{\text { parcelas con horticultura (ha) } \times 100}{\text { área de la cuenca (ha) }}$

Con estos datos se realizó un mapa con los cursos de agua, las delimitaciones de las cuencas de los arroyos y las parcelas con horticultura para cada una de ellas en el sistema de referencia WGS84 (EPGS: 4326).

\section{Diseño de muestreo}

El muestreo se realizó durante la época de mayor aplicación de agroquímicos, desde la primavera hasta el otoño entre los años 2014 y 2017, en dos periodos. El primer periodo abarcó muestreos desde Octubre del año 2014 a Diciembre del año 2015, y el segundo periodo desde Noviembre de 2016 a Marzo de 2017.

\section{Variables Ambientales}

Se midieron in situ el oxígeno disuelto y la temperatura con oxímetro YSI 51B; el pH con Hanna Checker y la conductividad con conductímetro Hanna Instruments 8733. Se tomaron muestras de agua por triplicado, fueron colocadas en frío y transportadas al laboratorio, donde fueron filtradas a través de filtros Whatman GF/C. Los nutrientes se determinaron en el filtrado por colorimetría. El Fósforo Reactivo Soluble (SRP) se determinó por colorimetría de la reacción con molibdato-ascórbico, nitritos $\left(\mathrm{N}^{\left.-\mathrm{NO}_{2}{ }^{-}\right)}\right.$ por diazotación, nitratos $\left(\mathrm{N}-\mathrm{NO}_{3}{ }^{-}\right)$por reducción con hidrazina y posterior diazotación y amonio $\left(\mathrm{N}^{-} \mathrm{NH}_{4}^{+}\right)$por el método del indofenol azul (APHA, 2012). Los sólidos en suspensión (SS) se determinaron por la diferencia de peso en los filtros antes y luego de la filtración. La clorofila se extrajo con acetona luego de $24 \mathrm{hs}$ de refrigeración siguiendo a Lorenzen (1967).

Se tomaron muestras de sedimento, que fueron almacenadas en frascos de vidrio rotulados y puestas en frío para su transporte al laboratorio, donde se refrigeraron hasta el análisis de plaguicidas. El porcentaje de carbono orgánico del sedimento fue determinado por diferencia de peso por el método de incineración en 
mufla; una porción de sedimento se seca previamente a $105^{\circ} \mathrm{C}$ y luego se incinera en mufla a $550^{\circ} \mathrm{C}$ durante $2 \mathrm{~h}$ (Oliver et al., 2001). El porcentaje de cobertura de la vegetación se estimó de manera visual (Dethier et al., 1993) en el mismo área de un metro cuadrado (dividida en cuatro cuadrantes iguales) donde se muestrearon los invertebrados. Se elilgieron tres parches de vegetación similares en composición y dimensión (porcentaje de cobertura dentro del cuadrante), y dentro del mismo tramo del arroyo.

\section{Plaguicidas}

Las muestras de sedimento se extrajeron con una mezcla de acetona y cloruro de metileno, de acuerdo a la metodología descripta por You et al (2004). El clean-up se realizó con un procedimiento de extracción en fase sólida, utilizando relleno de Florisil@. El extracto se evaporó con corriente de nitrógeno y se resuspendió en $1 \mathrm{ml}$ de hexano para posterior determinación por cromatografía gaseosa.

Para el primer periodo se realizó un screening de 23 plaguicidas: organoclorados $\alpha, \omega, \beta$ y $\delta-B H C$, heptacloro y heptacloro-epóxido, aldrin, dieldrin, endrin y endrin aldehído, metoxicloro, endosulfanes ( $\alpha, \beta-$ y sulfato) y DDTs (pp'-DDD, pp'DDE, pp'DDT); el organofosforado clorpirifos y los piretroides $\lambda$-cialotrina, permetrinas, cipermetrinas (mezcla), fenvalerato y deltametrina. Los extractos en hexano se inyectaron en un cromatógrafo GC/NPD para la determinación de residuos de organofosforados (OF) y en un GC/ $\mu E C D$ para organoclorados $(O C)$ y piretroides. La cuantificación se realizó por el método de estándar externo. Los residuos de organofosforados se confirmaron por GC/MSD, y los organoclorados y piretroides se confirmaron por inyección en una columna de diferente polaridad.

Para la cuantificación de OF Se utilizó un cromatógrafo gaseoso Agilent 6890 equipado con un inyector automáticor Agilent 7683 , una columna capilar, H5 (30 m x $0,25 \mathrm{~mm}$ d.i., $0,25 \mu \mathrm{m}$ ), y un detector de nitrógeno-fósforo (GC/NPD) (temperatura del detector $300^{\circ} \mathrm{C}$, temperatura del horno programada de $70^{\circ} \mathrm{C}$ a $240^{\circ} \mathrm{C}$; temperatura de inyección $250^{\circ}$; volumen de inyección $1 \mu$ l, carrier: $\mathrm{N}_{2}$ ).

Para la cuantificación de OC y piretroides, se utilizó un cromatógrafo gaseoso Agilent $6890 \mathrm{~N}$ equipado con un inyector automático Agilent 7683B, una columna HP 5MS/DB17 (30 m x 0,25 mm d.i.; $0.25 \mu \mathrm{m}$ ) y un detector $\mu \mathrm{ECD}$, (temperatura del detector: $300^{\circ} \mathrm{C}$, programa de temperatura del horno de: $70^{\circ} \mathrm{C}$ a $240^{\circ} \mathrm{C}$; temperatura de inyector $250^{\circ} \mathrm{C}$; volumen de inyección: $1 \mu \mathrm{l}$; carrier: $\mathrm{N}_{2}$ ).

La confirmación se llevó a cabo con un detector selectivo de masas (MSD) (sistema de inyección de temperatura de vaporización programada (PTV) volumen de 
inyección $5 \mu \mathrm{l}$; carrier: $\mathrm{He}$ ). Con respecto a la linearidad de las curvas de calibración, el coeficiente $R^{2}$ fue siempre $\geq 0,99$. Se utilizaron estándares de referencia Accustandard $\circledR$ (pureza > 99\%). Los límites de cuantificación fueron $0,7 \mathrm{ng} / \mathrm{g}$ peso seco (p.s.) para organoclorados; $0,9 \mathrm{ng} / \mathrm{g}$ peso (p.s.) clorpirifos; $4,5 \mathrm{ng} / \mathrm{g}$ (p.s.), 9,1 ng/g (p.s.) para cipermetrina y 1,8-3,6 ng/g (p.s.) para otros piretroides.

Para el segundo periodo se realizó un screening de 14 pesticidas: un organofosforado (clorpirifos) y 13 organoclorados: hexaclorociclohexanos ( $\alpha-\mathrm{HCH}$ y$\mathrm{HCH}$ ), diclorodifenil-tricloroetano (DDT) sus homólogos y metabolitos (DDD y DDE), y ciclodienos (dieldrín, $\alpha$ - clordano, $y$-clordano, $\alpha$-endosulfán, $\beta$-endosulfán, endosulfán sulfato, heptacloro y heptacloro epóxido). Los extractos en hexano previamente purificados por cromatografía de adsorción en sílica gel, se inyectaron en un cromatógrafo GC-ECD para la determinación de plaguicidas. La cuantificación se realizó en base a la metodología de Miglioranza et al. (2003).

La cromatografía de adsorción con sílica gel se basa en la polaridad de las moléculas: las moléculas más polares se adsorben a la sílica quedando retenidas, a medida que las más no polares van eluyendo. Cambiando la polaridad de la fase móvil (solvente) se pueden remover las moléculas adsorbidas. Se utilizó una columna de 7 $\mathrm{cm}$ de alto y $1 \mathrm{~cm}$ de diámetro a la cual se rellenó con $1 \mathrm{~g}$ de sílica gel, previamente activada a $200^{\circ} \mathrm{C}$ por 24 hs. La columna se acondicionó con $5 \mathrm{ml}$ de hexano y posteriormente se sembró la muestra $(1 \mathrm{ml})$. Se eluyó en primer lugar con $10 \mathrm{ml}$ de hexano y luego con $20 \mathrm{ml}$ de hexano: diclorometano (1: 1, v / v). El volumen aprox. $30 \mathrm{ml}$ se recolectó en una probeta y se transfirió a un balón para su posterior concentración a $40^{\circ} \mathrm{C}$ con bomba de vacío y corriente de Nitrógeno AP, hasta un volumen final de $1 \mathrm{ml}$. Por último este $\mathrm{ml}$ se transfirió a un vial para su posterior análisis por cromatografía gaseosa con detector de captura electrónica (GC-ECD). Previo a la inyección en el cromatógrafo las muestras fueron tratadas con Cu activado para extraer los sulfuros, los cuales afectan al detector. La metodología de purificación fue tomada de Metcalfe y Metcalfe (1997) con modificaciones de Miglioranza et al. (2003).

Se utilizó un cromatógrafo gaseoso con detector de captura electrónica Shimadzu-17A equipado con detector de ${ }^{63} \mathrm{Ni}\left(290^{\circ} \mathrm{C}\right)$ y columna capilar DB-5 de Supelco (30 m x 0,25 mm d.i.; $0.25 \mu \mathrm{m}$ ) operada en modo de inyección "splitless". A fin de optimizar la separación e identificación de los compuestos en estudio, se estableció el siguiente programa de temperaturas: inicio del proceso a $100^{\circ} \mathrm{C}$ con calentamiento durante $1 \mathrm{~min}$. seguido por un incremento de la temperatura de $5^{\circ} \mathrm{C} / \mathrm{min}$ hasta $150^{\circ} \mathrm{C}$ durante $1 \mathrm{~min}$. Luego el ascenso de temperatura fue a $1,5^{\circ} \mathrm{C} / \mathrm{min}$ hasta $240^{\circ} \mathrm{C}$ para 
finalmente alcanzar $290^{\circ} \mathrm{C}\left(10^{\circ} \mathrm{C} / \mathrm{min}\right)$ donde permaneció por $10 \mathrm{~min}$. El transporte de los compuestos dentro de la columna capilar se realiza por medio de Helio AP, con velocidad de $1,5 \mathrm{ml} / \mathrm{min}$.

Los diferentes compuestos se identificaron por medio de estándares externos a través de sus tiempos de retención y utilizando el \#PCB 103 como estándar interno (Miglioranza et al., 2003). Para estimar la calidad de los datos analíticos obtenidos, se realizaron análisis de blancos y muestras fortificadas. Se usaron estándares de plaguicidas de Ultra Scientific $®$ y PCB \#103 de Accustandard Absolute Standards $®$, INC, CT, USA. El límite de detección estuvo entre 0,003 y 0,004 ng/g (p.s.) para HCHs y entre $0,007-0,029 \mathrm{ng} / \mathrm{g}$ (p.s.) para el resto de compuestos.

\section{Muestreo de invertebrados}

El muestreo se llevó a cabo con una red tipo D-net de $500 \mu \mathrm{m}$ de apertura de malla sobre la vegetación acuática, en un cuadrante flotante de PVC de $1 \mathrm{~m}^{2}$. Se tomaron tres réplicas en cada sitio de muestreo luego de registrar la cobertura y composición de la vegetación de cada una. Los organismos se colectaron y se fijaron in situ con alcohol $96 \%$. En el laboratorio, los organismos se pasaron por un tamiz de $500 \mu \mathrm{m}$, se separaron, se contaron y se identificaron hasta el menor nivel taxonómico posible, bajo microscopio estereoscópico Leica ${ }^{\circledR}$ EZ4. Se siguieron claves taxonómicas generales (Merrit et al., 2008), regionales (Domínguez y Fernández, 2009) y particulares para algunos grupos como colémbolos (Bernava Laborde y Palacios Vargas 2008), coleópteros (Oliva et al. 2002) y moluscos (César et al. 2000).

\section{Análisis de datos}

Se calcularon para cada sitio en cada fecha de muestreo, la densidad media de cada taxón (individuos $/ \mathrm{m}^{2}$ ) y las métricas riqueza taxonómica (S), densidad total promedio de individuos y diversidad de Shannon $\left(H^{\prime}\right)$ según la fórmula:

$$
H^{\prime}=-\sum_{i=1}^{S} p_{i} \cdot \ln p_{i}
$$

donde $p_{i}$ es la proporción del taxón iésimo en la muestra y $S$ la riqueza (número total de taxones) (Begon et al. 2006).

Los datos de densidad se transformaron por la función log $(x+1)$ y los taxones presentes sólo en una muestra no se consideraron (Clarke y Warwick 2001). Las diferencias en la composición de los ensambles según el factor "uso del suelo" (hortícola y referencia) fueron analizadas con ANOSIM (análisis de similaridad) 
utilizando el índice de similaridad de Bray Curtis. ANOSIM realiza un número de permutaciones posibles y proporciona un valor de probabilidad para establecer diferencias significativas entre los sitios y un estadístico R (Clarke y Warwick 2001); este valor cercano a 1 indica que los sitios son diferentes entre sí, mientras que un $\mathrm{R}$ cercano a 0 indica que los sitios son semejantes, es indicativo del grado de separación entre los sitios. En base a la matriz de similitud entre muestras se realizó un análisis de agrupamiento de cluster que agrupó los muestreos en función de la composición de taxones. Se realizó en análisis SIMPER (similaridad porcentual) para determinar qué taxones contribuyeron más a las diferencias entre los ensambles. Estos análisis se realizaron con el programa PRIMER (Plymouth Routines Multivariate Ecological Research, versión 6) (Clarke and Gorley, 2001).

Se determinó la frecuencia de detección de plaguicidas para cada arroyo muestreado de la siguiente manera:

$$
\text { Frecuencia de detección }=\frac{\mathrm{N}^{\circ} \text { de muestras con determinaciones }}{\mathrm{N}^{\circ} \text { de muestreos }}
$$

Las diferencias entre sitios hortícolas y de referencia con respecto a las variables ambientales, nutrientes, frecuencia de detección de plaguicidas y métricas de invertebrados se testearon a través del Test de T-Student. Cuando los datos no presentaron una distribución normal se utilizó el test de $U$ de Mann Whitney. Los análisis estadísticos se realizaron con SigmaStat 3.5 con un nivel de significancia de $p<0,05$.

Las relaciones entre las métricas y concentraciones de nutrientes, clorofila, plaguicidas, cobertura de la vegetación y porcentaje de horticultura en la cuenca se exploraron mediante el análisis de correlaciones de Pearson con SigmaStat 3.5 a un nivel de significancia de $p<0,05$.

Las relaciones entre las variables ambientales y la composición del ensamble se analizaron mediante el programa CANOCO versión 4.53 (ter Braak and Smilauer, 1998). Los taxones con abundancia relativa total menor a $0,5 \%$ se excluyeron del análisis para reducir la influencia de especies raras (Clarke and Warwick 2001). La máxima longitud del gradiente de los datos de invertebrados se determinó mediante el análisis de correspondencia detendenciado (DCA, Detrended Correspondence Analysis), siendo 1,8 para el primer periodo y 2,55 para el segundo, indicando en ambos periodos un comportamiento de los datos lineal (menor a 3) (Leps y Smilauer 2003). Consecuentemente, se realizó un Análisis de Redundancia (RDA, Redundancy 
Analysis). Los datos fueron normalizados y se realizó una selección (forward selection) para determinar aquellas variables que explicaron significativamente el patrón de distribución de los invertebrados, en un nivel de significancia de $p<0,05$. Para remover el efecto de la vegetación, se realizó posteriormente un análisis de redundancia parcial o particionado ( $\mathrm{p}-\mathrm{RDA}$, Partial Redundancy Analysis) utilizando la cobertura de la vegetación como covariable. La significancia de los ejes del RDA se testeó mediante el test de Monte Carlo, basado en 499 permutaciones.

Se analizó la influencia de la vegetación en la composición de los ensambles, se compararon las métricas entre los pares de sitios que presentaron igual tipo de vegetación mediante el test de T-Student. Cuando los datos no presentaron una distribución normal se utilizó el test de $U$ de Mann Whitney. Para evaluar la composición de los ensambles según la vegetación y el uso, se realizó un ANOSIM de dos vías con los factores "tipo de vegetación" y "uso del suelo" en base al índice de similaridad de Bray Curtis. Para disminuir la variabilidad de los valores de densidad de los taxones se utilizaron los datos de densidad a nivel de orden de los invertebrados de ambos periodos y se transformaron por la función log $(x+1)$. Estos análisis se realizaron con PRIMER.

\section{Resultados}

\section{Análisis espacial}

Se determinó la superficie en hectáreas de las parcelas ocupadas con horticultura en cada cuenca (tabla 3.1). El Arroyo del Gato presentó la mayor área ocupada por parcelas hortícolas seguido del $\mathrm{A}^{\circ}$ El Sauce, ocupando en ambos un área de aproximadamente 600 ha, seguidos de $A^{\circ}$ Martín con aproximadamente 460 ha y San Juan con 350 ha.

Se calculó el porcentaje ocupado por horticultura para cada una de las cuencas. El mayor porcentaje de ocupación con horticultura fue en el $A^{\circ}$ San Juan con $23 \%$ seguido por el Sauce con 17\%, Martín y Del Gato contaron con $14 \%$ de sus cuencas destinadas a la horticultura. El mapa generado se muestra en la figura 3.4. Se comprobó que los arroyos Sin Nombre, Buñirigo, Morales y Destino no presentaron parcelas con horticultura, por lo que el porcentaje de horticultura en dichas cuencas se determinó como $0 \%$, pudiendo considerarlos en conjunto como sitios de referencia. 
Tabla 3.1. Áreas con horticultura en las cuencas estudiadas.

\begin{tabular}{cccc}
\hline Arroyo & $\begin{array}{c}\text { Área de la } \\
\text { cuenca (ha) }\end{array}$ & $\begin{array}{c}\text { Parcelas con } \\
\text { Horticultura (ha) }\end{array}$ & \% Ocupado \\
\hline San Juan & 1549,8 & 352,8 & 22,8 \\
Martín & 3377,8 & 458,3 & 13,6 \\
Del Gato & 4314,5 & 601,7 & 13,9 \\
Del Sauce & 3456,4 & 583,9 & 16,9 \\
\hline
\end{tabular}
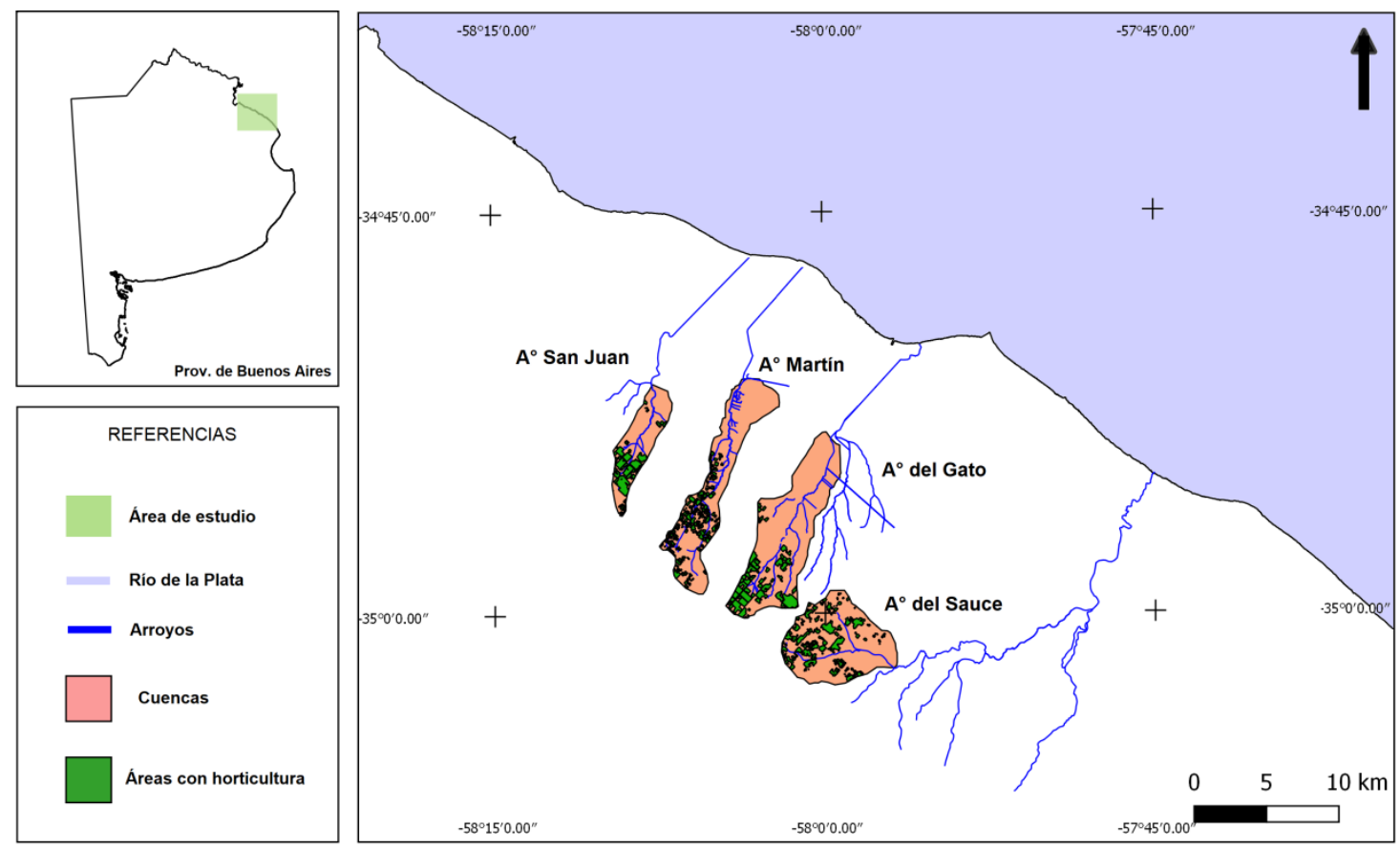

Figura 3.4. Mapa de los arroyos estudiados, delimitación de cuencas y área ocupada por horticultura intensiva.

\section{Muestreos}

El primer periodo comprendió 7 muestreos, en Octubre y Diciembre de 2014, y en Enero, Marzo, Mayo, Octubre y Diciembre de 2015. Luego comenzó un periodo de sequía que impidió continuar con los muestreos de la temporada de verano. La segunda etapa comprendió los meses de Noviembre y Diciembre de 2016, Enero y Marzo de 2017. En el segundo periodo el arroyo San Juan se encontró seco en todas las fechas de muestreo (tabla 3.2). 
Tabla 3.2. Muestreos realizados (blancos) y no realizados (grises) en ambos periodos

\begin{tabular}{|c|c|c|c|c|c|c|c|c|c|c|c|}
\hline \multirow[b]{2}{*}{ Fecha } & \multicolumn{7}{|c|}{ Primer etapa } & \multicolumn{4}{|c|}{ Segunda etapa } \\
\hline & $\begin{array}{c}\text { oct- } \\
14\end{array}$ & $\begin{array}{c}\text { dic- } \\
14\end{array}$ & $\begin{array}{c}\text { ene- } \\
15\end{array}$ & $\begin{array}{c}\text { mar- } \\
15\end{array}$ & $\begin{array}{c}\text { may- } \\
15\end{array}$ & $\begin{array}{c}\text { oct- } \\
15\end{array}$ & $\begin{array}{c}\text { dic- } \\
15\end{array}$ & $\begin{array}{c}\text { nov- } \\
16\end{array}$ & $\begin{array}{c}\text { dic- } \\
16\end{array}$ & $\begin{array}{c}\text { ene- } \\
17\end{array}$ & $\begin{array}{c}\text { mar- } \\
17\end{array}$ \\
\hline Número & 1 & 2 & 3 & 4 & 5 & 6 & 7 & 8 & 9 & 10 & 11 \\
\hline \multicolumn{12}{|l|}{ San Juan (SJ) } \\
\hline \multicolumn{12}{|l|}{ Martín (MR) } \\
\hline \multicolumn{12}{|l|}{ Gato (GA) } \\
\hline \multicolumn{12}{|l|}{ El Sauce (ES) } \\
\hline \multicolumn{12}{|l|}{ Sin Nombre (SN) } \\
\hline \multicolumn{12}{|l|}{ Buñirigo (BU) } \\
\hline \multicolumn{12}{|l|}{ Morales (MO) } \\
\hline Destino (DE) & & & & & & & & & & & \\
\hline
\end{tabular}

Se analizaron los valores de precipitación media mensual de los meses comprendidos en el total del periodo de muestreo. Para el año 2014, la sumatoria de las precipitaciones Octubre-Noviembre-Diciembre fue de $413 \mathrm{~mm}$, mientras que este mismo periodo para el año 2015 fue aproximadamente la mitad que el periodo anterior (242 mm). Para el año 2016 fue $313 \mathrm{~mm}$.

\section{Primer periodo: Octubre 2014-Diciembre 2015}

\section{Variables ambientales}

Las variables ambientales y las concentraciones de nutrientes se resumen en la tabla 3.3. Las concentraciones de nitrato, amonio y fósforo reactivo soluble (PRS) fueron significativamente mayores en los arroyos hortícolas en comparación con los arroyos de referencia ( $p=<0,001 ; p=0,002 ; p=<0,001$ respectivamente). Las concentraciones medias de nitrato fueron un orden de magnitud mayor mientras que amonio y PRS fueron tres veces mayores en los arroyos hortícolas. 
Tabla 3.3 Variables ambientales y concentración de nutrientes en arroyos. Valores medios y (rangos de variación).

\begin{tabular}{|c|c|c|}
\hline Variables & Horticultura & Referencia \\
\hline $\mathrm{T}^{\circ}\left({ }^{\circ} \mathrm{C}\right)$ & $19,8(12-30)$ & $22,3(12-29)$ \\
\hline Conductividad (Cond.) ( $\mu \mathrm{S} / \mathrm{cm})$ & $502(110-853)$ & $687(83-1330)$ \\
\hline Oxígeno Disuelto (OD) (mg/L) & $6,7(1,3-19)$ & $6,8(2,5-10,6)$ \\
\hline $\mathrm{pH}$ & $7,7(6,6-9,3)$ & $7,8(6,9-9,2)$ \\
\hline Profundidad media (cm) & $23(5-50)$ & $34(11-63)$ \\
\hline Sólidos suspendidos (SS) (mg/L) & $103(2-1208)$ & $105(13-457)$ \\
\hline Carbono orgánico del sedimento (COrg\%) & $3,9(1-7)$ & $3,7(1-9)$ \\
\hline Clorofila ( $\mu \mathrm{g} / \mathrm{L})$ & $45(4-355)$ & $24(3,5-82)$ \\
\hline Cobertura de la vegetación (Veg) (\%) & $62(15-100)$ & $58(17-90)$ \\
\hline Fósforo Reactivo Soluble (PRS) ( $\mu \mathrm{g} / \mathrm{L})$ & $319(84-765)$ & $109(16-434)$ \\
\hline Nitrito ( $\mu \mathrm{g} \mathrm{N}-\mathrm{NO2} / \mathrm{L}$ ) & $61(7-247)$ & $17(1-180)$ \\
\hline Nitrato ( $\left.\mu \mathrm{g} \mathrm{N}-\mathrm{NO}_{3}{ }^{-} / \mathrm{L}\right)$ & $1183(20-6808)$ & $95(5-367)$ \\
\hline Amonio ( $\left.\mu \mathrm{g} \mathrm{N}-\mathrm{NH}_{4}{ }^{+} / \mathrm{L}\right)$ & $229(16-1334)$ & $71(4-414)$ \\
\hline
\end{tabular}

\section{Plaguicidas}

En general no se detectaron insecticidas en los arroyos de referencia, a excepción de clorpirifos en arroyos de la reserva y en Buñirigo en bajas concentraciones (1,5 a 5,8 ng/g peso seco) en Diciembre 2014, y cipermetrina en Buñirigo y Sin Nombre (10-45 ng/g-p.s.) en Enero 2015 (tabla 3.4)

Se detectó cipermetrina en 5 ocasiones en los arroyos hortícolas, alcanzando altas concentraciones de 148-199 ng/g ps en dos muestreos sucesivos en el arroyo del Gato y $51 \mathrm{ng} / \mathrm{g}$ p.s. en el arroyo del Sauce. En los arroyos hortícolas también se detectaron clorpirifos, lambdacialotrina, $p, p^{\prime}-D D T$ y $p^{\prime} p-D D D$ en bajas concentraciones (0,8-4 ng/g p.s.). La frecuencia de detección de plaguicidas fue significativamente mayor en los arroyos hortícolas que los de referencia $(p=0,023)$.

\section{Ensamble de invertebrados}

Se contabilizaron un total de 30829 especímenes y se identificaron 88 taxones, representando 52 familias y 18 órdenes (tabla 3.5). Las especies del género Simocephalus y el anfípodo Hyalella sp. fueron los más abundantes en los sitios de referencia, excepto en Sin Nombre donde, además de los cladóceros, el grupo dominante fue Copepoda. Decapoda sólo estuvo presente en los sitios de referencia. Se identificaron dos familias de Trichoptera: Hydroptilidae y Polycentropodidae (Cyrnellus sp.) que estuvieron presentes sólo en arroyos de referencia Buñirigo y 
Destino, y ausentes en sitios hortícolas. Ephemeroptera presentó altas densidades en Buñirigo y Destino, Baetidae fue raro y Caenis estuvo ausente en sitios hortícolas. Por el contrario, los colémbolos (Entomobryoidea y Poduroidea) fueron los taxones más abundantes en los arroyos hortícolas; otros taxones dominantes fueron Diptera, Copepoda y Dugessidae, este último raro en los arroyos de referencia.

Tabla 3.5. Densidad media (ind $/ \mathrm{m}^{2}$ ) de los taxones identificados

\begin{tabular}{|c|c|c|c|c|c|c|c|c|c|c|}
\hline \multirow[b]{2}{*}{ GRUPO } & \multirow[b]{2}{*}{ FAMILIA } & \multirow[b]{2}{*}{ TAXONES } & \multicolumn{4}{|c|}{ HORTÍCOLAS } & \multicolumn{4}{|c|}{ REFERENCIA } \\
\hline & & & San Juan & Martín & Gato & Sauce & $\begin{array}{c}\text { Sin } \\
\text { Nombre }\end{array}$ & Buñirigo & Morales & Destino \\
\hline \multicolumn{11}{|l|}{ COLLEMBOLA } \\
\hline & Entomobryoidea & Entomobryoidea & 75 & 103 & 59 & 36 & 24 & 10 & 4 & 14 \\
\hline & Poduroidea & Poduroidea & 18 & 7 & 1 & 12 & & & 2 & \\
\hline & Sminthuroidea & Sminthuroidea & 2 & 2 & 23 & 2 & 5 & 12 & 5 & 6 \\
\hline \multicolumn{11}{|l|}{ INSECTA } \\
\hline \multirow[t]{2}{*}{ Ephemeroptera } & Baetidae & Baetidae & & & & 1 & 3 & 50 & 7 & 19 \\
\hline & Caenidae & Caenis & & & & & 5 & 25 & & 11 \\
\hline \multirow[t]{3}{*}{ Odonata } & Aeshnidae & Aeshnidae & 1 & & & & & & 1 & \\
\hline & Coenagrionidae & Coenagrionidae & 40 & 2 & 17 & 3 & 42 & 10 & 29 & 32 \\
\hline & Libellulidae & Libellulidae & & & & & 1 & & & 1 \\
\hline \multirow[t]{8}{*}{ Heteroptera } & Belostomatidae & Belostoma & 1 & & 1 & 2 & 5 & & 16 & 4 \\
\hline & Corixidae & Corixidae (L) & & & 1 & & 9 & 1 & 2 & 3 \\
\hline & & Sigara & 0 & & & & 4 & & 1 & 1 \\
\hline & Hebridae & Lipogomphus & & & 1 & & & & 4 & 1 \\
\hline & Mesoveliidae & Mesoveliidae & 2 & & 1 & 1 & & & 1 & 2 \\
\hline & Notonectidae & Notonecta & & & & & 15 & & 2 & \\
\hline & Pleidae & Neoplea & & & & & 2 & & 5 & 2 \\
\hline & Veliidae & Microvelia & 1 & 1 & 3 & 1 & 2 & & & \\
\hline Trichoptera & Hydroptilidae & Hydroptilidae & & & & & & 3 & & \\
\hline \multirow[t]{8}{*}{ Diptera } & Ceratopogonidae & Ceratopogonidae & 4 & & & & & 13 & 1 & 3 \\
\hline & Chironomidae & Chironomidae & 68 & 2 & 5 & 1 & 2 & 11 & 30 & 15 \\
\hline & Culicidae & Culicidae & & & & & & & 1 & 1 \\
\hline & Ephydridae & Ephydridae & 195 & & 1 & 1 & & & 1 & 14 \\
\hline & Psycodidae & Psycodidae & 1 & & & & & & & \\
\hline & Sciomyzidae & Sciomyzidae & & & & & & & & 1 \\
\hline & Stratiomyidae & Stratiomyidae & 27 & & 5 & & 1 & 1 & 1 & 3 \\
\hline & Tipulidae & Tipulidae & 1 & & & & & & & 1 \\
\hline
\end{tabular}


Diptera undet.

\begin{tabular}{|c|c|c|c|c|c|c|c|c|c|c|}
\hline \multirow[t]{16}{*}{ Coleoptera } & Curculionidae & Curculionidae & 2 & & 1 & 1 & 2 & & 60 & 3 \\
\hline & Dytiscidae & Desmopachria & & & 1 & & & & & \\
\hline & & Dytiscidae (L) & & & & & 10 & & 3 & 1 \\
\hline & & Laccophilus & & & & & 8 & & 3 & 1 \\
\hline & & Liodessus & 7 & 1 & 4 & 1 & 4 & 2 & 1 & 1 \\
\hline & Elmidae & Elmidae & 1 & & & & & 1 & & \\
\hline & Hydrophilidae & Berossus & & & & & 1 & 5 & 3 & \\
\hline & & Derallus & 2 & 1 & 2 & 1 & 2 & 1 & & 1 \\
\hline & & Enochrus & 23 & 2 & 3 & 2 & 2 & 2 & 4 & 3 \\
\hline & & Paracymus & 3 & 2 & 4 & 2 & 5 & 5 & 1 & 9 \\
\hline & & Tropisternus & & & 1 & & 3 & 1 & 17 & 5 \\
\hline & Noteridae & Hydrocanthus & & & & & 2 & & 3 & 2 \\
\hline & & Noteridae (L) & & & & & & & 1 & \\
\hline & & Suphis & & & & & & & 1 & \\
\hline & & Suphisellus & & & & & 3 & & 1 & 1 \\
\hline & Scirtidae & Scirtidae & 3 & & 2 & 2 & & & & \\
\hline \multicolumn{11}{|c|}{ CRUSTACEA } \\
\hline Amphipoda & Hyalellidae & Hyalella & 33 & 9 & 46 & 6 & 47 & 119 & 880 & 264 \\
\hline \multirow[t]{6}{*}{ Cladocera } & Chydoridae & Chydoridae & & & & & 4 & & 2 & \\
\hline & Daphnidae & Ceriodaphnia & & & & & 7 & 1 & & \\
\hline & & S. daphnoides & 19 & & 1 & 3 & 48 & 94 & 12 & 114 \\
\hline & & S. vetulus & 9 & & 13 & 27 & 61 & 181 & 50 & 193 \\
\hline & Sididae & Diaphanosoma & & & 1 & & 31 & 2 & 29 & 16 \\
\hline & & Cladocera undet. & & & & & & & 1 & \\
\hline \multirow[t]{2}{*}{ Copepoda } & Calanoidea & Calanoidea & & & & & 122 & & & 1 \\
\hline & Ciclopoidea & Ciclopoidea & 106 & 5 & 171 & 10 & 124 & 33 & 15 & 11 \\
\hline Decapoda & Palaeomonidae & Palaeomonetes & & & & & & 4 & & \\
\hline \multirow[t]{3}{*}{ Ostracoda } & Cyprididae & C. ihieringi & & & & & 3 & & & \\
\hline & & C. incisa & & & 5 & & 2 & & 1 & 3 \\
\hline & & Chlamydotheca & 1 & 1 & & & 4 & 3 & & \\
\hline
\end{tabular}

ARACHNIDA

Acari

Acari

ANNELIDA

Hirudinea

Hirudinea

121

Oligochaeta

19

4
17

$21 \quad 2$

$5 \quad 11$

2

$\begin{array}{ccc}1 & 46 & 4 \\ 6 & 6 & 12\end{array}$

MOLLUSCA 


\begin{tabular}{|c|c|c|c|c|c|c|c|c|c|}
\hline Bivalvia & Sphaeriidae & Sphaeriidae & & & 1 & 5 & 3 & & \\
\hline \multirow[t]{6}{*}{ Gasteropoda } & Ampullaridae & Pomacea & & 4 & 4 & 3 & 4 & 7 & 6 \\
\hline & Ancylidae & Uncancylus & 2 & & & 1 & 28 & 3 & 2 \\
\hline & Cochliopidae & Heleobia & & & & 4 & & & \\
\hline & Physidae & Physidae & & 1 & 13 & & 1 & & \\
\hline & Planorbidae & Biomphalaria & & 2 & 1 & & 1 & & 5 \\
\hline & & Drepanotrema & 14 & 0 & 11 & 6 & 48 & & 15 \\
\hline
\end{tabular}

\section{PLATHELMYNTHES}

Tricladida

Dugesiidae

Dugesiidae

154

3

$\begin{array}{llll}39 & 10 & 3 & 1\end{array}$

Los taxones con densidad media menor a la unidad no se muestran. (L) indica estado juvenil o larva. Los nombres completos de las especies mencionadas se encuentran en el Anexo.

La riqueza de taxones y la diversidad medias fueron significativamente mayores en los sitios de referencia (35 taxones y $819 \mathrm{ind} / \mathrm{m}^{2}$ ) en comparación con los sitios hortícolas (24 taxones y $461 \mathrm{ind} / \mathrm{m}^{2}, \mathrm{p}=<0,001$ y $\mathrm{p}=0,025$ respectivamente) (tabla 3.6). Sin Nombre fue el arroyo con mayor riqueza taxonómica (41 taxones registrados) y Morales fue el arroyo con mayor densidad (1286 ind $\left./ \mathrm{m}^{2}\right)$, mientras que el arroyo Martín, el que registró la mayor frecuencia de detección de plaguicidas y mayores concentraciones de PRS, resultó el más pobre con 18 taxones y $172 \mathrm{ind} / \mathrm{m}^{2}$. Las densidades de los crustáceos Hyalella sp. $\left(239 \mathrm{ind} / \mathrm{m}^{2}\right)$ y $S$. vetulus $\left(136 \mathrm{ind} / \mathrm{m}^{2}\right)$ fueron significativamente mayores en los sitios de referencia en comparación con los hortícolas (25 y 13 ind $/ \mathrm{m}^{2}, \mathrm{p}=0,04$ and $\mathrm{p}=<0,001$ respectivamente). Los arroyos más diversos fueron Sin Nombre, El Sauce y Destino, los menos diversos Martín y Morales, no hubo diferencias significativas en la diversidad entre arroyos hortícolas y de referencia $(p=0,665)$.

Tabla 3.6. Métricas promedio por arroyo

\begin{tabular}{ccccc|cccc} 
& \multicolumn{3}{c|}{ HORTÍCOLAS } & \multicolumn{3}{c}{ REFERENCIA } \\
\cline { 2 - 9 } & $\begin{array}{c}\text { San } \\
\text { Juan }\end{array}$ & Martín & Gato & $\begin{array}{c}\text { El } \\
\text { Sauce }\end{array}$ & $\begin{array}{c}\text { Sin } \\
\text { Nombre }\end{array}$ & Buñirigo & Morales & Destino \\
\hline $\begin{array}{c}\text { Riqueza } \\
\text { Densidad } \\
\text { (ind/m }{ }^{2} \text { ) }\end{array}$ & 23 & 18 & 31 & 22 & 41 & 30 & 35 & 35 \\
Diversidad (H') & 1,86 & 1,67 & 1,94 & 2,21 & 2,41 & 1,89 & 1,67 & 2,07 \\
\hline
\end{tabular}


El análisis cluster mostró tres agrupamientos de los sitios (fig. 3.5). El grupo A conformado en su totalidad por muestras de sitios de referencia, y los grupos $\mathrm{B}$ y $\mathrm{C}$ conformados por muestras de arroyos hortícolas: el grupo B presenta 21 muestras de las cuales 20 son de arroyos hortícolas y una muestra pertenece a Sin Nombre (Octubre 2014), mientras que el grupo C agrupó muestreos de los arroyos El Sauce, Martín y un muestreo de Buñirigo (Enero 2015).

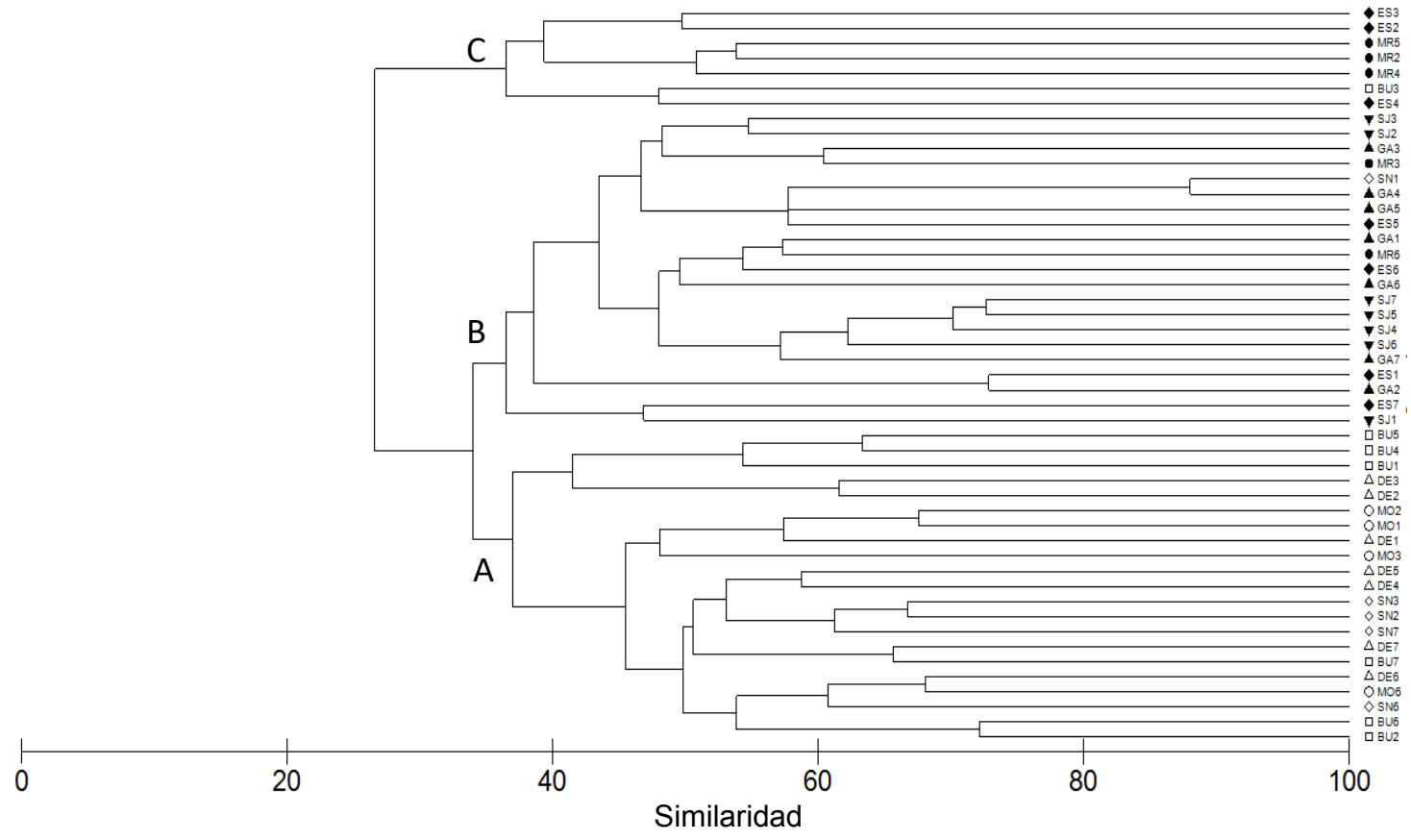

Fig 3.5. CLUSTER de los sitios muestreados durante el primer periodo en base a matriz de similitud con Bray Curtis. Símbolos negros: sitios hortícolas. Símbolos blancos: sitios de referencia. SJ: San Juan, MR: Martín, ES: El Sauce, AG: del Gato, SN: Sin Nombre, BU: Buñirigo, MO: Morales y DE: Destino. 1: Oct-2014, 2: Dic-2014, 3: Ene-2015, 4: Mar-2015, 5: May-2015, 6: Oct-2015, 7: Dic-2015.

La composición del ensamble en arroyos hortícolas y de referencia fue significativamente diferente (ANOSIM: $R$ Global $=0,45$ y $p=0,001$ ).El análisis SIMPER (tabla 3.7) mostró una disimilaridad promedio de $68 \%$ entre ambos grupos. Los taxones que más contribuyeron a estas diferencias fueron $S$. vetulus, Hyalella sp. y Baetidae, bien representados en arroyos de referencia, y Caenis debido a su ausencia en arroyos hortícolas. Dugessidae, Entomobryoidea e Hirudinea contribuyeron a las diferencias debido a su comparativamente mayor abundancia en sitios hortícolas. 
Tabla 3.7. Análisis SIMPER mostrando abundancias medias (transformadas por logaritmo) de los taxones y su contribución $>30 \%$ de la disimilaridad.

\begin{tabular}{|c|c|c|c|c|}
\hline \multirow{2}{*}{ Taxones } & \multicolumn{2}{|c|}{ Abundancia media } & \multirow{2}{*}{$\begin{array}{c}\text { Contribución } \\
(\%)\end{array}$} & \multirow{2}{*}{$\begin{array}{c}\text { Contr. } \\
\text { Acum. (\%) }\end{array}$} \\
\hline & Horticultura & Referencia & & \\
\hline S. vetulus & 1,1 & 3,6 & 5,0 & 5,0 \\
\hline Hyalella & 1,9 & 3,8 & 4,7 & 9,7 \\
\hline Baetidae & 0,1 & 2,2 & 4,0 & 13,7 \\
\hline Dugesiidae & 2,5 & 0,3 & 3,8 & 17,6 \\
\hline S. daphnoides & 0,6 & 2,5 & 3,8 & 21,3 \\
\hline Ciclopoidea & 2,0 & 2,5 & 3,5 & 24,9 \\
\hline Entomobryoidea & 3,3 & 1,9 & 3,4 & 28,2 \\
\hline Caenis & 0,0 & 1,7 & 3,2 & 31,4 \\
\hline Hirudinea & 2,0 & 1,0 & 2,9 & 34,3 \\
\hline
\end{tabular}

\section{Correlaciones}

Las concentraciones de nutrientes se relacionaron positivamente con el porcentaje de la cuenca ocupado por horticultura (PRS: $R=0,54$ y $p<0,0001$; nitrito: $R=0,44$ y $p=0,001$; nitrato: $R=0,44$ y $p=0,0015$; amonio: $R=0,45$ y $p=0,0013$ ). La concentración de clorofila presentó una correlación positiva con PRS $(R=0,28$; $p=0,049)$ y amonio $(R=0,28 ; p=0,048)$.

La riqueza de taxones y la densidad de invertebrados estuvo positivamente relacionada con la cobertura de la vegetación $(R=0,35$ y $p=0,015 ; R=0,45$ y $p=0,001$, respectivamente). La riqueza estuvo inversamente relacionada al $P R S(R=-0,33$ y $p=0,021)$ y al amonio ( $R=-0,32$ y $p=0,024)$, mientras que la densidad estuvo inversamente relacionada con las concentraciones de PRS ( $R=-0,42$ y $p=0,002)$. La riqueza se relacionó negativamente con el porcentaje de la cuenca ocupado por horticultura $(R=-0,53$ y $p<0,0001)$.

Se analizó la relación de los taxones más representativos de los ensambles obtenidos con el método SIMPER con el porcentaje de horticultura en la cuenca. $S$. vetulus, Hyalella, Baetidae y Caenis se correlacionaron negativamente con el porcentaje de horticultura mientras que Entomobryoidea, Dugessidae e Hirudinea se correlacionaron positivamente. Los valores de significancia y del coeficiente de correlación se muestran en la siguiente tabla (3.8). 
Tabla 3.8. Correlaciones entre taxones y porcentaje ocupado por horticultura en la cuenca. En negritas se muestran los valores significativos $(p<0,05)$.

\begin{tabular}{ccc}
\hline Taxones & $\mathrm{R}$ & $\mathrm{p}$ \\
\hline S. vetulus & $-0,362$ & $\mathbf{0 , 0 1}$ \\
Hyalella & $-0,339$ & $\mathbf{0 , 0 2}$ \\
Baetidae & $-0,462$ & $\mathbf{0 , 0 0 1}$ \\
Caenis & $-0,39$ & $\mathbf{0 , 0 0 6}$ \\
Entomobryoidea & 0,311 & $\mathbf{0 , 0 3}$ \\
Dugesiidae & 0,487 & $\mathbf{0 , 0 0 0 4}$ \\
Hirudinea & 0,316 & $\mathbf{0 , 0 3}$ \\
\hline
\end{tabular}

\section{Análisis multivariados}

El análisis de redundancia indicó que la cobertura de la vegetación, el fósforo reactivo soluble, oxígeno disuelto, amonio y carbono orgánico explicaron el $32 \%$ de la variabilidad total en el ensamble de invertebrados. La significancia global para el primer eje canónico y para la suma de todos los ejes fue significativo ( $F=6,768$ y $p=0,002 ; F=2,260$ y $p=0,002$; respectivamente). Como la vegetación influyó en la riqueza y la abundancia de los taxones del ensamble, se consideró separadamente; se realizó un análisis parcial utilizando la vegetación como covariable (Fig.3.8). Este RDA explicó el $23 \%$ de la variación remanente. El primer y el segundo eje explicaron el $75 \%$ de la relación entre los taxones y el ambiente. El primer eje se correlacionó con PRS $(0,6)$, amonio $(\mathrm{NH} 4)(0,41)$ y porcentaje de carbono orgánico en el sedimento (Corg\%) $(0,04)$, representando un gradiente de uso de suelo. El segundo eje estuvo positivamente relacionado con oxígeno disuelto $(\mathrm{OD})(0,66)$ y $\mathrm{C}$ org\% $(0,06)$, y negativamente relacionado a la conductividad (Conduct.) $(-0,33)$.

Este análisis mostró una correspondencia entre la abundancia de ciertos taxones de invertebrados y los gradientes (Fig 3.6, izquierda). Entomobryoidea, Poduroidea, Hirudinea, Dugessidae, Paracymus sp., Pomacea sp. y Enochrus sp. se correlacionaron con el uso de suelo hortícola, mientras que Hyalella sp., las especies de Simocephalus, Caenis sp., Baetidae, Ceratopogonidae y Tropisternus sp. estuvieron negativamente relacionados. En diagrama de sitios de muestreo (Fig. 3.6, derecha), las muestras de sitios de referencia se localizaron en el sector izquierdo del diagrama de ordenamiento y casi todos los muestreos de sitios hortícolas a la derecha del diagrama, relacionados a mayores concentraciones de fósforo y amonio, reflejando el gradiente de uso del suelo a lo largo del primer eje. 

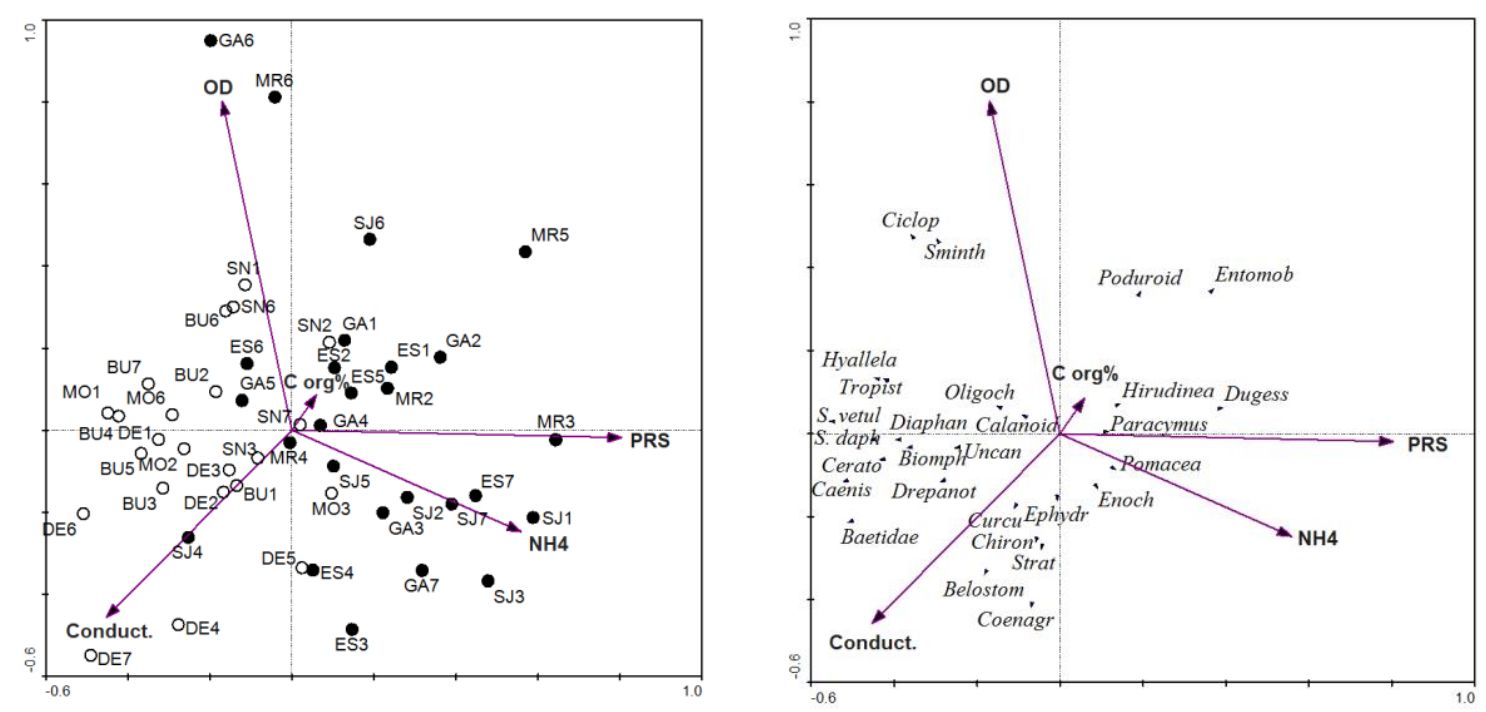

Fig. 3.6. Análisis de Redundancia Parcial. Gráficos de datos ambientales y especies (izquierda) y sitios (derecha). Círculos blancos: sitios de referencia; círculos negros: sitios hortícolas. SJ: San Juan, MR: Martín, GA: Gato, ES: Sauce, SN: Sin Nombre, BU: Buñirigo, MO: Morales, DE: Destino. 1: Oct-2014, 2: Dic-2014, 3: Ene-2015, 4: Mar-2015, 5: May-2015, 6: Oct-2015, 7: Dic-2015. Se muestran solo las variables ambientales que explicaron significativamente el ensamble de invertebrados.

\section{Segundo periodo: Noviembre 2016 - Marzo 2017}

\section{Variables ambientales}

Las variables ambientales y las concentraciones de nutrientes se resumen en la tabla 3.8. En general se mantuvieron las características observadas en el periodo anterior: conductividades alrededor de $500 \mu \mathrm{s} / \mathrm{cm}, \mathrm{pH}$ de neutros a alcalinos y la cobertura vegetal mayor al $50 \%$ en casi todos los sitios. Se encontraron diferencias significativas en la temperatura $(p=0,026), p H(p=0,035)$ y sólidos suspendidos $(p=0,049)$, siendo los valores medios de estas variables menores en los sitios hortícolas que en los sitios de referencia. Las concentraciones de nitrato, amonio y PRS fueron significativamente mayores en los arroyos hortícolas en comparación con los arroyos de referencia ( $p=0,002 ; p=0,007 ; p=<0,001$ respectivamente). Las concentraciones medias de nitrato y PRS en los arroyos hortícolas mostraron valores entre 100 y $500 \mu \mathrm{g} / \mathrm{L}$ siendo la mayor concentración de nitrato en el $A^{\circ}$ Martín (442 $\mu \mathrm{g} / \mathrm{L})$ y la mayor concentración de PRS en el Gato $(509 \mu \mathrm{g} / \mathrm{L}$, mientras que en arroyos de referencia las concentraciones medias de nitrato y PRS variaron entre 10 y 100 $\mu \mathrm{g} / \mathrm{L}$, con algunos valores extremos de nitrato en Destino y Morales que superaron los $100 \mu \mathrm{g} / \mathrm{L}$ y en Sin Nombre que superaron los $200 \mu \mathrm{g} / \mathrm{L}$. 
Tabla 3.8. Variables ambientales y concentración de nutrientes en arroyos. Valores medios y (rangos de variación).

\begin{tabular}{rcc}
\hline Variables & Horticultura & Referencia \\
\hline $\mathrm{T}^{\circ}\left({ }^{\circ} \mathrm{C}\right)$ & $20,4(15-32)$ & $23,6(19-34)$ \\
Conductividad (Cond.) $(\mu \mathrm{S} / \mathrm{cm})$ & $392(141-672)$ & $578(117-995)$ \\
Oxígeno Disuelto $(\mathrm{OD})(\mathrm{mg} / \mathrm{L})$ & $5,8(2,7-8)$ & $6,4(2,6-12)$ \\
$\mathrm{pH}$ & $7,6(7,3-8)$ & $8,1(7,2-8,8)$ \\
Profundidad $(\mathrm{cm})$ & $25,1(10-41,4)$ & $28,2(7-44,7)$ \\
Carbono orgánico del sedimento $(\mathrm{Corg} \%)$ & $4,5(2,2-10,7)$ & $3,3(1,4-6,5)$ \\
Clorofila $(\mu \mathrm{g} / \mathrm{L})$ & $33(25-83,3)$ & $27,3(27-100)$ \\
Cobertura de la vegetación $(\mathrm{VEG})(\%)$ & $63,2(25-83,3)$ & $70,4(27-100)$ \\
Fósforo Reactivo Soluble $(\mathrm{PRS})(\mu \mathrm{g} / \mathrm{L})$ & $290,2(63-509)$ & $93,2(21-237)$ \\
Nitrito $\left(\mu \mathrm{g} \mathrm{N}-\mathrm{NO}_{2}{ }^{-} / \mathrm{L}\right)$ & $22,6(4-68)$ & $12,2(1-39)$ \\
Nitrato $\left(\mu \mathrm{Ng}-\mathrm{NO}_{3} / \mathrm{L}\right)$ & $207,5(15-442)$ & $68,5(7-215)$ \\
Amonio $\left(\mu \mathrm{N} \mathrm{N}-\mathrm{NH}_{4}{ }^{+} / \mathrm{L}\right)$ & $66,7(34,7-126)$ & $45,7(3-89)$ \\
\hline
\end{tabular}

\section{Plaguicidas}

Se detectó clorpirifos en todas las muestras analizadas. Los arroyos hortícolas presentaron valores medios de 1550 (32-9127) ng/g p.s., correspondiendo las mayores concentraciones al Arroyo Martín (3340 y 9127 ng/g p.s.), mientras que en los arroyos de referencia se detectaron valores medios de 237 (de 6 a 1239) ng/g p.s. (tabla 3.9). El $89 \%$ de los sitios hortícolas presentaron al menos 2 plaguicidas detectados.

Se determinó $\alpha$ y $\beta$-endosulfán y su producto de degradación, endosulfán sulfato: en los arroyos de referencia con concentraciones $<0,1 \mathrm{ng}$ p.s., mientras que en los arroyos hortícolas se determinaron concentraciones entre 0,1-1,3 ng/g p.s., siendo la sumatoria de estos compuestos significativamente mayor en arroyos hortícolas $(p=0,003)$ comparado con los arroyos de referencia. Particularmente la frecuencia de detección de endosulfan sulfato fue significativamente mayor en los arroyos hortícolas (33-100 \%) que en los arroyos de los sitios de referencia $(0-33 \%)(p<0,05)$.

Asimismo, se detectó la presencia de DDT y sus productos de degradación DDE y DDD de 0,07 a 0,3 ng/g p.s. en arroyos hortícolas, y valores de $<0,1 \mathrm{ng} / \mathrm{g}$ en los arroyos de referencia, a excepción de una muestra de Destino $(0,15 \mathrm{ng} / \mathrm{g}$ p.s., enero). Como en este periodo en todas las muestras se detectó al menos un plaguicida, se calculó la frecuencia de detección como la proporción del total de plaguicidas 
detectados y el número de muestreos por arroyo (tabla 3.10). Los arroyos Gato y El Sauce presentaron un gran número de plaguicidas detectados (14 y 15 respectivamente) y el Gato también presentó la mayor frecuencia de detección de plaguicidas.

Tabla 3.9. Número de plaguicidas detectado por arroyo, número de muestras y frecuencia de detección.

\begin{tabular}{cccc|ccccc}
\cline { 2 - 8 } & \multicolumn{3}{c|}{ HORTíCOLAS } & \multicolumn{4}{c}{ REFERENCIA } \\
\cline { 2 - 8 } & Martín & El Gato & El Sauce & Sin Nombre & Buñirigo & Morales & Destino \\
\hline N plaguicidas detectados & 8 & 14 & 15 & 9 & 6 & 9 & 8 \\
N muestras & 3 & 2 & 4 & 3 & 4 & 3 & 4 \\
$\begin{array}{c}\text { Frecuencia de detección } \\
\text { de plaguicidas }\end{array}$ & 3 & 7 & 4 & 3 & 1,5 & 3 & 2 \\
\hline
\end{tabular}

\section{Ensamble de invertebrados}

Se contabilizaron un total de 15688 especímenes y se identificaron 82 taxones, representando 54 familias y 18 órdenes (tabla 3.11). El anfípodo Hyalella, el planórbido Drepanotrema y el cladócero $S$. vetulus fueron los más abundantes en los sitios de referencia. Los colémbolos fueron los taxones más abundantes en sitios hortícolas, seguido de Hyalella, C. incisa y Dugesiidae. Los efemeropteros Baetidae y Caenis y los odonatos de la familia Libellulidae fueron abundantes en los arroyos de referencia, los decápodos Palaeomonetes y Trichodactylidae, y los tricópteros Hydroptilidae y Cyrnellus se encontraron en sitios de referencia aunque en bajas densidades; todos estos taxones estuvieron ausentes en sitios hortícolas.

Tabla 3.10. Densidad media (ind $/ \mathrm{m}^{2}$ ) de los taxones identificados.

HORTICOLAS REFERENCIA

GRUPO

FAMILIA

TAXONES Martín El Gato El Sauce Sin Nombre

COLLEMBOLA

\begin{tabular}{|c|c|c|c|c|c|c|c|c|}
\hline Entomobryoidea & Entomobryoidea & 229 & 111 & 53 & 5 & 7 & 3 & 1 \\
\hline Poduroidea & Poduroidea & 5 & 2 & 11 & & & 2 & \\
\hline Sminthuroidea & Sminthuroidea & 2 & 2 & 1 & 4 & & 5 & 3 \\
\hline
\end{tabular}

INSECTA 


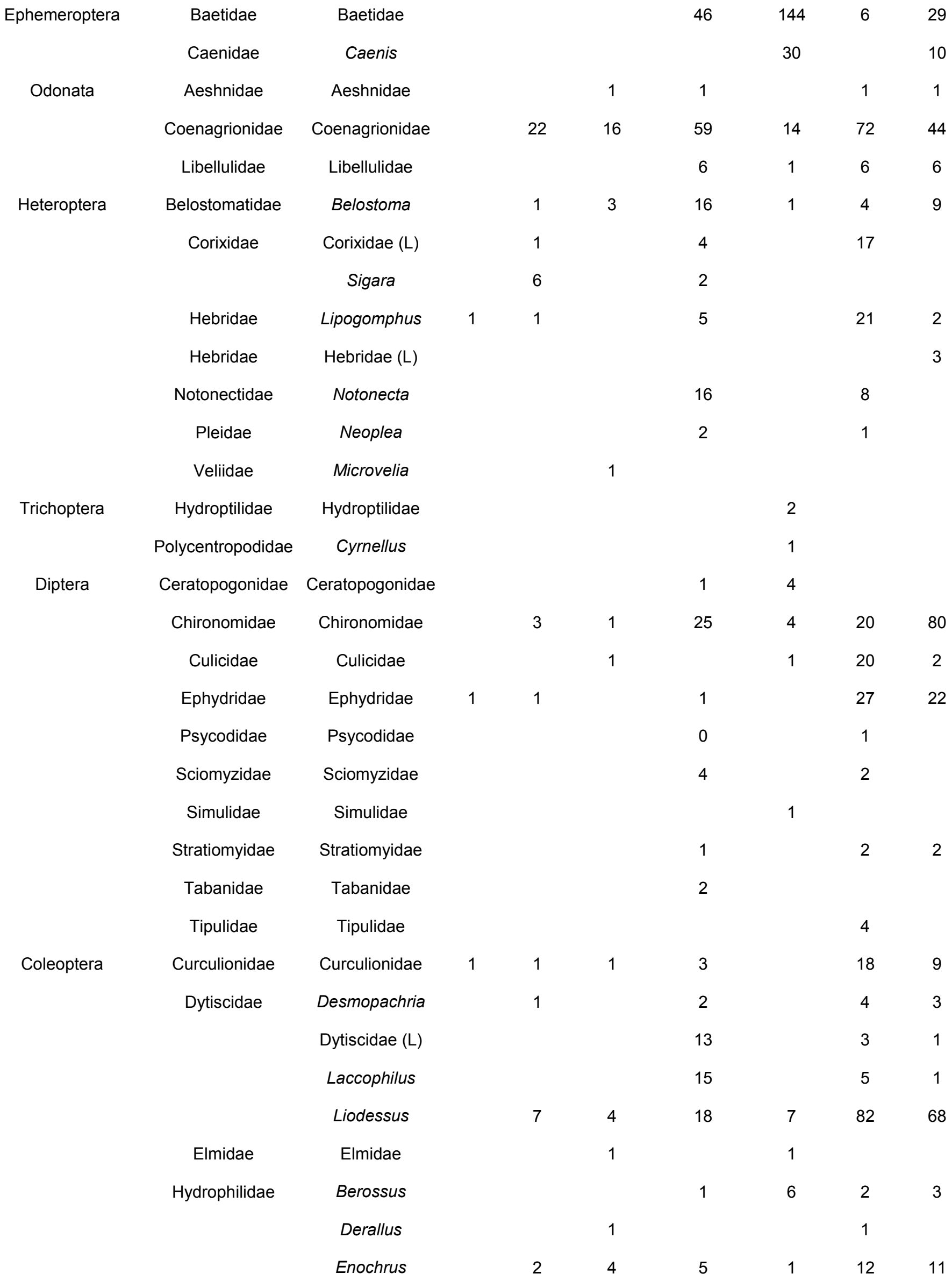




\begin{tabular}{|c|c|c|c|c|c|c|}
\hline & Paracymus & 4 & 3 & 9 & 3 & 13 \\
\hline & Tropisternus & & & 13 & 1 & 12 \\
\hline & Hydrophilidae (L) & & 1 & & & \\
\hline Noteridae & Noteridae (L) & & & & & 2 \\
\hline & Suphis & & & 1 & & \\
\hline & Suphisellus & & & 27 & & \\
\hline Scirtidae & Scirtidae & & 3 & & & \\
\hline & Coleoptera indet. & & & 1 & & \\
\hline
\end{tabular}

CRUSTACEA

\begin{tabular}{|c|c|c|c|c|c|c|c|c|c|}
\hline Amphipoda & Hyalellidae & Hyalella & 1 & 73 & & 152 & 167 & 172 & 92 \\
\hline \multirow[t]{5}{*}{ Cladocera } & Chydoridae & Chydoridae & & & & 3 & & 8 & \\
\hline & Daphnidae & Ceriodaphnia & & & & 18 & & & \\
\hline & & S. daphnoides & & 2 & & 66 & 11 & 1 & 114 \\
\hline & & S. vetulus & & 9 & 5 & 83 & 53 & 44 & 179 \\
\hline & Sididae & Diaphanosoma & & & & 37 & 6 & 41 & 12 \\
\hline \multirow[t]{2}{*}{ Copepoda } & Calanoidea & Calanoidea & & & & 7 & & 3 & \\
\hline & Ciclopoidea & Ciclopoidea & 1 & 10 & 5 & 30 & 10 & 43 & 37 \\
\hline \multirow[t]{2}{*}{ Decapoda } & Palaeomonidae & Palaeomonetes & & & & & 1 & & \\
\hline & Trichodactylidae & Trichodactylidae & & & & 1 & & & \\
\hline \multirow[t]{3}{*}{ Ostracoda } & Cyprididae & C. ihieringi & & & & 5 & & & 4 \\
\hline & & C. incisa & & 68 & & 11 & 1 & 9 & 1 \\
\hline & & Chlamydotheca & & 15 & & 2 & & & 1 \\
\hline \multicolumn{10}{|l|}{ ARACHNIDA } \\
\hline Acari & & Acari & & & 1 & 1 & & 26 & 1 \\
\hline \multicolumn{10}{|l|}{ ANNELIDA } \\
\hline Hirudinea & & Hirudinea & 1 & 8 & 3 & 1 & & 1 & \\
\hline Oligochaeta & & Oligochaeta & & 5 & 3 & 69 & 3 & 4 & 64 \\
\hline \multicolumn{10}{|l|}{ MOLLUSCA } \\
\hline Bivalvia & Sphaeriidae & Sphaeriidae & 10 & & 2 & & & & \\
\hline \multirow[t]{5}{*}{ Gasteropoda } & Ampullaridae & Pomacea & 5 & 1 & 8 & 3 & & & \\
\hline & Ancylidae & Uncancylus & & & 1 & 39 & & 2 & 17 \\
\hline & Cochliopidae & Heleobia & & & & & & & 3 \\
\hline & Physidae & Physidae & 3 & & 1 & & & & \\
\hline & Planorbidae & Biomphalaria & 2 & & 6 & 7 & & 8 & 36 \\
\hline
\end{tabular}




\section{PLATHELMYNTHES}

Los taxones con densidad media menor a la unidad no se muestran. (L) indica estado juvenil o larva. Los nombres completos de las especies mencionadas se encuentran en el

Anexo.

En la tabla 3.11 se resumen los valores medios de las métricas calculadas para cada arroyo en el segundo periodo. La riqueza taxonómica y la densidad fueron significativamente mayores en los sitios de referencia (35 taxones y $845 \mathrm{ind} / \mathrm{m}^{2}$ ) en comparación con los sitios hortícolas (22 taxones y 274 ind $/ \mathrm{m}^{2}, p=<0,001$ ). Sin Nombre fue el arroyo con mayor riqueza taxonómica (40 taxones registrados) y Destino fue el arroyo con mayor densidad $\left(1081 \mathrm{ind} / \mathrm{m}^{2}\right)$, mientras que el arroyo Martín fue el de menor riqueza (14 taxones) y El Sauce presentó la menor densidad (147 ind $/ \mathrm{m}^{2}$ ). La diversidad $\left(H^{\prime}\right)$ fue mayor en los sitios de referencia que en los sitios hortícolas $(p=0,046)$, con un valor medio de 2,2 en arroyos de referencia y 1,8 en sitios con horticultura, siendo el más diverso Sin Nombre y el menos diverso el Gato.

Tabla 3.11. Métricas promedio por arroyo.

\begin{tabular}{cccc|cccc} 
& \multicolumn{3}{c|}{ HORTíCOLAS } & \multicolumn{4}{c}{ REFERENCIA } \\
\hline & Martín & Gato & El Sauce & Sin Nombre & Buñirigo & Morales & Destino \\
\hline Riqueza & 14 & 24 & 26 & 40 & 31 & 32 & 35 \\
Densidad (ind $\left./ \mathrm{m}^{2}\right)$ & 265 & 408 & 147 & 999 & 490 & 800 & 1081 \\
Diversidad $\left(H^{\prime}\right)$ & 1,98 & 1,06 & 2,12 & 2,43 & 1,88 & 2,36 & 2,29 \\
\hline
\end{tabular}

El análisis cluster mostró tres agrupamientos de los sitios (fig. 3.7). El grupo A conformado por todas las muestras de sitios de referencia y los grupos B y C conformados por arroyos hortícolas. El grupo B agrupa las muestras de los sitios El Gato y El Sauce que a su vez se agrupan por arroyo, y el grupo C agrupó las muestras del Arroyo Martín y un muestreo de El Sauce. Internamente en el grupo A se agrupan todos los muestreos de Buñirigo mientras que los restantes arroyos de referencia se agrupan por fecha de muestreo. 


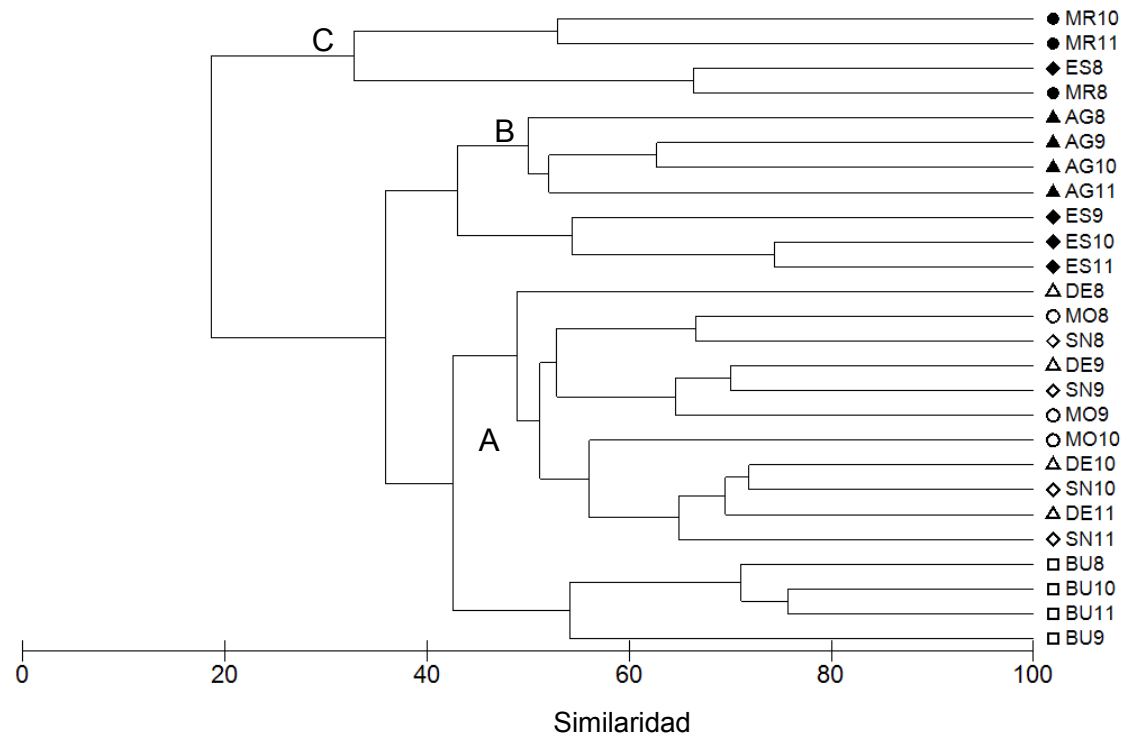

Fig 3.7. CLUSTER de los sitios muestreados en el segundo periodo en base a matriz de similitud con Bray Curtis. Símbolos negros: sitios hortícolas. Símbolos blancos: sitios de referencia. MR. Martín , ES: El Sauce, AG: del Gato, DE: Destino, MO: Morales, SN: Sin

Nombre, BU: Buñirigo. 8: Nov-2016, 9: Dic-2016, 10: Ene-2017, 11: Mar-2017.

La composición del ensamble en arroyos hortícolas y de referencia fue significativamente diferente (ANOSIM: $R$ Global =0,67 y $p=<0,001$ ). El análisis SIMPER (tabla 3.12) mostró una disimilaridad promedio de $73 \%$ entre ambos grupos. Los colémbolos (Entomobryioidea) fueron el grupo que más contribuyó a la diferencia entre la composición del ensamble debido a su mayor densidad en arroyos hortícolas, seguido Hyalella y $S$. vetulus por sus mayores densidades en arroyos menos impactados, y Baetidae por su ausencia en sitios hortícolas. 
Tabla 3.12. Análisis SIMPER mostrando abundancias medias (transformadas por logaritmo) de los taxones y su contribución al $50 \%$ de la disimilaridad.

\begin{tabular}{ccccc}
\hline Taxones & \multicolumn{2}{c}{ Abundancia promedio } & Contribución & Contribución \\
& Hortícolas & Referencia & $(\%)$ & Acum. (\%) \\
\hline Entomobryoidea & 4,3 & 1,1 & 5,1 & 5,1 \\
Hyalella & 1,7 & 4,3 & 4,9 & 10,0 \\
Baetidae & 0 & 2,9 & 4,4 & 14,4 \\
S. vetulus & 1,1 & 3,6 & 4,2 & 18,6 \\
Drepanotrema & 0,7 & 3,2 & 3,9 & 22,6 \\
S. daphnoides & 0,3 & 2,6 & 3,5 & 26,0 \\
Coenagrionidae & 1,7 & 2,9 & 3,1 & 29,1 \\
Chironomidae & 0,7 & 2,7 & 3,1 & 32,2 \\
Liodessus & 1,0 & 2,5 & 3,0 & 35,2 \\
Ciclopoidea & 1,2 & 2,8 & 3,0 & 38,2 \\
Tropisternus & 0 & 2,0 & 2,8 & 41,0 \\
Oligochaeta & 1,0 & 2,1 & 2,7 & 43,7 \\
Diaphanosoma & 0,1 & 1,7 & 2,7 & 46,4 \\
Caenis & 0 & 1,4 & 2,5 & 48,9 \\
\hline
\end{tabular}

\section{Correlaciones}

Las concentraciones de plaguicidas mostraron una correlación positiva con las concentraciones de nutrientes (tabla 3.13): los valores de nitrito estuvieron positivamente relacionados con la sumatoria de los distintos isómeros de endosulfán $(R=0,52$ y $p=0,01)$, las concentraciones de nitrato con la sumatoria de DDTs $(R=0,47$ y $p=0,025)$ y $P R S$ al endosulfán total y DDTs $(R=0,47$ y $p=0,024 ; R=0,47$ y $p=0,025$; respectivamente). Las concentraciones de PRS y nitrato mostraron una correlación positiva con el porcentaje ocupado por horticultura en la cuenca $(R=0,67$ y $p=0,0002$; $R=0,58$ y $p=0,002$, respectivamente).

Tabla 3.13. Correlaciones entre concentraciones de nutrientes, plaguicidas y $\%$ de horticultura en la cuenca. En negritas se muestran los valores significativos $(p<0,05)$.

\begin{tabular}{cccc}
\hline & $\sum$ Endosulfanes & $\sum$ DDTs & \% Ocupado por Horticultura \\
\hline Nitritos & $\mathbf{0 , 5 2}$ & 0,24 & 0,32 \\
Nitratos & 0,36 & $\mathbf{0 , 4 7}$ & $\mathbf{0 , 5 8}$ \\
PRS & $\mathbf{0 , 4 7}$ & $\mathbf{0 , 4 7}$ & $\mathbf{0 , 6 7}$ \\
\hline
\end{tabular}


La densidad se correlacionó positivamente con la cobertura de la vegetación $(R=0,47, p=0,014)$. La riqueza y la densidad se relacionaron inversamente con PRS $(R=-0,67$ y $p=<0,001 ; R=-0,4$ y $p=0,04$ respectivamente) y al nitrato $(R=-0,43$ y $p=0,028 ; R=0,4$ y $p=0,049$ respectivamente), mientras que la diversidad $\left(H^{\prime}\right)$ se correlacionó de manera negativa con la concentración de PRS $(R=-0,589$ y $p=0,001)$. La riqueza y la diversidad $\left(\mathrm{H}^{\prime}\right)$ se correlacionaron negativamente a las concentraciones de clorpirifos $(R=-0,424 ; p=0,044$ ambos) y a la sumatoria de plaguicidas totales $(R=-$ 0,492 y $p=0,017$ ambos), además la diversidad presentó una tendencia negativa en relación a la sumatoria de los isómeros de endosulfán ( $R=-0,39$ y $p=0,06)$. Tanto la riqueza como la densidad se correlacionaron negativamente con el porcentaje de la cuenca ocupado por horticultura $(R=-0,66$ y $p=0,0003 ; R=-0,67$ y $p=0,0002$, respectivamente).

Se analizó la relación de los taxones más representativos con el porcentaje de horticultura en la cuenca. S. vetulus, Hyalella y Baetidae se correlacionaron negativamente con el porcentaje de horticultura, sólo Entomobryoidea se correlacionó positivamente. Los valores de significancia y del coeficiente de correlación se muestran en la siguiente tabla (3.14).

Tabla 3.14. Correlaciones entre taxones y porcentaje ocupado por horticultura en la cuenca. En negritas se muestran los valores significativos $(p<0,05)$.

\begin{tabular}{llc}
\hline \multicolumn{1}{c}{ Taxones } & $\mathrm{R}$ & $\mathrm{p}$ \\
\hline S. vetulus & $\mathbf{- 0 , 4 5 8}$ & $\mathbf{0 , 0 2}$ \\
Hyalella $s p$. & $\mathbf{- 0 , 4 8 9}$ & $\mathbf{0 , 0 1}$ \\
Baetidae & $\mathbf{- 0 , 4 5 4}$ & $\mathbf{0 , 0 2}$ \\
Caenis & $-0,368$ & 0,06 \\
Entomobryoidea & $\mathbf{0 , 4 7}$ & $\mathbf{0 , 0 1}$ \\
Dugesiidae & 0,337 & 0,09 \\
C. incisa & 0,2 & 0,33 \\
Hirudinea & 0,364 & 0,07 \\
\hline
\end{tabular}

\section{Análisis multivariados}

El análisis de redundancia indicó que el fósforo reactivo soluble, la clorofila, el $\mathrm{pH}$ y la cobertura de la vegetación explicaron el $52 \%$ de la variabilidad total en el ensamble de invertebrados. La significancia global para el primer eje canónico y para la suma de todos los ejes fue significativo $(F=7,4 ; p=0,002$ y $F=2,35$; $p=0,002$, respectivamente). Se realizó un análisis parcial utilizando la vegetación 
como covariable. En este análisis, las variables que resultaron significativas fueron fósforo reactivo soluble, el $\mathrm{pH}$, la clorofila y el carbono orgánico. Este RDA explicó el $42 \%$ de la variación remanente. El primer y el segundo eje explicaron el $86 \%$ de la relación entre los taxones y el ambiente. El primer eje se correlacionó con PRS $(-0,65)$, $\mathrm{pH}(0,57)$, clorofila (Clor) $(0,34)$ y el porcentaje de carbono orgánico en el sedimento (Corg\%) $(-0,18)$. El segundo eje estuvo correlacionado con clorofila (Clor) $(0,63), \mathrm{C}$ org $\%(0,48)$ y PRS $(-0,32)$.

En diagrama de sitios de muestreo (Fig. 3.8, izquierda), las muestras de arroyos hortícolas se localizaron en el 3er cuadrante, relacionadas a altas concentraciones de PRS y el Carbono orgánico (\%), reflejando el uso del suelo hortícola. Las muestras de arroyos sin horticultura se ubicaron de manera opuesta a las concentraciones de PRS y carbono orgánico (\%). Este análisis mostró una correspondencia de abundancias con ciertos taxones de invertebrados a los gradientes (Fig 3.8, derecha). Entomobryoidea mostró una relación positiva con el PRS y C. incisa se relacionó con el carbono orgánico. Contrariamente, Caenis, Hyalella, Diaphanosoma, Ephydridae y Baetidae se relacionaron negativamente con el PRS y el carbono orgánico. Las especies de Simocephalus (S. vetulus y S. daphnoides), los coleópteros Paracymus, Liodessus, Tropisternus y Chironomidae estuvieron positivamente relacionados al $\mathrm{pH}$, mientras que Oligochaeta, Belostoma, Coenagrionidae, Drepanotrema, Ciclopoidea y Biomphalaria y Uncancylus se relacionaron además con la clorofila.
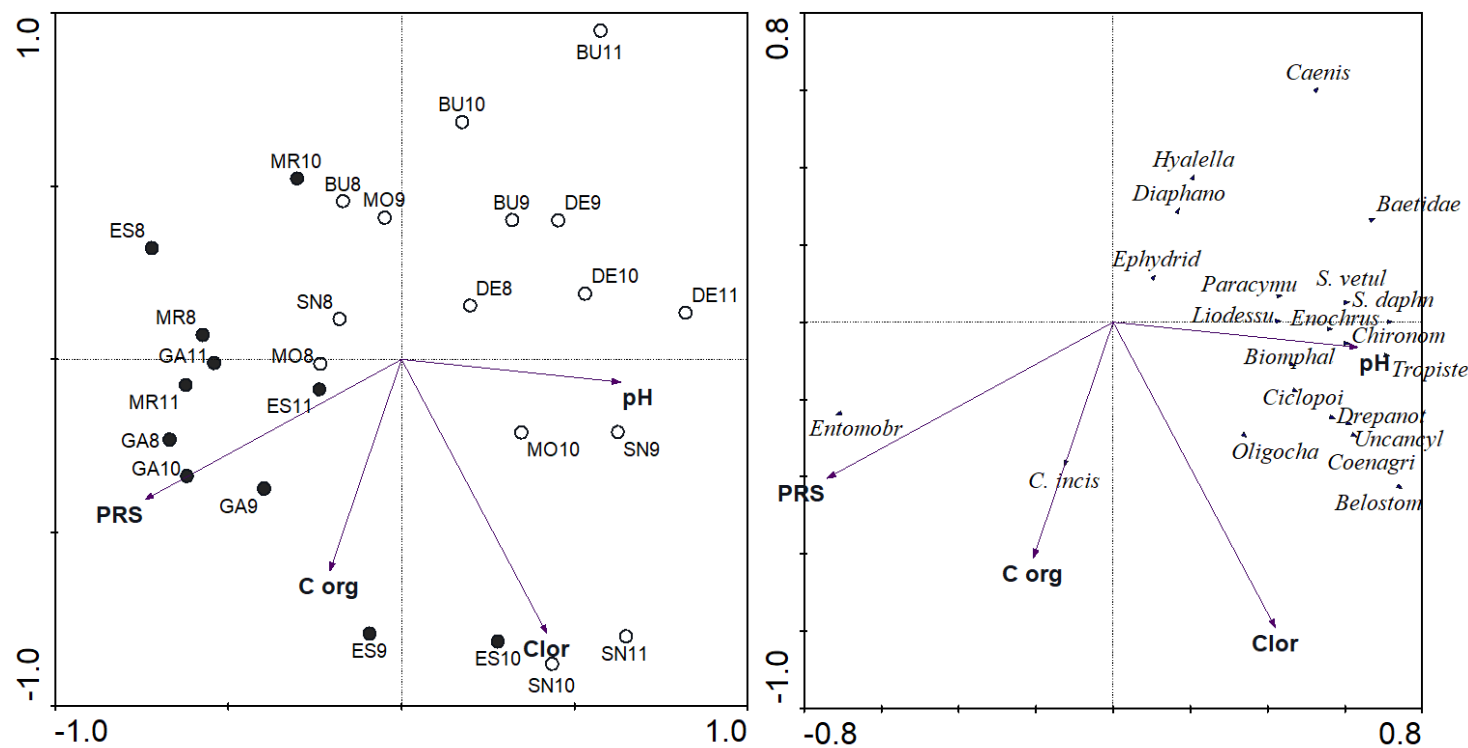

Fig. 3.8. Análisis de Redundancia Parcial. Gráficos de datos ambientales y especies (izquierda) y sitios (derecha). Círculos blancos: sitios sin horticultura; círculos negros: sitios hortícolas. MR: Martín, GA: Gato, ES: Sauce, SN: Sin Nombre, BU: Buñirigo, MO: Morales, DE: Destino. 8: Nov-2016, 9: Dic-2016, 10: Ene-2017, 11: Mar-2017. Se muestran solo las variables ambientales que explicaron significativamente el ensamble de invertebrados. 


\subsection{Comparación entre ambos periodos}

El primer periodo abarcó una mayor amplitud temporal (de octubre a mayo) y un mayor número de muestras, mientras que en el segundo periodo los muestreos se realizaron en primavera y verano (de noviembre a marzo), siendo el número de muestras aproximadamente la mitad que el primer periodo. El arroyo San Juan permaneció seco durante el segundo periodo de muestreo, resultando en un menor número de muestras de arroyos hortícolas.

En el primer periodo no se encontraron diferencias significativas entre las variables ambientales medidas, no obstante en el análisis multivariado se observa que los sitios de referencia se ordenaron por un gradiente de conductividad. En el segundo periodo, los arroyos de referencia presentaron mayores temperaturas y valores de $\mathrm{pH}$ más alcalinos que los arroyos hortícolas. También presentaron mayor concentración de sólidos suspendidos y una tendencia a altos valores de conductividad. Las mayores temperaturas del agua pueden deberse a la escasez de sombra que presentan estos arroyos y que el periodo de muestreo estuvo concentrado en los meses de mayor temperatura. La clorofila estuvo positivamente relacionada a las concentraciones de fósforo en el primer periodo, contrariamente en el segundo periodo dichas variables no presentaron correlación.

En ambos periodos, las concentraciones de nutrientes en los arroyos hortícolas fueron significativamente mayores que en los arroyos de referencia, y estuvieron positivamente correlacionadas con el porcentaje de horticultura en la cuenca. En ambos periodos en el análisis multivariado los sitios hortícolas se ordenaron en relación a las concentraciones de PRS, además en el primer periodo en base al amonio y en el segundo al carbono orgánico del sedimento. En el primer periodo los arroyos de referencia se ordenaron en función de las bajas concentraciones de nutrientes y altas conductividades, mientras que en el segundo año se ordenaron en función del pH y la clorofila.

Con respecto a los plaguicidas, en general las determinaciones fueron escasas, con algunos registros altos aislados. En el primer periodo los arroyos hortícolas presentaron mayor frecuencia de detección y en el segundo periodo las concentraciones de endosulfan fueron significativamente mayores en arroyos hortícolas. Además, en el segundo periodo fue posible establecer correlaciones positivas entre las concentraciones de insecticidas y las concentraciones de nutrientes y negativas con las métricas de los ensambles. 
Los ensambles de invertebrados asociados a vegetación presentaron características similares en ambos periodos con respecto al uso del suelo. En ambos periodos se determinaron mayor riqueza y densidad en arroyos de referencia que en los arroyos hortícolas. La riqueza estuvo negativamente correlacionada con el porcentaje de horticultura en la cuenca en ambos periodos y también la diversidad en el segundo periodo.

Los taxones S. vetulus, Hyalella y Baetidae caracterizaron los sitios de referencia mientras que Collembola fue característico de los sitios con horticultura. Trichoptera (Cyrnellus e Hidroptylidae), Decapoda (Palaeomonetes, Aeglidae y Trichodactylidae) y el efemeróptero Caenis aparecieron sólo en sitios de bajo impacto, mientras que los arroyos hortícolas presentaron mayores densidades de Hirudinea y Dugessidae en el primer periodo y $C$. incisa en el segundo. El análisis de cluster mostró tres agrupamientos en ambos periodos, un grupo para arroyos de referencia, y dos grupos para arroyos hortícolas. El análisis de redundancia presentó una tendencia general de ordenamiento con el PRS como variable más representativa, ordenando los sitios hortícolas hacia mayores valores y los sitios de referencia hacia menores valores, indicando el uso del suelo en la cuenca. Entomobryioidea estuvo relacionado al carbono orgánico en el primero periodo y con el PRS en el segundo, Hirudinea y Dugesiidae con el PRS y C. incisa con el carbono orgánico, mientras que $S$. vetulus, Hyalella, Baetidae, Caenis, S. daphnoides, Tropisternus, Biomphalaria y Drepanotrema estuvieron relacionadas a bajas concentraciones de PRS. Contrariamente, Paracymus y Enochrus se relacionaron a altas concentraciones de PRS en el primer año y a bajas concentraciones de PRS durante el segundo.

\subsection{Influencia de la vegetación y el uso del suelo sobre los ensambles}

La vegetación fue importante para la estructuración de los ensambles ya que la densidad de invertebrados estuvo correlacionada positivamente con la cobertura de la vegetación en ambos periodos y a la riqueza en el primero, como así también, el porcentaje de cobertura de la vegetación fue una variable que explicó significativamente el modelo de ordenamiento en los análisis multivariados (pág. 24 primer periodo y pág. 33 segundo periodo).

La vegetación de los arroyos estudiados se clasificó siguiendo a Walker et al. (2013) con modificaciones (tabla 3.15). 
Tabla 3.15. Tipos de vegetación determinados en los arroyos

\begin{tabular}{|c|c|c|}
\hline Tipo & Vegetación & Arroyos \\
\hline $\begin{array}{c}\text { Carpeta } \\
\text { (A) }\end{array}$ & $\begin{array}{l}\text { Plantas flotantes formando carpeta. Lemna, Azolla, } \\
\text { Spirodela }(+75 \%) \text { acompañada por otras especies } \\
\text { (Sagittaria, Ludwigia) }\end{array}$ & San Juan, Morales \\
\hline $\begin{array}{l}\text { Simple } \\
\text { (B) }\end{array}$ & $\begin{array}{l}\text { Plantas emergentes (75\%) con poco desarrollo de } \\
\text { vegetación sumergida (25\%), representado por } \\
\text { Gymnocoronis, Polygonum, Hydrocleys, Ceratophyllum }\end{array}$ & $\begin{array}{c}\text { Martín , Sauce, } \\
\text { Buñirigo }\end{array}$ \\
\hline $\begin{array}{l}\text { Compleja } \\
\qquad \text { (C) }\end{array}$ & $\begin{array}{c}\text { Diferentes tipos de vegetación, gran desarrollo de } \\
\text { vegetación sumergida (50\%): Ludwigia, Elodea, } \\
\text { Myriophyllum, Ceratophyllum }\end{array}$ & $\begin{array}{c}\text { Gato, Sin Nombre, } \\
\text { Destino }\end{array}$ \\
\hline
\end{tabular}

Los arroyos San Juan y Morales presentaron vegetación de tipo carpeta de plantas flotantes de pequeño tamaño y escaso desarrollo radicular, pero una alta cobertura. Este tipo de vegetación presentó en su mayoría bajos valores de oxígeno disuelto y clorofila, a excepción de valores altos de oxígeno (8,06-10 mg/L) en Octubre 2015 y 355 g/L clorofila en San Juan en Octubre 2014. Los arroyos Martín, Sauce y Buñirigo presentaron vegetación simple con Gymnocoronis como dominante, con abundante desarrollo de raíces y asociada a vegetación sumergida en escasa proporción, con un rango de cobertura variable; este tipo vegetacional estuvo acompañado muchas veces de algas filamentosas, altos valores de oxígeno y escasa clorofila (a excepción de dos valores extremos, uno en El Sauce y otro en Martín ). Los arroyos Gato, Sin Nombre y Destino presentaron una vegetación compleja, conformada por especies arraigadas y sumergidas como Ludwigia y como Myriophyllum, con gran desarrollo de hojas sumergidas y de sistema radicular, con cobertura en general alta; en general valores altos de oxígeno, presencia de algas filamentosas y alta concentración de materia orgánica, debido a la gran cantidad de vegetación.

Se comparó entonces la densidad media $\left(\mathrm{ind} / \mathrm{m}^{2}\right)$ de los órdenes de invertebrados según el uso del suelo y según el tipo vegetacional. En la figura 3.9 se observa la comparación entre los arroyos Morales y San Juan, ambos con tipo de vegetación carpeta. Se puede observar una densidad media de entre 300 y 600 ind/m2, con una dominancia de anfípodos en Morales y de Dípteros y Platelmintos en San Juan. Se observa también la presencia de ephemeroptera en Morales, ausente en San Juan. 
En la figura 3.10 se observa la comparación entre los arroyos con vegetación simple. Se observa una menor densidad media en comparación con el tipo de vegetación carpeta, aproximadamente $200 \mathrm{ind} / \mathrm{m}^{2}$, en Buñirigo una mayor densidad de cladóceros y anfípodos y en El Sauce y Martín una mayor densidad de colémbolos.

En el gráfico de comparación entre arroyos con vegetación compleja (fig 3.11) se observa una mayor densidad de individuos que en los ensambles de vegetación simple, pero menor densidad que los ensambles asociados a carpeta vegetal. Se observa también una mayor cantidad de grupos bien representados, aunque con una dominancia de cladóceros y anfípodos en los arroyos Sin nombre y Destino y por colémbolos, copépodos y anfípodos en el arroyo Gato.

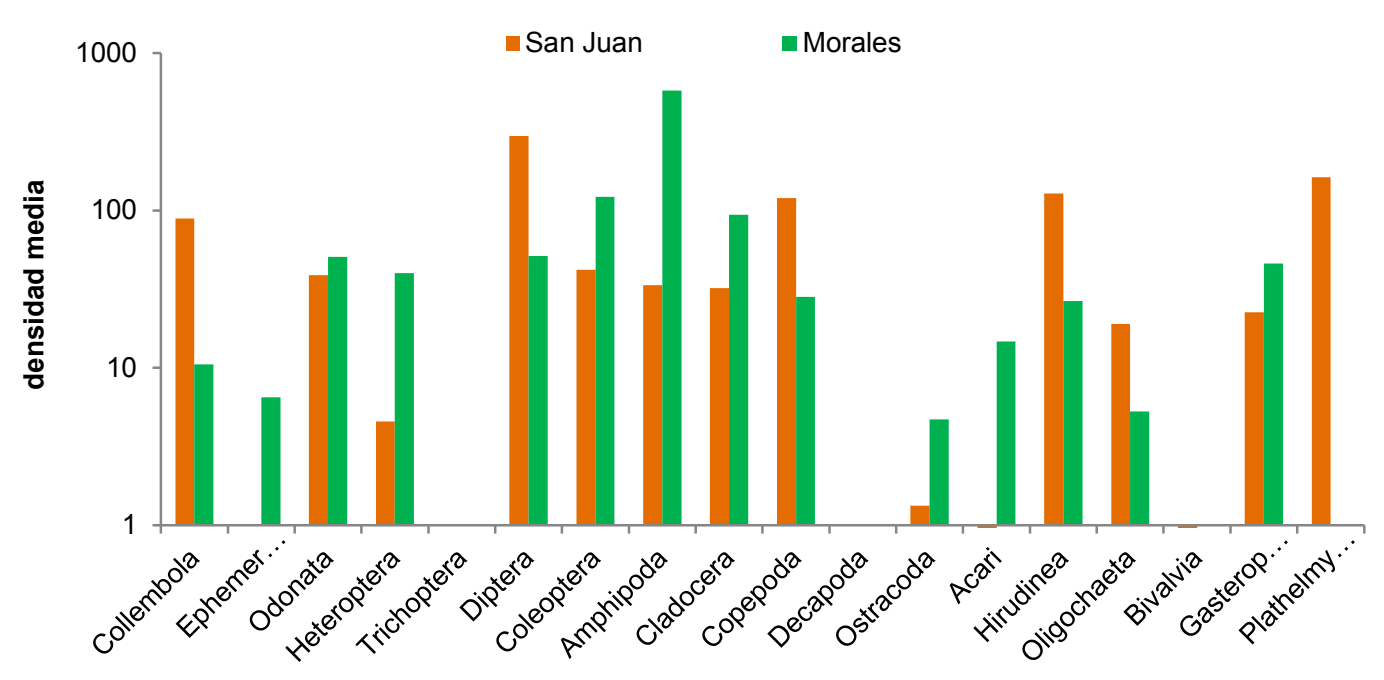

Figura 3.9. Densidad media (logarítmica) de arroyos con vegetación carpeta

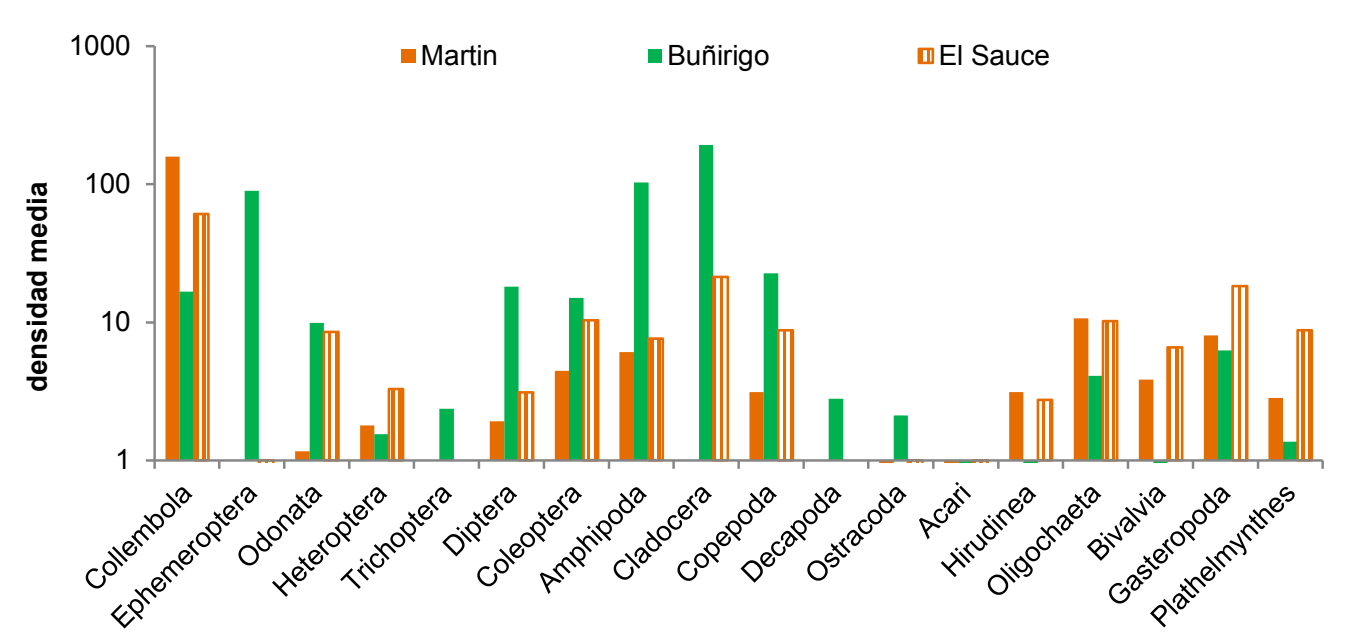

Figura 3.10. . Densidad media (logarítmica) de arroyos con vegetación simple 


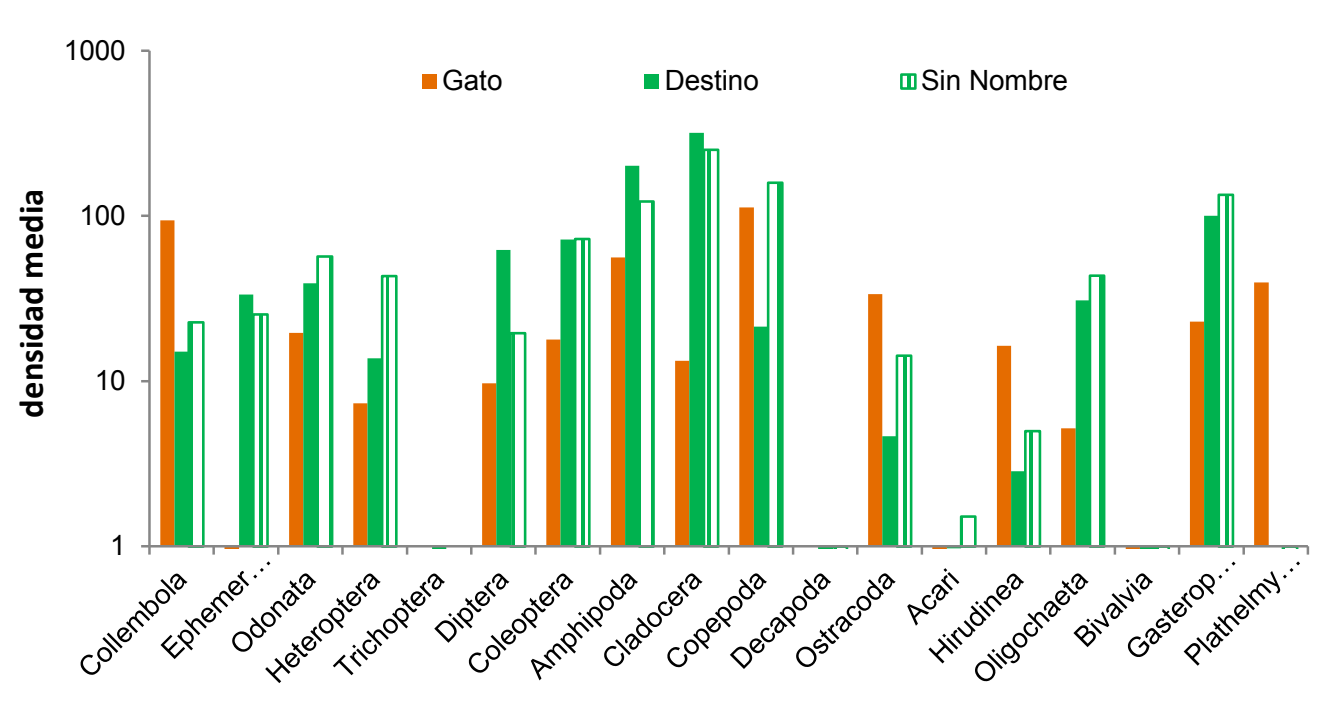

Figura 3.11. . Densidad media (logarítmica) de arroyos con vegetación compleja

Se compararon las métricas riqueza, densidad (ind $\left./ \mathrm{m}^{2}\right)$ y diversidad $\left(\mathrm{H}^{\prime}\right)$ de los arroyos que presentaron igual vegetación y distinto uso del suelo. Los resultados se muestran en la tabla 3.16.

Tabla 3.16. Comparación de las métricas entre los arroyos con el mismo tipo de vegetación. Se muestran los valores de significancia.

\begin{tabular}{ccccc}
\hline & & Riqueza & Densidad & Diversidad \\
\hline CARPETA & & & & \\
& San Juan vs. Morales & $\mathbf{0 , 0 0 2}$ & 0,83 & 0,71 \\
\hline SIMPLE & & & & \\
& El Sauce vs. Buñirigo & $\mathbf{0 , 0 4 3}$ & $\mathbf{0 , 0 1 1}$ & 0,16 \\
& Martín vs. Buñirigo & $<0,001$ & 0,056 & 0,13 \\
\hline COMPLEJA & & & & \\
& Gato vs. Sin Nombre & $\mathbf{0 , 0 0 1}$ & $\mathbf{0 , 0 0 2}$ & $\mathbf{0 , 0 4}$ \\
& Gato vs. Destino & 0,055 & 0,2 & 0,26 \\
\hline
\end{tabular}

En general los arroyos fueron diferentes en cuanto al número de especies aún con el mismo tipo de vegetación, a excepción de los arroyos Gato y Destino que no presentaron diferencias entre sí en ninguna métrica. La densidad fue diferente entre el Sauce y Buñirigo ambos con vegetación simple, y entre el Gato y Sin Nombre que presentaron además diferencias significativas en la diversidad. 
Se realizó un ANOSIM de dos vías con los factores "vegetación" y "uso del suelo" para evaluar si existían diferencias en el ensamble de los tipos de vegetación y si, a su vez, fueron diferentes los usos del suelo dentro de cada tipo de vegetación. Se determinó que los tipos de vegetación presentaron ensambles diferentes (R Global= $0,22 ; p=0,002)$. Se realizó un análisis de a pares y se determinó que el ensamble de invertebrados de vegetación simple fue diferente de la carpeta $(R=0,36$ y $p=0,002)$ y de la vegetación compleja $(R=0,24$ y $p=0,003)$, pero el ensamble de invertebrados de vegetación carpeta y compleja no difirieron entre sí $(p=0,35)$. Aun así, la diferencia entre usos del suelo fue mayor que la diferencia entre los tipos de vegetación $(R$ Globlal=0,545 y $p=0,001$ ), por lo tanto el uso del suelo tiene mayor influencia que el tipo de vegetación en la composición de los ensambles.

\section{Discusión}

\section{Variables ambientales}

En general los arroyos estudiados presentaron características similares en cuanto a las variables ambientales. No obstante, todos los sitios asociados a horticultura presentaron mayores concentraciones de nutrientes que los sitios de referencia. Estas mayores concentraciones de nutrientes sugieren el aporte de los fertilizantes aplicados en los cultivos. Repetidamente se informa en la bibliografía elevadas concentraciones de nutrientes en sitios adyacentes a cultivos agrícolas, reflejando el efecto del uso de suelo en la cuenca en la química del agua. Arbuckle y Downing (2001) estudiaron la relación N:P en 113 lagos de lowa y la proporción de uso del suelo agrícola y pasturas dentro de la cuenca. Observaron que el cociente NT/PT se relacionó positivamente con la agricultura y negativamente con las pasturas. Figueiredo et al. (2010) mostraron que el cambio de uso del suelo de pasturas a agricultura incrementa las concentraciones de nitratos en arroyos donde los cultivos bordeaban los márgenes. Mugni et al. (2013b) determinaron mayores concentraciones en un arroyo rodeado por un cultivo fertilizado que el mismo arroyo cuando el cultivo no fue fertilizado. Rimoldi et al. (2018) estudiaron la cuenca del arroyo del Gato y determinaron en los sitios de muestreo de la cuenca baja (cercanos al sitio de muestreo del presente trabajo) elevadas concentraciones de nutrientes, $15-20 \mathrm{mg} \mathrm{N}$ $\mathrm{NO}_{3}{ }^{-} / \mathrm{L}$ de nitratos, 1,3 de fósforo y $0,4-14 \mathrm{mg} / \mathrm{L}$ de amonio, y estos sitios presentaron la peor calidad del agua. Los trabajos anteriormente citados excepto Rimoldi et al. (2018) corresponden a estudios sobre el uso del suelo agrícola, los estudios sobre 
concentraciones de nutrientes en arroyos hortícolas son escasos. Los resultados del presente trabajo de tesis indican que el efecto de la horticultura sería semejante al de la agricultura, en ambos casos hay una transferencia de nutrientes a los arroyos debido a la fertilización de los cultivos en la cuenca.

La clorofila estuvo positivamente relacionada a las concentraciones de PRS en el primer periodo, se podría prever un incremento de clorofila debido al incremento de nutrientes (Wetzel 1981), sin embargo no se determinaron mayores concentraciones de clorofila en los arroyos hortícolas. La presencia de macrófitas y la turbidez del agua posiblemente moderen el efecto del aumento de nutrientes sobre la abundancia de fitoplancton.

Los arroyos de referencia se ordenaron en función de la conductividad en el primer periodo. En estos arroyos el nivel freático está cerca de la superficie. Cellone et al. (2018) estudiaron el agua subterránea de Punta Indio, una zona cercana a los sitios de muestreo de la Reserva y determinaron valores de conductividad de entre 2000 y $3000 \mu \mathrm{S} / \mathrm{cm}$. El presente trabajo se realizó en los meses cálidos, donde el caudal de los arroyos disminuye debido a una mayor evapotranspiración (Sala et al. 1973). Los mayores niveles de conductividad podrían deberse tanto al aporte de las aguas subterráneas como a la evapotranspiración. Por el contrario, en la zona hortícola, la mayor necesidad de agua para riego debido a la impermeabilización del suelo por los invernaderos, sumado a la explotación del agua subterránea para el abastecimiento de la ciudad de La Plata y otras localidades cercanas produjo un cono de depresión de los niveles freáticos y del acuífero Puelche (Laurencena et al. 2008), lo que genera que en las nacientes de los arroyos de la zona periurbana el nivel freático esté a más de $15 \mathrm{~m}$ como fue observado por Varela et al. (2002) en el Arroyo del Gato.

En el segundo periodo los arroyos de referencia presentaron mayores registros de temperatura, $\mathrm{pH}$ y sólidos suspendidos, y en el análisis de redundancia las muestras de los sitios de referencia se ordenaron en función del incremento de $\mathrm{pH}$ y clorofila. Zhang et al. (2018) estudiaron las comunidades de invertebrados de más de 350 sitios en la cuenca del Rio Hun-Tai en China, analizando el porcentaje de pasturas, áreas agrícolas, áreas residenciales y bosque en cada sitio. Agruparon los sitios en áreas menos impactadas, áreas moderadamente impactadas y áreas altamente impactadas; los sitios más impactados, relacionados con menor densidad y diversidad de familias de invertebrados, presentaron mayores valores de fósforo total y menores de $\mathrm{pH}$, mientras que los sitios de impacto intermedio presentaron menores valores de fósforo total y mayores de $\mathrm{pH}$, similar a los resultados del presente trabajo. 
Molina et al (2017) estudiaron la influencia de diferentes usos del suelo: bosque nativo, bosque implantado, vegetación arbustiva y herbácea, ganadería (intensiva y extensiva), cultivos intensivos y usos mixtos (cultivo de café y cítricos, área urbana, autopistas, instalaciones rurales) en arroyos de Brasil, a diferentes escalas: local, red de drenaje (uso del suelo en los $30 \mathrm{~m}$ de zona buffer del arroyo) y cuenca. Determinaron un agrupamiento de arroyos con alta conductividad, sólidos disueltos, $\mathrm{pH}$ y temperatura del agua asociados a áreas con ganadería, presencia de vegetación arbustiva y herbácea en la cuenca (sin sombreado) y a áreas con uso del suelo mixto, y en un diagrama de ordenamiento estos sitios se ordenaron de manera opuesta a los cultivos intensivos (caña de azúcar). Los autores plantean que el ganado remueve el fondo y genera un incremento en sólidos en suspensión, incrementando la turbidez del agua. En el presente trabajo, en los arroyos de referencia se realiza ganadería extensiva, incluso en la Reserva; en estos sitios se observó la presencia de ganado en varias oportunidades, también de pisadas y materia fecal dentro y en los alrededores del cauce, no así en los arroyos hortícolas. Las mayores concentraciones de sólidos en suspensión podrían deberse a la resuspensión de material particulado por efecto del ganado en estos arroyos. Asimismo, el pastoreo y pisoteo seguramente disminuyen la cobertura y generan mayor radiación (por escasez de sombra), incrementando la temperatura del agua y la evapotranspiración, lo que podría influir en la conductividad junto al aporte de las aguas subterráneas.

\section{Plaguicidas}

Los presentes resultados de la frecuencia de detección de insecticidas y las mayores concentraciones determinadas en arroyos con horticultura son consistentes con la literatura. Kreuger et al. (2008) estudiaron arroyos y ríos hortícolas en Suecia, detectando regularmente fungicidas e insecticidas y varios compuestos, principalmente los piretroides, excedieron los niveles guía de calidad de aguas de la legislación Sueca. Kammerbauer y Moncada (1998) estudiaron varios sistemas de agua dulce (ríos, agua de pozo y lagunas) en Honduras con uso de suelo agrícola y hortícola. Las muestras de agua de sitios hortícolas presentaron mayor número de plaguicidas detectados y una mayor frecuencia de detección que los sitios agrícolas. A su vez, en los sitios hortícolas se determinaron las mayores concentraciones de p,p'-DDT, heptacloro, endosulfán y clorpirifos. Mac Laughlin et al. (2017) estudiaron la concentración de plaguicidas en los sedimentos del fondo del Arroyo Carnaval, localizado dentro del área de estudio del presente trabajo. Encontraron altas y frecuentes concentraciones de clorpirifos, piretroides y AMPA (producto de 
degradación de glifosato). Se determinaron concentraciones elevadas de clorpirifos $(2258 \mathrm{ng} / \mathrm{g})$ y lambdacialotrina $(649 \mathrm{ng} / \mathrm{g})$. El patrón registrado en el presente estudio es consistente con las concentraciones medidas de clorpirifos y endosulfan en aire, en el mismo área de estudio (Colombo et al. 2015). Las concentraciones máximas en aire fueron medidas en Olmos, en un sitio ubicado entre los arroyos El Sauce y El Gato; mientras que los valores mínimos fueron determinados en una cuenca ganadera en Magdalena, cercano a los sitios de referencia del presente trabajo. Las detecciones ocasionales y en bajas concentraciones de los arroyos de referencia son consistentes con el transporte atmosférico de largo alcance de los sitios de aplicación (Weber et al., 2010).

Las concentraciones de plaguicidas medidas en los arroyos hortícolas del presente trabajo sugieren la contribución de los cultivos adyacentes. Las altas concentraciones de nutrientes y las mayores concentraciones y frecuencias de detección de plaguicidas en los arroyos hortícolas sumado a las correlaciones positivas entre las concentraciones de nutrientes, el porcentaje de horticultura y las concentraciones de plaguicidas, indicarían la incorporación simultánea de nutrientes y plaguicidas a los arroyos en coincidencia con eventos de escorrentía posteriores a las aplicaciones en los cultivos. Esto estuvo representado tanto por los gradientes de PRS obtenidos en los análisis multivariados en ambos periodos, indicando el uso del suelo, como por las correlaciones de las concentraciones de nutrientes y las concentraciones de plaguicidas en el segundo periodo. Mangiafico et al. (2009) estudiaron la escorrentía producida por lluvias en diferentes cultivos (viveros y cultivos de cítricos y paltas) en California, determinando las concentraciones de nitritos, nitratos y plaguicidas en la escorrentía superficial. Las concentraciones medias de nitrito-nitrato fueron aproximadamente $5(0,07$ a 31) $\mathrm{mg}$ N-NO3/L y para PRS $1,5(0,01-8,1) \mathrm{mg} / \mathrm{L}$, estos últimos excediendo los valores de referencia del agua de USEPA. Los valores de piretroides, organofosforados y organoclorados fueron en general menores a la CL50 de Daphnia magna, excepto clorpirifos y organoclorados (DDT, DDE y DDD). Egler et al. (2012) determinaron mayores valores de nutrientes y concentraciones de plaguicidas en arroyos con agricultura que en sitios con bosques o pasturas. Asimismo, Marrochi (2018) determinó correlaciones positivas entre las concentraciones de PRS, clorpirifos, plaguicidas totales y unidades tóxicas en arroyos con agricultura en Arrecifes, al norte de la provincia de Buenos Aires. Estos trabajos confirman que los nutrientes y plaguicidas que se aplican en los cultivos ingresan a los arroyos con la escorrentía superficial. Como se mencionó anteriormente, la mayoría de la bibliografía remite a estudios en arroyos agrícolas, la información disponible sobre 
cuencas hortícolas es escasa; el presente trabajo indica que el efecto del uso de agroquímicos en la horticultura sería similar a la agricultura.

\section{Ensambles de invertebrados}

Los arroyos hortícolas presentaron menor riqueza y menor densidad de invertebrados que los arroyos de referencia. A su vez, en ambos periodos existió una correlación negativa entre el porcentaje de horticultura en la cuenca y las concentraciones de nutrientes con la riqueza de taxones, y en el segundo periodo una correlación negativa entre la riqueza de taxones y las concentraciones de clorpirifos y plaguicidas totales. El número de especies es un índice natural de la estructura de la comunidad (Magurran y McGill 2011) y es una métrica importante en los estudios de comunidades ya que la presencia o ausencia de un grupo es un indicador del estado de los ecosistemas; este enfoque es la base de muchos índices bióticos de la calidad del agua con invertebrados (Barbour et al. 1999, Figueroa et al. 2003, Rios-Touma et al. 2014). Beketov et al. (2013) estudiaron el efecto de los plaguicidas en la riqueza taxonómica regional de invertebrados en arroyos de Europa y Australia, considerando trabajos que incluyeron determinaciones de la exposición a plaguicidas y medidas de factores ambientales. Compararon grupos de sitios caracterizados por niveles de contaminación por plaguicidas determinados por unidades tóxicas, que es la relación entre valores de plaguicidas determinados y la CL50 de Daphnia magna (ver Tomlin 2000, Liess y van der Ohe 2005), concluyendo que la exposición a los plaguicidas causó la disminución de la riqueza en ambas regiones, tanto a nivel de especies como a nivel de familia. Los plaguicidas causan mortalidad en algunas especies, provocando la desaparición de algún grupo en los arroyos o la disminución de la densidad de otros, lo que genera la disminución de la riqueza y de la densidad del ensamble de invertebrados.

En el presente estudio, los crustáceos $S$. vetulus y Hyalella sp. y los efemerópteros Baetidae y Caenis sp. fueron los taxones mejor representados en los sitios de referencia, mostrando una fuerte reducción de su abundancia en los arroyos hortícolas y se relacionaron de manera opuesta al gradiente que indicó uso del suelo en los análisis de redundancia (representado por PRS, amonio y carbono orgánico). Los cladóceros de la familia Daphniidae son los más comúnmente utilizados en biomonitoreo debido a su sensibilidad a plaguicidas (Zhou et al., 2008). Simocephalus vetulus es una especie cosmopolita asociada a la vegetación acuática de ambientes eutróficos, muy utilizada como modelo de ensayos de toxicidad (Chen et al., 2004; Schroer et al., 2004; Willis et al., 1995). De manera similar, los anfípodos del género 
Hyalella son utilizados frecuentemente como organismos de monitoreo (Nebeker and Miller, 1989). Mugni et al. (2011) determinaron la toxicidad en agua de escorrentía y del cauce para $H$. curvispina en coincidencia con la primer lluvia con posterioridad a la aplicación en el cultivo adyacente en el $A^{\circ}$ el Sauce, en un sitio cercano al punto de muestreo del presente trabajo. Paracampo et al (2012) determinaron toxicidad en agua de escorrentía para $H$. curvispina en parcelas experimentales de soja con simulaciones de lluvia hasta aproximadamente un mes con posterioridad a cada aplicación de cipermetrina, endosulfán y clorpirifos. Mac Laughlin et al. (2017) estudiaron la toxicidad del sedimento para $H$. curvispina en el Arroyo Carnaval, localizado entre los arroyos San Juan y Martín; registraron mortalidad y una disminución del crecimiento en los organismos expuestos a sedimentos que presentaron las mayores concentraciones de insecticidas. Considerando estos resultados, S. vetulus y Hyalella $s p$. siendo abundantes en los ambientes regionales y sensibles a plaguicidas, pueden ser considerados como indicadores del impacto de plaguicidas en arroyos pampeanos.

Los efemerópteros son considerados en muchos índices y métricas para evaluar calidad ambiental (Barbour et al. 1999), típicamente el índice ETP (Ephemeroptera, Trichoptera y Plecoptera). En general se distribuyen en ambientes bien oxigenados, Baetidae asociado a vegetación y Caenidae a ambientes de corrientes lentas (Domínguez et al. 2009). Caenis es considerado un taxón sensible por Liess y von der Ohe (2005). De manera similar al presente estudio, Suren (1994) determinó una alta abundancia de Baetidae en arroyos con pasturas mientras que estuvo pobremente representado en arroyos agrícolas en Nepal, y Zhang et al. (2018) determinaron menores densidades de Baetidae en los sitios más contaminados y mayores densidades de este grupo en los sitios menos disturbados en China. Trichoptera y Decapoda, ambos considerados sensibles por Liess y von der Ohe (2005) se encontraron sólo en arroyos de referencia en el presente estudio.

Por el contrario, en los arroyos hortícolas Entomobryoidea, Dugessidae e Hirudinea estuvieron bien representados y mostraron bajas densidades en los sitios de referencia durante el primer periodo. En el segundo periodo, los dos taxones que caracterizaron a los sitios hortícolas fueron Entomobryoidea (colémbolos) y C. incisa (ostrácodos). De manera similar, Egler et al. (2012) estudiaron la calidad el agua y los invertebrados bentónicos relacionados con usos de suelo de bosque, pasturas y agricultura intensiva en Brasil, y en arroyos agrícolas determinaron altas densidades de colémbolos junto con bajos valores de riqueza y mayores concentraciones de nutrientes. Wang et al. (2016) determinaron que el agregado tanto de fertilizantes orgánicos (torta de soja) como inorgánicos en plantaciones de álamo produjo un 
aumento de las densidades de colémbolos en suelo, interpretado como resultado de un incremento en la disponibilidad de alimento. Endlweber et al. (2006) estudiaron los efectos de la aplicación de clorpirifos y dimetoato sobre la densidad y la estructura de los colémbolos del suelo, determinando una fuerte reducción de los Sminthuroidea luego de las aplicaciones y un incremento en las densidades de Protaphorura (Poduroidea), similar a los resultados de este trabajo de tesis. Los autores plantean que este incremento podría deberse a la recolonización más que a la reproducción fundamentalmente en las especies epígeas (como Poduroidea y Entomobryiodea) ya que los sistemas de estudio eran abiertos.

Si bien estos últimos trabajos fueron realizados en ecosistemas terrestres, se hace referencia a procesos ecosistémicos generales: el agregado de fertilizantes incrementa la producción primaria y en consecuencia la materia orgánica, tanto de sistemas terrestres como acuáticos; la recolonización por parte de algunos grupos debido a la disponibilidad de nichos se da en sistemas abiertos que presenten conectividad. La recolonización en ambientes acuáticos es un proceso a escala regional (Palmer et al. 1996), puede darse por aportes de las nacientes (Meyer et al. 2007, Orlinskiy et al. 2015), de la planicie de inundación o el ambiente terrestre circundante (Robinson et al. 2002). Por consiguiente, en los arroyos podrían confluir ambos procesos, tanto la recolonización como el aprovechamiento de los mayores recursos disponibles, derivando en las mayores densidades de colémbolos observadas en los arroyos hortícolas en el presente trabajo.

Dugessidae e Hirudinea son ambos considerados tolerantes por Liess y von der Ohe (2005), por lo que se esperaría una comparativamente elevada abundancia en sitios hortícolas, consistente con las mayores densidades observadas en el presente trabajo. La especie de ostrácodo C. incisa estuvo correlacionada al porcentaje de carbono orgánico en el sedimento. Cortelezzi et al. (2015) estudiaron el efecto del agregado experimental de nutrientes sobre el ensamble de invertebrados en el arroyo La Choza, un arroyo pampeano de la provincia de Buenos Aires, y registraron que los ostrácodos incrementaron su abundancia después de la fertilización.

Se determinó que la estructura de la vegetación influye en la estructura del ensamble de invertebrados ya que la riqueza taxonómica y la densidad estuvieron positivamente correlacionadas a la cobertura vegetal. Begon et al. (2006) plantean que la riqueza específica de los animales está relacionada a la heterogeneidad espacial producida por la vegetación. En los arroyos pampeanos, las macrófitas son las principales responsables de la heterogeneidad del hábitat tanto espacial como temporal (Giorgi et al. 2005). Varios autores han evaluado el rol de la vegetación en la 
estructuración de las comunidades de invertebrados, y se ha determinado que la complejidad de la vegetación está asociada a una mayor abundancia de invertebrados y una mayor riqueza (Ferreiro et al., 2014; Walker et al., 2013). Los resultados indican que a mayor cobertura de la vegetación y mayor complejidad de la misma, la riqueza y la abundancia de invertebrados será mayor. No obstante, el uso del suelo mostró una mayor incidencia en la composición de los taxones: arroyos con uso del suelo y vegetación similares presentarán un ensamble de invertebrados similar, pero si el uso del suelo es diferente, el ensamble de invertebrados será distinto, aunque el tipo de vegetación sea el mismo. Asimismo, la riqueza fue en general menor en los sitios hortícolas, aún con vegetación similar, lo que sustenta a la riqueza como un buen indicador del uso del suelo en estos ambientes. Los análisis de partición de la varianza permiten evaluar las variables de manera diferencial, por lo que el análisis de redundancia parcial permitió evaluar de manera efectiva el ensamble de invertebrados en relación al uso del suelo, considerando el efecto de la vegetación separadamente.

En este trabajo los patrones observados en arroyos hortícolas fueron similares a los registrados en usos de suelo agrícola. Por ejemplo, Malacarne et al. (2016) estudiaron los invertebrados acuáticos y la descomposición de la hojarasca en arroyos con distinto uso del suelo (urbano, agrícola y áreas de bosque preservado) en Brasil, determinando mayores valores de riqueza y abundancia en arroyos de referencia. Consistentemente con este estudio, los taxones de invertebrados sensibles se encontraron sólo en sitios de referencia, siendo la comunidad de invertebrados significativamente diferente de las comunidades de usos de suelo urbano y agrícola. Fierro et al. (2017) estudiaron el ensamble de invertebrados de arroyos de bosque nativo, bosque implantado y uso de suelo agrícola en Chile, determinaron mayores concentraciones de nutrientes y menor riqueza taxonómica y diversidad en cuencas agrícolas comparadas con otros usos de suelo. Si se considera que la horticultura representa un uso más intensivo debido a la diversidad de productos utilizados y por la mayor frecuencia de aplicación, la horticultura puede ser considerada como una forma de agricultura intensiva; ambas prácticas resultan en efectos similares en los arroyos. 
CONCLUSIONES 


\section{CONCLUSIONES}

En el presente trabajo se estudió el impacto de la utilización de agroquímicos en el Cinturón Hortícola Platense a varias escalas de trabajo, organismo, población y comunidad.

Se determinó que Simocephalus vetulus es una de las especies de amplia distribución, abundante en los ambientes regionales, más sensibles a los plaguicidas en comparación con otras especies de crustáceos, particularmente para lambdacialotrina y glifosato. Su tamaño comparativamente grande facilitó su observación y manipulación, características que contribuyen a su utilización como modelo de estudio, mientras que por su sensibilidad resulta de gran utilidad como herramienta para determinar toxicidad. Se concluye que $S$. vetulus presenta características adecuadas para ser considerada como modelo local para la evaluación del impacto por agroquímicos.

Los resultados de los experimentos de exposición a repetidos pulsos efímeros de toxicidad indicaron que una población expuesta a piretroides (lambdacialotrina y cipermetrina) disminuirá su longevidad, tasa de alimentación y fecundidad, resultando en la disminución de su densidad poblacional. Se concluye que distintas especies de invertebrados se verán afectadas de manera diferencial debido a su diferente sensibilidad, resultando en cambios en la estructura del ensamble.

Se confirmó la hipótesis, la utilización de fertilizantes en cuencas hortícolas contribuye al incremento de las concentraciones de nutrientes en las aguas superficiales. Asimismo, la aplicación de plaguicidas en los cultivos incrementa su concentración en los sedimentos de los arroyos adyacentes.

Se confirma la hipótesis que la aplicación de agroquímicos en los cultivos afecta a los ensambles de arroyos adyacentes formados por organismos de diferente sensibilidad, modificando la composición y la estructura del ensamble por pérdida de organismos sensibles y aumento de la densidad de grupos taxonómicos tolerantes. Los arroyos hortícolas se caracterizaron por menor riqueza y densidad, y por ensambles conformados por colémbolos (principalmente Entomobryoidea) y grupos taxonómicos tolerantes como Entomobryioidea, y Dugessidae, mientras que los arroyos de referencia se caracterizaron por su mayor riqueza y densidad y por ensambles con dominancia de grupos sensibles a los plaguicidas: S. vetulus, Hyalella, Baetidae y Caenis. 
Se concluye que $S$. vetulus y Hyalella sp. son buenos indicadores del impacto producido por la horticultura en los arroyos pampeanos, como así también las concentraciones de nutrientes y la riqueza de taxones.

Se confirma la hipótesis general, la aplicación de agroquímicos en el Cinturón Hortícola Platense deteriora la calidad del agua y modifica la estructura de los ensambles de invertebrados de los arroyos adyacentes. La horticultura tal como se desarrolla en el Cinturón Hortícola Platense causa un daño a la biota resultando en una comunidad tolerante con pérdida de diversidad. 


\section{ANEXO}

Lista de nombres completos de las especies mencionadas en apartado 1.3.2 Sensibilidad de Simocephalus vetulus en relación a otros crustáceosdel Capítulo 1 y de las tablas 3.5 y 3.10 del Capítulo 3.

\section{ANOSTRACA}

Streptocephalus (Parastreptocephalus) sudanicus Daday, 1910 (sensu Hamer et al., 1994).

\section{AMPHIPODA}

Gammarus pseudolimnaeus Bousfield, 1958.

G. pulex (L., 1758).

Hyalella azteca (Saussure, 1858).

H. curvispina Shoemaker, 1942.

\section{CLADOCERA}

Ceriodaphnia dubia Richard, 1895.

C. silvestrii (Daday, 1902).

Daphnia ambigua Scourfield, 1947.

D. carinata King, 1852.

D. cucullata Sars, 1862.

D. galeata Sars, 1864

D. longispina Müller, 1776

D. magna Straus, 1820.

D. pulex Leydig, 1860.

Simocephalus daphnoides Herrick 1892.

S. vetulus (O.F. Müller, 1776). 


\section{COPEPODA}

Acartia clausi Giesbrecht, 1889

Phyllodiaptomus (Ctenodiaptomus) annae (Apstein, 1907).

Diaptomus forbesi Light, 1938.

Oithona similis Claus, 1866.

Temora longicornis (Müller O.F., 1785).

\section{OSTRACODA}

Chlamydotheca ihieringi (Sars, 1909).

C. incisa (Claus, 1892).

\section{ISOPODA}

Asellus aquaticus (Linnaeus, 1758).

Proasellus coxalis (Dollfus, 1892).

\section{DECAPODA}

Macrobrachium lanchesteri (de Man, 1911).

M. lar (Fabricius, 1798).

M. roserbergi (de Man, 1879).

Neocaridina denticulata (De Haan, 1844 [in De Haan, 1833-1850]).

Procambarus clarkii (Girard, 1852).

Spiralothelphusa hydrodroma (Herbst, 1794).

Trichodactylus borellianus Nobili, 1896. 


\section{BIBLIOGRAFÍA}

Abdelghani A. A., Tchounwou P. B., Anderson A. C., Sujono C. H., Heyer L. R., Monkiedje A. 1997. Toxicity evaluation of single and chemical mixtures of roundup, Garlon-3A, 2,4-D, and syndets surfactant to channel catfish (Ictalurus punctatus), bluegill sunfish (Lepomis microchirus), and crawfish (Procambarus spp.). Environmental Toxicology and Water Quality 12 (3): 237-243.

Alberdi J. L., Saenz M. E., Di Marzio W. D., Tortorelli M. C. 1996. Comparative acute toxicity of two herbicides, Paraquat and Glyphosate, to Daphnia magna and D. spinulata. Bulletin of Environmental Contamination and Toxicology 57 (2): 229-235.

Allan J. D. 2004. Landscapes and riverscapes: The Influence of Land Use on Stream Ecosystems. Annual Review of Ecology, Evolution, and Systematics 35: 257-84.

Allen, J. A. 1877. The influence of physical conditions in the genesis of species. Radical Review 1: 108-140.

Anguiano O. L., Ferrari A., Soleño J., Martínez M. C., Venturino A., Pechen de Angelo A. M., Montagna C. M. 2008. Enhanced esterase activity and resistance to azinphosmethyl in target and non-target organisms. Environmental Toxicology and Chemistry 27 (10): 2117-2123.

Aparicio V. C., De Gerónimo E., Marino D., Primost J., Carriquiriborde P., Costa J. L. 2013. Environmental fate of glyphosate and aminomethylphosphonic acid in surface waters and soil of agricultural basins. Chemosphere 93 (9): 1866-1873.

APHA, 2012. American Public Health Association.Standard Methods for the Examination of Water and Wastewater, 22nd edition. Washington DC., pp. 1360.

Arbuckle K. E., Downing J. A. 2001. The influence of watershed land use on lake N:P in a predominantly agricultural landscape. Limnology and Oceanography 46 (4): 970-975.

Argerich C., Troilo L. (ed), 2010. Buenas prácticas agrícolas en la cadena de tomate. FAO, INTA. Buenos Aires, Argentina, pp. 258.

Ashauer R., Boxall A. A., Brown C. D. 2007. New ecotoxicological model to simulate survival of aquatic invertebrates after exposure to fluctuating and sequential pulses of pesticides. Environmental Science and Technology 41: 1480-1486.

Athor J. (ed), 2009. Parque Costero del Sur - Naturaleza, conservación y patrimonio cultural. Fundación de Historia Natural "Félix de Azara", Buenos Aires, pp. 528.

Atkinson D. 1994. Temperature and organism size - a biological law for ectoterms? Advances in Ecological Research 25: 1-58.

Atkinson D., Sibly R. M. 1997. Why are organisms usually bigger in colder environments? Making sense of a life history puzzle. Trends in Ecology \& Evolution 12: 235-239. 
Aubone A., Wöhler O. C. 2000. Aplicación del método de máxima verosimilitud a la estimación de parámetros y comparación de curvas de crecimiento de von Bertalanffy. INIDEP informe técnico 37: 1-21.

Bailey H. C., Miller J. L., Miller M. J., Wiborg L. C., Deanovic L., Shed T. 1997. Joint acute toxicity of diazinon and chlorpyrifos to Ceriodaphnia dubia. Environmental Toxicology and Chemistry 16 (11): 2304-2308.

Bajet C. M., Kumar A., Calingacion M. N., Narvacan T. C. 2012. Toxicological assessment of pesticides used in the Pagsanjan-Lumban catchment to selected non-target aquatic organisms in Laguna Lake, Philippines. Agricultural Water Management 106: 42-49.

Balayla D. J., Moss B. 2003. Spatial patterns and population dynamics of plant-associated microcrustacea (Cladocera) in an English shallow lake (Little Mere, Cheshire). Aquatic Ecology 37 (4): 417-435.

Barata C., Baird D. J., Medina M., Albalat A., Soares A. M. V. M. 2002. Determining the ecotoxicological mode of action of toxic chemicals in meiobenthic marine organisms: stage-specific short tests with Tisbe battagliai. Marine Ecology Progress Series 230: 183-194.

Barata C., Baird D. J., Nogueira A. J. A., Soares A. M. V. M., Riva M. C. 2006.Toxicity of binary mixtures of metals and pyrethroid insecticides to Daphnia magna Straus. Implications for multi-substance risks assessment. Aquatic Toxicology 78: 1-14.

Barata C., Alañon P., Gutierrez-Alonso S., Riva M. C., Fernandez C., Tarazona J. V. 2008. A Daphnia magna feeding bioassay as a cost effective and ecological relevant sublethal toxicity test for Environmental Risk Assessment of toxic effluents. Science of the total Environment 405: 78-86.

Barbosa F. A. R., Callisto M., Galdean N. 2001. The diversity of benthic macroinvertebrates as an indicator of water quality and ecosystem health: a case study for Brazil. Aquatic Ecosystem Health and Management 4: 51-59.

Barbour M. T., Gerritsen J., Snyder, B. D., Stribling, J. B., 1999. Rapid bioassessment protocols for use in streams and wadeable rivers: periphyton, benthic macroinvertebrates and fish, 2nd edition. U.S. Environmental Protection Agency, Washington D.C., pp.197.

Barsky A. 2005. El periurbano productivo, un espacio en constante transformación. Introducción al estado del debate, con referencias al caso de Buenos Aires. Scripta Nova, revista electrónica de geografía y ciencias sociales IX (194): 36.

Barsky A. 2010. La agricultura de "cercanías" a la ciudad y los ciclos del territorio periurbano. Reflexiones sobre el caso de la Región Metropolitana de Buenos Aires. En Svetlitzia de Nemirovsky, A. (Coord.) Globalización y agricultura periurbana en la Argentina. Escenarios, recorridos y problemas. Editorial FLACSO. Buenos Aires, pp.15-29. 
Barsky A., Vio M. 2007. La problemática del ordenamiento territorial en cinturones verdes periurbanos sometidos a procesos de valorización inmobiliaria. El caso del Partido del Pilar, Región Metropolitana de Buenos Aires. IX Coloquio Internacional de Geocrítica, Universidad Federal de Rio Grande do Sul, Porto. Alegre. Disponible en: http://www.ub.es/geocrit/9porto/barsky.htm Último acceso: Marzo 2018.

Begon M., Townsend C. R., Harper J. L. 2006. Ecology, from individuals to ecosystems, 4th edition, Blackwell Publishing Ltd, UK. pp. 759.

Beketov M. A., Foit K., Schäfer R. B., Schriever C. A., Sacchi A., Capri E., Biggs J., Wells C., Liess M. 2009. SPEAR indicates pesticide effects in streams - Comparative use of species- and family-level biomonitoring data. Environmental Pollution 157: 1841-1848.

Beketov M. A., Kefford B. J., Schäfer R. B., Liess M. 2013. Pesticides reduce regional biodiversity of stream invertebrates. Proceedings of the National Academy of Science of the United States 110 (27) 11039-11043.

Bengtsson G., Hansson L-A., Montenegro K. 2004. Reduced grazing rates in Daphnia pulex caused by contaminants: implications for trophic cascades. Environmental Toxicology and Chemistry 23 (11): 2641-2648.

Bergmann, C. 1847. Über die Verhältnisse der Wärmeökonomie der Thiere zu ihrer Grösse. Göttinger Studien 3: 595-708.

Berenzen N., Kumke T., Holger K. H., Schulz K., Schulz R. 2005. Macroinvertebrate community structure in agricultural streams: impact of runoff-related pesticide contamination. Ecotoxicology and Environmental Safety 60: 37-46.

Bernava Laborde V., Palacios-Vargas J. G. 2008. Collembola. En Claps L. E., Debandi G. y Roig-Juñent S. (dirs). Biodiversidad de Artrópodos Argentinos 2. Editorial Sociedad Entomológica Argentina. Mendoza. 151-166 pp.

Bertrand L., Monferrán M. V., Mouneyrac C., Amé M. V. 2018. Native crustacean species as a bioindicator of freshwater ecosystem pollution: A multivariate and integrative study of multi-biomarker response in active river monitoring. Chemosphere 206: 265-277.

Boletín Oficial 2014. Resolución № 571/2014. Disponible en: https://www.boletinoficial.gob.ar/ Último acceso: Septiembre 2018.

Boletín Oficial 2018. Decreto 302/17. Disponible en https://www.boletinoficial.gob.ar/ Último acceso: Septiembre 2018.

Brausch J. M., Beall B., Smith P. N. 2007. Acute and sub-lethal toxicity of three POEA surfactant formulations to Daphnia magna. Bulletin of Environmental Contamination and Toxicology 78 (6): 510-514.

Brito D., Milani N., Pereira G. 2006. Tasa de filtración e ingestión de Simocephalus vetulus (Müller, 1776) (Crustacea: Cladoecra) alimentado con Selenastrum capricornutum Printz, 1914 y Chlorella vulgaris Beijernick, 1980. Interciencia 31 (10): 753-757. 
Brix K.V., DeForest D.K., Adams W.J. 2001. Assessing acute and chronic copper risks to freshwater aquatic life using species sensitivity distributions for different taxonomic groups. Environmental Toxicology and Chemistry 20 (8): 1846-1856.

Brusca R. C., Brusca G. J. 2005. Invertebrados, 2da Edición. Editorial Mc GrawHill/Interamericana de España, S. A. U. Madrid, España pp. 1032.

Cáceres T., He W., Naidu R., Megharaj M. 2007. Toxicity of chlorpyrifos and TCP alone and in combination to Daphnia carinata: The influence of microbial degradation in natural water. Water Research 41 (19): 4497-4503.

Camino M., Aparicio V. 2010. Aspectos Ambientales del Uso de Glifosato. Estación Experimental Balcarce. Ediciones INTA, pp. 114.

Caracciolo M. 2016. Situación de la institucionalidad de apoyo a la innovación comercial y de los procesos de gestión comercial de la agricultura familiar en la Argentina. / Mercedes Caracciolo; colaboración de Paula Fontana y Augusto de Haro. Instituto Interamericano de Cooperación para la Agricultura (IICA), Buenos Aires, Argentina pp. 238.

Carpenter S.R., Bolgrien D., Lathrop R.C., Stowe C.A., Reed T., Wilson M.A. 1997. Ecological and economic analysis of lake eutrophication by nonpoint pollution. Australian Journal of Ecology 23: 68-79.

CASAFE, 2013. Cámara de Sanidad Agropecuaria y Fertilizantes, Buenos Aires, Argentina. http://www.casafe.org/publicaciones/estadisticas/ Último acceso: Diciembre 2017.

Castillo Morales G. (ed.) 2004. Ensayos toxicológicos y métodos de evaluación de calidad de aguas. Estandarización, intercalibración, resultados y aplicaciones. México: IMTA.

Cebrián C., Andreu-Moliner E. S., Fernández-Casalderrey A., Ferrando M. D. 1992. Acute toxicity and oxygen consumption in the gills of Procambarus clarkii in relation to chlorpyrifos exposure. Bulletin of Environmental Contamination and Toxicology 49 (1): 145-149.

Cellone F., Tosi L., Carol E. 2018. Estimating the freshwater-lens reserve in the coastal plain of the middle Río de la Plata Estuary (Argentina). Science of the Total Environment 630: 357-366.

César I. I., Ocón C. S., Paggi A. C., Rodrigues Capitulo A., Spacessi F., Tangorra M., Tassara M. P. 2000 Diversidad de invertebrados bentónicos del Río de la Plata. Biología Acuática 19: 27-63.

Chen C. Y., Hathaway K. M., Folt C. L., 2004. Multiple stress effects of Vision® herbicide, pH and food on zooplankton and larval amphibian species from forest wetlands. Environmental Toxicology and Chemistry 23 (4): 823-831.

Chevalier J., Harscoët E., Keller M., Pandard P., Cachot J., Grote, M. 2015. Exploration of Daphnia behavioral effect profiles induced by a broad range of toxicants with different modes of action. Environmental Toxicology and Chemistry, 34(8), 1760-1769. 
CHFB 2005. Censo Horti-Florícola Bonaerense. Ministerio de Asuntos Agrarios y Ministerio de Economía, Secretaria de Agricultura y Ganadería. http://www.estadistica.ec.gba.gov.ar/dpe/Estadistica/chfba/censohort.htm Último acceso: Abril 2018.

Christensen B. T., Lauridsen T. L., Ravn H. W., Bayley M. 2005. A comparison of feeding efficiency and swimming ability of Daphnia magna exposed to cypermethrin. Aquatic Toxicology 73: 210-220.

CIAFA 2017. Cámara de la Industria Argentina de Fertilizantes y Agroquímicos. Informe sobre evolución de la agricultura y uso de fertilizantes. http://www.ciafa.org.ar/info-fertilizantesinformes Último acceso: Diciembre 2017.

Clarke K. R., Gorley R. N. 2001. PRIMER v5: User Manual/Tutorial. PRIMER-E, Plymouth, pp. 192.

Clarke K. R., Warwick R. M. 2001.Change in marine communities: an approach to statistical analysis and interpretation, 2nd edition. PRIMER-E: Plymouth, pp. 172.

Cold A., Forbes V. E. 2004. Consequences of a short pulse of pesticide exposure for survival and reproduction of Gammarus pulex.Aquatic Toxicology 67: 287-299.

Colombo J. C, Astoviza M., Bilos C., Cappelletti N., Migoya C., Morrone M., Skorupka C., Speranza E., Tatone L., 2015. Biogeoquímica de contaminantes. En Relevamiento de la utilización de Agroquímicos en la Provincia de Buenos Aires - Mapa de Situación e incidencias sobre la salud. Defensoría del Pueblo de la Provincia de Buenos Aires. pp. 497-532.

Cooper C. M. 1993. Biological Effects of Agriculturally Derived Surface Water Pollutants on Aquatic Systems—A Review. Journal of Environment Quality 22 (3): 402-408.

Cortelezzi A., Ocón C., López van Oosterom M. V., Cepeda R., Rodrigues Capítulo A. 2015. Nutrient enrichment effect on macroinvertebrates in a lowland stream of Argentina. Ilheringia, Série Zoologia 105 (2): 228-234.

Cuhra M., Traavik T., Bøhn T. 2013. Clone- and age-dependent toxicity of a glyphosate commercial formulation and its active ingredient in Daphnia magna. Ecotoxicology 22: 251-262.

Currie Z., Prosser R. S., Rodriguez-Gil J. L., Mahon K., Poirier D., Solomon K. R. 2015. Toxicity of Cúspide $480 S L \otimes$ spray mixture formulation of glyphosate to aquatic organisms. Environmental Toxicology and Chemistry 34 (5): 1178-1184.

Daniels R. E., Allan J. D. 1981. Life Table Evaluation of Chronic Exposure to a Pesticide. Canadian Journal of Fisheries and Aquatic Sciences 38 (5): 485-494.

Das P., Mandal S. C., Bhagabati S. K., Akhtar M. S., Singh S. K. 2012. Important live food organisms and their role in aquaculture. Frontiers in Aquaculture pp.69-86. 
Day K., Kaushik N. K. 1987. An Assessment of the Chronic Toxicity of the Synthetic Pyrethroid, Fenvalerate, to Daphnia galeata mendotae, using Life Tables. Environmental Pollution 44: 13-26.

Deepananda A. K. H. M., Gajamange D., De Silva W.A.J.P., Wegiriya H.C.E. 2001. Acute toxicity of a glyphosate herbicide, Roundup $®$, to two freshwater crustaceans. Journal of the National Science Foundation of Sri Lanka 39 (2): 169-173.

Defensoría del Pueblo 2018. Documento diagnóstico. Arrendamientos rurales en el Partido de La Plata. Mesa de Trabajo. Exp 22800-18933/2018. Defensoría del Pueblo de la Provincia de Buenos Aires, Argentina pp. 15.

Demetrio P. M. 2012. Estudio de efectos biológicos de plaguicidas utilizados en cultivos de soja RR y evaluación de impactos adversos en ambientes acuáticos de agroecosistemas de la región pampeana. Tesis Doctoral, Facultad de Ciencias Exactas, Universidad Nacional de La Plata pp. 156.

Demetrio P. M., Bonetto C., Ronco A. E. 2014.The effect of Cypermethrin, Chlorpyrifos, and Glyphosate active ingredients and formulations on Daphnia magna (Straus). Bulletin of Environmental Contamination and Toxicology 93: 268-273.

Dethier M. N., Graham E. S., Cohen S., Tear L. M., 1993. Visual versus random-point percent cover estimations: 'objective' is not always better. Marine Ecology Progress Series 96 (1): 93-100.

Domínguez E., Fernández H.R. (eds), 2009. Macroinvertebrados bentónicos. Sistemática y Biología. $1^{\text {a }}$ edición. Fundación Miguel Lillo, Tucumán, pp. 656.

Dudgeon D., Arthington A. H., Gessner M. O., Kawabata Z.-I., Knowler D. J., Levêque C., Robert J. Naiman R. J., Prieur-Richard8 A.-H., Soto D., Stiassny M. L. J., Sullivan C. A., 2006. Freshwater biodiversity: importance, threats, status and conservation challenges. Biological Reviews 81: 163-182.

Duré S. 2014. Caracterización de estrategias comerciales de pequeños productores del cinturón hortícola del Gran La Plata. Análisis a partir de estudios de caso. Boletín Hortícola 18 (51): 13-23.

Ebert D., 1991.The effect of size at birth, maturation thresholds and genetic differences on the life-history of Daphnia magna.Oecologia 86: 243-250.

ECOTOX, 2000. Ecotoxicology Database from U. S. Enviromental Protection Agency. Versión Actualizada: 2014. Disponible en http://cfpub.epa.gov/ecotox Último acceso: Septiembre 2018.

Egler M. A., Buss D. F., Moreira J. C., Baptista D. F. 2012. Influence of agricultural landuse and pesticides on benthic macroinvertebrate assemblages in an agricultural river basin in southeast Brazil. Brazilian Journal of Biology 72: 437-443. 
Endlweber K., Schädler M., Scheu S. 2006. Effects of foliar and soil insecticide applications on the collembolan community of an early set-aside arable field.Applied Soil Ecology 31: 136-146

Fernández Lozano J. 2012. La producción de hortalizas en Argentina. Corporación del Mercado Central de Buenos Aires. Secretaría de Comercio Interior, pp .29.

Ferrando M.D., Janssen C.R., Andreu E., Persoone G. 1993. Ecotoxicological studies with the freshwater rotifer Brachionus calyciflorus II. An assessment of the chronic toxicity of lindane and 3,4-dichloroaniline using life tables. Hydrobiologia 255 (1): 33-40.

Ferrando N. S. 2015. Zooplancton de ambientes acuáticos de la cuenca del río Salado (Buenos Aires): estudio de relaciones interespecíficas y principales factores de control mediante experiencias de laboratorio y microscosmos. Tesis Doctoral. Facultad de Ciencias Naturales y Museo, Universidad Nacional de la Plata. 216pp.

Ferreiro N., Feijoo C., Giorgi A., Rosso J., 2014. Macroinvertebrates select complex macrophytes independently of their body size and fish predation risk in a Pampean stream. Hydrobiologia 740 (1): 191-205.

Fierro P., Bertrán C., Tapia J., Hauenstein E., Peña-Cortés F., Vergara C., Cerna C., VargasChacoff L., 2017. Effects of local land-use on riparian vegetation, water quality, and the functional organization of macroinvertebrate assemblages. Science of the Total Environment 609: 724-734.

Figueiredo R. O., Markewitz D., Davidson E. A., Schuler A. E., Watrin O. S., de Souza Silva P. 2010. Land-use effects on the chemical attributes of low-order streams in the eastern Amazon. Journal of Geophysical Research. 115 (G4).

Figueroa R., Valdovinos C., Araya E., Parra O. 2003. Macroinvertebrados bentónicos como indicadores de calidad de agua de ríos del sur de Chile. Revista Chilena de Historia Natural 76: 275-285.

Fonseca A. L., Rocha O., 2004. The life cycle of Ceriodaphnia silvestrii Daday 1902, a Neotropical endemic species (Crustacea, Cladocera, Daphnidae). Acta Limnologica Brasilera 16 (4): 319-328.

Forbes V. E., Calow P. 1999. Is the per capita rate of increase a good measure of populationlevel effects in ecotoxicology? Environmental Toxicology and Chemistry 18 (7): 15441556 .

Forró L., Korovchinsky N. M., Kotov A. A., Petrusek A., 2008. Global diversity of cladocerans (Cladocera; Crustacea) in freshwater. Hydrobiologia 595: 177-184.

Fryer G., 1987. Morphology and the classification of the so-called Cladocera.Hydrobiologia 145: 19-28. 
García M. 2010. Inicios, consolidación y diferenciación de la horticultura platense. En Svetlitzia de Nemirovsky, A. (Coord.), Globalización y agricultura periurbana en la Argentina. Escenarios, recorridos y problemas. Editorial FLACSO, Buenos Aires. pp. 69-85.

García M. 2011. La producción platense en el marco de su zona circundante (AHB), Boletín Hortícola, 16 (47): 7-13.

García M., Lemmi S. 2011. Política legislativa y trabajo en la horticultura del Área Metropolitana de Buenos Aires (Argentina). Orígenes y continuidades de la precarización laboral en la horticultura. Secuencia 79: 89-112.

García M. 2012. Análisis de las transformaciones de la estructura agraria hortícola platense en los últimos 20 años. El rol de los horticultores bolivianos. Tesis Doctoral, Facultad de Ciencias Agrarias y Forestales, Universidad Nacional de La Plata, pp. 432.

García M. 2014. La renta en la horticultura de La Plata (Buenos Aires, Argentina). Causas de su heterogeneidad intra y extrarregional. Agroalimentaria 20 (38): 107-120.

García M. 2015. Horticultura de La Plata (Buenos Aires). Modelo productivo irracionalmente exitoso. Revista de la Facultad de Agronomía 114 (Esp.1 Agricultura Familiar, Agroecología y Territorio): 190-201.

García S. I. y Lazovski, J. 2011. Guía de Uso Responsable de Agroquímicos. 1ra edición. Ministerio de Salud de la Nación. Programa Nacional de Prevención y Control de las Intoxicaciones. Buenos Aires pp. 36.

Gerhardt A. 2002. Bioindicator species and their use in biomonitoring. En UNESCO, editor. Encyclopedia of life support systems.EOLSS, Oxford pp. 50.

Giesy J. P., Dobson S., Solomon K. R. 2000. Ecotoxicological Risk Assessment for Roundup ${ }^{\circledR}$ Herbicide. Reviews of Environmental Contamination and Toxicology 167: 35-120.

Giorgi A., Feijoo C., Tell G. 2005. Primary producers in a Pampean stream: temporal variation and structuring role. Biodiversity and Conservation 14: 1699-1718.

Gliwicz M., Sieniawska A.1986. Filtering activity of Daphnia in low concentrations of a pesticide. Limnology and Oceanography 31 (5): 1132-1138.

Gliwicz, Z. M., Boavida, M. J. 1996. Clutch size and body size at first reproduction in Daphnia pulicaria at different levels of food and predation. Journal of Plankton Research 18 (6): 863-880.

Gómez N., Rodriguez Capitulo A., Colautti D., Mariñelarena A. Licursi M., Cochero J., Armendariz L., Maroñas M., Donadelli J., Jensen R., García de Souza J., Maiztegui T., García I., Sathicq M. B., Suarez J., Cortese B. (2016) La puesta en valor de los servicios ecosistémicos que ofrecen los arroyos de llanura como una medida de mitigación de las inundaciones: el caso del $\mathrm{A}^{\circ}$ del Gato en el Partido de La Plata. En Ecología y manejo de ecosistemas acuáticos pampeanos. Volpedo A. V., de Cabo L., Arreghini S., Fernández Cirelli A., (eds.). Buenos Aires, 39-52. 
Gordo T., Lubián L. M., Cañavate J. P., 1994. Influence of temperature on growth, reproduction and longevity of Moina salina Daday, 1888 (Cladocera, Moinidae).Journal of Plankton Research 6 (11): 1513-1523.

Graca M. A. S., Rodrigues-Capítulo A., Ocón C., Gomez N. 2002. In situ tests for water quality assessment: a case study in Pampean rivers. Water Research 36: 4033-4040.

Gücker B., Boëchat I. G., Giani A., 2009. Impacts of agricultural land use on ecosystem structure and whole-stream metabolism of tropical Cerrado streams. Freshwater Biology 54: 2069-2085.

Guisande C., Gliwicz Z. M. 1992. Egg size and clutch size in two Daphnia species grown at different food levels. Journal of Plankton Research 14 (7): 997-1007.

Güntzel A. M., Matsumura-Tundisi T., Rocha O., 2003. Life cycle of Macrothrix flabelligera Smirnov, 1992 (Cladocera, Macrothricidae), recently reported in the Neotropical region. Hydrobiologia 490 (1-3): 87-92.

Gutierrez M. F., Gagneten A. M., Paggi J. C. 2012. Exposure to sublethal chromium and endosulfan alter the diel vertical migration (DVM) in freshwater zooplankton crustaceans. Ecotoxicology 21 (1): 37-47.

Gutierrez M. F., Negro C. L. 2014. Predator-prey imbalances due to a pesticide: density and applicability timing as determining factors for experimental assessments. Ecotoxicology 23 (7): 1210-1219.

Hanazato T. 2001. Pesticide effects on freshwater zooplankton: an ecological perspective. Environmental Pollution 112 (1): 1-10.

Harmon S. M., Specht W. L., Chandler G. T. 2003. A comparison of the daphnids Ceriodaphnia dubia and Daphnia ambigua for their utilization in routine toxicity testing in the southeastern United States. Archives of Environmental Contamination and Toxicology 45 (1): 79-85.

Harrison P. A., Vandewalle M., Sykes M. T., Berry P. M., Bugter R., de Bello F., Feld C. K., Grandin U., Harrington R., Haslett J. R., Jongman R. H. G., Luck G., Martins da Silva P., Moora M., Settele J., Sousa J. P., Zobel M. 2010. Identifying and prioritising services in European terrestrial and freshwater ecosystems. Biodiversity and Conservation 19 (10): 2791-2821.

Hart M. R., Quin B. F., Nguyen M. L., 2004. Phosphorus runoff from agricultural land and direct fertilizer effects: a review. Journal of Environmental Quality 33: 1954-1972.

Hebert P. D. N. 1978. The population biology of Daphnia (Crustacea, Daphnidae). Biology Reviews 53: 387-426.

Henry C. J., Higgins K. F., Buhl K. J. 1994. Acute toxicity and hazard assessment of Rodeo®, X-77 Spreader $\circledast$, and Chem-Trol ${ }^{\circledR}$ to aquatic invertebrates. Archives of Environmental Contamination and Toxicology 27 (3): 392-399. 
Hose G. C. 2005. Assessing the need for groundwater quality guidelines for pesticides using the species sensitivity distribution approach. Human and Ecological Risk Assessment 11 (5): 951-966.

Hunt L., Bonetto C., Resh V. H., Buss D. F., Fanelli S., Marrochi N., Lydy M. J. 2016. Insecticide concentrations in stream sediments of soy production regions of South America. Science of the Total Environment 547: 114-124.

Hunt L., Bonetto C., Marrochi N., Scalise A., Fanelli S., Liess M., Lydy M., Chiu C., Resh V. H. 2017. Species at Risk (SPEAR) index indicates effects of insecticides on stream invertebrate communities in soy production regions of the Argentine Pampas. Science of the Total Environment 580: 699-709.

Hurtado M. A., Gimenez J.E., Cabral M.G. (eds) 2006. Análisis ambiental del partido de La Plata: Aportes al ordenamiento territorial, 1ra edición. Buenos Aires, Consejo Federal de Inversiones pp. 125.

IGN 2015. Instituto Geográfico Nacional, Información geoespacial. Disponible en: http://www.ign.gob.ar/NuestrasActividades/InformacionGeoespacial/CapasSIG Último acceso: Febrero 2018.

Infoleg 2015. Ley 27.118. Reparación histórica de la Agricultura Familiar para la construcción de una nueva ruralidad en la Argentina. Disponible en: http://servicios.infoleg.gob.ar/infolegInternet/anexos/240000-244999/241352/norma.htm Último Acceso: Septiembre 2018.

Jergentz S., Mugni H., Bonetto C., Schulz R. 2004. Runoff-related endosulfan contamination and aquatic macroinvertebrate response in rural basins near Buenos Aires, Argentina. Archives of Environmental Contamination and Toxicology 46 (3): 345-353.

Jergentz S., Mugni H., Bonetto C., Schulz R. 2005. Assessment of insecticide contamination in runoff and stream water of small agricultural streams in the main soybean area of Argentina. Chemosphere 61 (6): 817-826.

Jobbágy E. 2011. Servicios Hídricos de los Ecosistemas y su Relación con el uso de la tierra en la Ilanura Chaco-Pampeana. En Laterra P., Jobaggy E. G., Paruelo J. M. (eds). Valoración de Servicios Ecosistemicos. Ediciones INTA, 409-442.

Jones D. 1995. Environmental Fate of Cypermethrin. Environmental Monitoring and Pest management Branch, Department of Pesticide Regulation, Sacramento, California, USA.

Jordan T. E., Correll D. L., Weller D. E. 1997. Relating nutrient discharges from watersheds to land use and streamflow variability. Water Resources Research 33 (11): 2579-2590.

Kahl M. B. 2013. Principales características de los insecticidas utilizados en el cultivo de soja. INTA, Estación Experimental Agropecuaria Paraná. Serie Extensión Digital, Segundo Trimestre 5: 31-50. 
Kammerbauer J., Moncada J., 1998. Pesticide residue assessment in three selected agricultural production systems in the Choluteca River Basin of Honduras. Environmental Pollution 103: 171-181.

Kashian D. R., Dodson S. I. 2002. Effects of common-use pesticides on developmental and reproductive processes in Daphnia. Toxicology and Industrial Health 18: 225-235.

Keesstra S. D., van Huissteden J., Vandenberghe J., Van Dam O., de Gier J., Pleizier I. D. 2005. Evolution of the morphology of the river Dragonja (SW Slovenia) due to land-use changes. Geomorphology 69: 191- 207.

Kersting K., van Wijngaarden R. 1992. Effects of chlorpyrifos on a microecosystem. Environmenral Toxicology and Chemistry 11: 365-372.

Kim Y., Jung J., Oh. S., Choi K. 2008. Aquatic toxicity of cartap and cypermethrin to different life stages of Daphnia magna and Oryzias latipes. Journal of Environmental Science and Health, Part B 43 (1): 56-64.

Knauer K., Homazava N., Junghans M., Werner I. 2017. The influence of particles on bioavailability and toxicity of pesticides in surface water. Integrated Environmental Assessment and Management 13 (4): 585-600.

Kreuger J., Graaf S., Patring J., Adielsson S. 2008. Pesticides in surface water in areas with open ground and greenhouse horticultural crops in Sweden. Ekohydrologi 117 pp.49.

Lahr J., Badji A., Marquenie S., Schuiling E., Ndour K. B., Diallo A. O., Everts J. W. 2001. Acute toxicity of locust insecticides to two indigenous invertebrates from sahelian temporary ponds. Ecotoxicology and Environmental Safety 48: 66-75.

Langhammer J. 2003. Antropogenic transformation of River Network in the Otava River Basin. Acta Universitatis Carolinae 2: 139-155.

Laurencena P., Deluchi M., Rojo A., Kruse E. 2008. Influencia de la explotación de aguas subterráneas en el sector periurbano de La Plata. Actas XVII Congreso Geológico Argentino. San Salvador de Jujuy. Tomo II: 472-473.

Leps J., Smilauer P. 2003. Multivariate Analysis of Ecological Data using CANOCO. Cambridge University Press, UK. pp. 283.

Liess M., von der Ohe P.C. 2005. Analyzing effects of pesticides on invertebrate communities in streams. Environmental Toxicology and Chemistry 24 (4): 954-965.

Liess M., Schäfer R.B., Schriever C.A. 2008. The footprint of pesticide stress in communitiesspecies traits reveal community effects of toxicants. Science of the Total Environment 406: 484-490.

Lopes I., Moreira-Santos M., Rendón-von Osten J., Baird D. J., Soares A.M.V.M., Ribeiro R. 2011. Suitability of five cladoceran species from Mexico for in situ experimentation. Ecotoxicology and Environmental Safety 74: 111-116. 
Lorenzen C. J. 1967. Determination of chlorophyll and phaeopigments: spectrophotometric equations. Limnology and Oceanography 12: 343-346.

Mac Loughlin T. M., Peluso L., Marino D. 2017. Pesticide impact study in the peri-urban horticultural area of Gran La Plata, Argentina. Science of the Total Environment 598: 572-580.

Magurran A. E., McGill B. J. (eds) 2011. Biological Diversity: frontiers in measurements and assessment. Oxford University Press, NY. pp. 359.

Malacarne T. J., Baumgartner M. T., Moretto Y., Gubiani É. A. 2016. Effects of Land Use on the Composition and Structure of Aquatic Invertebrate Community and Leaf Breakdown Process in Neotropical Streams. River Research and Applications 32: 1958-1967.

Mañas F., Peralta L., Raviolo J., García Ovando H., Weyers A., Ugnia L., Gonzalez Cid M., Larripa I., Gorla N. 2009. Genotoxicity of AMPA, the environmental metabolite of glyphosate, assessed by the Comet assay and cytogenetic tests. Ecotoxicology and Environmental Safety 72: 834-837.

Mangiafico S. S., Newman J., Merhaut D. J., Gan J., Faber B., Laosheng W. 2009. Nutrients and pesticides in stormwater runoff and soil water in production nurseries and citrus and avocado groves in California. Hort Technology 19 (2): 360-367. HORTICOLA

Manual Fitosanitario 2018. Cámara de la Industria Argentina de Fertilizantes y Agroquímicos (CIAFA). Disponible en http://www.manualfitosanitario.com/ Último acceso: Agosto 2018.

Marino D., Ronco A. E. 2005. Cypermethrin and chlorpiryfos concentration levels in surface water bodies of the Pampa Ondulada, Argentina. Bulletin of Environmental Contamination and Toxicology 75(4): 820-826.

Marrochi M. N. 2018. Impacto de agroquímicos sobre los ensambles de macroinvertebrados en arroyos rurales. Tesis Doctoral. Facultad de Ciencias Naturales y Museo, Universidad Nacional de La Plata pp. 150.

Martínez-Jerónimo F. 2012. Description of the individual growth of Daphnia magna (Crustacea: Cladocera) through the von Bertalanffy growth equation. Effect of photoperiod and temperature. Limnology 13: 65-71.

Martínez-Jerónimo F., Arzate Cárdenas M., Butrón R. O. 2013. Linking sub-individual and population level toxicity effects in Daphnia schoedleri (Cladocera: Anomopoda) exposed to sublethal concentrations of the pesticide a-cypermethrin. Ecotoxicology 22: 985-995.

Mauchly J. W. 1940. Significance Test for Sphericity of a Normal n-Variate Distribution. The Annals of Mathematical Statistics 11 (2): 204-209.

Maund S. J., Hamer M. J., Lane M. C. G., Farrelly E., Rapley J. H., Goggin U. M., Gentile W. E. 2002. Partitioning, bioavailability, and toxicity of the pyrethroids insecticide cypermethrin in sediments. Environmental Toxicology and Chemistry 21 (1): 9-15. 
Mc Mahon J. 2016. Ilustración de Daphnia pulex. Disponible en: https://news.illinois.edu/view/6367/385652. Último acceso: Agosto 2018.

Mc William R., Baird D. J. 2002. Post-exposure feeding depression: a new toxicity endpoint for use in laboratory studies with Daphnia magna. Environmental Toxicology and Chemistry 21 (6): 1198-1205.

Mc Laughlin P. 1980. A Comparative morphology of Recent Crustacea. Editorial W. H. Freeman and Company. San Francisco, California EE.UU. pp. 177.

Merrit R.W., Cummins K.W., Berg M.B. (Eds.) 2008. An Introduction to the Aquatic Insects of North America. Kendall-Hunt, Dubuque, lowa EE.UU. pp. 1158.

Metcalfe T. L., Metcalfe C. D. 1997. The trophodynamics of PCBs including mono and nonortho congeners in the food web of north-Central Lake Ontario. Science of the Total Environment 201: 245-72.

Meyer J. L., Strayer D. L., Wallace J. B., Eggert S. L., Helfman G. S., Leonard N. E. 2007. The contribution of headwater streams to biodiversity in river networks. Journal of the American Water Resources Association 43 (1): 86-103.

Miglioranza K. S. B., Aizpún de Moreno J. E., Moreno V. J. 2003. Dynamics of organochlorine pesticides in soils from a southeastern region of Argentina. Environmental Toxicology and Chemistry 22: 712-717.

Miserendino M. L., Pizzolón L.A 1999. Rapid Assessment of River Water Quality using macroinvertebrates: a Family Level Index for the Patagonian Andean zone. Acta Limnologica Brasilera 2 (2): 137-148.

Miserendino M. L., Casaux R., Archangelsky M., Di Prinzio C. Y., Brand C., Kutschker A. M. 2011. Assessing land-use effects on water quality, in-stream habitat, riparian ecosystems and biodiversity in Patagonian northwest streams. Science of the Total Environment 409: 612-624.

Mokry L. E., Hoagland K. D. 1990. Acute toxicities of five synthetic pyrethroid insecticides to Daphnia magna and Ceriodaphnia dubia. Environmental Toxicology and Chemistry 9 (8): 1045-1051.

Molina M. C., Roa-Fuentes C. A., Zeni J. O., Casatti L. 2017. The effects of land use at different spatial scales on instream features in agricultural streams. Limnologica 65: 14-21.

Moltoni L. 2012. Evolución del mercado de herbicidas en Argentina. Economía y Desarrollo Agroindustrial. INTA - Instituto de Ingeniería Rural 1 (2): 1-6.

Monsanto 2018. Monsanto Argentina S.A.I.C. Hoja de seguridad de Roundup Full II. Pp. 5. Disponible en: http://www.roundupreadyplus.com.ar/productos Último acceso: Agosto 2018. 
Moore M. T., Huggett D. B., Gillespie Jr. W. B., Rodgers Jr. J. H., Cooper C. M. 1998. Comparative toxicity of Chlordane, Chlorpyrifos, and Aldicarb to four aquatic testing organisms. Archives of Environmental Contamination and Toxicology 34: 152-157.

Moreyra A., Puricelli M., Mercader A., Rey M. I., Córdoba J., Marsans N. 2012. El acceso al agua de los agricultores familiares de la región pampeana: un análisis multidimensional. Mundo Agrario 12 (24).

Morolli C., Quaglio F., Della Rocca G., Malvisi J., Di Salvo A. 2006. Evaluation of the toxicity of synthetic pyrethroids to Red swamp crayfish (Procambarus clarkii, Girard 1852) and common carp (Cyprinus carpio, L. 1758). Bulletin français de la pêche et de la pisciculture 380-381: 1381-1394.

Moss B. 2008. Water pollution by agriculture. Philosophycal Transactions of the Royal Society B 363: $659-666$.

MTE Rural 2017. Movimiento de los Trabajadores Excluídos. http://mteargentina.org.ar/\%E2\%80\%8Bmultitudinario-cebollazo-sur-la-provincia-buenosaires/ Último acceso: Agosto 2018.

Mugni H., Demetrio P., Marino D., Ronco A., Bonetto C. 2010. Toxicity persistence following an experimental cypermethrin and chlorpyrifos application in pampasic surface waters (Buenos Aires, Argentina). The Bulletin of Environmental Contamination and Toxicology 84: $524-528$.

Mugni H., Ronco A., Bonetto C. 2011. Insecticide toxicity to Hyalella curvispina in runoff and stream water within a soybean farm (Buenos Aires, Argentina). Ecotoxicology and Environmental Safety 74: 350- 354.

Mugni H., Paracampo A., Marrochi N., Bonetto C. 2013 (a). Acute toxicity of cypermethrin to the non-target organism Hyalella curvispina. Environmental Toxicology and Pharmacology 35: 88-92.

Mugni H., Paracampo A., Bonetto C. 2013 (b). Nutrient concentrations in a pampasic first order stream with different land uses in the surrounding plots (Buenos Aires, Argentina). The Bulletin of Environmental Contamination and Toxicology 91: 391-395.

Mugni H., Paracampo A., Solis M., Fanelli S., Bonetto C. 2015. Acute toxicity of roundup to the nontarget organism Hyalella curvispina. Laboratory and field study. Toxicological \& Environmental Chemistry 96 (7): 1054-1063.

Murugan N., Sivaramakrishnan K. G. 1973. The biology of Simocephalus acutirostratus King (Cladocera: Daphnidae) - laboratory studies of life span, instar duration, egg production, growth and stages in embryonic development. Freshwater Biology 3: 77-83.

Naddy R. B., Johnson K. A., Klaine S. J. 2000. Response of Daphnia magna to pulsed exposures of chlorpyrifos. Environmental Toxicology and Chemistry 19 (2): 423-431. 
Nebeker A. V., Miller C. E. 1989. Use of the amphipod crustacean Hyalella azteca in freshwater and estuarine sediment toxicity tests. Environmental Toxicology and Chemistry 7 (12):1027-1033.

Neter J., Kutner M., Nachtsheim C., Wasserman W., 1996. Applied Linear Regression Models (4th Ed). Editorial McGraw-Hill//rwin. New York. pp. 318.

OECD 1998. OECD Guideline for testing of Chemicals- Daphnia magna reproduction test. Experimental Guideline, Organization for Economic Co-operation and Development, Paris, pp. 25.

OECD 2000. OECD Guideline for testing of Chemicals- Daphnia magna immobilisation test. Experimental Guideline, Organization for Economic Co-operation and Development, Paris, pp. 12.

Olesen J. 2000. An updated phylogeny of the Conchostraca-Cladocera clade (Branchiopoda, Diplostraca). Crustaceana 73 (7): 869-886.

Oliva A., Fernandez L. A., Bachmann A. O. 2002. Sinopsis de los Hydrophiloidea Acuáticos de la Argentina (Insecta, Coleoptera). Monografias del Museo Argentino de Ciencias Naturales 2, Buenos Aires pp. 67.

Oliveira C., Almeida J., Guilhermino L., Soares A. M. V. M., Gravatoa C. 2012. Acute effects of deltamethrin on swimming velocity and biomarkers of the common prawn Palaemon serratus. Aquatic Toxicology 124-125: 209-216.

Olivier H., Lotter A. F., Lemcke G. 2001. Loss on ignition as a method for estimating organic and carbonate content in sediments: reproducibility and comparability of results. Journal of Paleolimnology 25: 101-110.

Olvera-Hernandez E., Martinez Tabche L., Martinez-Jeronimo F. 2004. Bioavailability and Effects of Malathion in Artificial Sediments on Simocephalus vetulus (Cladocera: Daphniidae). he Bulletin of Environmental Contamination and Toxicology 73: 197-204.

Orlinskiy P., Münze R., Beketov M., Gunold R., Paschke A., Knillmann S., Liess M. 2015. Forested headwaters mitigate pesticide effects on macroinvertebrate communities in streams: Mechanisms and quantification. Science of the Total Environment 524-525: 115-123.

Orlova-Bienkowskaya M. J. 1998. A revision of the cladoceran genus Simocephalus (Crustacea: Daphniidae). Bulletin of the Natural History Museum Zoology 64 (1): 1-62.

Ostera J.M., Malanga G., Puntarulo S. 2016. Actualización sobre aspectos oxidativos del efecto del glifosato en sistemas biológicos. Biotecnia, Revista de Ciencias Biológicas y de la Salud 18 (2): 1-10.

Pacheco R. M., Barbona E. I. 2017. Manual de uso seguro y responsable de agroquímicos en cultivos frutihortícolas (1a edición). Ediciones INTA. Bella Vista, Corrientes, Argentina pp. 50. 
Paggi, J. C. 1995. Crustacea Cladocera. En Lopretto E. y Tell G. Ecosistemas de aguas continentales. Ediciones Sur pp. 1401.

Palmer M. A., Allan J. D., Burman C. A. 1996. Dispersal as a regional process affecting the local dynamics of marine and stream benthic invertebrates. Trends in Ecology and Evolution 11 (8): 322-326.

Paracampo A. H., Mugni H.D., Demetrio P. M., Pardi M. H., Bulus G. D., Asborno M. D., Bonetto C. A. 2012. Toxicity persistence in runoff and soil from experimental soybean plots following insecticide applications. Journal of Environmental Science and Health, part B 47: 761-768.

Peluso M. L., Abelando M., Apartin C. D., Almada P., Ronco A. E. 2013. Integrated ecotoxicological assessment of bottom sediments from the Paraná basin, Argentina. Ecotoxicology and Environmental Safety 98: 179-186.

Peluso L., Giusto A., Bulus Rossini G. D., Ferrari L., Salibián A., Ronco A. E., 2011. Hyalella curvispina (Amphipoda) as a test organism in laboratory toxicity testing of environmental samples. Fresenius Environmental Bulletin 20: 372-376.

Perez S., Rial D., Beiras R. 2015. Acute toxicity of selected organic pollutants to saltwater (mysid Siriella armata) and freshwater (cladoceran Daphnia magna) ecotoxicological models. Ecotoxicology 24 (6): 1229-1238.

Perrin N., Ruedi M., Saiah H., 1987. Why is the cladoceran Simocephalus vetulus (Müller) not a "bang-bang strategist"? A critique of the optimal body-size model. Functional Ecology 1 : 223-228.

Perrin N. 1988. Why are offspring born larger when it is colder? Phenotypic plasticity for offspring size in the cladoceran Simocephalus vetulus (Müller). Functional Ecology 2: 283-288.

Perrin N. 1989. Population Density and Offspring Size in the Cladoceran Simocephalus vetulus (Müller).Functional Ecology 3: 29-36.

Peruzzo P. J., Porta A. A., Ronco A. E. 2008. Levels of glyphosate in surface waters, sediments and soils associated with direct sowing soybean cultivation in north pampasic region of Argentina. Environmental Pollution 156: 61-66.

Peters K., Bundschuh M., Schäfer R. B. 2013. Review on the effects of toxicants on freshwater ecosystem functions. Environmental Pollution 180: 324-329.

Pianka E. R. 1982. Ecología Evolutiva. Ediciones Omega, Barcelona pp. 356.

Pórfido O. D. 2014. Los plaguicidas en la República Argentina, 1a edición. Ministerio de Salud de la Nación, Buenos Aires pp. 192.

Posthuma, L., Suter II G. W., Traas T. P. 2002. Species Sensitivity Distributions in Ecotoxicology. Lewis Publishers, Boca Raton, FL. pp. 587. 
QGIS 2015. Quantum GIS, Sistema de Información Geográfico. Disponible en: https://www.qgis.org/es/site/ Último acceso: Febrero 2018.

Radhakrishnaiah K., Renukadevi B. 1990. Size and sex related tolerance to pesticides in the freshwater field crab Oziotelphusa sensex sensex. Environment \& Ecology 8 (1): 111114.

Regaldo I., Reno U., Gervasio S, Troiani H., Gagneten A. M. 2014. Metals effects on Daphnia magna and cladocerans representatives of the Argentinean Fluvial Littoral. Journal of Environmental Biology 35: 689-697.

Reigart J. R., Roberts J. R. 1999. Reconocimiento y manejo de los envenenamientos por pesticidas, 5ta Edición. U.S Environmental Protection Agency, Washington, DC. pp. 252.

Relyea R., Hoverman J. 2006. Assessing the ecology in ecotoxicology: a review and synthesis in freshwater systems. Ecology Letters 9: 1157-1171.

Reno U., Gutierrez M. F., Regaldo L., Gagneten A. M. 2014. The Impact of Eskobat, a Glyphosate Formulation, on the Freshwater Plankton Community. Water Environment Research 86 (12): 2294-2300.

Resh V. H., Norris R. H., Barbour M. T. 1995. Design and implementation of rapid assessment approaches for water resource monitoring using benthic macroinvertebrates. Austral Ecology 20 (1): 108-121.

Richards R. P., Baker D. B. 1993. Pesticide concentration patterns in agricultural drainage networks in the Lake Erie Basin. Environmental Toxicology and Chemistry 12: 13-26.

Rimoldi F., Peluso L., Bulus Rossini, G., Ronco E. A., Demetrio P. M., 2018. Multidisciplinary approach to a study of water and bottom sediment quality of streams associated with mixed land uses: Case study Del Gato Stream, La Plata (Argentina). Ecological indicators 89: 188-198.

Ríos-Touma B., Acosta R., Prat N. 2014. The Andean Biotic Index (ABI): revised tolerance to pollution values for macroinvertebrate families and index performance evaluation. Revista de Biología Tropical 62: 294-273.

Roast S. D., Widdows J., Jones M. B. 2000. Disruption of swimming in the hyperbenthic mysid Neomysis integer (Peracarida: Mysidacea) by the organophosphate pesticide chlorpyrifos. Aquatic Toxicology 47 (3-4): 227-241.

Robinson C. T., Tocknet K., Ward J. V. 2002. The fauna of dynamic riverine landscapes. Freshwater Biology 47: 661-677.

Rodrigues Capítulo A., Tangorra M., Ocón C. 2001. Use of Benthic macroinvertebrate to assess the biological status of pampean strems in Argentina. Aquatic Ecology 35 (2):109-119.

Ronco A. E., Marino D. J. G., Abelando M., Almada P., Apartin C. D. 2016. Water quality of the main tributaries of the Paraná Basin: glyphosate and AMPA in surface water and bottom sediments. Environmental Monitoring and Assessment 188 (8): 458. 
Roy A. H., Rosemond A. D., Paul M. J., Leigh D. S., Wallace J. B. 2003. Stream macroinvertebrate response to catchment urbanisation (Georgia, U.S.A.). Freshwater Biology 48: 329-346.

Rubach M. N., Crum S. J. H., van der Brink P. J. 2011. Variability in the Dynamics of Mortality and Immobility Responses of Freshwater Arthropods Exposed to Chlorpyrifos. Archives of Environmental Contamination and Toxicology 60 (4): 708-721.

Saha S., Kaviraj A. 2008. Acute Toxicity of Synthetic Pyrethroid Cypermethrin to Some Freshwater Organisms. Bulletin of Environmental Contamination and Toxicology 80 (1): 49-52.

Sala J. M. 1973. Contribución al estudio geohidrológico del Noreste de la Provincia de Buenos Aires. Comité de Estudios de Aguas Subterráneas del Noreste (E.A.S.N.E.), Serie Técnica ${ }^{\circ}$ 24. Consejo Federal de Inversiones, Buenos Aires pp. 154.

Sala O. E., Chapin III F. S., Armesto J. J., Berlow E., Bloomfield J., Dirzo R., Huber-Sanwald E., Huenneke L. F., Jackson R. B., Kinzig A., Leemans R., Lodge D. M., Mooney H. A., Oesterheld M., Poff L., Sykes M. T., Walker B. H., Marilyn Walker M., Wall D. H. 2010. Global Biodiversity Scenarios for the Year 2100. Science 287 (5459): 1770-1774.

Sánchez M., Ferrando M. D., Andreu E. 2000. Physiological Perturbations in Several Generations of Daphnia magna Straus Exposed to Diazinon. Ecotoxicology and Environmental Safety 46: 87-94.

Sancho E., Banegas S., Villarroel M. J., Ferrando D. 2017. Impaired reproduction and individual growth of the water flea Daphnia magna as consequence of exposure to the non-ester pyrethroid etofenprox. Environmental Science and Pollution Research 25 (7): 6209-6217.

Sansiñena J. A., Peluso L., Salgado Costa C., Demetrio P. M., Mac Loughlin T. M., Marino D. J. G., Alcalde L., Natale G. S. 2018. Evaluation of the toxicity of the sediments from an agroecosystem to two native species, Hyalella curvispina (Crustacea: Amphipoda) and Boana pulchella (Amphibia: Anura), as potential environmental indicators. Ecological Indicators 93: 100-110.

Sarandón S. J., Flores C. C., Abbona E., lermanó M. J., Blandi M. L., Oyhamburu M., Presutti M. 2015. Análisis del uso de agroquímicos asociado a las actividades agropecuarias de la Provincia de Buenos Aires. En Relevamiento de la utilización de Agroquímicos en la Provincia de Buenos Aires - Mapa de Situación e incidencias sobre la salud. Defensoría del Pueblo de la Provincia de Buenos Aires, pp. 18-495.

Satapornvanit K., Baird D. J., Little D. C. 2009. Laboratory toxicity test and post-exposure feeding inhibition using the giant freshwater prawn Macrobrachium rosenbergii. Chemosphere 74 (9): 1209-1215.

Sathicq M. B., Arias M., Ferrando N. S., Benitez H. 2017. Optimización de un cultivo de Chlorella vulgaris en función del fotoperíodo y del control de pH. Biología Acuática 32 (Supl.): 146. 
Schäfer R. B., Caquet T., Siimes K., Mueller R., Lagadic L., Liess M., 2007. Effects of pesticides on community structure and ecosystem functions in agricultural streams of three biogeographical regions in Europe. Science of the Total Environment 382: 272-285.

Schäfer R. B., van der Brink P. J., Liess M. 2011. Impacts of pesticides on Freshwater Ecosystems. En Sánchez-Bayo F., van der Brink P. J., Mann R. M. (eds) Ecological Impacts of Toxic Chemicals. Bentham Science Publishers Ltd, USA, pp. 111-137.

Schäfer R. B., von der Ohe C. P., Rasmussen J., Kefford B. J., Beketov M. A., Schulz R., Liess M. 2012. Thresholds for the effects of pesticides on invertebrate communities and leaf breakdown in stream ecosystems. Environmental Science \& Technology 46: 5134-5142.

Schroer A. F. W., Belgers J. D. M., Brock T. C. M., Matser A. M., Maund S. J., Van den Brink P. J. 2004. Comparison of laboratory single species and field population-level effects of the pyrethroid insecticide $\lambda$-cyhalothrin on freshwater invertebrates. Archives of Environmental Contamination and Toxicology 46: 324-335.

Schulz R., Liess. M. 1999. A field study of the effects of agriculturally derived insecticide input on stream macroinvertebrate dynamics. Aquatic Toxicology 46: 155-176.

Schulz R. 2001. Rainfall-induced sediment and pesticide input from orchards into the Lourens River, Western Cape, South Africa: importance of a single event. Water Research 35 : 1869-1876.

Schulz R. 2004. Field studies on exposure, effects, and risk mitigation of aquatic nonpointsource insecticide pollution: a review. Journal of Environmental Quality 33: 419-448.

SenthilKumar P., Samyappan K., Jayakumar S., Deecaraman M. 2007. Impact of chlorpyrifos on the neurosecretory cells in a freshwater field crab, Spiralothelphusa hydrodroma. Research Journal of Agriculture and Biological Sciences 3 (6): 625-630.

Shen M-F., Kumar A., Ding S-Y., Grocke S. 2012. Comparative study on the toxicity of pyrethroids, a-cypermethrin and deltamethrin to Ceriodaphnia dubia. Ecotoxicology and Environmental Safety 78: 9-13.

Scheihing R., Labarca P. Cardenas L., Nespolo R. F. 2011 Viability selection on body size in a non-marine ostracod. Hydrobiologia 671:193-203.

Siefert R. E. 1987. Effects of Dursban (Chlorpyrifos) on aquatic organisms in enclosures in a natural pond. US EPA, Final Report.

Smith V. H., Tilman G. D., Nekola J. C. 1999. Eutrophication: impacts of excess nutrient inputs on freshwater, marine, and terrestrial ecosystems. Environmental Pollution 100: 179-196

Sokal R. R., Rohlf F. J. 1995. Biometry: The Principles and Practice of Statistics in Biological Research, 3rd edition. W.H. Freeman and Co, New York. pp. 887.

Solis M. 2016. Impacto de agroquímicos en arroyos de cuencas rurales. Tesis Doctoral. Facultad de Ciencias Naturales y Museo, Universidad Nacional de La Plata. 131pp. 
Solis M., Mugni H., Hunt L., Marrochi N., Fanelli S., Bonetto C. 2016. Land use effect on invertebrate assemblages in Pampasic streams (Buenos Aires, Argentina). Environmental Monitoring and Assessment 188: 539.

Solis M., Mugni H., Fanelli S., Bonetto C. 2017. Effect of agrochemicals on macroinvertebrate assemblages in Pampasic streams, Buenos Aires, Argentina. Environmental Earth Sciences 76: 180.

Solis M., Bonetto C., Marrochi N., Paracampo A., Mugni H. 2018. Aquatic macroinvertebrate assemblages are affected by insecticide applications on the Argentine Pampas. Ecotoxicology and Environmental Safety 148: 11-16.

Spears T., Abele L. G. 2000. Branchiopod monophyly and interordinal phylogeny inferred from 18 S ribosomal DNA. Journal of Crustacean Biology 20 (1): 1-24.

Stark J. D., E. Banks J. E. 2003. Population-level effects of pesticides and other toxicants on arthropods. Annual Review of Entomology 48: 505-519.

Stenderup J. T., Olesen J., Glenner H., 2006. Molecular phylogeny of the Branchiopoda (Crustacea) - Multiple approaches suggest a 'diplostracan' ancestry of the Notostraca. Molecular Phylogenetics and Evolution 41: 182-194.

Suren A. M. 1994. Macroinvertebrate communities of streams in western Nepal: effects of altitude and land use. Freshwater Biology 32: 323-336.

ter Braak C. J. F., Smilauer P. 1998. CANOCO Reference Manual and User's Guide to Canoco for Windows: Software for Canonical Community Ordination (ver. 4). Ithaca, NY: Microcomputer Power, pp. 351.

Thomas K. E., Lazor R., Chambers P. A., Yates A. G. 2018. Land-use practices influence nutrient concentrations of southwestern Ontario streams. Canadian Water Resources Journal, doi: 10.1080/07011784.2017.1411211

Tomlin C. D. S. 2000. The pesticide manual, a world compendium. Crop Protection Pubications, Farnham, Surrey, UK.

Tongbai W., Boonplueng R. Damrongphol P. 2012. Enzymatic responses of the riceland prawn, Macrobrachium lanchesteri, to chlorpyrifos exposure. Biologia 67 (4) Section Zoology: 762-766.

Toumi H., Boumaiza M., Millet M., Radetski C. M., Felten V., Fouque C., Férard J. F. 2013. Effects of deltamethrin (pyrethroid insecticide) on growth, reproduction, embryonic development and sex differentiation in two strains of Daphnia magna (Crustacea, Cladocera). Science of the Total Environment 458-460: 47-53.

Trautmann N., Carlsen W. S., Krasny M. E., Cunningham C. M. 2001. Assessing Toxic Risk. National Science Teachers Association Press, Arlington, Virginia. pp. 117. 
Tsui M. T. K., Chu L. M. 2004. Comparative toxicity of glyphosate-based herbicides: aqueous and sediment porewater exposures. Archives of Environmental Contamination and Toxicology 46: 316-323.

Urabe J. 1991. Effect of food concentration on growth, reproduction and survivorship of Bosmina longirostris (Cladocera). An experimental study. Freshwater Biology 25 (1): 1-8.

U. S. Environmental Protection Agency (USEPA) 1992. National Toxicology Program. Executive Summary of Safety and Toxicity Information: Chlorpyrifos. Chemical Evaluation Committee Draft Report. EPA 2921-88-2.

U. S. Environmental Protection Agency (USEPA) 1996. Effects Test Guidelines Gammarid Acute Toxicity Test. EPA 712-C-96-130.

U. S. Environmental Protection Agency (USEPA) 2000. Methods for Measuring the Toxicity and Bioaccumulation of Sediment-associated Contaminants with Freshwater Invertebrates, 2da edition. Washington, DC. EPA 600/R-99/064.

U. S. Environmental Protection Agency (USEPA) 2002. Methods for Measuring the Acute Toxicity of Effluents and Receiving Waters to Freshwater and Marine Organisms, 5th edition. Washington, DC. EPA-821-R-02-012

U. S. Environmental Protection Agency (USEPA) 2015. Species Sensivity Distribution Generator. Disponible en https://www.epa.gov/caddis-vol4/caddis-volume-4-dataanalysis-download-software\#tab-3 Último acceso: Septiembre 2018.

USGS 2015. Servicio Geológico de Estados Unidos, Earth Explorer. Disponible en http://earthexplorer.usgs.gov/ Último acceso: Diciembre 2015.

UTT 2018. Unión de los Trabajadores de la Tierra. Ley de Creación del Fondo Fiduciario Público de crédito para la Agricultura Familiar. Disponible en http://uniondetrabajadoresdelatierra.com.ar/2018/04/18/ley-creacion-del-fondo-fiduciariopublico-de-credito-para-la-agricultura-familiar-crepaf/ Último aceso: Agosto 2018.

van der Hoeven N. 1998. Power Analysis for the NOEC: What is the Probability of Detecting Small Toxic Effects on Three Different Species using the Appropriate Standardized Test Protocols? Ecotoxicology 7 (6): 355-361.

van Doorslaer W., Toks R. S., Jeppesen E., De Meester L. 2007. Adaptive microevolutionary responses to simulated global warming in Simocephalus vetulus: a mesocosm study. Global Change Biology 13: 878-886.

van Straalen N. 2002. Threshold models for species sensitivity distributions applied toaquatic risk assessment for zinc. Environmental Toxicology and Pharmacology 11: 167-172.

van Wijngaarden R., Leeuwangh P., Lucassen W. G., Romijn K., Ronday R., van Der Velde R., Willigenbur W. 1993. Acute toxicity of chlorpyrifos to fish, a newt, and aquatic invertebrates. Bulletin of Environmental Contamination and Toxicology 51(5): 716-723. 
Varela L. B., Laurencena P. C., Kruse E. E., Deluchi M., Rojo A. 2002. Reconocimiento de la relación aguas superficiales-aguas subterráneas en el arroyo del Gato, provincia de Buenos Aires. En Groundwater and Human Development. Bocanegra, E., Martínez, D, Massone, H. (Eds) pp. 1334-1341.

Verslycke T., Roast S. D., Widdows J., Jones M. B., Janssen C. R. 2004. Cellular energy allocation and scope for growth in the estuarine mysid Neomysis integer (Crustacea: Mysidacea) following chlorpyrifos exposure: a method comparison. Journal of Experimental Marine Biology and Ecology 306: 1-16.

Vijverberg H. P. M., van den Bercken J. 1990. Neurotoxicological effects and the mode of action of Pyrethroid insecticides. Critical Reviews in Toxicology 21(2): 105-126.

Villulla, J. M. 2006. Cambios sociales y degradación de la producción en el cinturón hortícola platense. Trabajo final de grado. Universidad Nacional de La Plata. Facultad de Humanidades y Ciencias de la Educación.

von Bertalanffy, L. 1938. A quantitative theory of organic growth. Human Biology 10 (2): 181213.

Walker P. D., Wijnhoven S., van der Velde G. 2013. Macrophyte presence and growth form influence macroinvertebrate community structure. Aquatic Botany 104: 80-87.

Wang S., Chen H. Y. H., Tan Y., Fan H., Ruan H. 2016. Fertilizer regime impacts on abundance and diversity of soil fauna across a poplar plantation chronosequence in coastal Eastern China. Scientific Reports 6: Article number: 20816.

Weber J., Halsall C. J., Muir D., Texeira C., Small J., Solomon K., Hermanson M., Hung H., Bidleman T. 2010. Endosulfan, a global pesticide: a review of its fate in the environment and occurrence in the Arctic. Science of the Total Environment 408: 2966-2984.

Weis J. S., Cristini A., Ranga Rao A. 1992. Effects of pollutants on molting and regeneration in Crustacea. American Zoologist 32 (3): 495-500

Wendt-Rasch L., Friberg-Jensen U., Woin P., Christoffersen K. 2003. Effects of the pyrethroid insecticide cypermethrin on a freshwater community studied under field conditions. II. Direct and indirect effects on the species composition. Aquatic Toxicology 63 (4): 373389.

Wetzel R. G. 1981. Limnología. 2da edición. Editorial Omega, Barcelona pp. 679.

Williner V., Collins P. A. 2003. Effects of Cypermethrin on the Freshwater Crab Trichodactylus borellianus (Crustacea: Decapoda: Braquiura). The Bulletin of Environmental Contamination and Toxicology 71: 106-113.

Willis K. J., Ling N., Chapman M.A. 1995. Effects of temperature and chemical formulation on the acute toxicity of pentachlorophenol to Simocephalus vetulus (Schoedler, 1858) (Crustacea: Cladocera). New Zealand Journal of Marine and Freshwater Research 29 (2): 289-294. 
Willis K. J., Ling N. 2004. Toxicity of the aquaculture pesticide cypermethrin to planktonic marine copepods. Aquaculture Research 35: 263-270.

Woods M., Kumar A., Correll R. 2002. Acute toxicity of mixtures of hlorpyrifos, profenofos, and endosulfan to Ceriodaphnia dubia. Bulletin of Environmental Contamination and Toxicology 68: 801-808.

Wu Y., Lin C., Yuan L., 2007. Characteristics of six cladocerans in relation to ecotoxicity testing. Ecological Indicators 7: 768-775.

You J., Weston D.P., Lydy M.J. 2004. A sonication extraction method for the analysis of pyrethroid, organophosphate, and organochlorine pesticides from sediment by gas chromatography with electron-capture detection. Archives of Environmental Contamination and Toxicology 47 (2): 141-147.

Young R. G., Huryn A. D. 1999. Effects of land use on stream metabolism and organic matter turnover. Ecological Applications 9 (4): 1359-1376.

Zalizniak L., Nugegoda D. 2006. Effect of sublethal concentrations of chlorpyrifos on three successive generations of Daphnia carinata. Ecotoxicology and Environmental Safety 64 (2): 207-214.

Zhang M., Muñoz-Mas R., Martínez-Capel F., Qu X., Zhang H., Peng W., Liu X. 2018. Determining the macroinvertebrate community indicators and relevant environmental predictors of the Hun-Tai River Basin (Northeast China): A study based on community patterning. Science of the Total Environment 634: 749-759.

Zhou Q., Zhang J., Fu J., Shi J., Jiang G. 2008. Biomonitoring: An appealing tool for assessment of metal pollution in the aquatic ecosystem. Analytica Chimica Acta 606 (2): 135-150.

Zou E., Fingerman M. 1997. Synthetic estrogenic agents do not interfere with sex differentiation but do inhibit molting of the cladoceran Daphnia magna. Bulletin of Environmental Contamination and Toxicology 58: 596-602. 